\title{
ESTUDIO DE LA RESPUESTAA ÓPTICA DE NANOESTRUCTURAS PLAMÓNICAS COMPLEJAS
}

\author{
Tesis de Doctorado
}

\section{LUIS JOAQUIN MENDOZA HERRERA}

Presentada ante la Facultad de Ingeniería de la Universidad Nacional de La Plata como requisito para la obtención del grado académico de

\section{DOCTOR EN INGENIERÍA}

Dirección de tesis:

Director: Daniel C. Schinca.

Codirector: Lucía B. Scaffardi

Jurado de Tesis:

Dra. María Laura Azcárate

Dr. Ricardo Depine

Dr. Marcelo Trivi

Fecha de la defensa oral y pública: 6 de Marzo de 2018 

Dedicado

a mis hijos

Helian y Ethan

y a mi adorable

compañera Dary. 

Un erudito cruzaba el río en una balsa y aprovechó la ocasión para entablar conversación con Nasrrudín...

¿Conoce usted la gramática?, a lo que Nasrrudín le contestó: No, en absoluto.

Permítame decirle, que ha perdido usted la mitad de su vida, le dijo el erudito con desdén.

Poco después, Nasrrudín pregunta al erudito: Sabe usted nadar. No, le contesta el erudito horrorizado.

Pues permítame que le diga que ha perdido usted el resto de su vida, porque nos estamos hundiendo.

Nasrrudín Hocca 


\section{Resumen}

En consonancia con los recientes desarrollos en nanociencia y nanotecnología, la óptica a escala nanométrica (Nanoóptica o Nanofotónica) está en el centro del interés científico y tecnológico a nivel mundial. Desde comienzos de la década de 1990, el número de trabajos relacionados con esta temática se ha incrementado casi exponencialmente. Una de las áreas de mayor actividad dentro de la Nanofotónica resulta del estudio de la interacción de la luz con superficies o estructuras metálicas con dimensiones típicas de decenas de nm. Dependiendo de las características del material, del medio y de la longitud de onda, se producen oscilaciones colectivas de los electrones libres del metal (plasmones) que generan absorbancias en determinadas bandas del espectro de luz incidente. Estas fluctuaciones de carga eléctrica son acompañadas por oscilaciones acopladas de campo electromagnético, por lo que las resonancias se llaman plasmones polaritones, y al área de investigación se la conoce como Plasmónica. Estos campos electromagnéticos resultan confinados en volúmenes nanométricos, por lo que resultan en refuerzos de campo de varios órdenes de magnitud. Una de las líneas frecuentemente analizadas en esta área se orienta al estudio de las propiedades plasmónicas de nanopartículas esféricas de metales nobles. Con el avance de diferentes métodos de síntesis, en particular la síntesis por ablación láser, es posible generar nanopartículas de diferentes metales, con diferentes capas de cobertura y hasta de formas no esféricas.

El desarrollo de esta tesis incluye aspectos teóricos y experimentales complementarios. La motivación principal de esta tesis en el aspecto teórico, apunta a extender el estudio de las propiedades plasmónicas de nanopartículas esféricas de 
metales no nobles, de estructura compuesta (núcleo y simple o doble cubierta) como así también de geometría no esférica. Dichas propiedades plasmónicas se analizan a través de la resonancia que presenta el espectro de extinción óptica de los coloides de nanopartículas fabricadas por ablación láser de femtosegundos o por síntesis química. Estos espectros pueden reproducirse utilizando teoría de Mie para partículas esféricas, que incluye, entre otros parámetros, la función dieléctrica del material de la partícula. Para tamaños por debajo de unas pocas decenas de nanómetros, esta última no sólo depende de la longitud de onda sino también del tamaño (radio).

Para describir la dependencia de la función dieléctrica, con la frecuencia y con el tamaño de la nanopartículas, primero se modeló la dependencia de la función dieléctrica de volumen con la longitud de onda como la suma de dos contribuciones: la contribución de los electrones libres y la contribución de los electrones ligados. Luego se incorporaron correcciones por tamaño a la función dieléctrica así calculada. Para realizar estas correcciones se requería conocer parámetros tales como la frecuencia de plasma y la constante de amortiguamiento de cada metal analizado. En muchos casos, no se conocían los valores de estos parámetros, haciéndose necesaria su determinación. En esta tesis se realizó un exhaustivo estudio del tema, lográndose determinar la frecuencia de plasma y la constante de amortiguamiento de 9 metales diferentes, desarrollando un novedoso método que utiliza como punto de partida las medidas experimentales de la función dieléctrica de volumen. El método desarrollado, que fue aplicado y validado para nanopartículas de metales nobles, permitió la determinación de aquellos parámetros con una mayor exactitud en un rango amplio de longitudes de onda y permitió extender el estudio de las propiedades plasmónicas a nanopartículas de metales de transición como son Ti, Ta, Fe, V, Pt y Al, entre otros.

Por otra parte, se abordó la descripción teórica de la extinción y la absorción de nanopartículas esféricas formadas por un núcleo y dos cubiertas desarrollando la teoría de Mie para ese tipo de estructura compleja considerando condiciones de contorno en las dos interfaces, incluyendo la función dieléctrica de cada material modificada por tamaño y calculando los refuerzos de campo en cada una de las regiones. 
En el caso de nanopartículas con características magnéticas, se describió teóricamente la dependencia de la permeabilidad magnética con la frecuencia utilizando la ecuación de Gilbert y utilizando la expresión determinada por Landau para nanopartículas esféricas de núcleo desnudo. Se calculó la contribución de las corrientes de Eddy a la extinción óptica para el caso de nanopartículas complejas de núcleo y dos cubiertas, destacándose la importancia de su inclusión.

Para nanoestructuras no esféricas, se implementó la aproximación de dipolos discretos (DDA) para calcular la eficiencia de extinción, previamente validada por comparación con espectros de extinción obtenidos de la teoría de Mie para nanopartículas esféricas. Esta aproximación fue utilizada para describir la respuesta de cristales fotónicos formados por arreglos de nanopartículas esféricas con características magnéticas.

En cuanto al abordaje de la parte experimental, se han fabricado nanopartículas en solución coloidal por ablación láser de femtosegundos, a partir de blancos sólidos de diferentes metales. A partir del análisis de los espectros de extinción se ha podido determinar la distribución de tamaños como así también la composición y estructura de las especies que componen los coloides mediante el ajuste por teoría de Mie, incluyendo las correcciones a la función dieléctrica por tamaño. Estos estudios han sido complementados por otras técnicas como microscopía AFM y TEM. También se ha aplicado el mismo tipo de análisis a la determinación de tamaño de nanopartículas en solución coloidal fabricadas por síntesis química. 


\section{Índice General}

Resumen I I

Índice de Figuras $\quad$ VII

Índice de Tablas $\quad$ XIV

$\begin{array}{ll}\text { 1. Introducción } & 1\end{array}$

\begin{tabular}{ll}
\hline 2. Extinción, Scattering y absorción & 7
\end{tabular}

2.1. Modelado de la función dieléctrica . . . . . . . . . . . . . . . . . . . . . 8

2.1.1. Determinación de $\omega_{p} \mathrm{y} \gamma$. . . . . . . . . . . . 11

2.1.2. Corrección de la función dieléctrica por tamaño . . . . . . . . . 20

2.2. Permeabilidad magnética . . . . . . . . . . . . . . . . . 25

2.3. Extinción de nanopartículas sin cubierta . . . . . . . . . . . . . . . . 29

2.4. Extinción de nanopartículas con una cubierta . . . . . . . . . . . . . . 30

3. Fabricación de nanopartículas por ablación láser $\quad 37$

4. Caracterización de nanopartículas por espectroscopía de extinción $\begin{array}{ll}\text { óptica } & 47\end{array}$

4.1. Caracterización de nanopartículas de Au. . . . . . . . . . . . . . . . . . 51

4.2. Caracterización de nanopartículas de magnetita. . . . . . . . . . . . . . 53

4.3. Caracterización de nanopartículas núcleo-cubierta de $\mathrm{Fe}_{3} \mathrm{O}_{4}$-Au. . . . . 56 
4.4. Caracterización de nanopartículas de Al fabricadas por ablación láser . 59

4.5. Caracterización de nanopartículas no esféricas . . . . . . . . . . . . . . 64

4.5.1. Aproximación de dipolos discretos . . . . . . . . . . . . . . . . 64

4.5.2. Caracterización de nanopartículas no esféricas de Na . . . . . . 70

\begin{tabular}{|l|l|}
\hline 5. Refuerzo de campo en nanopartículas esféricas & 75
\end{tabular}

5.1. Teoría de Mie vs aproximación dipolar . . . . . . . . . . . . . . . . 76

5.1.1. Influencia del metal del núcleo . . . . . . . . . . . . . . . . . . . 82

5.1.2. Modelo de Hibridación . . . . . . . . . . . . . . . . . . . . . . . 83

5.2. Refuerzo de campo en estructuras esféricas con doble cubierta . . . . . 86

5.3. Extinción óptica de un arreglo lineal de nanopartículas . . . . . . . . . 96

$\begin{array}{ll}\text { 6. Conclusiones Generales } & 113\end{array}$

\begin{tabular}{ll}
\hline Producido de la tesis & 117
\end{tabular}

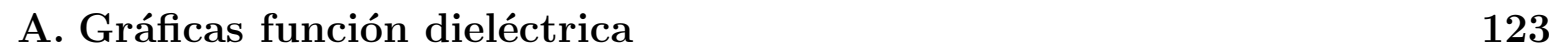

A.1. Otras funciones dieléctricas utilizadas . . . . . . . . . . . . . . . . . . . 123

A.2. Gráficas de la función dieléctrica . . . . . . . . . . . . . . . . . . . . . . 124

B. Extinción de nanopartículas esféricas 135

B.1. Ecuación de Ondas . . . . . . . . . . . . . . . . . . . . . . 135

B.2. Campos en nanopartículas esféricas con dos cubiertas . . . . . . . . . . 138

B.3. Nanopartículas esféricas con una cubierta . . . . . . . . . . . . . . . . . 143

B.4. Nanopartículas esféricas sin cubierta . . . . . . . . . . . . . . . 144

B.5. Cálculo de los coeficientes de Mie . . . . . . . . . . . . . . . . . . . . . 144

B.6. Aproximación de Mie para nanopartículas pequeñas . . . . . . . . . . . 146

B.7. Extinción de nanopartículas magnéticas . . . . . . . . . . . . . . . . . . 149

$\begin{array}{ll}\text { C. Aproximación de Dipolos Discretos (DDA) } & 157\end{array}$

\begin{tabular}{ll}
\hline D. Hibridación & 161
\end{tabular}

D.1. Plasmones en esferas metálicas . . . . . . . . . . . . . . . . . . . . . . . 162 
D.2. Plasmones en cavidades esféricas . . . . . . . . . . . . . . . . 167

D.3. Plasmones en nano-cubiertas metálicas . . . . . . . . . . . . . . . . . . 167

\begin{tabular}{|l|l}
\hline Agradecimientos & 195
\end{tabular} 


\section{Índice de Figuras}

1.1. Nanopartícula núcleo-cubierta (core-shell). . . . . . . . . . . . . . . . . 3

2.1. Gráficas de la regresión lineal utilizada para determinar $\omega_{p}^{2}$ y $\gamma$ para el Au. 13

2.2. Partes real e imaginaria de la contribución de los electrones libres a la función dieléctrica experimental del Au. . . . . . . . . . . . . . . . . . 15

2.3. $\quad$ Partes real e imaginaria de la contribución de los electrones libres a la función dieléctrica del Al . . . . . . . . . . . . . . . . . . . . . . . . . . 17

2.4. Partes real (línea continua) e imaginaria (línea segmentada) de las contribuciones de los electrones libres y ligados a la función dieléctrica del Al. . . . . . . . . . . . . . . . . . . . . . . 19

2.5. Partes real e imaginaria de la contribución de los electrones libres a la función dieléctrica de $\mathrm{Au}$. . . . . . . . . . . . . . . . . . . . . . . . . 23

2.6. Partes imaginaria y real de la función dieléctrica de volumen más las correcciones de las contribuciones de los electrones libres y ligados. . . . 24

2.7. Partes real e imaginaria de la permeabilidad magnética efectiva de un compuesto de magnetita . . . . . . . . . . . . . . . . . . 28

2.8. Comparación entre los valores experimentales para wt=8\% [19] y valores teóricos con $\alpha_{G}=0.32$ de la permeabilidad magnética efectiva de magnetita. 29

2.9. Extinción de NPs con núcleo de $\mathrm{Al}$ y $\mathrm{Au}$, para diferentes radios de las NPs 30

2.10. Extinción de NPs con núcleo de $\mathrm{Al}$ y cubierta de $\mathrm{Al}_{2} \mathrm{O}_{3}$ para diferentes espesores de la cubierta. . . . . . . . . . . . . . . . . . . . . . 31 
2.11. Espectros de Extinción de NPs con núcleo de $\mathrm{Al}_{2} \mathrm{O}_{3}$ y cubierta de $\mathrm{Al}$ para diferentes espesores de la cubierta. . . . . . . . . . . . . . . . 32

2.12. Evolución de la oxidación de NPs de Al para diferentes radios de la NP. 33

2.13. Espectros de extinción para NPs esféricas de magnetita con una cubierta de Au. . . . . . . . . . . . . . . . . . . 34

3.1. Esquema de amplificación de pulsos utilizando CPA y fabricación de NPs por ablación láser. . . . . . . . . . . . . . . . . . . . . . . . . . 39

3.2. Resumen del proceso de ablación con un láser de fs (no térmica) . . . . 41

3.3. Ablación de un láser de femtosegundos sobre un blanco sumergido. . . . 42

3.4. Fotografias de la extracción de material para un blanco de $\mathrm{Al}$ utilizando un láser de $\mathrm{fs} . \ldots \ldots \ldots$. . . . . . . . . . . . . . . 43

3.5. Umbral de ablación para el Al. . . . . . . . . . . . . . . . . 44

4.1. Esquema de los procesos de scattering y extinción de un conjunto de partículas. . . . . . . . . . . . . . . . . . . . . 49

4.2. Ajuste del espectro experimental de NPs de Au. . . . . . . . . . 52

4.3. Espectro experimental y teórico de NPs de magnetita en agua. . . . . . 54

4.4. Espectro experimental y teórico de NPs de magnetita en n-heptano. . . 55

4.5. Longitud de onda y ancho a la altura $70 \%$ del pico plasmónico de magnetita. . . . . . . . . . . . . . . . . . 57

4.6. Curvas de nivel del pico y ancho del pico plasmónico para los espectros experimentales de NPs de $\mathrm{Fe}_{3} \mathrm{O}_{4}$-Au dadas en Lingyan et al. . . . . . . 58

4.7. Espectro experimental de NPs de Al fabricadas por ablación láser en n-heptano, con su respectivo ajuste y distribución de tamaños. . . . . . 59

4.8. Espectro experimental de NPs de Al fabricadas por ablación láser en agua, con su respectivo ajuste y distribución de tamaños. . . . . . . . . 60

4.9. Fotos AFM de NPs de Al fabricadas por ablación láser en agua y perfiles de algunas líneas seleccionadas. . . . . . . . . . . . . . . . . 62 
4.10. Radios de las diferentes especies de NPs obtenidas utilizando la ablación láser sobre un blanco de $\mathrm{Al}$ en agua, en función de la energía de ablación. 63

4.11. Espectro de extinción de NPs de Au utilizando la teoría de Mie y la DDA 65

4.12. Evolución del ancho y ubicación del pico plasmónico para NPs de Au utilizando la teoría de Mie y la DDA. . . . . . . . . . . . . . . . 66

4.13. Espectro de extinción de NPs cúbicas de Au utilizando la DDA. . . . . 67

4.14. Ubicación del pico plasmónico para NPs de $\mathrm{Au}$ con núcleo de $\mathrm{SiO}_{2}$ esféricas y cubicas. . . . . . . . . . . . . . . . . . 67

4.15. Espectro de extinción de NPs de sodio de diferente geometría y sus respectivas discretizaciones para un radio efectivo de $2.5 \mathrm{~nm}$. . . . . . . 68

4.16. Espectro de extinción de NPs en forma de decaedro de Na. . . . . . . . 69

4.17. Ubicación y ancho del pico plasmónico de Nps de Na en forma de octaedro truncado. . . . . . . . . . . . . . . . . 70

4.18. Curvas de nivel para Nps de Na con la ubicación y el ancho del pico plasmónico del espectro experimental. . . . . . . . . . . . . . . . . . . . 71

4.19. Espectros experimental y ajuste teórico de Nps de Na. . . . . . . . . . 72

5.1. Espectros de extinción de NPs esféricas de $\mathrm{Au} \mathrm{y} \mathrm{Al.} \mathrm{.} \mathrm{.} \mathrm{.} \mathrm{.} \mathrm{.} \mathrm{.} \mathrm{.} \mathrm{.} \mathrm{.} \mathrm{.} \mathrm{.} 78$

5.2. Comparación entre la teoría de Mie y la aproximación dipolar para NPs:

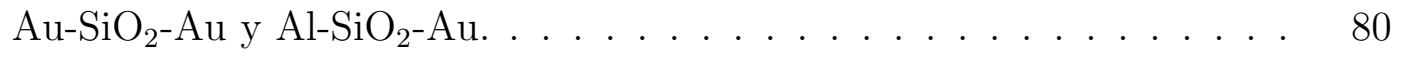

5.3. Influencia de la corrección de la función dieléctrica en los espectros de

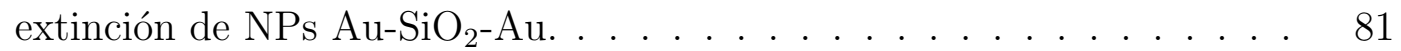

5.4. Espectros de extinción de NPs de metal- $\mathrm{SiO}_{2}-\mathrm{Au}$, para núcleos de $\mathrm{Au}$, $\mathrm{Ag}, \mathrm{Cu} \mathrm{y} \mathrm{Al.} \mathrm{.} \mathrm{.} \mathrm{.} \mathrm{.} \mathrm{.} \mathrm{.} \mathrm{.} \mathrm{.} \mathrm{.} \mathrm{.} \mathrm{.} \mathrm{.} \mathrm{.} \mathrm{.} \mathrm{.} \mathrm{.} \mathrm{.} \mathrm{.} \mathrm{.} \mathrm{.} \mathrm{.} \mathrm{.} 82$

5.5. Condiciones de resonancia, hibridación y espectros de extinción de nanopartículas esféricas de $\mathrm{Au}-\mathrm{SiO}_{2}$-Au. . . . . . . . . . . . . . . 85

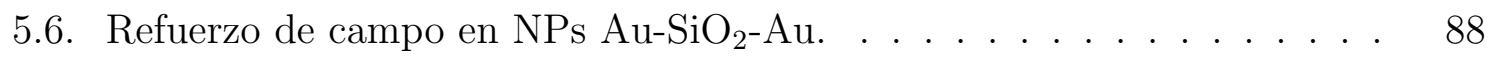

5.7. Refuerzo de campo en ${\mathrm{NPs} \mathrm{Ag}-\mathrm{SiO}_{2}-\mathrm{Au} .}^{2}$. . . . . . . . . . . . . . . . 89

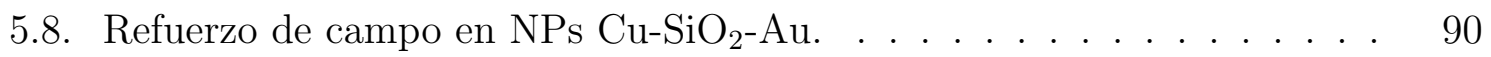

5.9. Refuerzo de campo en $\mathrm{NPs}_{\mathrm{Al}-\mathrm{SiO}_{2}-\mathrm{Au} .}$. . . . . . . . . . . . . . . . . . 91 
5.10. Valores máximos del refuerzo de campo en el exterior de NPs con la configuración metal-SiO $\mathrm{S}_{2} \mathrm{Au}$. . . . . . . . . . . . . . . . . . . 92

5.11. Valores máximos del refuerzo de campo en la capa de $\mathrm{SiO}_{2}$ en $\mathrm{NPs}$ con la configuración metal- $\mathrm{SiO}_{2}$-Au. . . . . . . . . . . . . . . . . . . . . 93

5.12. Valores máximos del refuerzo de campo en el exterior de NPs con la configuración metal-SiO $\mathrm{S}_{2} \mathrm{Au}$.

5.13. Valores máximos del refuerzo de campo en el interior de NPs con la configuración metal- $\mathrm{SiO}_{2}-\mathrm{Au}$.

5.14. Esquema correspondiente a un arreglo líneal de NPs pequeñas de diámetro $2 R$, separadas una distancia $D$ entre ellas. . . . . . . . . . . 96

5.15. Espectro de extinción para un arreglo líneal de NPs esféricas de Ag. . . 97

5.16. Espectro de extinción para un arreglo líneal de NPs esféricas de Ag. . . 98

5.17. Espectros de extinción de NPs de $\mathrm{Fe}_{3} \mathrm{O}_{4}-\mathrm{SiO}_{2}$. . . . . . . . . . . . . . . 100

5.18. Partes real e imaginarias de la polarizabilidad dipolar y efectiva en NPs de $\mathrm{Fe}_{3} \mathrm{O}_{4}-\mathrm{SiO}_{2}$. . . . . . . . . . . . . . . . . . . . . . . . . . . . . 101

5.19. Extinción para los modos TE y TM en un arreglo de $\mathrm{NPs}_{\mathrm{s}} \mathrm{Fe}_{3} \mathrm{O}_{4}-\mathrm{SiO}_{2} .102$

5.20. Influencia en la extinción óptica del número de NPs. . . . . . . . . . . . 103

5.21. Extinción para el modo TE en un arreglo de $\mathrm{NPs}$ de $\mathrm{Fe}_{3} \mathrm{O}_{4}-\mathrm{SiO}_{2}$. . . . . 104

5.22. Influencia del material de la cubierta en un arreglo de NPs con núcleo

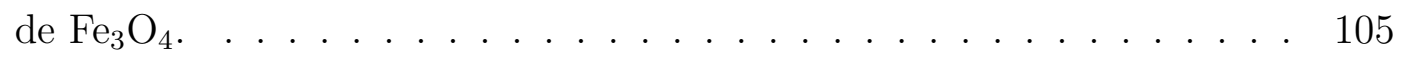

5.23. Influencia de la separación de las NPs en un arreglo de NPs con núcleo de $\mathrm{Fe}_{3} \mathrm{O}_{4} . \ldots \ldots \ldots \ldots$

5.24. Influencia del gradiente de campo en un arreglo de NPs con núcleo de $\mathrm{Fe}_{3} \mathrm{O}_{4}$. . . . . . . . . . . . . . . . . . 107

5.25. Influencia de la dispersión de tamaños en un arreglo de NPs con núcleo

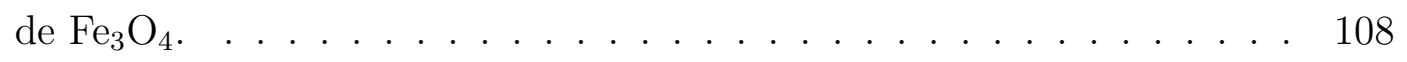

5.26. Ajuste de valores experimentales sin distribución de tamaños en las NPs y sin una variación del gradiente de campo magnético. . . . . . . . . . 109 
5.27. Ajuste de valores experimentales con una distribución de tamaños en las NPs correspondiente a $\sigma=0.25$. . . . . . . . . . . . . . . . . . . . . . 109

5.28. Ajuste de valores experimentales con una distribución de tamaños en las NPs correspondiente a $\sigma=0.25$ y un gradiente espacial de campo magnético equivalente a $\Delta d=11 \mathrm{~nm}$. . . . . . . . . . . . . . . . . . . . 110

A.1. Gráficas de la regresión lineal utilizada para determinar $\omega_{p}^{2}$ y $\gamma$ para la Ag. 125 A.2. Gráficas de la regresión lineal utilizada para determinar $\omega_{p}^{2}$ y $\gamma$ para el Cu. 126 A.3. Gráficas de la regresión lineal utilizada para determinar $\omega_{p}^{2}$ y $\gamma$ para el Al.127

A.4. Partes real e imaginaria de la contribución de los electrones libres a la función dieléctrica $\mathrm{Ag}$. . . . . . . . . . . . . . . . . . . . . . . . . . . . 128

A.5. Partes real e imaginaria de la contribución de los electrones libres a la función dieléctrica $\mathrm{Cu}$. . . . . . . . . . . . . . . . . . . . . . . . . . . . 129

A.6. Partes real e imaginaria de la contribución de los electrones libres a la función dieléctrica Al . . . . . . . . . . . . . . . . . . . . . . . . . . . . 130

A.7. Partes real e imaginaria de la contribución de los electrones libres a la

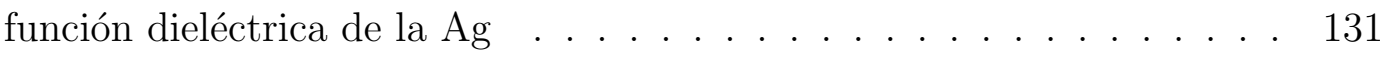

A.8. Partes real e imaginaria de la contribución de los electrones libres a la función dieléctrica del Cu. . . . . . . . . . . . . . . . . . . . . . . . . . 132

A.9. Partes real e imaginaria de la contribución de los electrones libres a la función dieléctrica del Al. . . . . . . . . . . . . . . . . . . . . . . . . . 133

B.1. Gráfico de las funciones esféricas de Bessel $j_{n}(k r)$ y $y_{n}(k r)$. . . . . . . 137

B.2. Configuración geométrica para una partícula esférica con núcleo y doble cubierta. . . . . . . . . . . . . . . . . . . 139

B.3. Gráfica de la función $\psi_{n}(\rho)=\rho j_{n}(\rho)$. . . . . . . . . . . . . . . . . . . 142

B.4. Gráfica de la función $\chi_{n}(\rho)=-\rho y_{n}(\rho)$. . . . . . . . . . . . . . . 142

B.5. Configuración geométrica de una nanopartícula núcleo-cubierta o core shell 144

B.6. Expresiones de la polarizabilidad para distintas configuiraciones geométricas y expresiones y estructuras. . . . . . . . . . . . . . . . . . 148 
B.7. Determinación del radio para el que la nanopartícula se encuentra en la aproximación dipolar. . . . . . . . . . . . . . . . . . . . . . . . . . . . . 148

B.8. Comparación entre diferentes aproximaciones para el cálculo de la extinción de NPs de diferentes tamaños. . . . . . . . . . . . . . . . . . 152

B.9. Gráfica de la parte imaginaria de la polarizabilidad de NPs en la configuración $\mathrm{Fe}_{3} \mathrm{O}_{4}-\mathrm{SiO}_{2}-\mathrm{Au}$. . . . . . . . . . . . . . . . . . . . . 155

C.1. Geometría de una NE compuesta por $N$ dipolos. . . . . . . . . . . . . . 158

D.1. Espectros de extinción de nanopartículas de $\mathrm{Au}$ en $\mathrm{SiO}_{2}$, utilizando la aproximación dipolar. . . . . . . . . . . . . . . . . . . . . . . . 166

D.2. Frecuencias de resonancia de cavidades, esferas y nanocubiertas de $\mathrm{Au}$, $\mathrm{Ag}, \mathrm{Al} \mathrm{e} \mathrm{Fe}$. . . . . . . . . . . . . . . . . . . . . . . . . . . . . 169

D.3. Frecuencias de resonancia de nanocubiertas de $\mathrm{Au}, \mathrm{Ag}, \mathrm{Al}$ e Fe para diferentes relaciones $x=R_{1} / R_{2} \ldots \ldots \ldots$. . . . . . . . . . . 170

D.4. Extinción de nanocubiertas de Au con núcleo y medio circundante de $\mathrm{SiO}_{2} .171$ 


\section{Índice de Tablas}

2.1. Valores $\omega_{p}$ y $\gamma$ determinados en este trabajo para $\mathrm{Au}, \mathrm{Ag}, \mathrm{Cu}$ y Al. 1ra columna: Metal (Función dieléctrica macroscópica experimental) . . . 14

2.2. Parámetros que describen la contribución de los electrones ligados a la función dieléctrica del Au y del Cu, utilizando la aproximación RPA. 18

2.3. Parámetros que describen la contribución de los electrones ligados a la función dieléctrica del Al. . . . . . . . . . . . . . . . . . . . . . . . . . 19

4.1. Especies de NPs presentes en la solución coloidal de Al en n-heptano y agua fabricadas por ablación láser . . . . . . . . . . . . . . 61

4.2. Resumen de la distribución de tamaños de NPs fabricadas por ablación láser sobre un blanco de $\mathrm{Al}$ en agua, para distintas energías de ablación 63

B.1. Resumen de la extinción óptica para diferentes rangos de longitud de onda y diferentes tamaños de NPs . . . . . . . . . . . . . . . . . . 153 
XVI 


\section{Capítulo 1}

\section{Introducción}

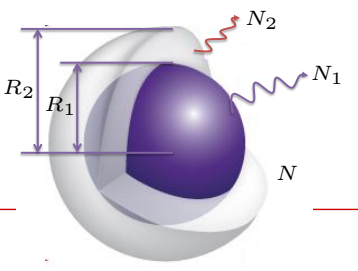

No hay que apagar la luz del otro para que brille la nuestra

Gandhi.

El estudio del scattering y absorción de radiación electromagnética por partículas pequeñas reconoce su primer trabajo fundamental en la publicación de Gustav Mie [1] en 1908 respecto del scattering de luz por partículas esféricas.

Durante los últimos años ha adquirido importancia creciente, el estudio de la interacción radiación visible-materia en una escala mucho menor que la longitud de onda de la luz, en la que ésta queda confinada a volúmenes nanoscópicos. En esta región de unas pocas decenas de nanómetros, la luz posee características especiales que han dado lugar a un cuerpo de conocimiento llamado Nanofotónica [2]. El conocimiento de las propiedades de la luz en volúmenes nanométricos constituye la base de diversas aplicaciones en nanoscopías ultrasensibles basadas en refuerzo de campo, espectroscopia micro Raman ultrasensible, etc. [3]. Un capítulo de esta nueva área es el estudio de propiedades ópticas de metales nanoestructurados conocida como Plasmónica [4, 5], que se entiende como la ciencia y la tecnología de la óptica basada en propiedades metálicas en la nanoescala.

Si bien las primeras observaciones experimentales de efectos ópticos en metales [6,7] son anteriores al trabajo de Mie, recién se comenzaron a explorar y entender a mediados del siglo XX [8, 9] introduciendo el concepto de oscilaciones colectivas de electrones libres, llamadas "plasmones superficiales". Kreibig y Zacharias [10] describieron las propiedades ópticas y electrónicas de nanopartículas (Nps) de oro y plata basados, por 
primera vez, en este concepto.

Varios desafíos tecnológicos actuales pueden ser superados utilizando las propiedades de los plasmones superficiales. Los estudios de las últimas décadas han permitido el desarrollo de elementos y técnicas ópticas basadas en plasmones tales como guías de onda pasivas, interruptores activos, máscaras litográficas, biosensores, etc.

Para determinar las eficiencias de scattering y absorción de una NP en función de la intensidad de la OEM incidente, se deben calcular los campos eléctricos y magnéticos en cada una de las regiones que conforman la NP haciendo uso de las ecuaciones de Maxwell.

La tasa de transferencia de energía electromagnética en todos los puntos del espacio, se define a partir del vector de Poynting [11, 12

$$
\mathbf{S}=\mathbf{E} \times \mathbf{H}
$$

donde $\mathbf{E}$ y $\mathbf{H}$ son los campos eléctrico y magnético en cada una de las regiones de la NP. Utilizando la definición del vector de Poynting se puede calcular la tasa neta $W$ de energía electromagnética que cruza una superficie cerrada $A$ que encierra un volumen $V$ 11, 12.

$$
W=-\int_{A} \mathbf{S} \cdot \mathbf{d} \mathbf{A}
$$

A partir de las tasas netas de scattering $W_{\text {sca }}$ y absorción $W_{a b s}$, se describen las secciones transversales de scattering $C_{s c a}=W_{s c a} / I_{i}$ y absorción $C_{a b s}=W_{a b s} / I_{i}$, que tienen unidades de área, donde $I_{i}$ es la intensidad de la onda incidente. Si la NP sobre la cual incide la onda es esférica de radio $R$, las eficiencias de scattering y absorción se definen como $Q_{s c a}=C_{s c a} /\left(\pi R^{2}\right)$ y $Q_{a b s}=C_{a b s} /\left(\pi R^{2}\right)$.

Para el caso particular de NPs con estructura núcleo-cubierta, como se indica en la Figura 1.1. es posible calcular las eficiencias de scattering y extinción utilizando las definiciones de los campos eléctricos y magnéticos en cada una de las regiones, como fue deducido por Gustav Mie $\mathbf{1 3}-\mathbf{1 7}$ : 


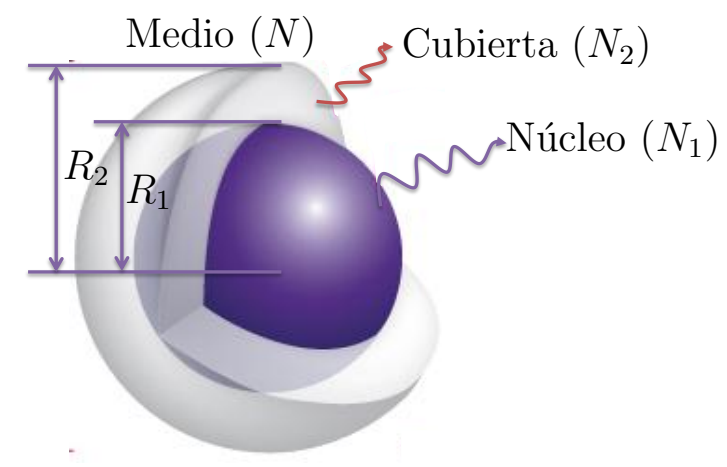

Figura 1.1: Nanopartícula núcleo-cubierta (core-shell) inmersa en un medio de indice $N$, de núcleo con radio $R_{1}$ y cubierta $R_{2}$ con indices $N_{1}$ y $N_{2}$ respectivamente

$$
\begin{aligned}
Q_{\text {scat }} & =\frac{2}{k^{2} R_{2}^{2}} \sum_{n=1}^{\infty}(2 n+1)\left(\left|a_{n}\right|^{2}+\left|b_{n}\right|^{2}\right) \\
Q_{\text {ext }} & =\frac{2}{k^{2} R_{2}^{2}} \sum_{n=1}^{\infty}(2 n+1) \operatorname{Re}\left(a_{n}+b_{n}\right)
\end{aligned}
$$

donde $R_{2}$ es el radio exterior de la NP. Las expresiones para la determinación de los coeficientes de la serie, $a_{n}$ y $b_{n}$ son:

$$
\begin{aligned}
a_{n} & =\frac{\mu m_{2} \psi_{n}^{\prime}(y)\left[\psi_{n}\left(m_{2} y\right)-A_{n} \chi_{n}\left(m_{2} y\right)\right]-\mu_{2} \psi_{n}(y)\left[\psi_{n}^{\prime}\left(m_{2} y\right)-A_{n} \chi_{n}^{\prime}\left(m_{2} y\right)\right]}{\mu m_{2} \xi_{n}^{\prime}(y)\left[\psi_{n}\left(m_{2} y\right)-A_{n} \chi_{n}\left(m_{2} y\right)\right]-\mu_{2} \xi_{n}(y)\left[\psi_{n}^{\prime}\left(m_{2} y\right)-A_{n} \chi_{n}^{\prime}\left(m_{2} y\right)\right]} \\
b_{n} & =\frac{\mu m_{2} \psi_{n}(y)\left[\psi_{n}^{\prime}\left(m_{2} y\right)-B_{n} \chi_{n}^{\prime}\left(m_{2} y\right)\right]-\mu_{2} \psi_{n}^{\prime}(y)\left[\psi_{n}\left(m_{2} y\right)-B_{n} \chi_{n}\left(m_{2} y\right)\right]}{\mu m_{2} \xi_{n}(y)\left[\psi_{n}^{\prime}\left(m_{2} y\right)-B_{n} \chi_{n}^{\prime}\left(m_{2} y\right)\right]-\mu_{2} \xi_{n}^{\prime}(y)\left[\psi_{n}\left(m_{2} y\right)-B_{n} \chi_{n}\left(m_{2} y\right)\right]}
\end{aligned}
$$

con

$$
\begin{aligned}
A_{n} & =\frac{\mu_{1} m_{2} \psi_{n}\left(m_{2} x\right) \psi_{n}^{\prime}\left(m_{1} x\right)-\mu_{2} m_{1} \psi_{n}^{\prime}\left(m_{2} x\right) \psi_{n}\left(m_{1} x\right)}{\mu_{1} m_{2} \chi_{n}\left(m_{2} x\right) \psi_{n}^{\prime}\left(m_{1} x-\mu_{2} m_{1} \chi_{n}^{\prime}\left(m_{2} x\right) \psi_{n}\left(m_{1} x\right)\right.} \\
B_{n} & =\frac{\mu_{1} m_{2} \psi_{n}^{\prime}\left(m_{2} x\right) \psi_{n}\left(m_{1} x\right)-\mu_{2} m_{1} \psi_{n}\left(m_{2} y\right) \psi_{n}^{\prime}\left(m_{1} x\right)}{\mu_{1} m_{2} \chi_{n}^{\prime}\left(m_{2} x\right) \psi_{n}\left(m_{1} x\right)-\mu_{2} m_{1} \chi_{n}\left(m_{2} x\right) \psi_{n}^{\prime}\left(m_{1} x\right)}
\end{aligned}
$$

donde $m_{1}=k_{1} / k=N_{1} / N, m_{2}=k_{2} / k=N_{2} / N, k_{i}=2 \pi N_{i} / \lambda, x=k_{1} R_{1}$ e $y=k_{2} R_{2}$. Las 
funciones $\psi_{n}(\rho)=\rho j_{n}(\rho), \chi_{n}(\rho)=-\rho y_{n}(\rho)$ y $\xi_{n}(\rho)=\rho h_{n}^{(1)}(\rho)$ dependen de las funciones de Bessel esféricas $y_{n}(\rho) y_{n}(\rho)$ y $h_{n}^{(1)}(\rho)$. En este caso, estos coeficientes dependen de los radios del núcleo $\left(R_{1}\right)$ y de la cubierta $\left(R_{2}\right)$, de la longitud de onda de la onda electromagnética (OEM) incidente y de los índices de refracción del núcleo $\left(N_{1}\right)$, de la cubierta $\left(N_{2}\right)$ y del medio circundante $(N)$, como también de las permeabilidades magnéticas relativas del núcleo $\left(\mu_{1}\right)$ y de la cubierta $\left(\mu_{2}\right)$. Para el caso particular de una NP simple se cumple que $N_{1}=N_{2}$ y $\mu_{1}=\mu_{2}$, condición que hace que los coeficientes $A_{n}$ y $B_{n}$ son ceros.

Como acabamos de mostrar, uno de los parámetros de vital importancia en la caracterización y descripción de las propiedades ópticas de nanoestructuras (NEs), es el índice de refracción de cada uno de los medios con los cuales interactúa la luz. En términos generales este indice de refracción $N$ es un complejo que presenta dependencia con la frecuencia $(\omega)$ a través de su relación con la permitividad dieléctrica relativa o función dieléctrica $\varepsilon(\omega)$ y de la permeabilidad magnética relativa:

$$
N=\sqrt{\varepsilon(\omega) \mu(\omega)}=n(\omega)+i \kappa(\omega)
$$

donde $n(\omega)$ y $\kappa(\omega)$ representan sus partes real e imaginaria. Una característica importante es que al disminuir el tamaño de la NP, la función dieléctrica presenta una fuerte dependencia con el tamaño de la NE.

Para los materiales que no tienen propiedades magnéticas el valor de $\mu(\omega)$ es la unidad. Cuando las NPs presentan propiedades magnéticas, debe conocerse previamente la dependencia de la permeabilidad con la frecuencia. Como se verá más adelante, este tema se abordará utilizando la ecuación de Gilbert.

El estudio de NEs metálicas de diversa complejidad es un tema que ha concitado gran interés en los últimos años. Varios autores [18-20] desarrollan estudios espectroscópicos de plasmones superficiales de Nps de algunas decenas de nanómetros de diámetro, analizando las características ópticas en función del tamaño para nanocilindros, nanoesferas tipo núcleo-cubierta y otras formas arbitrarias. Estas últimas ya no pueden ser descriptas utilizando la teoría de Mie, generando la necesidad de recurrir a métodos 
numéricos para estudiar los campos en cada una de las regiones que las conforman y poder analizar sus características plasmónicas. Existen varios métodos para este tipo de tratamientos, donde el más utilizado es el método de discretización por dipolos (DDA por sus siglas en ingles Discrete Dipole Approximation). Esta estrategia será utilizada, como se verá más adelante, para dos casos que presentan una geometría no esférica y/o disposición en arreglos tipo cristalino.

Este trabajo de tesis está orientado al estudio de la respuesta óptica de NEs simples, multicapas y de geometría arbitraria, respuesta que se utiliza en la caracterización de las NEs y sus propiedades plasmónicas. Algunas de estas NEs han sido fabricadas durante esta tesis por ablación láser de pulsos ultracortos o por síntesis química.

La tesis está dividida en 5 capítulos. Luego de la Introducción, el Capítulo 2 contiene la formulación teórica necesaria para interpretar y modelar los resultados experimentales de espectros de extinción óptica de NPs esféricas generadas por los métodos antes mencionados. Este capítulo incluye el estudio de la dependencia del índice de refracción con la frecuencia y con el radio de las NPs, para lo cual es necesario conocer los parámetros de Drude $\omega_{p}$ (frecuencia de plasma), $\gamma$ (constante de amortiguamiento de los electrones libres) y el coeficiente de Gilbert $\left(\alpha_{G}\right)$. Los parámetros de Drude fueron determinados con un novedoso método desarrollado en esta tesis y utilizado para metales nobles y de transición. Para el caso de NPs magnéticas (por ej. $\mathrm{Fe}_{3} \mathrm{O}_{4}$ ), fue posible idear un método también original para determinar el coeficiente de Gilbert que luego fue utilizado para calcular la permeabilidad magnética.

El Capítulo 3 está dedicado a describir el arreglo experimental utilizado para generar las muestras de NPs por ablación láser de femtosegundos en medios líquidos, como también el modelo standard que describe el proceso de fabricación.

El Capítulo 4 está dedicado a mostrar la caracterización de tamaño, estructura y configuración de los coloides antes generados a través del ajuste de los espectros experimentales de extinción óptica con la teoría desarrollada en el Capítulo 2. Para el caso de NPs no esféricas se implementó el código de aproximación por dipolos discretos (DDA) para determinar la distribución espacial de campo en forma numérica. El mismo 
fue validado para el caso de NPs esféricas, comparándolo con los cálculos analíticos provenientes de teoría de Mie. Como aplicación, se muestra la determinación de la distribución de tamaño de NPs de sodio en una matriz de haluros alcalinos a través del ajuste del espectro de extinción.

El Capítulo 5 describe el refuerzo de campo de NPs esféricas con núcleo y doble cubierta, para lo cual se extendió la teoría de Mie al caso de NPs con 3 condiciones de contorno, determinando las expresiones de los coeficientes de los campos en cada una de las regiones. Los mayores refuerzos de campo obtenidos en los dieléctricos que conforman la NP compuesta, se explican a partir de la combinación de las resonancias de estructuras más simples, en base al modelo de hibridación. Finalmente utilizando la aproximación de dipolos discretos antes mencionada, conjuntamente con la teoría de Mie, se determina la sección eficaz de extinción de arreglos 1D de NPs de magnetita con una capa de dióxido de silicio, para la caracterización de cristales fotónicos sintonizables por campo magnético. Para esto se desarrolló un método novedoso que permite determinar la polarizabilidad equivalente y reducir drásticamente el tiempo de cálculo para sistemas complejos.

Adicionalmente a los 5 capítulos antes descritos, esta tesis consta de 4 apéndices, que contienen los detalles de cálculo de los aportes realizados. Estos apéndices tienen la finalidad de complementar el desarrollo de los 5 capítulos principales sin perder continuidad en la lectura de la idea central de los mismos. En el Apéndice A se deduce la expresión para la contribución de los electrones ligados a la función dieléctrica y se muestran gráficos correspondientes a la descripción de la función dieléctrica. En el Apéndice B se describe la teoría de Mie para NPs de núcleo y dos cubiertas, se describe la extinción de NPs con características magnéticas y se extiende a NPs de núcleo y dos cubiertas, la polarizabilidad debida a las corrientes de Eddy determinada por Landau. En el Apéndice C] se muestran los detalles del método de aproximación de dipolos discretos y por ultimo en el Apéndice D, se explican detalles de la teoría de hibridación aplicada a los plasmones en NPs esféricas. 


\section{Capítulo 2}

\section{Extinción, Scattering y absorción}

Intenta no volverte

un hombre de éxito, sino volverte

un hombre de valor.

Albert Einstein.

Cuando una onda electromagnética (OEM) interactúa con material particulado, una parte de aquella es dispersada hacia el medio en todas las direcciones (proceso conocido por su palabra en inglés "scattering") y otra parte es absorbida por el material. La suma de estos dos procesos produce una disminución de la intensidad original del haz en la dirección de incidencia, llamada "extinción". Esta interacción depende de parámetros asociados tanto al material particulado como a la OEM. En todos estos casos juega un rol especial el índice de refracción de los distintos medios involucrados. Éste depende de la permitividad dieléctrica y de la permeabilidad magnética del medio. Muchas veces, debido a que la permitividad depende de la frecuencia en un amplio rango que se extiende desde el UV hasta el lejano IR (FIR, por sus siglas en inglés), aquélla recibe el nombre de "función dieléctrica". La cantidad de energía que se absorbe y dispersa ("scatterea") depende, en términos generales, de la forma de las partículas dispersoras, del material o los materiales que la conforman, del medio en el cual se encuentran y de la frecuencia de la OEM incidente. Cuando las partículas son metálicas y de tamaño nanométrico, la función dieléctrica posee importantes variaciones con la longitud de onda y con el tamaño, por lo que es necesario tener un conocimiento preciso del comportamiento de aquélla para predecir las propiedades ópticas de coloides de NPs. 


\subsection{Modelado de la función dieléctrica}

Como es sabido, las propiedades de los materiales en escala nanométrica son diferentes de la que presentan en la escala macroscópica, hecho que los transforman en objeto de estudio para nuevas y desafiantes aplicaciones. En el caso particular de la respuesta óptica de NPs metálicas, es muy importante analizar el comportamiento de la función dieléctrica, que, además de presentar la conocida dependencia con la frecuencia, también resulta función del tamaño de la NP. Es necesario entonces establecer un modelo que describa adecuadamente estas dependencias. La estrategia desarrollada en esta tesis consiste en utilizar, como punto de partida, la función dieléctrica macroscópica compleja del metal (bulk) para luego incluir la dependencia con el tamaño. En términos generales, la función dieléctrica de un metal puede ser considerada como la suma de dos contribuciones: un término complejo proveniente de los electrones libres (transiciones intrabanda) y otro proveniente de los electrones ligados (transiciones interbanda) $21-23$ :

$$
\varepsilon(\omega)=\varepsilon_{\text {libres }}(\omega)+\varepsilon_{\text {lig }}(\omega)=\varepsilon^{\prime}(\omega)+i \varepsilon^{\prime \prime}(\omega)
$$

Estas contribuciones deben modificarse por tamaño para considerar, por un lado, la reducción del camino medio de los electrones libres por colisiones con la frontera de la partícula, y por el otro, la modificación de la distribución de niveles de energía a medida que el tamaño disminuye para el caso de los electrones ligados.

La contribución de los electrones libres en un metal se describe muy satisfactoriamente por el modelo de Drude, que considera al metal como un mar de electrones que pueden colisionar entre ellos y con la red cristalina, generando de esta manera un factor de amortiguamiento cuando se mueven forzados por el campo eléctrico armónico de la OEM incidente. En este marco, la ecuación de movimiento de los electrones está dada por la expresión:

$$
m^{*} \frac{d \vec{v}(t)}{d t}+\frac{m^{*} \vec{v}(t)}{\tau}=e \vec{E}_{0} e^{-i \omega t}
$$


donde el $m^{*}$ es la masa efectiva de los electrones, $\vec{v}(t)$ es la velocidad de arrastre de los mismos, $\vec{E}_{0}$ es la amplitud del campo eléctrico, $\tau$ es el tiempo medio entre colisiones de los electrones y $\omega$ es la frecuencia de la OEM incidente. La solución de esta ecuación tiene la forma $\vec{v}(t)=\vec{v}_{0} e^{-i \omega t}$, donde $\vec{v}_{0}$ es la amplitud de la velocidad. Recordando la ley de Ohm $\vec{j}=n e \vec{v}_{0}=\sigma \vec{E}_{0}$, donde $n$ es la densidad de electrones libres y $e$ es la carga del electrón, es posible encontar la expresión de la conductividad en función de la frecuencia:

$$
\sigma(\omega)=\frac{n e^{2} \tau}{m^{*}(1-i \omega \tau)}
$$

A partir de esta expresión, se obtiene la contribución de los electrones libres a la función dieléctrica macroscópica en términos de la frecuencia [24-31]:

$$
\varepsilon_{\text {libres }}(\omega)=1+\frac{i}{\omega \varepsilon_{0}} \sigma(\omega)=1-\frac{\omega_{p}^{2}}{\omega(\omega+i \gamma)}
$$

donde $\omega_{p}^{2}=\frac{n e^{2}}{m^{*} \epsilon_{0}}$ es la frecuencia de plasma y $\gamma=1 / \tau$ es la constante de amortiguamiento de los electrones libres debido a todos los procesos de colisión electrónelectrón, electrón-ion y electrón-fonón $1 / \tau=1 / \tau_{e-e}+1 / \tau_{e-i}+1 / \tau_{e-f}$.

Para evaluar la contribución de los electrones ligados a la función dieléctrica, algunos autores como Pinchuk [32] y Maier [33] consideran una constante real $\left(\chi_{\infty}\right)$, de modo que la función dieléctrica total quedaría de la forma:

$$
\varepsilon(\omega)=1-\frac{\omega_{p}^{2}}{\omega(\omega+i \gamma)}+\chi_{\infty}=\varepsilon_{\infty}-\frac{\omega_{p}^{2}}{\omega(\omega+i \gamma)} .
$$

Aunque esta expresión es muy utilizada en la literatura [4], no permite un ajuste completo de la función dieléctrica macroscópica experimental en un rango extendido de longitudes de onda, hecho que es imprescindible para explicar correctamente el comportamiento de los llamados plasmones, como se mostrará más adelante en este 
capítulo y en el 5 .

En esta tesis se avanzó en la descripción de la contribución de los electrones ligados a la función dieléctrica macroscópica compleja de metales nobles como por ejemplo Au [34] y $\mathrm{Cu}$ [35], tomando en cuenta la totalidad de las posibles transiciones interbanda entre la de valencia y la de conducción. Estas dependerán de la densidad de estados en la banda de conducción, de la distribución de estados no ocupados del estado superior y del perfil Lorentziano de las diferentes posibles transiciones interbanda.

Para este fin utilizamos dos aproximaciones: en primer lugar se consideró que las bandas de energía superiores son parabólicas y en segundo lugar se utilizó la aproximación de fase aleatoria (Random Phase Aproximation, RPA). Esta última se basa en la combinación de la suposición de planitud en la banda de valencia (aproximación de campo medio) y de la respuesta lineal del sistema. Así, la contribución de los electrones ligados a la función dieléctrica macroscópica, se describe como $34,36,37$ :

$$
\varepsilon_{l i g}(\omega)=K_{b} \int_{\omega_{g}}^{\infty} \frac{\sqrt{x-\omega_{g}}}{x}[1-F(x, T)] \frac{d x}{x^{2}-\omega^{2}+\gamma_{l i g}^{2}-2 i \omega \gamma_{l i g}}
$$

donde $E_{g}=\hbar \omega_{g}$ es la energía del gap, $F(x, T)$ es la función de distribución de Fermi (que depende de la frecuencia y de la energía de Fermi $E_{F}$ ), $K_{b}$ es una constante que depende del metal y $\gamma_{l i g}$ es la constante de amortiguamiento de los electrones ligados. En este caso la función dieléctrica macroscópica compleja total se puede expresar como:

$$
\varepsilon(\omega)=1-\frac{\omega_{p}^{2}}{\omega(\omega+i \gamma)}+K_{b} \int_{\omega_{g}}^{\infty} \frac{\sqrt{x-\omega_{g}}}{x}[1-F(x, T)] \frac{d x}{x^{2}-\omega^{2}+\gamma_{l i g}^{2}-2 i \omega \gamma_{l i g}}
$$

Cuando los valores de frecuencia superan escasamente la energía de transición interbanda (longitudes de onda largas), la energía de la OEM no alcanza a promover muchos electrones de la banda de valencia a la de conducción, haciendo que el tercer 
término de la ecuación 2.7, sea despreciable. En este caso, la función dieléctrica puede ser descripta sólo por los dos primeros términos que representan la contribución de los electrones libres (transiciones intrabanda del modelo de Drude).

$$
\varepsilon(\omega)=1-\frac{\omega_{p}^{2}}{\omega(\omega+i \gamma)}
$$

En la literatura encontramos medidas experimentales de la función dieléctrica correspondiente a diferentes metales como por ejemplo $\mathrm{Au}, \mathrm{Ag}, \mathrm{Cu}$ y $\mathrm{Al}$ realizadas por diferentes autores y en diferentes regiones del espectro electromagnético [21, 38-48]. De todas estas medidas, la más aceptada en la literatura corresponde a las realizadas por Johnson y Christy en 1972 [38]. Por otro lado, la más reciente corresponde a Babar 2015 [39], que extiende el rango de longitudes de onda al FIR. Ambas serán utilizadas en la próxima sección para determinar algunos parámetros de la función dieléctrica. Para describir la contribución de los electrones libres a la función dieléctrica se deben conocer los parámetros $\omega_{p}$ y $\gamma$ del modelo de Drude, tema que será abordado en la siguiente sección.

\subsubsection{Determinación de $\omega_{p} \mathbf{y} \gamma$}

Varios autores utilizan la aproximación $\omega \gg \gamma$ en el modelo de Drude [38, 49-51]. Esta condición permite obtener una relación líneal entre la parte real de $\varepsilon$ y $\lambda^{2}$ para determinar $\omega_{p}$, y entre la parte imaginaria de $\varepsilon$ y $\lambda^{3}$ para determinar $\gamma$. Recordando que $\omega=\frac{2 \pi c}{\lambda}$, las relaciones anteriores quedan de la forma:

$$
\epsilon^{\prime}(\lambda)=1-\frac{\omega_{p}^{2}}{4 \pi^{2} c^{2}} \lambda^{2} \quad \text { y } \quad \epsilon^{\prime \prime}(\lambda)=\frac{\omega_{p}^{2} \gamma}{8 \pi^{3} c^{3}} \lambda^{3},
$$

Sin embargo, los autores mencionados utilizaron la restricción $\omega \gg \gamma$ en el rango del espectro UV-IR cercano para el cual la función dieléctrica no se puede considerar sólo como la contribución de electrones libres (transiciones intrabanda), ya 
que existen contribuciones de electrones ligados (transiciones interbanda) que no pueden ser ignoradas.

Para solucionar esta contradicción en el cálculo de $\omega_{p}$ y $\gamma$, hemos desarrollado en este trabajo de tesis un método que, levantando la restricción anterior, permite deducir una relación lineal entre combinaciones de las partes real e imaginaria de la función dieléctrica de Drude, para valores experimentales de la misma en la región del espectro donde la contribución de los electrones ligados presenta poca o ninguna influencia. Dicha región corresponde a longitudes de onda largas, en la zona del IR mediano hasta el FIR. Así, trabajando algebraicamente la partes real e imaginaria de la función dieléctrica 2.8 , se pueden obtener las dos ecuaciones siguientes:

$$
\begin{gathered}
\omega \varepsilon^{\prime \prime}(\omega)=\gamma\left[1-\varepsilon^{\prime}(\omega)\right] \\
\omega^{2}\left[\left(\varepsilon^{\prime \prime}(\omega)\right)^{2}+\left(1-\varepsilon^{\prime}(\omega)\right)^{2}\right]=\omega_{p}^{2}\left[1-\varepsilon^{\prime}(\omega)\right],
\end{gathered}
$$

que, graficadas en función de $\left[1-\varepsilon^{\prime}(\omega)\right]$, representan dos rectas cuyas pendientes son $\gamma$ y $\omega_{p}^{2}$ respectivamente. La determinación de estos dos parámetros permite describir la función dieléctrica en este rango de longitudes de onda.

Si aplicamos el procedimiento antes descripto, utilizando los valores de las funciones dieléctricas experimentales dadas por Johnson y Christy [38] y Babar [39] para Au en los rangos de 188-1937 $\mathrm{nm}$ y 0.2066-12.40 $\mu \mathrm{m}$ respectivamente, se pueden graficar las ecuaciones 2.10 y 2.11 en función de $\left[1-\varepsilon^{\prime}(\omega)\right]$, obteniendo los datos representados por cuadrados azules y círculos rojos mostrados en las Figuras 2.1 (a) y (b).Se puede observar que, para cada uno de los gráficos, existe un conjunto de datos que pueden ajustarse con rectas cuyas pendientes permiten hallar $\omega_{p}^{2}$ y $\gamma$. Estas rectas ajustan los gráficos en el rango de longitudes de onda donde la influencia de los electrones ligados es despreciable. Las gráficas para determinar estos valores en los casos de Ag, Cu y Al, se muestran en el Apéndice $\mathbf{A}$. 

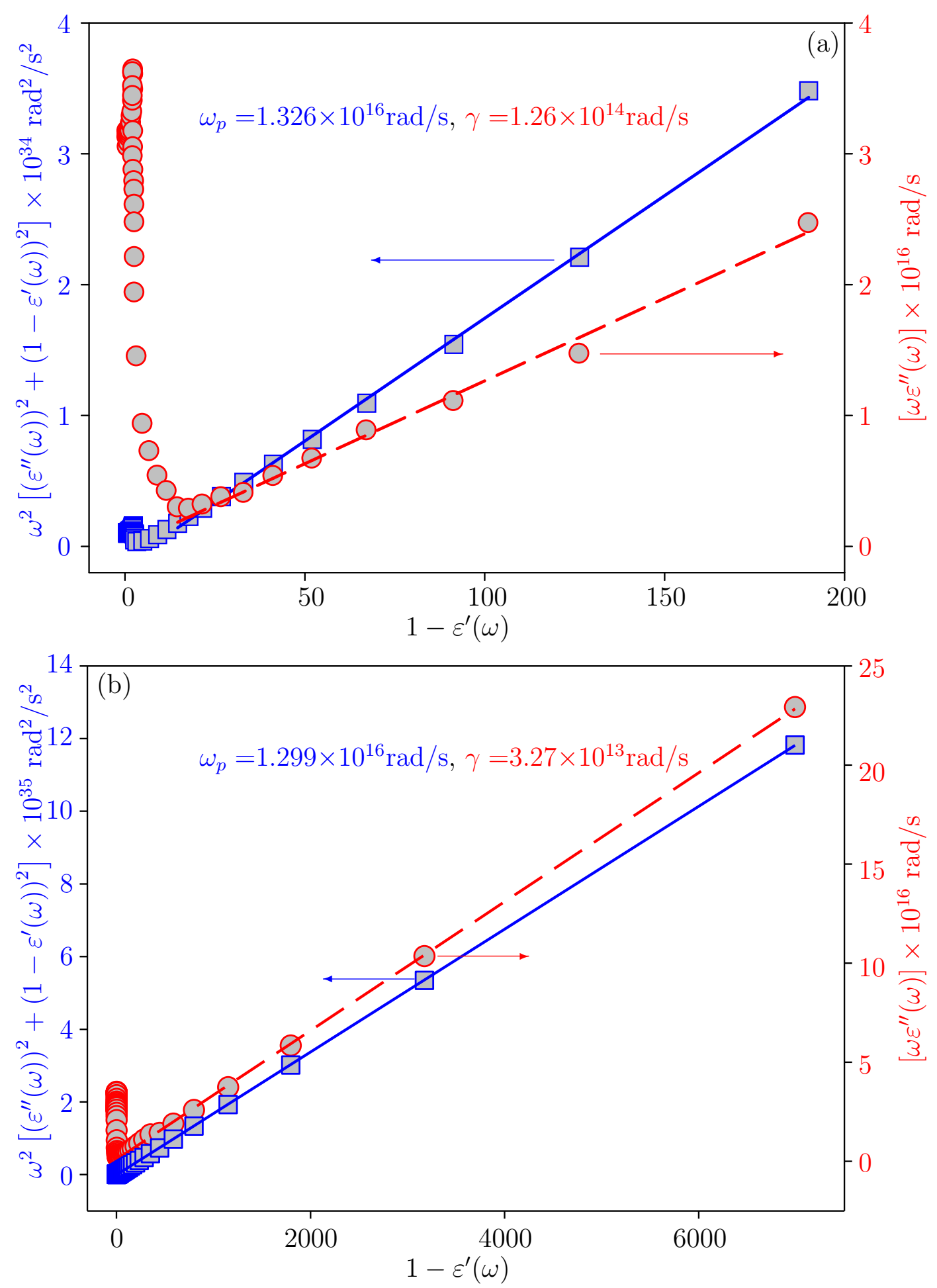

Figura 2.1: Gráficas de las ecuaciones 2.10 y 2.11 para Au (a) Johnson y Christy-1972 y (b) S.Babar-2015. Ajuste de los datos por regresión lineal para el rango donde la función dieléctrica es descrita por el modelo de Drude. Las pendientes obtenidas por la regresión lineal representan $\omega_{p}^{2}$ (línea llena) y $\gamma$ (línea segmentada). 
Los resultados obtenidos en esta tesis para cada uno de los metales estudiados, se resumen junto con los obtenidos por Rakic [21], Johnson y Christy [38] y Ordal [52] en la Tabla 2.1. La primera columna muestra las referencias de donde provienen los valores experimentales de la función dieléctrica bulk que fueron utilizados para calcular $\omega_{p}$ y $\gamma$. La segunda y tercera columnas muestran los resultados obtenidos para estos parámetros tanto por nosotros como por otros autores, y en la cuarta columna las referencias donde fueron publicados.

\begin{tabular}{|c|c|c|c|}
\hline Metal (Referencia) & $\omega_{p} \times 10^{15} \mathbf{r a d} / \mathrm{s}$ & $\gamma \times 10^{13} \mathbf{r a d} / \mathrm{s}$ & Referencia \\
\hline בAu (Johnson \& Christy, [38]) & $13.623 \pm 0.021$ & $\overline{12.234 \pm 0.040}$ & Mendoza et al. 53 \\
\hline $\mathrm{Au}$ (Babar, [39]) & 13.26 & 12.6 & Mendoza et al. $\overline{54}$ \\
\hline $\mathrm{Au}$ (Johnson \& Christy, [38]) & 13.70 & 10.70 & Johnson \& Christy 38$]$ \\
\hline Ag (Johnson \& Christy, 38 ) & $13.884 \pm 0.007$ & $3.197 \pm 0.025$ & Mendoza et al. $5 \overline{3}$ \\
\hline Ag (Babar, [39]) & 13.74 & 2.501 & Mendoza et al. \\
\hline Ag (Johnson \& Christy, 38]) & 13.93 & 3.220 & Johnson \&Christy 38 \\
\hline $\mathrm{Cu}$ (Johnson \& Christy, [38]) & $13.939 \pm 0.014$ & $14.06 \pm 0.014$ & Mendoza et al. \\
\hline $\mathrm{Cu}($ Babar, $[39])$ & 13.389 & 14.04 & Mendoza et al. $\overline{54}$ \\
\hline $\mathrm{Cu}$ (Johnson \& Christy, 38,) & 13.44 & 14.50 & Johnson-Christy 38 \\
\hline $\mathrm{Al}$ (McPeack, 40]) & $19.656 \pm 0.059$ & $17.073 \pm 0.690$ & Mendoza et al. 53 \\
\hline $\mathrm{Al}$ (Rakic, 21 ) & 17.437 & 23.846 & Mendoza et al. $\overline{\overline{54}}$. \\
\hline $\mathrm{Al}$ (Ordal, 52 ) & 17.46 & 7.687 & Ordal 52 \\
\hline $\mathrm{Al}$ (Rakic, 21.) & 16.46 & 7.141 & Rakic 21 \\
\hline
\end{tabular}

Tabla 2.1: Valores $\omega_{p}$ y $\gamma$ determinados en este trabajo para $\mathrm{Au}, \mathrm{Ag}, \mathrm{Cu}$ y $\mathrm{Al}$. 1ra columna: Metal (Función dieléctrica macroscópica experimental)

Se observa que los valores de $\omega_{p}$ determinados en esta tesis para los 4 metales estudiados son muy cercanos a los obtenidos por los autores antes mencionados, mientras que los valores de $\gamma$ presentan importantes discrepancias. Una forma de analizar la bondad de los valores de $\omega_{p}$ y $\gamma$ determinados en esta tesis, consiste en analizar su capacidad de ajuste a la función dieléctrica experimental, teniendo en cuenta que sólo se considera en esta etapa la contribución de los electrones libres. Este ajuste se muestra en línea continua en la Figura 2.2 para el Au, observándose que la función dieléctrica obtenida con los parámetros determinados en este trabajo ajusta mucho mejor los valores experimentales que cuando se utilizan los valores de $\omega_{p}$ y $\gamma$ determinados por Rakic y Johnson y Christy [21, 38]. 

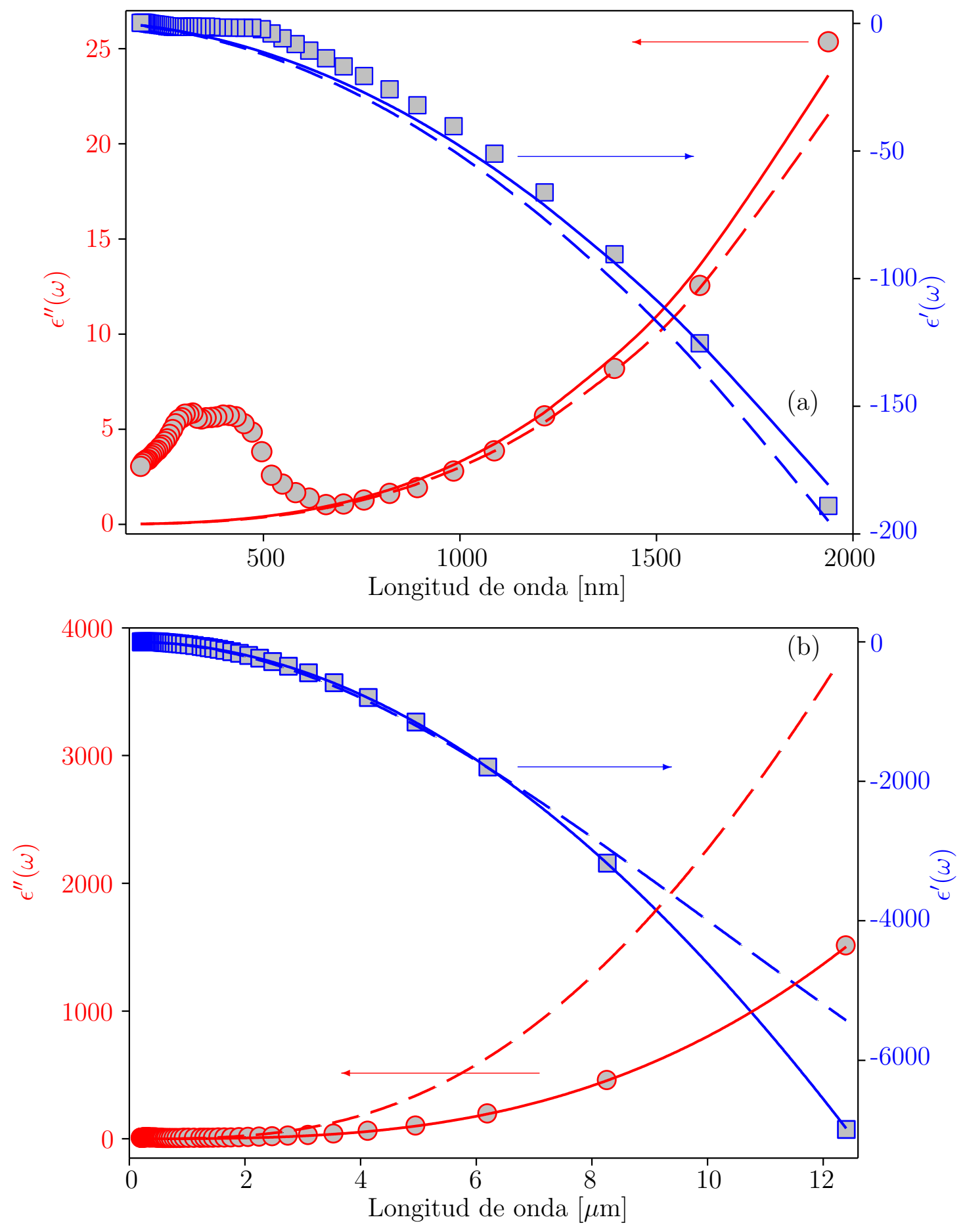

Figura 2.2: Partes real e imaginaria de la contribución de los electrones libres a la función dieléctrica experimental del Au (a) Johnson y Christy y (b) Babar. En líneas continuas se muestran los ajustes realizados utilizando los valores de $\omega_{p}$ y $\gamma$ obtenidos de 2.10 y 2.11, mientras que en líneas segmentadas se muestran los ajustes utilizando los valores de $\omega_{p}$ y $\gamma$ dados por Johnson y Christy. Los cuadrados y círculos grises representan los valores de las partes real e imaginaria de la función dieléctrica experimental. 
Una causa del mejor ajuste obtenido con nuestro método puede hallarse en el hecho de que el rango de medida de la función dieléctrica utilizado por Babar es mucho más extendido hacia el FIR que el utilizado por Johnson y Christy. En esa zona de longitudes de onda largas, los electrones ligados presentan menor influencia que en la zona utilizada por Johnson y Christy y por tanto la descripción dada por el modelo de Drude es más apropiada y muy cercana a los valores experimentales. Para longitudes de ondas más cortas (entre $200 \mathrm{~nm}$ y $700 \mathrm{~nm}$ aproximadamente) la descripción del modelo de Drude es inadecuada, como se observa en la Figura 2.2. a. Esto se debe a que falta considerar la contribución de los electrones ligados a la función dieléctrica, tema que será abordado más adelante en este mismo capítulo.

Una situación similar puede observarse cuando se analiza el caso del Al. En la Figura 2.3 puede verse que el modelo de Drude ajusta muy bien los valores experimentales determinados por Ordal [52] para longitudes de onda mayores a 1400 nm aproximadamente, mientras que existe una fuerte discrepancia en la región cercana a los $800 \mathrm{~nm}$ donde los electrones ligados presentan una gran influencia causada por la presencia de bandas paralelas en la estructura de bandas de este metal [55, 56].

De las comparaciones anteriores, se puede concluir que las constantes $\omega_{p}$ y $\gamma$ determinadas por el procedimiento implementado en esta tesis, sintetizado en las ecuaciones 2.10 y 2.11, permiten calcular una función dieléctrica que reproduce correctamente la obtenida en forma experimental para longitudes de onda largas, donde existe poca influencia de la contribución de los electrones ligados. Esto sugiere que el novedoso procedimiento desarrollado posee la capacidad de determinar las constantes $\omega_{p}$ y $\gamma$ con muy buena precisión sin necesidad de utilizar la aproximación $\omega \gg \gamma$.

Como puede verse de las figuras anteriores, queda claro que para reproducir completamente la función dieléctrica, se debe determinar e incluir apropiadamente la contribución de los electrones ligados. Un método directo para esta determinación consiste en restar a la función dieléctrica experimental de volumen, la contribución de los electrones libres (representada por el modelo de Drude) determinada con los valores de $\omega_{p}$ y $\gamma$ obtenidos anteriormente y mostrados en la Tabla 2.1. 

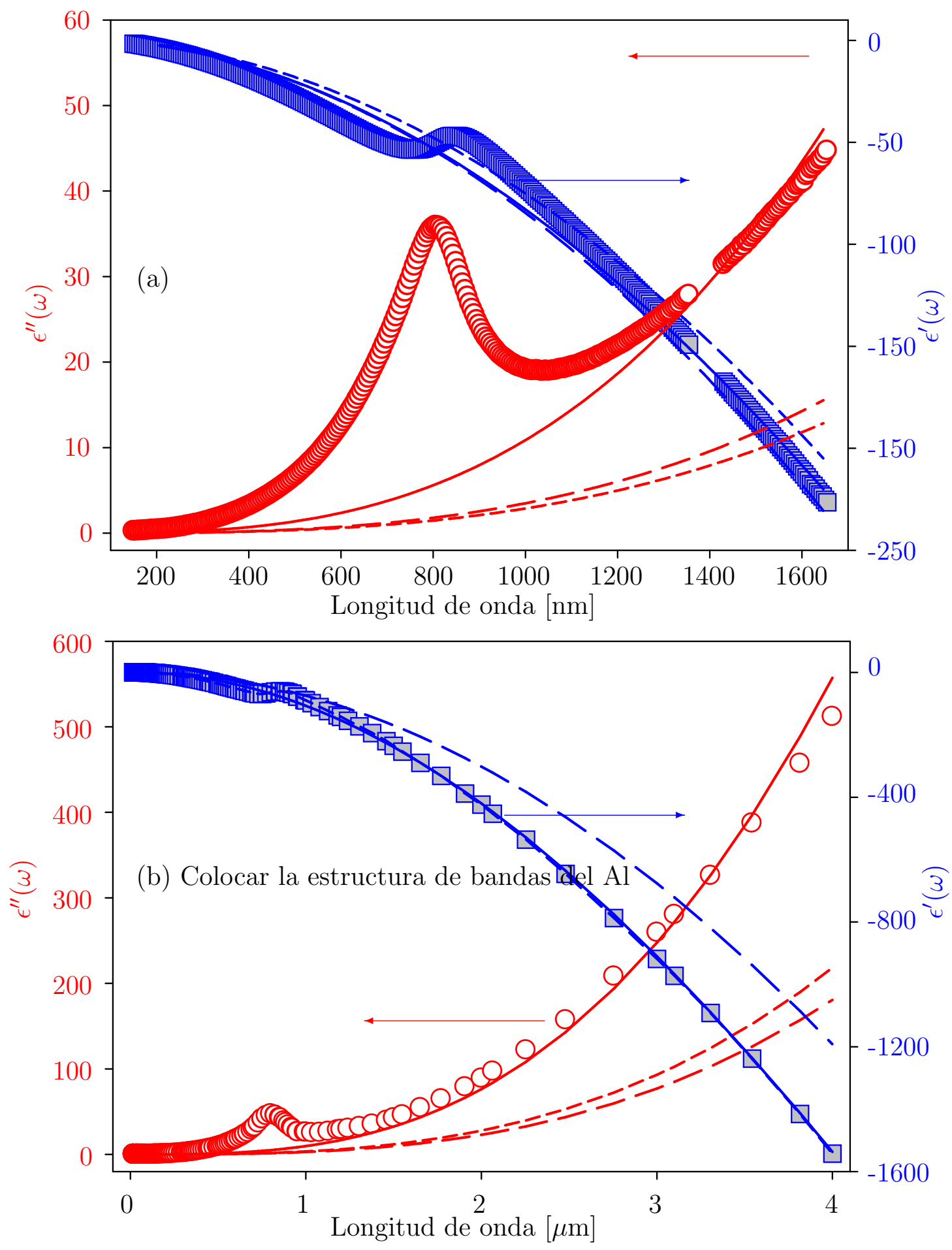

Figura 2.3: Partes real e imaginaria de la contribución de los electrones libres a la función dieléctrica (a) Al K. M. McPeak-2015 y (b) Al A. D. Rakic-1998, utilizando los valores de $\omega_{p}$ y $\gamma_{\text {libres }}$ obtenidos de las ecuaciones $(\mathbf{2 . 1 0})$ y $(\mathbf{2 . 1 1})$ en línea continua, utilizando los valores dados en P. B. Johnson-1972 para el $\mathrm{Al}$ en segmentos largos y los valores dados en A. D. Rakic-1998 y M.A. Ordal-1988 en segmentos largos y cortos respectivamente para el Al. Los cuadrados azules y círculos rojos representan las partes real e imaginaria de la función dieléctrica experimental. 
Si el metal cumple satisfactoriamente con la aproximación RPA (como el Au y el $\mathrm{Cu}$, por ejemplo), es posible determinar los parámetros que describen la contribución de los electrones ligados a la función dieléctrica a partir de la ecuación 2.6, como fue realizado por Inouye et al. [34], Santillán et al. [35], Scaffardi et al. [57]y Santillán et al. [58, 59] y que se resumen en la Tabla 2.2.

\begin{tabular}{ccccc}
\hline \hline Metal & $E_{g}[\mathbf{e V}]$ & $E_{F}[\mathbf{e V}]$ & $\gamma_{\text {lig }} \times 10^{14}[\mathbf{r a d} / \mathbf{s}]$ & $K_{b} \times 10^{24}\left[\mathbf{r a d}^{1,5} / \mathbf{s}^{1,5}\right]$ \\
\hline \hline $\mathrm{Au}[$ referen $]$ & $2.1[$ referen $]$ & $2.5[$ referen $]$ & 2.4 & 2.3 \\
$\mathrm{Cu}$ & 1.95 & 2.15 & 1.15 & 2.0 \\
\hline \hline
\end{tabular}

Tabla 2.2: Parámetros que describen la contribución de los electrones ligados a la función dieléctrica del $\mathrm{Au}$ y del $\mathrm{Cu}$, utilizando la aproximación RPA.

En el caso del Al, que presenta una estructura de bandas que no permite aplicar la aproximación RPA, la contribución de los electrones ligados puede ser representada por la sumatoria descripta por Rakic [21]:

$$
\varepsilon_{l i g}(\omega)=\sum_{j=1}^{k} f_{j}\left[\frac{\omega_{p}^{2}}{\omega_{j}^{2}-\omega^{2}+\gamma_{j}^{2}-2 i \omega \gamma_{j}}\right]
$$

donde $f_{j}, \omega_{j}$ y $\gamma_{j}$ representan la fuerza de oscilador, la frecuencia de resonancia y la constante de amortiguamiento de la j-ésima transición considerada, respectivamente. Esta expresión surge de un modelo semicuántico para interpretar el movimiento de los electrones en metales, cuyo resultado se asemeja al modelo clásico de Lorentz para materiales dieléctricos.

Para el caso del Al es suficiente considerar hasta el tercer término de la ecuación 2.12 para encontrar una buena coincidencia entre los valores experimentales y la correspondiente descripción teórica de la función dieléctrica en la region de intéres $(0.2 \mu \mathrm{m}-4 \mu \mathrm{m})$. Los valores de los parámetros correspondientes a los tres primeros términos de la sumatoria que ajustan los datos experimentales de la función dieléctrica se resumen en la Tabla 2.3. En la misma se ha realizado la sustitución $\omega_{p j}^{2}=f_{j} \omega_{p}^{2}$. 


\begin{tabular}{cccc}
\hline \hline Orden & $\omega_{p j} \times 10^{15} \mathrm{rad} / \mathrm{s}$ & $\omega_{j} \times 10^{15} \mathrm{rad} / \mathrm{s}$ & $\gamma_{j} \times 10^{15} \mathrm{rad} / \mathrm{s}$ \\
\hline \hline 1 & 4.13 & 0.608 & 0.72 \\
2 & 7.05 & 2.356 & 0.68 \\
3 & 7.95 & 2.965 & 2.10 \\
\hline \hline
\end{tabular}

Tabla 2.3: Parámetros que describen la contribución de los electrones ligados a la función dieléctrica del Al.

En la Figura 2.4 se muestra en línea continua la parte real y en segmentos largos la parte imaginaria de la función dieléctrica del $\mathrm{Al}$ considerada como la suma de las contribuciones de los electrones libres y ligados. En cuadrados y círculos se representan los valores experimentales de las partes real e imaginaria, respectivamente.

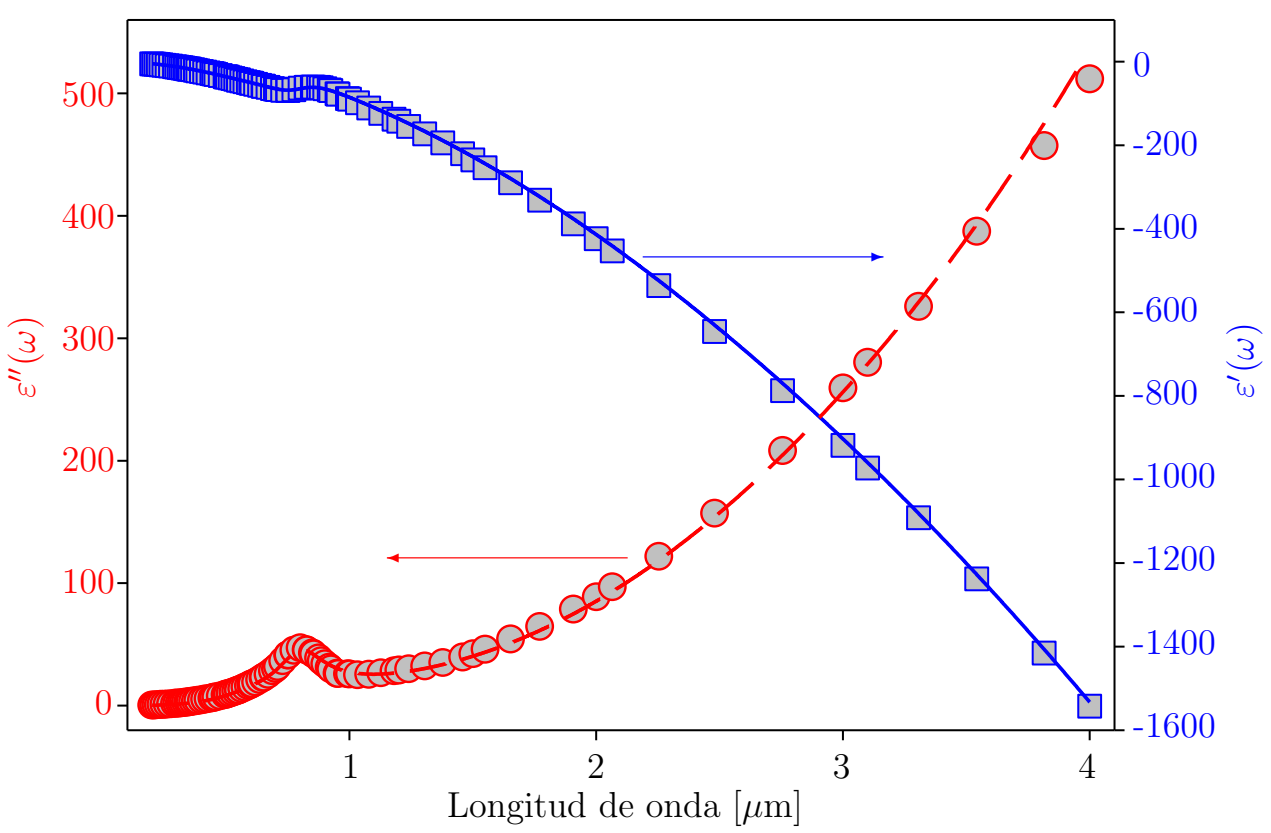

Figura 2.4: Partes real (línea continua) e imaginaria (línea segmentada) de las contribuciones de los electrones libres y ligados a la función dieléctrica del $\mathrm{Al}$, utilizando los valores de $\omega_{p} \mathrm{y} \gamma$ obtenidos en esta tesis y los parámetros dados en la Tabla 2.3 . Los cuadrados y círculos representan las partes real e imaginaria de la función dieléctrica experimental, respectivamente.

El conocimiento de la contribución de los electrones ligados a la función dieléctrica de volumen (como se verá en la siguiente sección) toma preponderancia cuando se requiere corregir la función dieléctrica por tamaño para NPs de radio inferior a $2 \mathrm{~nm}$ aproximadamente dependiendo del metal. 


\subsubsection{Corrección de la función dieléctrica por tamaño}

Como hemos mencionado en la sección anterior, la función dieléctrica de los metales depende de la frecuencia y está conformada por dos contribuciones: la de los electrones libres y la de los electrones ligados. Para introducir el efecto del tamaño desde el modelo de Drude, se deben considerar las colisiones de los electrones libres con la superficie del metal, además de las colisiones de los electrones con la red y entre sí mismos. Esto se traduce en una disminución del camino libre medio de los electrones o, lo que es lo mismo, una disminución del tiempo medio entre eventos de colisión. Desde el punto de vista del modelo de Drude, esto se traduce en un mayor amortiguamiento en el movimiento de los electrones. Por esta razón, se suele hacer una corrección a la constante de amortiguamiento $\gamma$ para tener en cuenta las colisiones de los electrones con la frontera del metal. Si consideramos que la NP metálica es esférica de radio $R$, se suele sumar a la constante $\gamma$ un término que depende inversamente del radio, dado por la expresión 60-65]:

$$
\gamma_{c}(R)=\gamma+C \frac{v_{F}}{R}
$$

donde $v_{F}$ es la velocidad de Fermi, $R$ es el radio de la NP esférica y $C$ es una constante cuyo valor típico es del orden de 3/4. Esta corrección suele ser suficiente para modificar la función dieléctrica de NPs hasta tamaños del orden de $10 \mathrm{~nm}$.

En cuanto a la contribución de electrones ligados, la corrección por tamaño debe tomar en cuenta la separación de niveles de energía producida por la disminución del tamaño de la NP. Para ello se debe modificar el factor $K_{b}$ de la contribución de electrones ligados por una expresión dependiente del radio $K_{b}(R)$ 35, 57- 59 :

$$
K_{b}(R)=K_{b}\left(1-e^{-R / R_{0}}\right)
$$

donde $R_{0}$, es una constante que depende del metal y en el caso de los metales estudiados su valor es cercano a 0,35 nm. Con estas modificaciones, la función dieléctrica dependiente de la frecuencia y del tamaño de una NP esférica, se puede representar por: 


$$
\begin{aligned}
\varepsilon(\omega, R)= & 1-\frac{\omega_{p}^{2}}{\omega\left(\omega+i \gamma_{c}(R)\right)} \\
& +\left(1-e^{-R / R_{0}}\right) K_{b} \int_{\omega_{g}}^{\infty} \frac{\sqrt{x-\omega_{g}}}{x}[1-F(x, T)] \frac{d x}{x^{2}-\omega^{2}+\gamma_{l i g}^{2}-2 i \omega \gamma_{l i g}},
\end{aligned}
$$

Esta ecuación puede ser reescrita en la forma:

$$
\begin{aligned}
\varepsilon(\omega, R)= & 1-\frac{\omega_{p}^{2}}{\omega\left(\omega+i \gamma_{c}(R)\right)}-\frac{\omega_{p}^{2}}{\omega(\omega+i \gamma)}+\frac{\omega_{p}^{2}}{\omega(\omega+i \gamma)} \\
& +K_{b} \int_{\omega_{g}}^{\infty} \frac{\sqrt{x-\omega_{g}}}{x}[1-F(x, T)] \frac{d x}{x^{2}-\omega^{2}+\gamma_{l i g}^{2}-2 i \omega \gamma_{l i g}} \\
& -e^{-R / R_{0}} K_{b} \int_{\omega_{g}}^{\infty} \frac{\sqrt{x-\omega_{g}}}{x}[1-F(x, T)] \frac{d x}{x^{2}-\omega^{2}+\gamma_{l i g}^{2}-2 i \omega \gamma_{l i g}}
\end{aligned}
$$

Esta expresión general de la función dieléctrica dependiente del tamaño puede ser escrita como la función dieléctrica experimental macroscópica $\varepsilon_{v}(\omega)$, definida por las ecuaciones 2.7 y 2.12 (suma del primero, tercero y quinto término de 2.16), que es independiente del tamaño de la NP, más dos correcciones por tamaño, una para la contribución de los electrones libres $\Delta \varepsilon_{l i b}(\omega, R)$ y otra para la contribución de los electrones ligados $\Delta \varepsilon_{\text {lig }}(\omega, R)$.

$$
\begin{aligned}
\varepsilon(\omega, R)= & \varepsilon_{v}(\omega)+\frac{\omega_{p}^{2}}{\omega(\omega+i \gamma)}-\frac{\omega_{p}^{2}}{\omega\left(\omega+i \gamma_{c}(R)\right)} \\
& -e^{-R / R_{0}} K_{b} \int_{\omega_{g}}^{\infty} \frac{\sqrt{x-\omega_{g}}}{x}[1-F(x, T)] \frac{d x}{x^{2}-\omega^{2}+\gamma_{l i g}^{2}-2 i \omega \gamma_{l i g}}= \\
= & \varepsilon_{v}(\omega)+\Delta \varepsilon_{l i b}(\omega, R)+\Delta \varepsilon_{l i g}(\omega, R)
\end{aligned}
$$

donde 


$$
\begin{aligned}
\Delta \varepsilon_{l i b}(\omega, R) & =\frac{\omega_{p}^{2}}{\omega(\omega+i \gamma)}-\frac{\omega_{p}^{2}}{\omega\left(\omega+i \gamma_{c}(R)\right)} \\
\Delta \varepsilon_{l i g}(\omega, R) & =-e^{-R / R_{0}} K_{b} \int_{\omega_{g}}^{\infty} \frac{\sqrt{x-\omega_{g}}}{x} \frac{[1-F(x, T)] d x}{x^{2}-\omega^{2}+\gamma_{l i g}^{2}-2 i \omega \gamma_{l i g}}
\end{aligned}
$$

Como se dijo anteriormente, la contribución de los electrones ligados a la función dieléctrica se puede representar como la diferencia entre la función dieléctrica de volumen y la contribución de los electrones libres. Con esta consideración la corrección por tamaño para la contribución de los electrones ligados $\Delta \varepsilon_{l i g}(\omega, R)$ se puede escribir como:

$$
\Delta \varepsilon_{l i g}(\omega, R)=-e^{-R / R_{0}}\left(\varepsilon_{v}(\omega)-1+\frac{\omega_{p}^{2}}{\omega(\omega+i \gamma)}\right)
$$

Utilizando los valores de $\omega_{p}$ y $\gamma$, reportados en la Tabla 2.1 más los valores de $v_{F}$, se puede calcular la función dieléctrica de los metales para diferentes frecuencias y tamaños de la NP considerada. Estos cálculos se muestran para el Au en la Figura 2.5 en el rango de longitudes de onda entre 200 y $2000 \mathrm{~nm}$ y para los radios de 2, 5, 10, 20 y $50 \mathrm{~nm}$. Para otros metales estudiados se muestran los resultados en el Apéndice A. Para todos ellos, los cambios en función del tamaño de la parte imaginaria de la función dieléctrica presentan mayor variación que para la parte real. Para todos los metales, cuando el radio de la NP se aproxima a $50 \mathrm{~nm}$, las partes real e imaginaria de la función dieléctrica se comportan como la función dieléctrica macroscópica.

En la Figura 2.6 se muestra una comparación entre la función dieléctrica de volumen del Au más la corrección de la contribución de los electrones libres (círculos, cuadrados y triángulos negros) y la misma función dieléctrica pero incluyendo la corrección de los electrones ligados en línea continua y (círculos, cuadrados y triángulos grises), para $0.7,1.0$ y $2.0 \mathrm{~nm}$ de radio respectivamente. 

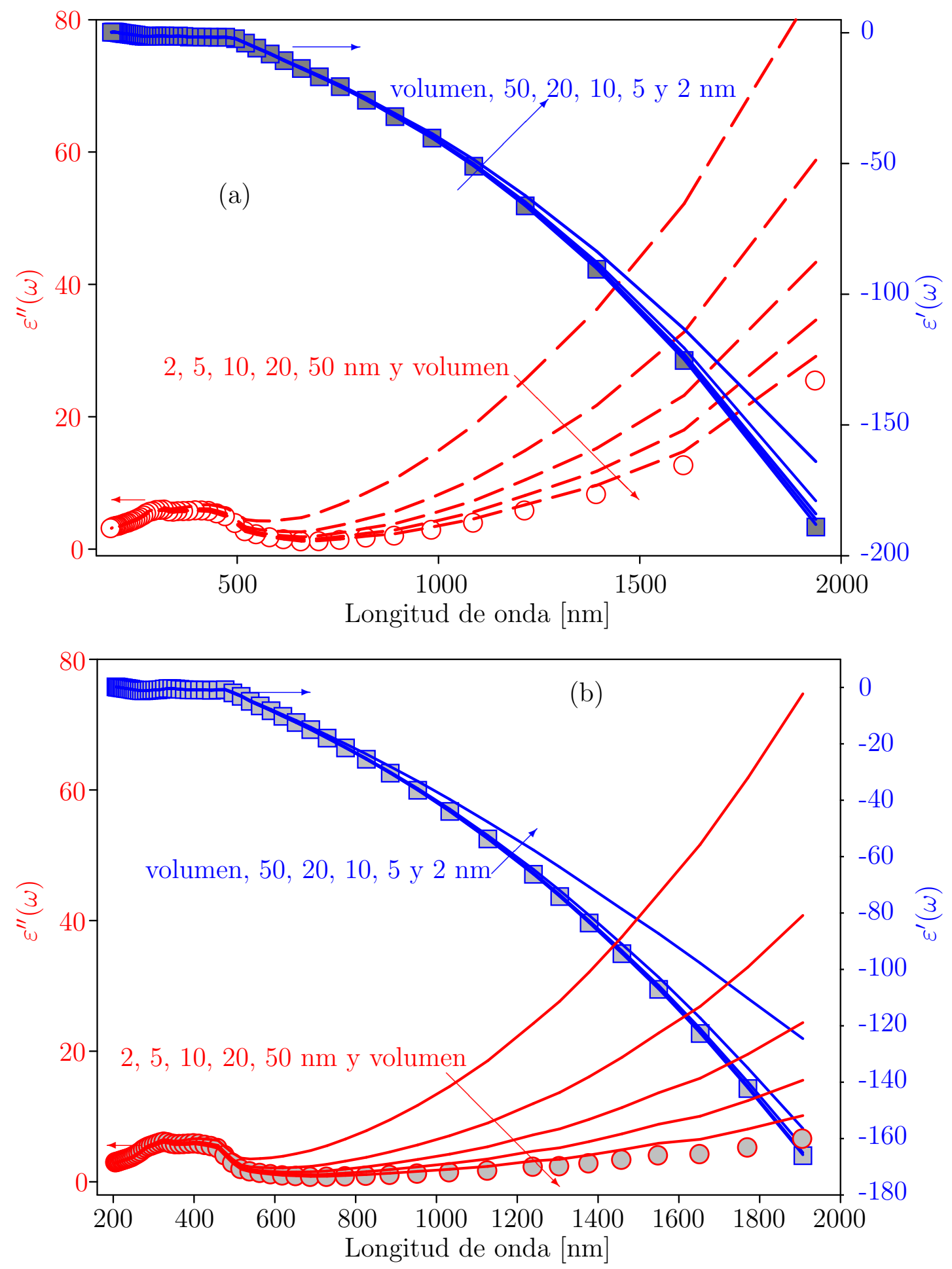

Figura 2.5: Partes real (línea continua) e imaginaria (línea segmentada) de la contribución de los electrones libres a la función dieléctrica de $\mathrm{Au}$ (a) Johnson \& Christy y (b) Babar, para diferentes radios de nanopartículas. Los cuadrados grises y círculos representan las partes real e imaginaria de la función dieléctrica experimental. 

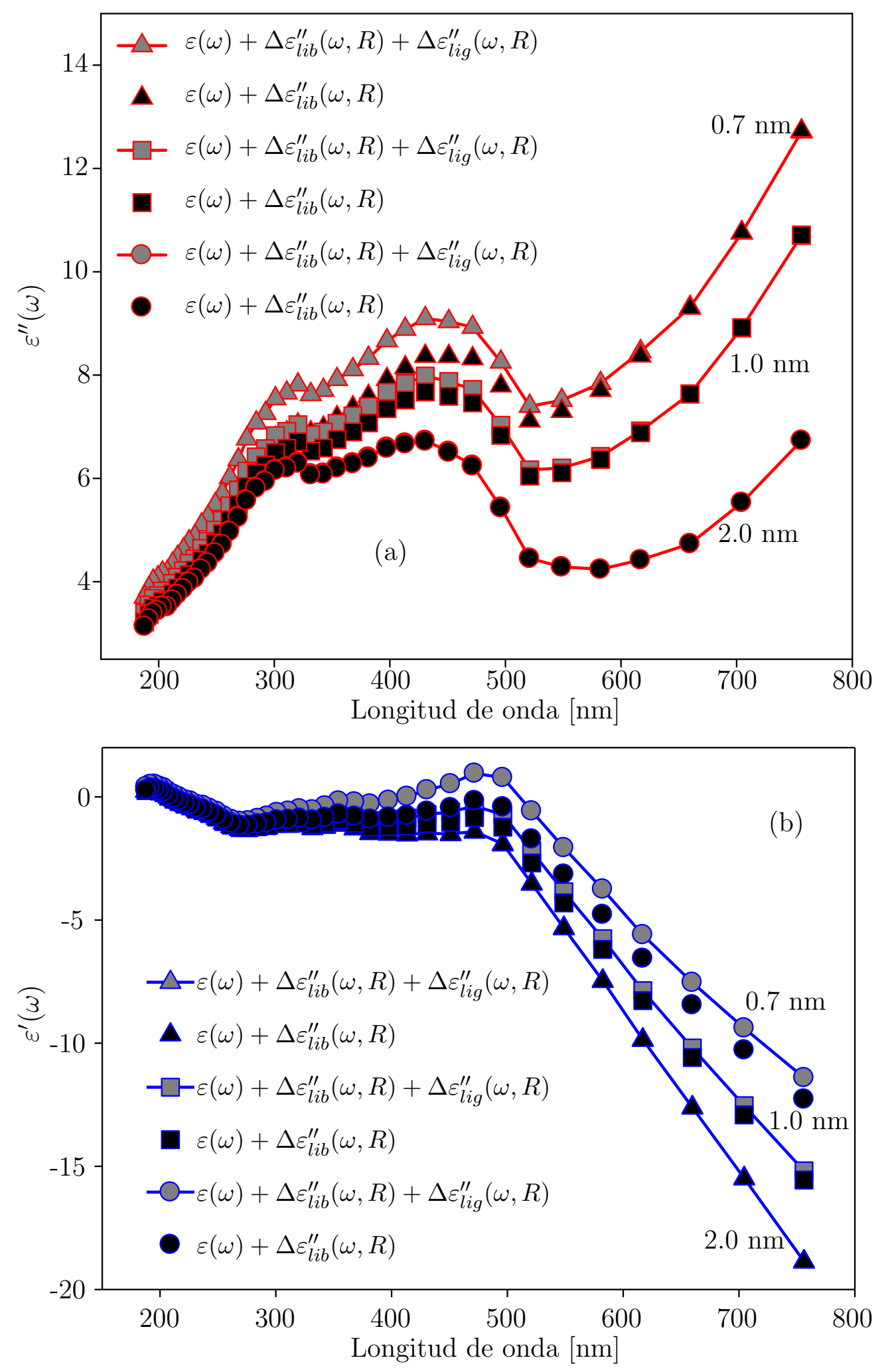

Figura 2.6: Partes imaginaria (a) y real (b). Suma de la función dieléctrica de volumen más la corrección de la contribución de los electrones libres (triángulos, cubos y círculos negros) y la suma de las correcciones de las contribuciones de los electrones libres y ligados (triángulos, cubos y círculos grises) para radios de 2,1 y $0.7 \mathrm{~nm}$ respectivamente. 
Puede verse que la corrección de los electrones ligados resulta relevante solo para radios de la NP inferiores a $2 \mathrm{~nm}$ aproximadamente mientras que la corrección de la contribución de los electrones libres es importante para radios de NP inferiores a 50 nm aproximadamente ver Figura 2.5. A su vez la importancia de la corrección de la contribución de los electrones ligados es más relevante a medida que la longitud de onda es mas pequeña.

Considerando que para NPs por encima de $2 \mathrm{~nm}$ la corrección de la contribución de los electrones ligados a la función dieléctrica es muy pequeña, se puede escribir la función dieléctrica para NPs de radios superiores a $2 \mathrm{~nm}$ como:

$$
\varepsilon(\omega, R)=\varepsilon_{v}(\omega)+\frac{i \omega \omega_{p}^{2}\left(\gamma_{c}(R)-\gamma\right)}{\left(\omega^{2}+i \omega \gamma\right)\left(\omega^{2}+i \omega \gamma_{c}(R)\right)}=\varepsilon_{v}(\omega)+\Delta \varepsilon_{l i b}(\omega, R)
$$

\subsection{Permeabilidad magnética}

Para completar la descripción del índice de refracción de NPs, se debe conocer, además de la función dieléctrica, la permeabilidad magnética de las mismas. Los materiales que presentan momentos magnéticos, poseen una importante dependencia de la permeabilidad magnética con la frecuencia. En un material magnético, la magnetización y el campo aplicado están relacionados por medio de la permeabilidad magnética. Para conocer esta última, se debe calcular la magnetización resolviendo la ecuación de Gilbert

$$
\frac{d \mathbf{M}}{d t}=\gamma \mathbf{M} \times \mathbf{H}_{T}+\alpha_{G} \frac{\mathbf{M}}{|\mathbf{M}|} \times \frac{d \mathbf{M}}{d t}
$$

donde $\mathbf{M}$ es la magnetización, $\gamma=-g e /\left(2 m_{e} c\right)$ es la relación entre el momento magnético y el momento angular, $g=2$ es el factor giromagnético, e y $m_{e}$ son la carga y masa del electrón, $c$ es la velocidad de la luz, $\alpha_{G}$ es el parámetro de Gilbert y $H_{T}$ es el campo total efectivo. 
Uno de los compuestos que mejor representa a este tipo de materiales es la magnetita $\left(\mathrm{Fe}_{3} \mathrm{O}_{4}\right)$. El interés en este tipo de óxidos ha crecido debido, entre otras propiedades, a su capacidad para formar NPs de tipo núcleo-cubierta. La magnetita tiene una temperatura de ordenamiento $T_{N}=860 \mathrm{~K}$ y una magnetización de saturación a temperatura ambiente $M_{s}=90 \mathrm{Am}^{2} / \mathrm{kg}[66,67]$. Este compuesto tiene una estructura de espín cúbica inversa donde dos tercios de los iones de $\mathrm{Fe}$ son $\mathrm{Fe}^{3+}$. La mitad de estos iones ocupan sitios A (sitios tetraédricos) y la otra mitad ocupan sitios B (sitios octaédricos). El tercio restante de iones de $\mathrm{Fe}_{\text {son }} \mathrm{Fe}^{2+}$ y ocupan sitios B. Dado que los momentos $\mathrm{Fe}_{A}^{3+}$ y $\mathrm{Fe}_{B}^{3+}$ se compensan, la magnetización de saturación, $m_{A}+m_{B}=4 \mu_{B}$ f.u. (formula unidad) es superior a la de los restantes momentos $\mathrm{Fe}_{B}^{2+}$.

Para un campo armónico de la forma $\mathbf{H}=H_{0} e^{i \omega t}$, donde $H_{0}$ es la amplitud del campo y considerando polarización circular, Draine \& Hensley [68] deducen una expresión para la permeabilidad de la forma:

$$
\mu(\omega)=1+\frac{12 \pi\left(\chi_{+}+\chi_{-}\right)}{9-4 \pi\left(\chi_{+}+\chi_{-}\right)}
$$

donde

$$
\chi_{ \pm}=\frac{2 N_{A B} \omega_{M A} \omega_{M B}-\omega_{M A} \omega_{0 B}^{\prime}-\omega_{M B} \omega_{0 A}^{\prime} \pm\left(\omega_{M A}+\omega_{M B}\right) \omega}{N_{A B}^{2} \omega_{M A} \omega_{M B}-\omega_{0 A}^{\prime} \omega_{0 B}^{\prime}-\omega^{2} \pm\left(\omega_{0 A}^{\prime}+\omega_{0 B}^{\prime}\right) \omega}
$$

$\operatorname{con} \omega_{0 A}^{\prime}=\omega_{0 A}+i \alpha_{G} \mathrm{y} \omega_{0 B}^{\prime}=\omega_{0 B}-i \alpha_{G}$. Para la magnetita, $\omega_{0 A}=-2,18 \times 10^{14}$ $\mathrm{rad} / \mathrm{s}, \omega_{0 B}=1,21 \times 10^{14} \mathrm{rad} / \mathrm{s}, \omega_{M A}=2,52 \times 10^{13} \mathrm{rad} / \mathrm{s}, \omega_{M B}=2,01 \times 10^{13} \mathrm{rad} / \mathrm{s} \mathrm{y}$ $N_{A B}=1,08 \times 10^{4}$.

El valor del parámetro $\alpha_{G}$ para la magnetita se puede obtener de los valores experimentales de la permeabilidad magnética. Kong et al [69] determinaron experimentalmente la permeabilidad magnética efectiva $\mu_{e}$ de compuestos de NPs de $\mathrm{Fe}_{3} \mathrm{O}_{4}$ en un gel termoplástico natural (NP/TPNR) para tres concentraciones (4 wt \%, 8 wt \% y 12 wt \%). Estos resultados se han utilizado en este trabajo de tesis para determinar el valor del parámetro $\alpha_{G}$.

Debido a que la permeabilidad medida por Kong et al [69] corresponde a un 
compósito de magnetita y no a un material puro, debemos utilizar la descripción de Bruggeman modificada por Rousselle et al. [70,71], para determinar la permeabilidad efectiva :

$$
p \frac{A \mu_{i}-\mu_{e}}{A \mu_{i}+2 \mu_{e}}=(p-1) \frac{\mu_{m}-\mu_{e}}{\mu_{m}+2 \mu_{e}}
$$

donde $p$ es la fracción volumen de las NPs magnéticas, $\mu_{i}$ es la permeabilidad de las NPs, $\mu_{m}$ es la permeabilidad del medio, $\mu_{e}$ es la permeabilidad efectiva y $A$ es un parámetro de la forma:

$$
A=2 \frac{k R \cos k R-\sin k R}{\sin k R-k R \cos k R-k^{2} R^{2} \sin k R},
$$

con el número de onda dado por

$$
k=[1+i]\left(\frac{\pi f \mu_{i}}{\rho \varepsilon_{0} c^{2}}\right)^{1 / 2},
$$

donde $f$ es la frecuencia, $\rho$ es la resistividad eléctrica y $R$ es el radio de la NP.

Utilizando las ecuaciones 2.23 2.27, se puede modelar la permeabilidad magnética efectiva de los compuestos en función de los parámetros $\alpha_{G} \mathrm{y}$ wt \%.

En la Figura 2.7 se muestran los resultados correspondientes a las partes real e imaginarias de la permeabilidad magnética efectiva de compuestos de NP/TPNR para dos concentraciones diferentes (4 wt \% y 8 wt \%) y diferentes valores de $\alpha_{G}(0.1,0.3$ y 2.0). Las NPs caracterizadas por Kong presentan radios en el rango entre $[20,30] \mathrm{nm}$. 

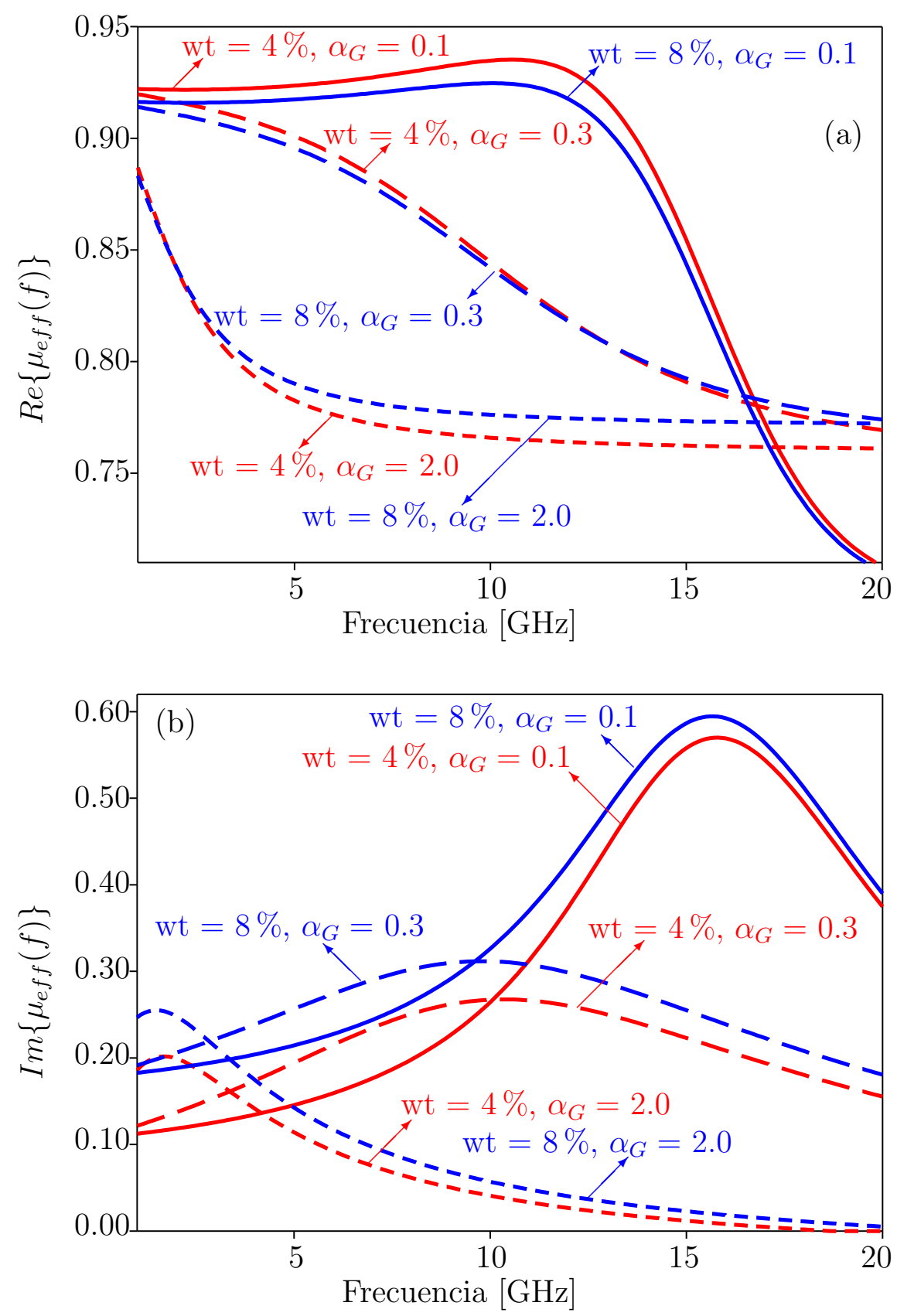

Figura 2.7: Partes real (a) e imaginarias (b) de la permeabilidad magnética efectiva del compuesto de magnetita en TPR para diferentes valores del parámetro de Gilbert $\alpha_{G}=\left[\begin{array}{lll}0.1 & 0.3 & \text { y } 2.0\end{array}\right]$ y diferentes valores de la relación peso-peso wt $\%$ de 4 y $8 \%$. 
El valor de $\alpha_{G}$ para cada una de las concentraciones se obtuvo en este trabajo de tesis por minimización del error cuadrático entre los cálculos teóricos y los puntos experimentales. El promedio de los resultados es 0.32 que corresponde al valor finalmente utilizado.

En la Figura 2.8 se muestra la comparación entre los valores experimentales y calculados para $8 \mathrm{wt} \%$. Se observa un muy buen acuerdo entre los valores experimentales y los calculados para las partes real e imaginaria de la permeabilidad magnética en el rango $[1,20] \mathrm{GHz}$.

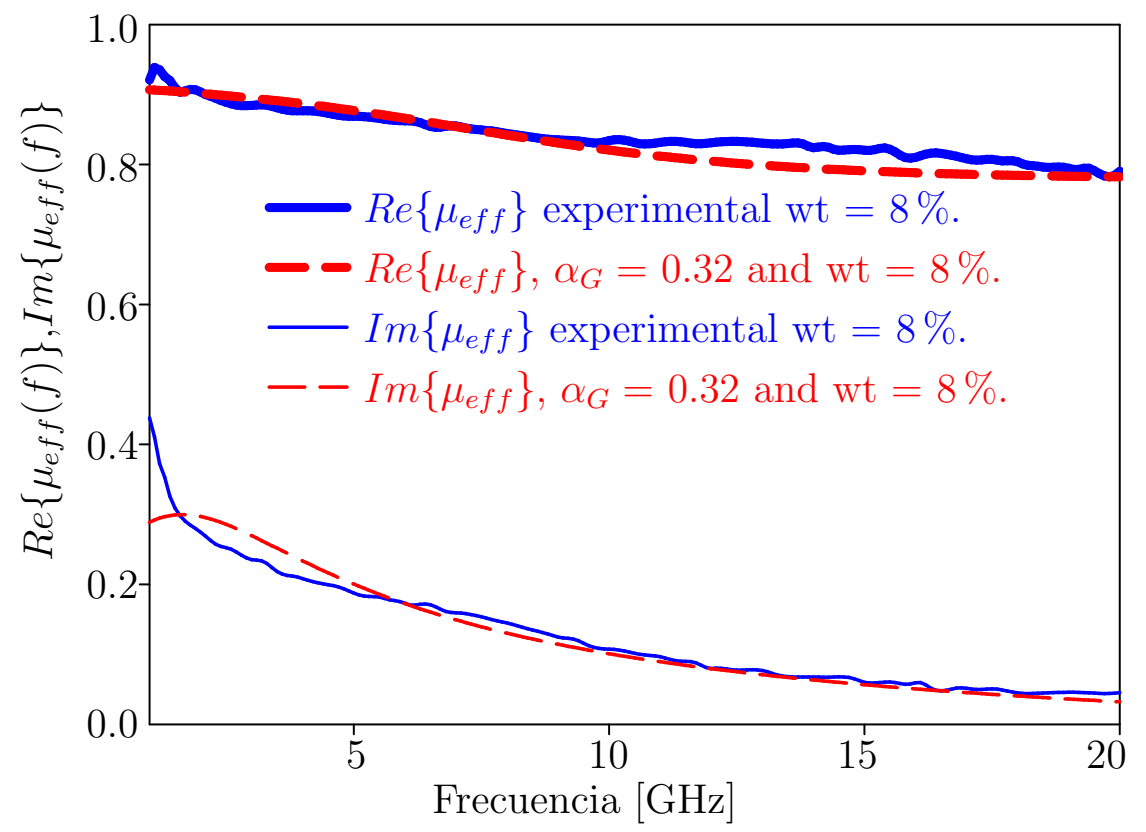

Figura 2.8: Comparación entre los valores experimentales para $\mathrm{wt}=8 \%$ [19] y valores teóricos con $\alpha_{G}=0.32$ de la permeabilidad magnética efectiva de magnetita.

\subsection{Extinción de nanopartículas sin cubierta}

La extinción para NPs esfericas depende del radio de la NP (en el caso de NPs sin cubierta del radio $a$ y en el caso de NPs núcleo-cubierta de los radios $a$ y $b$ ), del índice de refracción de cada uno de los medios que forma la NP (en el caso de NPs núcleo-cubierta de los índices de refracción del núcleo y la cubierta) y del medio circundante. 
En las secciones anteriores describimos adecuadamente el índice de refracción como una función de la frecuencia y del radio de la NP. Para estudiar los espectros experimentales de las NPs debemos conocer los efectos de cada uno de los parametros que influyen en la extinción.

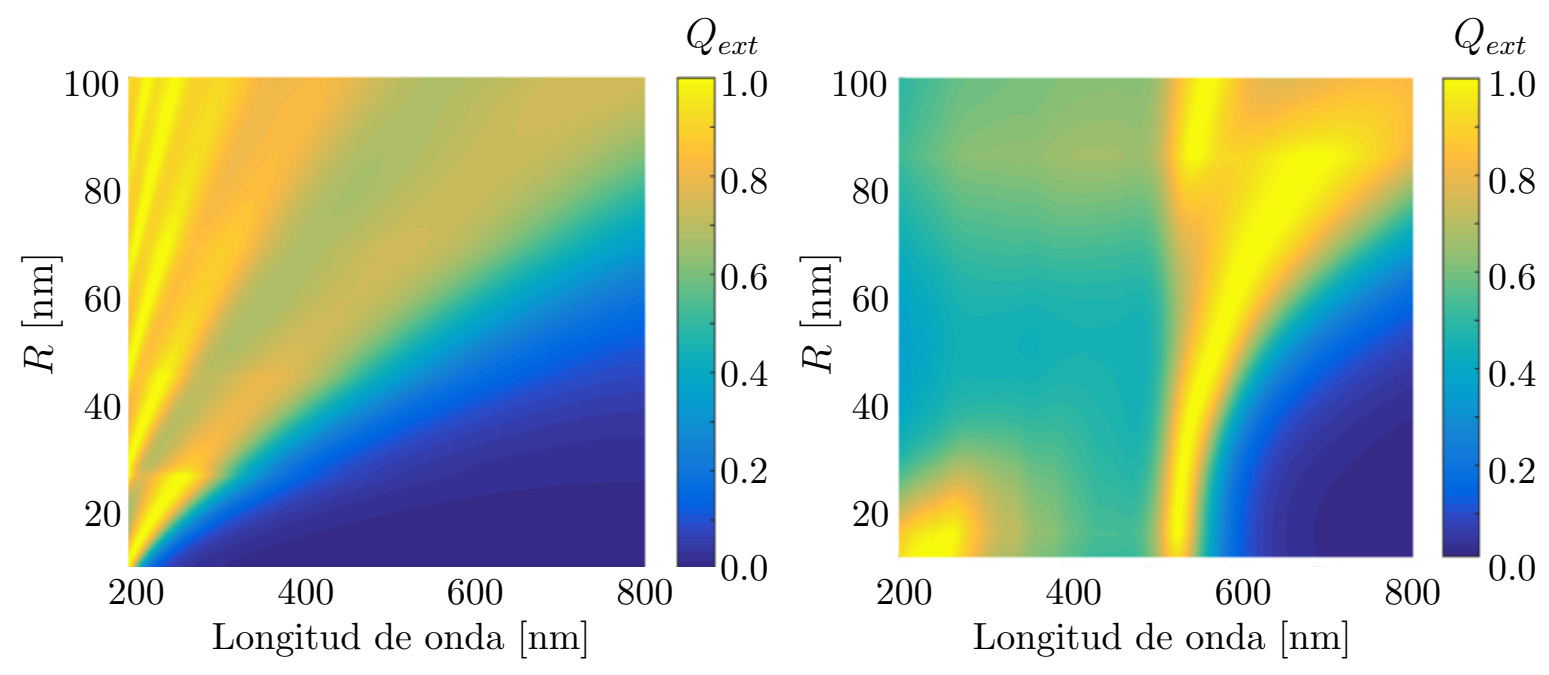

Figura 2.9: Extinción de NPs con núcleo de $\mathrm{Al}$ a la izquierda y Au a la derecha, para diferentes radios de las NPs

Uno de los factores más importantes en los espectros de extinción de NPs esféricas plasmónicas, corresponde al hecho de que cuando se incrementa el radio de la NP, el pico plasmónico se ensancha y desplaza hacia longitudes de onda más largas. Adicionalmente al incrementar el tamaño las contribuciones de orden superior (cuadrupolares, octopolares, etc.) adquieren una importancia relevante en la extinción de las NPs. Estas conclusiones se observan en la Figura 2.9 para el caso de Nps de Al y $\mathrm{Au}$. Es interesante notar que las contribuciones multipolares aparecen para tamaños menores en el caso del $\mathrm{Al}$ comparado con el $\mathrm{Au}$, lo que muestra que la aproximación dipolar se puede utilizar en un rango mas amplio en el caso del Au con respecto al Al.

\subsection{Extinción de nanopartículas con una cubierta}

Cuando las NPs son metálicas, existe la posibilidad de que el medio en el cual se encuentran por ejemplo el agua promueva la oxidación. En caso de considerar como 
metal el Al una de las posibles especies corresponde a NPs de Al con una cubierta de óxido de $\mathrm{Al}$ (donde el más usual es el $\mathrm{Al}_{2} \mathrm{O}_{3}$ ).

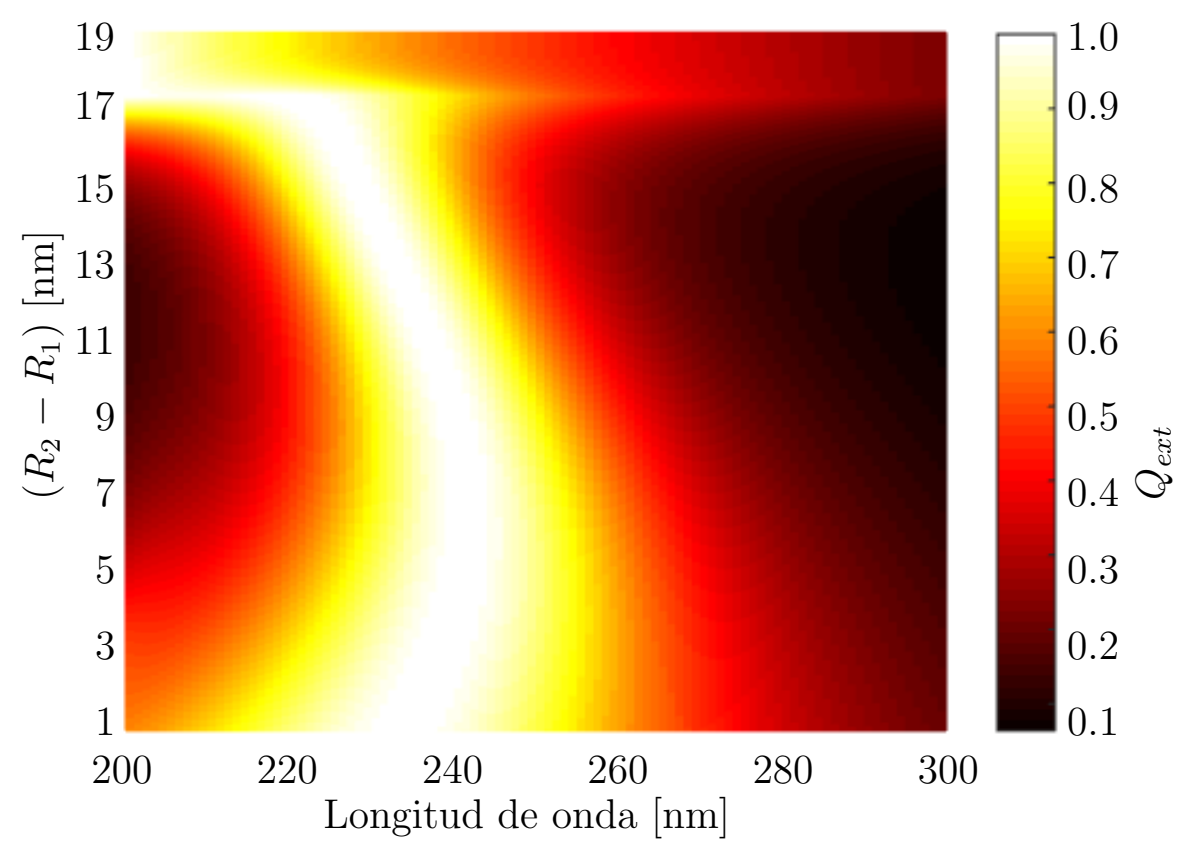

Figura 2.10: Extinción de NPs con núcleo de $\mathrm{Al}$ y cubierta de $\mathrm{Al}_{2} \mathrm{O}_{3}$ para diferentes espesores de la cubierta, el radio total de la NP es $R_{2}=20 \mathrm{~nm}$

Para estas NPs del tipo núcleo-cubierta se pueden calcular espectros de extinción, obteniendo la Figura 2.10, donde se observa que a medida que la cubierta del óxido formada es mas gruesa, el pico del plasmón se desplaza hacia longitudes de onda más largas. Esto ocurre hasta valores del espesor cercanos $7 \mathrm{~nm}$, después de lo cual el pico plasmónico se desplaza nuevamente hasta valores menores de longitud de onda, llegando al hecho de que cuando la NP se encuentra totalmente oxidada no existe pico plasmónico.

Las NPs de núcleo de aire o de $\mathrm{Al}_{2} \mathrm{O}_{3}$, con una cubierta de $\mathrm{Al}$ al igual que las NPs de $\mathrm{Al}$ con una cubierta de $\mathrm{Al}_{2} \mathrm{O}_{3}$, cambian la ubicación del pico plasmónico conforme cambia el espesor de la cubierta.

En la Figura 2.11, se muestra la variación del espectro de extinción de NPs con núcleo de $\mathrm{Al}_{2} \mathrm{O}_{3}$ cubierta por $\mathrm{Al}$, cuyo radio total es de $20 \mathrm{~nm}$. En este caso se varía el espesor de la cubierta de $\mathrm{Al}$ y a medida que el espesor de la cubierta disminuye, el pico del plasmónico se desplaza a longitudes de onda más largas, con una sensibilidad muy 


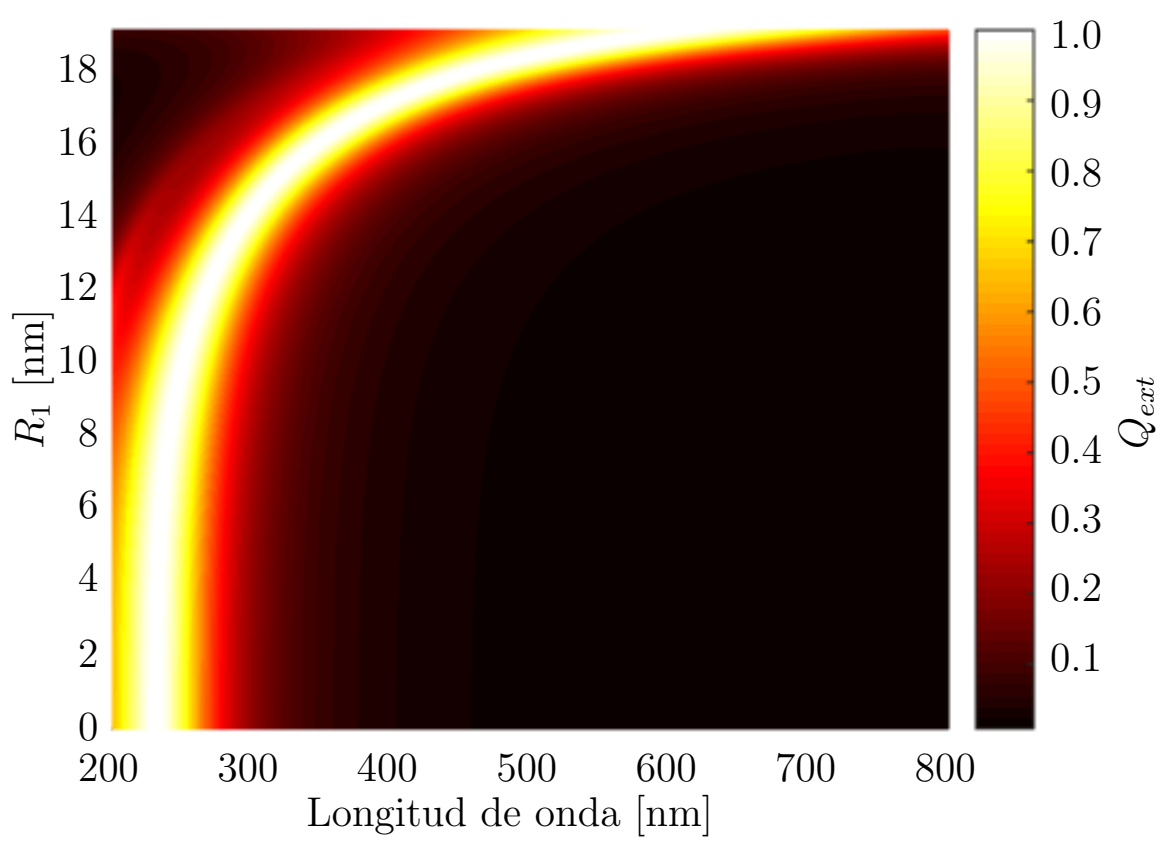

Figura 2.11: Espectros de Extinción de NPs con núcleo de $\mathrm{Al}_{2} \mathrm{O}_{3}$ y cubierta de $\mathrm{Al}$ para diferentes espesores de la cubierta; el radio total de las NPs es de $20 \mathrm{~nm}$

alta a la variación del espesor de la cubierta.

Uno de los modelos mas utilizados para describir la oxidación de metales es el propuesto por Mott-Cabrera [72]. Una mejora de este modelo, para su aplicación en NPs es propuesta por Litrico et. al. [73] donde se describe la evolución de la capa de óxido por medio de un sistema de ecuaciones diferenciales:

$$
\begin{array}{r}
\frac{d a}{d t}=\Omega n \nu \exp \left\{-\frac{U}{k T_{p}}+\frac{q \delta \Phi_{m}}{k T_{p}(b-a)}\right\} \\
m_{p} c_{p} \frac{d T_{p}}{d t}=\dot{Q}_{q u i}-\dot{Q}_{r a d}-\dot{Q}_{c o n v}
\end{array}
$$

donde $a$ es el espesor de la capa de óxido, $\Omega$ es el volumen vacante por ion desplazado, $n$ es el número de iones por unidad de área en la posición de salto por encima de la barrera de energía $U, \nu$ es la frecuencia de intento de saltos iónicos, $k$ es la constante de Boltzmann, $T_{p}$ es la temperatura de la NP que se asume uniforme en toda la NP dado que sus dimensiones son pequeñas, $\Phi_{m}$ es el potencial de Mott en la capa de óxido, $\delta$ es la distancia entre un máximo y un mínimo adyacentes de 
la barrera de energía, $m_{p}$ es la masa de la NP, $c_{p}$ es el calor especifico de la NP. Como la NP esta formada por metal y óxido del metal, como por ejemplo $\mathrm{Al}$ y $\mathrm{Al}_{2} \mathrm{O}_{3}$ $m_{p} c_{p}=m_{A l} c_{A l}+m_{\mathrm{Al}_{2} \mathrm{O}_{3}} c_{\mathrm{Al}_{2} \mathrm{O}_{3}}$. $\dot{Q}_{q u i}$ es el calor químico producido por la reacción Al-O, que es proporcional a la tasa de crecimiento del espesor del oxido multiplicado por la entalpía de la oxidación $\dot{Q}_{q u i}=4 \pi a^{2} \Delta h_{o x} \frac{d a}{d t}$. $\dot{Q}_{\text {rad }} \mathrm{y} \dot{Q}_{c o n v}$ son las perdidas por radiación y convección respectivamente. Descritos por la ley de Stefan-Boltzmann $\epsilon \sigma A_{p}\left(T_{p}^{4}-T_{m}^{4}\right)$ y la ecuación propuesta por Fuchs $74 \dot{Q}_{c o n v}=4 \alpha \pi b^{2} P_{02} \sqrt{\frac{k T_{f}}{8 \pi m_{02}}} \frac{\gamma+1}{\gamma-1}\left(\frac{T_{p}}{T_{f}}-1\right)$, donde $\epsilon$ es la emisividad $\sigma$ es la constante de Stefan-Boltzmann, $T_{m}$ es la temperatura del medio circundante, $T_{f}$ es la temperatura de la frontera considerada como el promedio entre la temperatura del medio circundante y la temperatura de la NP, $\gamma$ es la razón de calor especifico, $m_{02}$ es la masa de la molécula de gas , $P_{02}$ es la presión parcial del gas y $\alpha$ es el coeficiente de acomodación.

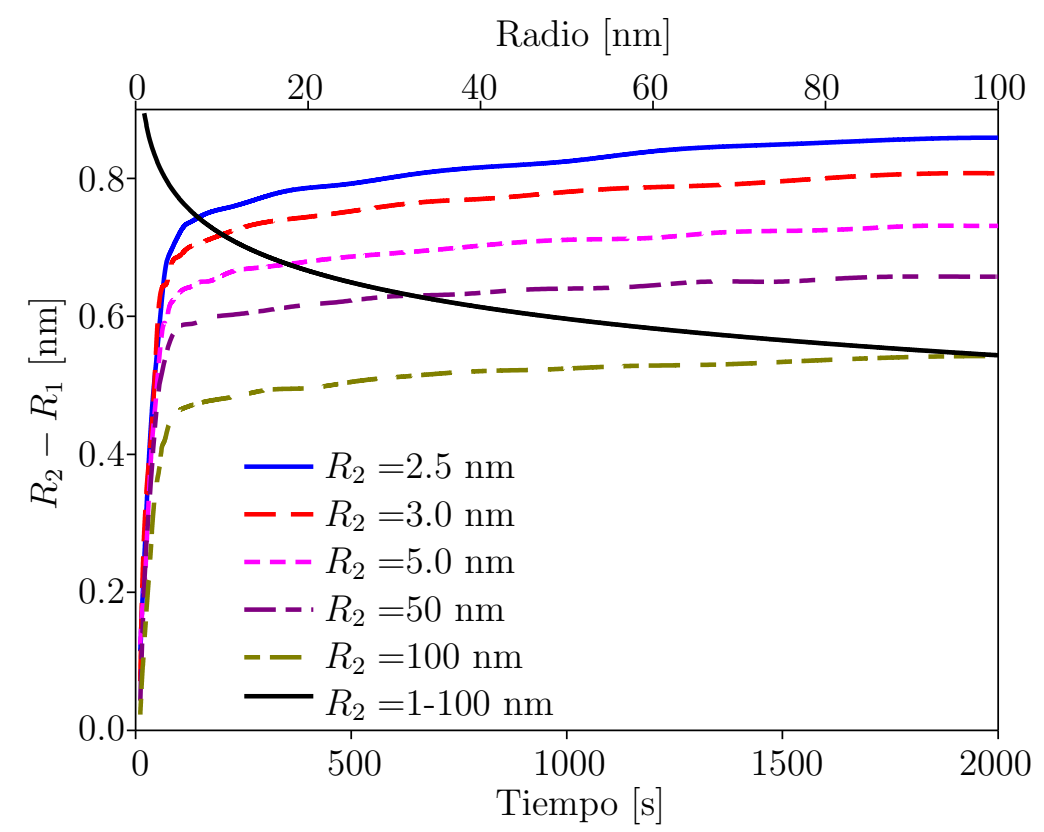

Figura 2.12: Evolución de la oxidación de NPs de Al para diferentes radios de la NP.

La solución de este sistema por el método de Runge Kutta de cuarto orden nos da la evolución del espesor de la cubierta de óxido $\left(R_{2}-R_{1}\right)$ en función del tiempo para diferentes radios de la NP Figura 2.12, donde en línea negra se muestra la variación de la capa final de óxido en función del radio de la NP. Es importante notar que cuanto 
menor es el radio de la NP mayor es la capa de óxido que se forma.

Otro tipo de NPs que fue estudiada en esta tesis son las que presentan propiedades ópticas y magnéticas por tener una configuración núcleo-cubierta del tipo $\mathrm{Fe}_{3} \mathrm{O}_{4}-\mathrm{Au}$. En éstas juegan un papel importante el radio interior, el espesor de la cubierta y la distribución de tamaños de las NPs.

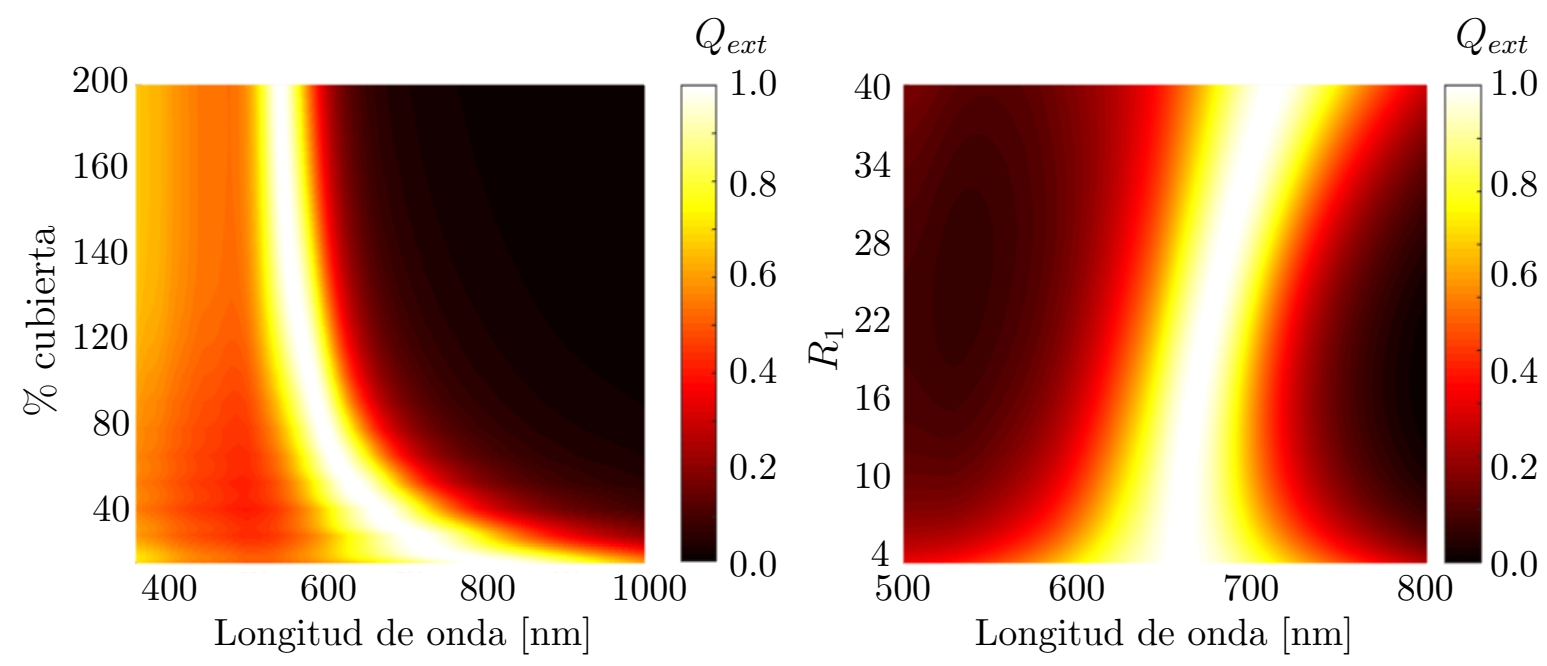

Figura 2.13: Gráfico de espectros de extinción para NPs esféricas de magnetita de: (a) $4 \mathrm{~nm}$ de radio y con una cubierta de Au para diferentes porcentajes de la cubierta. (b) diferentes radios y con una cubierta de Au del $40 \%$ del radio.

En la parte izquierda de la Figura 2.13, se muestran espectros de NPs de magnetita de $4 \mathrm{~nm}$ de radio con diferentes espesores de la nanocubierta de $\mathrm{Au}$, en este caso el valor de $\sigma$ utilizado es 0.001 (NPs monodispersas). Al incrementar el valor del espesor de la nanocubierta de Au el pico plasmónico se desplaza hacia longitudes de onda más cortas y el mismo se hace más angosto.

Otro parámetro importante en estas NPs es el incremento del tamaño de la NP. En la parte derecha de la Figura 2.13 se muestra el espectro de NPs de magnetita con una cubierta de Au correspondiente al $40 \%$ del radio del núcleo. Al incrementar el radio del núcleo y por ende el espesor de la nanocubierta, el pico plasmónico se desplaza hacia longitudes de onda largas y el contraste (relación máximo- mínimo del pico plasmónico) del mismo incrementa. 
Resumiendo, en este Capítulo 2 se ha desarrollado la formulación teórica necesaria para interpretar y modelar los resultados experimentales de espectros de extinción óptica de NPs esféricas generadas tanto por medios físicos como químicos. Este capítulo incluye el estudio de la dependencia del indice de refracción con la frecuencia y el radio de las NPs, para lo cual es necesario conocer los parámetros de Drude (frecuencia de plasma), (constante de amortiguamiento) y el coeficiente de Gilbert. Los parámetros de Drude para metales nobles y de transición fueron determinados con un novedoso método, que permite obtener su dependencia con la longitud de onda en rangos extendidos que cubren desde el UV hasta el NIR o FIR, para algunos metales. 
Capítulo 3

\section{Fabricación de nanopartículas}

\section{por ablación láser}

La constancia

es la virtud por la cual

todas las otras virtudes

dan fruto.

Arturo Graf.

Una de las áreas más estudiadas en nanotecnología es la producción de NPs de tamaños típicamente inferiores a $100 \mathrm{~nm}$. Las mismas pueden ser dieléctricas o metálicas y los métodos de síntesis son principalmente de tipo químico (pirólisis, micela inversa, sol-gel, etc.), deposición en fase gaseosa o mecánicos (molienda). Las técnicas químicas y las basadas en crecimiento epitaxial en fase gaseosa son los más tradicionales, aunque sufren el inconveniente de generar compuestos no deseados las primeras y necesitar de instrumental sofisticado la segunda. La técnica de molienda posee el inconveniente de generar contaminación en el estado final de la muestra. Para el caso de Nps metálicas se han explorado en los últimos años distintos métodos de fabricación con el objeto de aumentar la pureza y la variedad de las mismas.

La técnica de ablación láser pulsada surge como una alternativa interesante ya que puede ser aplicada a una variedad casi ilimitada de materiales y solventes, ofreciendo varias ventajas comparativas, como la de no necesitar precursores químicos (y por lo tanto obtener soluciones coloidales de alta pureza) y la de generar NPs esféricas de tamaño relativamente pequeño, entre otras. 
Se entiende por ablación láser a la eyección de cantidades microscópicas de material de la superficie de un sólido, inducida por interacción con láseres pulsados intensos (aprox. $10^{6}$ a $10^{14} \mathrm{~W} / \mathrm{cm}^{2}$ ) y de corta duración (aprox. $10^{-13}$ a $10^{-8} \mathrm{~s}$ ). Esto puede ocurrir tanto en vacío, como en atmósfera gaseosa a diferentes presiones o en líquidos. Cuando la duración de los pulsos láser es mucho menor que la escala de tiempo de transferencia de energía de los electrones libres a la red cristalina del blanco sólido (del orden de los unos pocos picosegundos), la temperatura de ésta no alcanza a ser modificada sino hasta mucho después de la terminación del pulso. Estos mecanismos pueden ser diferentes según el material ablacionado, el líquido dentro del cual se encuentra sumergido el blanco y los parámetros de la fuente a utilizar como por ej. duración del pulso, longitud de onda, fluencia y frecuencia de los pulsos láser [75].

Las aplicaciones en el campo de los materiales se han incrementado con el desarrollo de sistemas de láser innovadores. Dentro de estos sistemas se encuentran los láseres de pulsos ultra cortos de alta intensidad, los que se han utilizado para procesamiento de materiales (micromaquinado) y en diversas aplicaciones como la deposición de películas delgadas de diversos compuestos sobre sustratos de vidrio o silicio. Una de las aplicaciones más estudiadas de los láseres de pulsos ultracortos en los últimos años es la fabricación de NPs metálicas. Algunos estudios realizados por Améndola et.al $76-78$ han proporcionado una descripción semi cuantitativa del proceso de ablación láser en interfaces sólido-líquido. Por otro lado, Amoruso et.al [79] estudiaron la distribución de tiempos de vuelo de iones positivos generados en el plasma producido por ablación láser sobre muestras sólidas de Al en vacío. En un trabajo posterior, Amoruso et.al [80] estudiaron la densidad de partículas generadas sobre un blanco de Al en vacío con diferentes fluencias, utilizando la técnica de espectroscopía de emisión óptica. Sin embargo, los mecanismos de formación no están completamente claros ya que se deben comprender con mayor profundidad los fenómenos de interacción del haz con el material a escala molecular.

El esquema experimental utilizado en el desarrollo de esta tesis para la síntesis de NPs por ablación láser de pulsos de fs en un medio líquido se muestra en Figura 3.1. 

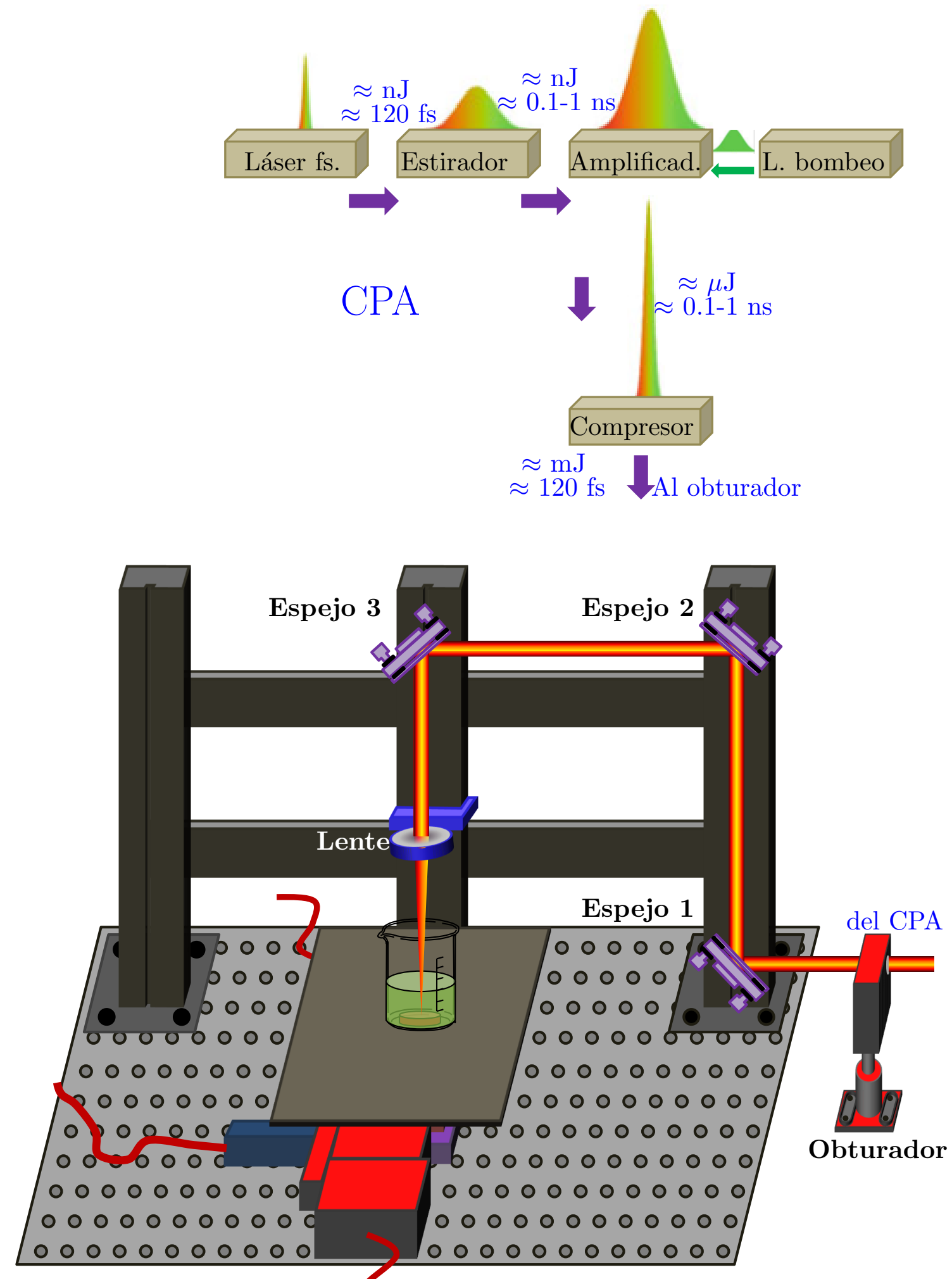

Figura 3.1: Esquema de amplificación de pulsos utilizando CPA y fabricación de NPs por ablación láser. 
El haz del láser es guiado por medio de tres espejos hacia la muestra, indicado en el esquema por una línea roja continua. Una lente de $5 \mathrm{~cm}$ de distancia focal asegura el enfoque del haz sobre la superficie sólida de metal colocada en la base de un vaso de precipitado, lleno de un medio líquido hasta cierta altura. Un sistema de posicionamiento de tres grados de libertad permite el desplazamiento de la muestra en en plano XY mientras que el eje $\mathrm{Z}$ permite un ajuste fino del enfoque.

Este sistema asegura que la ablación extraiga material en diferentes lugares de la superficie garantizando una fabricación estadísticamente homogénea. El obturador se utiliza para interrumpir el camino del haz láser cuando son necesarios cambios en la dirección de desplazamiento de la muestra.

En nuestro trabajo utilizamos un láser de Ti:Za de longitud de onda centrado en $800 \mathrm{~nm}$. Este láser, que cuenta con una etapa de amplificación de pulsos por barrido de frecuencia (CPA por sus siglas en inglés chirped pulse amplification), produce pulsos de 120 femtosegundos y su energía puede alcanzar los $1000 \mu \mathrm{J}$. La técnica CPA, como se muestra en la Figura 3.1 consiste en manipular temporalmente los pulsos de manera controlada y reversible para que la óptica involucrada no se vea afectada por picos de alta potencia 81 .

En este caso el sistema CPA consta de un oscilador, un ensanchador, un amplificador y un compresor. El oscilador consiste en un láser Ti:Za (mode-locked), que proporciona un tren de pulsos de fs con unos pocos nJ de energía a alta tasa de repetición (40 $\mathrm{MHz}$ ). Estos pulsos son ensanchados temporalmente (a ps-ns de duración) antes de la amplificación. El pulso amplificado se recomprime nuevamente a su duración original [82.

La duración de estos pulsos es más corta que los tiempos de transferencia de energía de electrones a iones, de conducción de calor y del tiempo hidrodinámico o de expansión. Debido a esto, el proceso de ablación láser ultrarápida básicamente no provoca daño térmico [83, que es la que trataremos de aquí en más.

Este tipo de ablación es descrita por Sivanandan et.al [81] como el resultado de varios procesos que ocurren durante y después de la interacción del pulso láser con el 
material a ablacionar. Cada uno de ellos posee una importancia propia en el proceso global de formación de nanopartículas.

Como se ilustra en la Figura 3.2 [81], para el caso de los metales, los electrones libres en la banda de conducción absorben la energía a través del proceso de Bremsstrahlung inverso (proceso que ocurre durante la duración del pulso). Luego de finalizar éste, para nuestro caso, entre los $120 \mathrm{fs}$ y 1ps, se produce una transferencia de energía de los electrones a la red cristalina (explosión Coulombiana). Luego de esta etapa se produce la vaporización térmica y la formación de la pluma de plasma que se encuentra acompañada de la formación de una onda acústica (onda de choque) propagándose en el líquido, seguida de una burbuja de cavitación [83, 84].

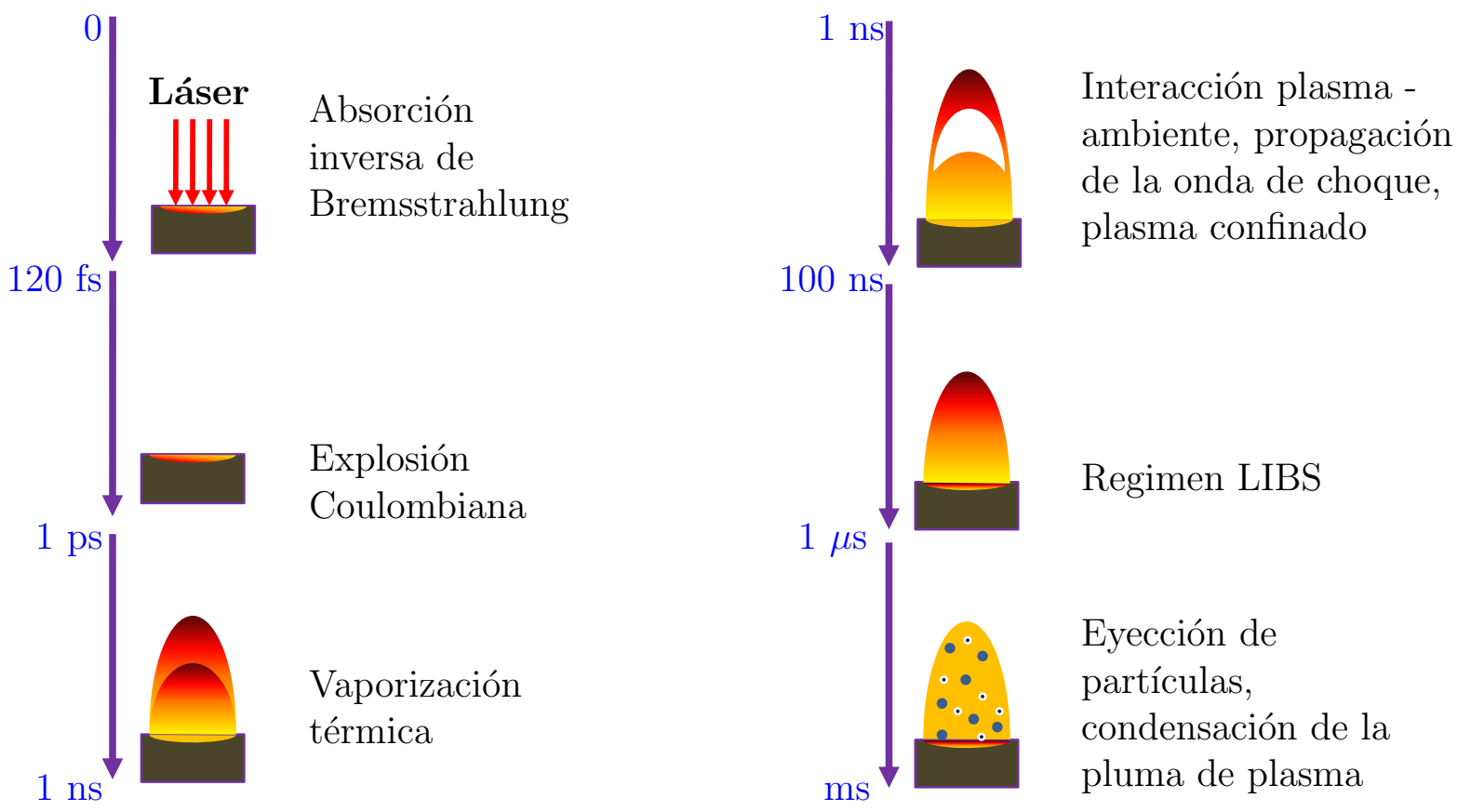

Figura 3.2: Resumen del proceso de ablación con un láser de fs (no térmica). (Adaptado de Perini et.al)

La duración de la pluma de plasma está en el rango de unos pocos nanosegundos a decenas de nanosegundos (dependiendo de la fluencia del láser y la duración del pulso), mientras que la duración de la onda de choque está en el rango de cientos de microsegundos [75]. La pluma de plasma posee la capacidad de ofrecer 
información espectroscópica de las especies en formación, habiéndose estudiado tanto teórica [85-88] como experimentalmente [89]. Luego, en tiempos cercanos a los ms se produce la eyección de partículas y la condensación de la pluma de plasma, como se ilustra en la Figura 3.3. Allí se muestra una amplificación del blanco sobre el que incide el haz y una representación de la pluma generada con los respectivos productos de la ablación: NPs y vapor.

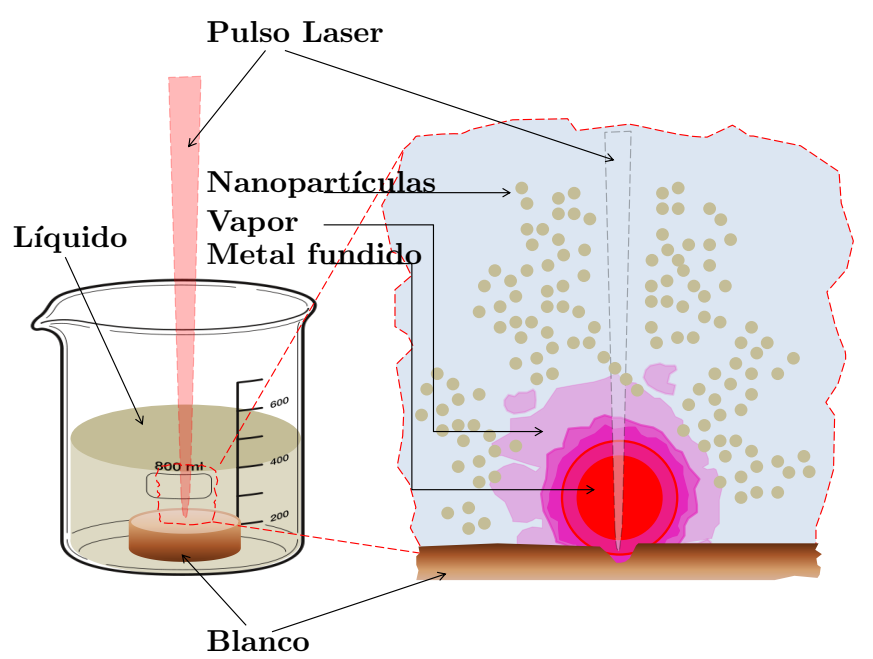

Figura 3.3: Ablación de un láser de femtosegundos sobre un blanco sumergido.

Para el caso específico del Al, las condiciones de producción de la pluma del plasma como también su duración han sido estudiadas por Drogoff et.al [90] y por Katsuya et.al [91]. En la Figura 3.4 se muestra la evolución de pluma formada durante el proceso de ablación sobre $\mathrm{Al}$ con un láser de 50 fs y una fluencia de $40 \mathrm{~J} / \mathrm{cm}^{2}[\mathbf{8 9}$, donde se observa el inicio cerca de los 100 ps y su colapso cerca de los 5 ms.

El fenómeno de ablación requiere una mínima fluencia para que ocurra, que está relacionada con la mínima energía que necesita el electrón para escapar del sólido [92]:

$$
F_{A}^{m}=\frac{3}{8}\left(E_{e}+E_{t}\right) \frac{c n_{e}}{\omega}
$$

donde $E_{e}$ es la energía de enlace atómico, $E_{t}$ es la función trabajo, $n_{e}$ es la densidad de electrones libres, $c$ velocidad de la luz y $\omega$ es la frecuencia del láser. Para el Al, la 
mínima fluencia para que ocurra ablación es $0.17 \mathrm{~J} / \mathrm{cm}^{2}[83]$.
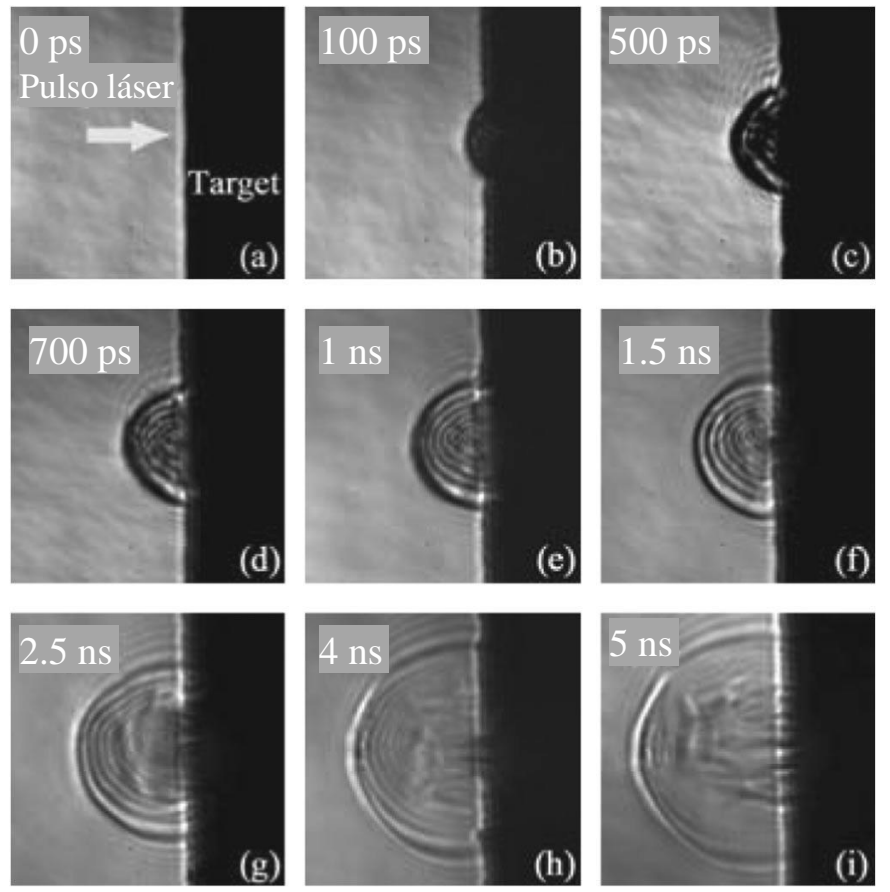

Figura 3.4: Fotografias de la extracción de material en los tiempos indicados para un blanco de Al. Utilizando un láser de 50 fs de duración de pulso y $40 \mathrm{~J} / \mathrm{cm}^{2}$ (extraido de Zhang et.al.

Para comprobar la relación entre la fluencia del láser con la profundidad de ablación Drogoff et.al [90] colocó Al de diferentes espesores sobre un sustrato de Si de $100 \mathrm{~nm}$. Su experimento consistió en producir ablación con un láser de fs y uno de ns sobre el dispositivo mencionado buscando la aparición de la línea característica del Si $288.16 \mathrm{~nm}$, como prueba de haber atravesado la capa de Al depositada sobre el sustrato. De esta manera, con una experiencia sencilla, podía estimar la fluencia umbral necesaria para ablacionar el Al. En la Figura 3.5 se muestra una adaptación gráfica de los resultados de este experimento. Ajustando los datos obtuvieron $\alpha^{-1}=232 \mathrm{~nm}$ y $\rho \Omega / \alpha=2.0 \mathrm{~J} / \mathrm{cm}^{2}$. Las mediciones se realizaron utilizando dos láseres diferentes Ti:Zafiro y Nd:YAG. En cuadrados rojos se muestra la masa removida por ablación láser en función de la energía suministrada al blanco de $\mathrm{Al}$. 


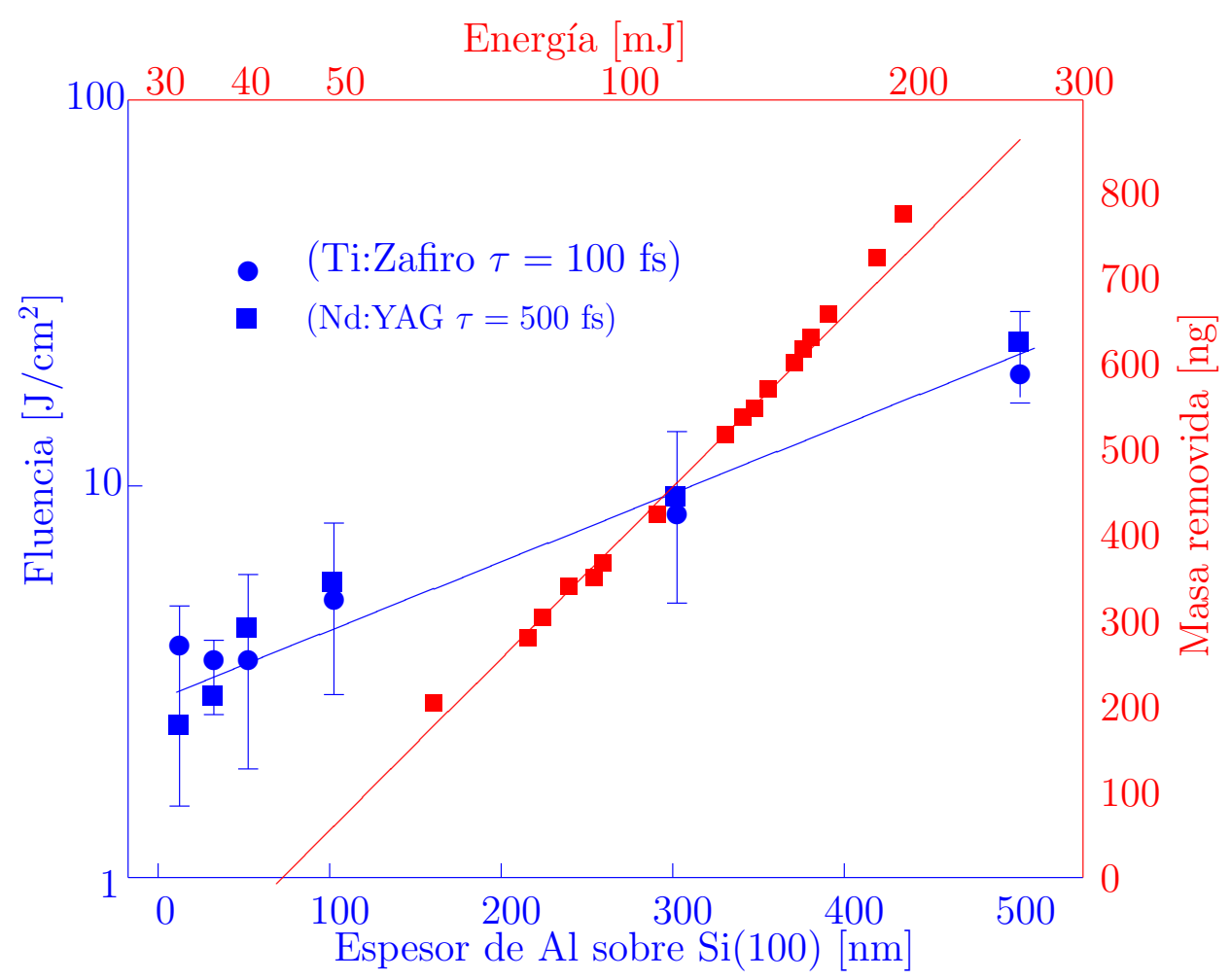

Figura 3.5: Umbral de fluencia para la aparición de la línea atómica de Si a 288.16 $\mathrm{nm}$ para seis diferentes capas de Al sobre un sustrato de Si de $100 \mathrm{~nm}$. (Adaptado de Drogoff et.al.)

Para la descripción de lo que ocurre durante el proceso de ablación láser, se suele utilizar el modelo de dos temperaturas, que supone que la termalización del subsistema de electrones es mucho mas rápida que la transferencia de energía a la red. Consideramos que los electrones adquieren una temperatura $T_{e}$ y que la red está caracterizada por una temperatura $T_{r}$. La descripción más sencilla consiste en modelar el sistema por un conjunto de dos ecuaciones diferenciales de difusión [85]:

$$
\begin{gathered}
c_{e} \frac{\partial T_{e}}{\partial t}=k_{e} \nabla^{2} T_{e}-\gamma\left(T_{e}-T_{r}\right) \\
c_{r} \frac{\partial T_{r}}{\partial t}=k_{r} \nabla^{2} T_{r}+\gamma\left(T_{e}-T_{r}\right),
\end{gathered}
$$

donde $c_{e} \quad \mathrm{y} \quad c_{r}$ son las capacidades caloríficas de los electrones y la red respectivamente. $k_{e}$ y $k_{r}$ son las conductividades caloríficas de los electrones y los fonones respectivamente. $\gamma=\pi^{2} m s^{2} N /\left(6 \tau_{0} T_{0}\right)$ es el coeficiente de transferencia de 
calor entre los electrones y la red [93. $T_{0}$ es la temperatura de Debye, $\tau_{0}$ es el tiempo de vuelo libre medio de los electrones a la temperatura $T_{0}, s$ es la velocidad del sonido, $N$ es el número de electrones libres por unidad de volumen y $m$ es la masa electrónica.

Sintetizando, en este Capítulo 3 se realiza una descripción del método de generación de NPs por ablación láser de pulsos ultracortos, como así también el modelo estándar sobre los mecanismos de síntesis de NPs por este método. 


\section{Capítulo 4}

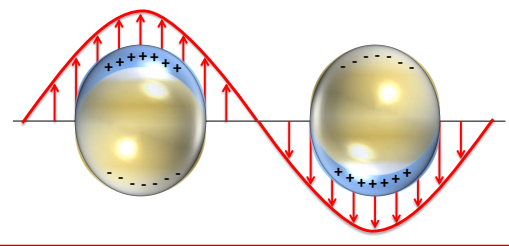

Caracterización de nanopartículas por espectroscopía de extinción óptica

Si caminas solo

irás más rápido;

si caminas acompañado

llegarás más lejos

Proverbio chino.

A medida que avanzan las capacidades de síntesis de NPs, existe una creciente necesidad de un análisis fiable para determinar la distribución del tamaño de las mismas y su estructura. Existen varias técnicas para detectar, medir y caracterizar NPs aunque ninguna de ellas pueda ser considerada como la "mejor". Todas presentan ventajas y desventajas dependiendo del tipo de muestra y del principio en el cual se basan.

Las más tradicionales son aquéllas que se basan en la interacción de electrones con la muestra a medir, como la microscopía electrónica, tanto de transmisión (TEM) como de barrido (SEM). La primera se puede utilizar para obtener una imagen de las NPs (a tamaños que se aproximan a la escala atómica) por medio de un haz de electrones que atraviesa la muestra, determinando tamaño, estructura y composición de la muestra. Si bien posee la ventaja de poder formar una imagen directa de las NPs, muestra desventajas como la de tener una estadística relativamente baja, la necesidad de una determinación conveniente del límite de NP-fondo y el posible daño del haz de electrones sobre las NPs. 
El segundo tipo de microscopía (SEM) se basa en la detección de electrones retrodispersados por la muestra. Para lograr un buen contraste es necesario que la misma sea metálica o que posea una fina capa conductora (sputtering de oro o paladio). Esta microscopía puede determinar tamaño y composición, pero no alcanza la escala atómica ni a determinar la estructura de las NPs. Comparte con TEM las desventajas comparativas de necesitar una cuidadosa preparación de la muestra, trabajar con alto vacío, presentar baja estadística de muestreo y un alto costo de inversión.

Otra técnica muy utilizada dentro de las microscopías, es la llamada de fuerza atómica (AFM). Se basa en el sensado de las fuerzas de atracción entre la muestra y una punta muy fina (sonda nanoscópica) sostenida por una estructura flexible (cantilever). La deflexión mecánica de este cantilever se traduce en la deflexión óptica de un haz láser incidente sobre él, siendo ésta registrada por detectores de cuadrante apropiados. Este tipo de microscopía permite estudiar la morfología de NPs de hasta $1 \mathrm{~nm}$ de tamaño, admitiendo una visualización 3D. La muestra debe prepararse en forma cuidadosa sobre sustratos de alta planitud (mica), y, aunque no trabaja en vacío, no permite estudiar ni la estructura ni la composición. No posee una buena estadística de muestreo ya que las suspensiones de NPs deben presentarse con una alta dilución.

Por otro lado, existen técnicas ópticas que se basan en el scattering y/o absorción de luz por parte de suspensiones de NPs. Una de las más comunes es la llamada scattering dinámico de luz (Dynamic Light Scattering, DLS), en la que a través de la técnica de correlación de fotones se detecta (con resolución temporal) el scattering de un haz láser que incide sobre la muestra macroscópica, utilizando un fotomultiplicador ubicado a $90^{\circ}$ respecto de la dirección de incidencia. A partir de la correlación de las mediciones es posible determinar el tamaño promedio y la distribución de tamaños. El tamaño obtenido corresponde al diámetro hidrodinámico de la NP. Para obtener el tamaño verdadero debe tenerse en cuenta la viscosidad del medio. 
Cuando el tamaño de las NPs disminuye a unas pocas decenas de nm, el scattering resulta ser casi nulo comparado con la absorción. Para el caso de NPs metálicas, ésta se ve realzada por la llamada resonancia plasmónica que, en el caso de metales nobles $(\mathrm{Au}, \mathrm{Ag}, \mathrm{Cu})$, aparece como una banda de absorción en la zona del espectro visible, que está relacionada con parámetros característicos de la muestra, como por ejemplo el tipo y tamaño del material particulado, su índice de refracción relativo al medio entre otros. Por esta razón, la espectroscopia de extinción óptica resulta ser un método competitivo a la hora de determinar distribución de tamaño, estructura y composición. Además, permite el análisis de muestras in situ y en estado nativo. En la Figura 4.1 se muestra un esquema de los procesos de scattering y absorción y su relación con el concepto de extinción óptica.

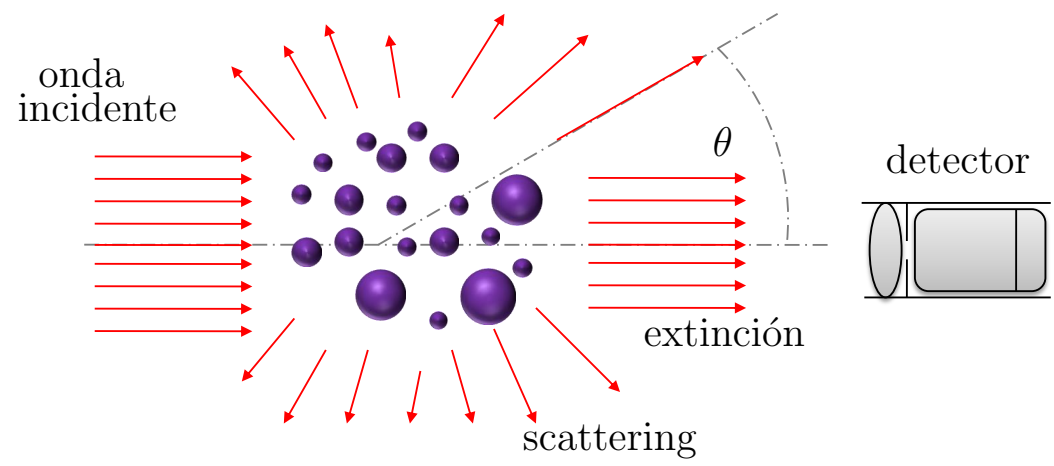

Figura 4.1: Esquema de los procesos de scattering y extinción de un conjunto de partículas.

Un espectrofotómetro mide la extinción de luz, que es la fracción de la luz del haz incidente que no llega al detector. Operativamente, la extinción puede ser derivada de la relación dada por la ley de Lamber-Beer:

$$
I_{t}=I_{i} e^{-\beta l}
$$

donde $I_{i}$ e $I_{t}$ son las intensidades incidente y transmitida respectivamente, $\beta$ es el coeficiente de atenuación y $l$ es la longitud de la muestra. Cuando tenemos una 
dilución tal que permite considerar que la distancia entre NPs es grande respecto de la longitud de onda, el scattering total es la suma del scattering por partícula (condición llamada de scattering simple). En estas condiciones, es posible relacionar el coeficiente de atenuación $\beta$ con la sección eficaz de extinción de partícula aislada $C_{\text {ext }}$ (que depende del tamaño) por medio del número de NPs por unidad de volumen $N$. Los llamados espectros de extinción se obtienen de mediciones de $I_{t} / I_{i}$ para distintos valores de longitud de onda. Estos resultados experimentales pueden ser reproducidos teóricamente, utilizando el tamaño como parámetro de ajuste.

Como resultado de la fabricación de NPs metálicas en medios líquidos, tanto por método físicos como por métodos químicos, los coloides obtenidos no resultan ser monodispersos, sino que presentan una distribución de tamaños. En este caso, es esta distribución la que debe utilizarse como parámetro de ajuste. Dicha distribución está usualmente representada por una función log-normal, cuya expresión está definida por la ecuación:

$$
f(R)=\frac{1}{R \sigma_{d i s} \sqrt{2 \pi}} \exp \left(-\frac{\left(\ln R-\mu_{d i s}\right)^{2}}{2 \sigma_{d i s}^{2}}\right),
$$

donde $R$ es el radio de las NPs. El radio con mayor probabilidad (donde la función $f(R)$ alcanza su máximo valor) está definido en términos de los parámetros de la distribución $\sigma_{d i s}$ y $\mu_{d i s}$, como $R_{m}=\exp \left(\mu_{d i s}-\sigma_{d i s}^{2}\right)$. El radio medio $\bar{R}$ y el coeficiente de varianza $C$ de las NPs que conforman la distribución de tamaños de NPs se definen como $\bar{R}=\exp \left(\mu_{d i s}-0,5 \sigma_{d i s}^{2}\right)$ y $C=\sqrt{\exp \left(\sigma_{d i s}^{2}\right)-1}$. Otro parámetro utilizado para definir una distribución son el radio efectivo $R_{e f}=\exp \left(\mu_{d i s}+2,5 \sigma_{d i s}^{2}\right)$ y la varianza efectiva $v_{e f}=\exp \left(\mu_{\text {dis }}\right)-1$.

Para considerar la distribución de tamaños en las muestras, se debe discretizar la función $f(R)$ para todo el rango de valores donde $f(R) \neq 0$ y determinar la extinción utilizando la teoría de Mie para cada uno de los radios. Para obtener la extinción total de la muestra, se deben sumar cada una de las contribuciones para lo cual estas se deben multiplicar por sus respectivos valores $f(R)$. 


\subsection{Caracterización de nanopartículas de Au.}

Los métodos de fabricación tienden a sintetizar NPs metálicas de geometría esférica en configuraciones simples o complejas. Las primeras presentan un núcleo descubierto (núcleo desnudo), mientras que las segundas presentan una o más coberturas de diferente material que el núcleo (núcleo-cubierta). Como ejemplos de aplicación de la técnica de extinción óptica para la determinación de distribución de tamaños, estudiaremos algunos casos realizados durante esta tesis, comenzando por NPs de núcleo desnudo de oro y de magnetita y continuando con NPs núcleo-cubierta de $\mathrm{Al}_{-} \mathrm{Al}_{2} \mathrm{O}_{3}$, Aire- $\mathrm{Al}_{2} \mathrm{O}_{3}$, Aire- $\mathrm{Al}$ y $\mathrm{Fe}_{3} \mathrm{O}_{4}-\mathrm{Au}$.

En la Figura 4.2 se muestra el espectro de extinción normalizado (círculos grises) de NPs de Au fabricadas por un grupo colaborador, utilizando el método de micela inversa en n-heptano. Para la obtención de los espectros de extinción teoricos, se utilizó la función dieléctrica corregida por tamaño y una distribución log-normal para los tamaños de las NPs. La curva que muestra mejor concordancia entre los valores experimentales y el modelo teórico corresponde al ilustrado en línea negra. Los parámetros de la función log-normal que describe este espectro de extinción son $R_{m}=0.8$ y $\sigma=0.4$. En azul se muestra el espectro de extinción en caso de variar el valor de $R_{m}$ a 0.9 y en rojo al variar $\sigma$ a 0.5 . Estos dos espectros muestran un ajuste de mayor error que el correspondiente a la línea negra. Lo anterior implica que se pueden ajustar espectros con buena coincidencia.

Se realizó en forma independiente un análisis TEM de la misma muestra. Los resultados se grafican en barras grises de contorno rojo en la Figura 4.2 y en segmentos azules su respectiva curva log-normal. Se observa una discrepancia entre los valores obtenidos utilizando espectroscopía de extinción y TEM. La principal variable que influye en esta diferencia está relacionada con la cantidad de NPs observadas en cada

una de las técnicas. La espectroscopia de extinción permite observar alrededor de $10^{12}$ NPs, mientras que la técnica TEM toma como muestra cientos de NPs (en este caso el histograma TEM corresponde a cerca de 400 NPs). 


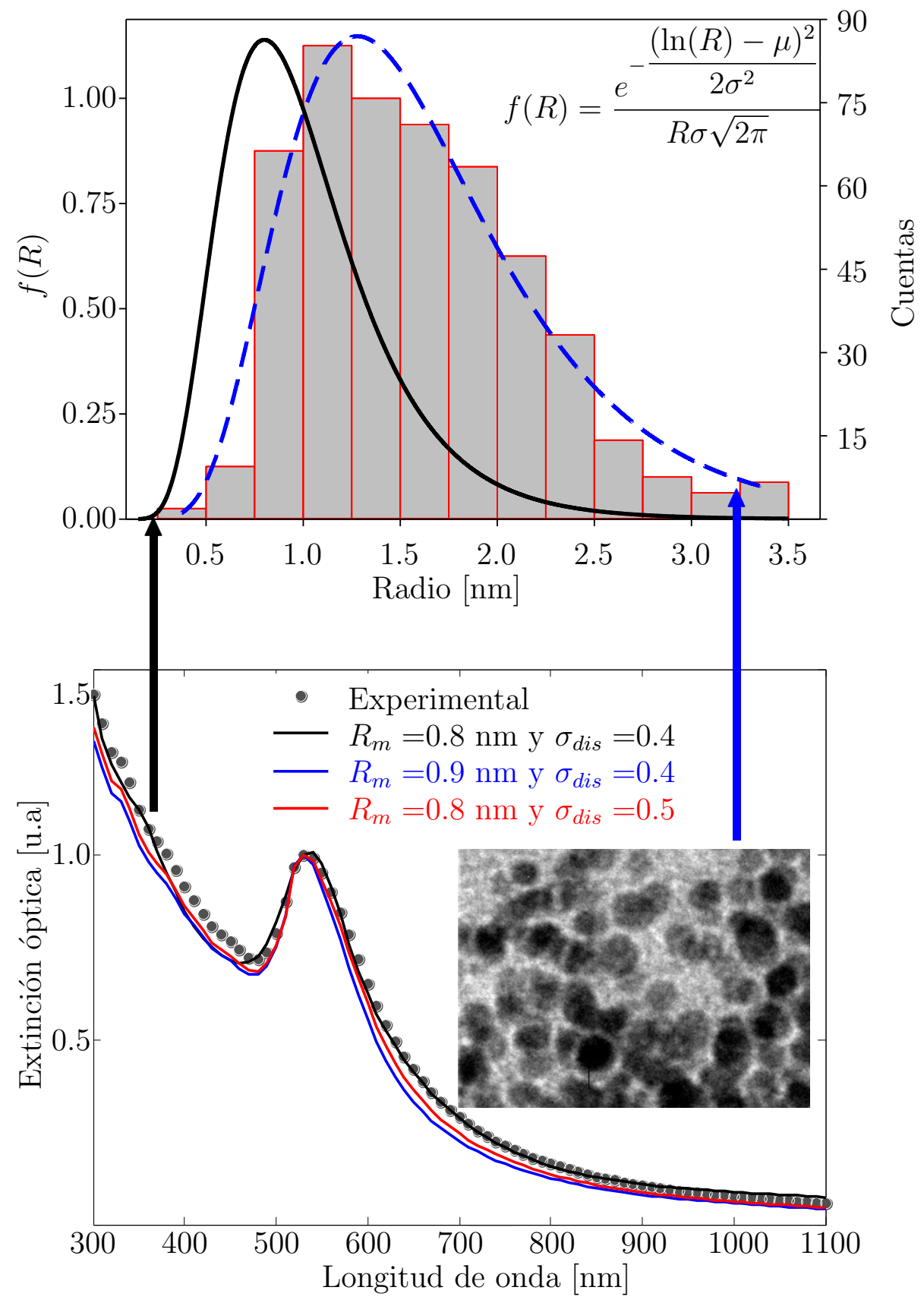

Figura 4.2: Ajuste del espectro experimental de NPs de Au (en puntos grises), en línea negra para $R_{m}=0.8 \mathrm{~nm}$ y $\sigma_{d i s}=0.4$, que es el espectro teórico que mejor ajusta, en línea roja para $R_{m}=0.8 \mathrm{~nm}$ y $\sigma_{d i s}=0.5$ y en línea azul para $R_{m}=0.9 \mathrm{~nm}$ y $\sigma_{d i s}=0.4$. 


\subsection{Caracterización de nanopartículas de magnetita.}

Otro ejemplo de caracterización de NPs de núcleo desnudo por espectroscopia de extinción tratado en esta tesis corresponde a NPs de magnetita $\left(\mathrm{Fe}_{3} \mathrm{O}_{4}\right)$. Cabe aclarar que las muestras coloidales de magnetita en agua y n-heptano, fueron obtenidas por el Dr Ignacio Bruvera utilizando síntesis química, durante sus estudios doctorales realizados en el grupo de magnetismo del Instituto de Física de La Plata (IFLP). Cuando las NPs que se desean describir presentan propiedades magnéticas importantes (como en el caso de la magnetita), se debe tener la precaución de conocer la región del espectro en las que éstas tienen influencia. En el Apéndice B, se describe la determinación de la permeabilidad magnética para el caso de la magnetita, utilizando la ecuación de Gilbert. También se muestra allí que en la región óptica se puede considerar la permeabilidad magnética como $\mu=1$.

El espectro de extinción experimental de NPs de magnetita en agua se muestra en línea continua roja en el panel inferior de la Figura 4.3 , junto con el ajuste teórico (en líne de segmentos azules) y una fotografía TEM de parte de la muestra. En el panel superior se muestra el histograma TEM con su respectiva curva log-normal (línea continua negra) y en segmentos azules la distribución de tamaños obtenida por espectroscopía de extinción. El radio mas probable en el caso de la técnica TEM es 4.9 $\mathrm{nm}$, mientras que el ajuste del espectro con teoría de Mie arroja un valor de $5.3 \mathrm{~nm}$. Los respectivos valores de $\sigma$ para cada una de las distribuciones son 0.14 y 0.15 para las dos técnicas respectivamente. 


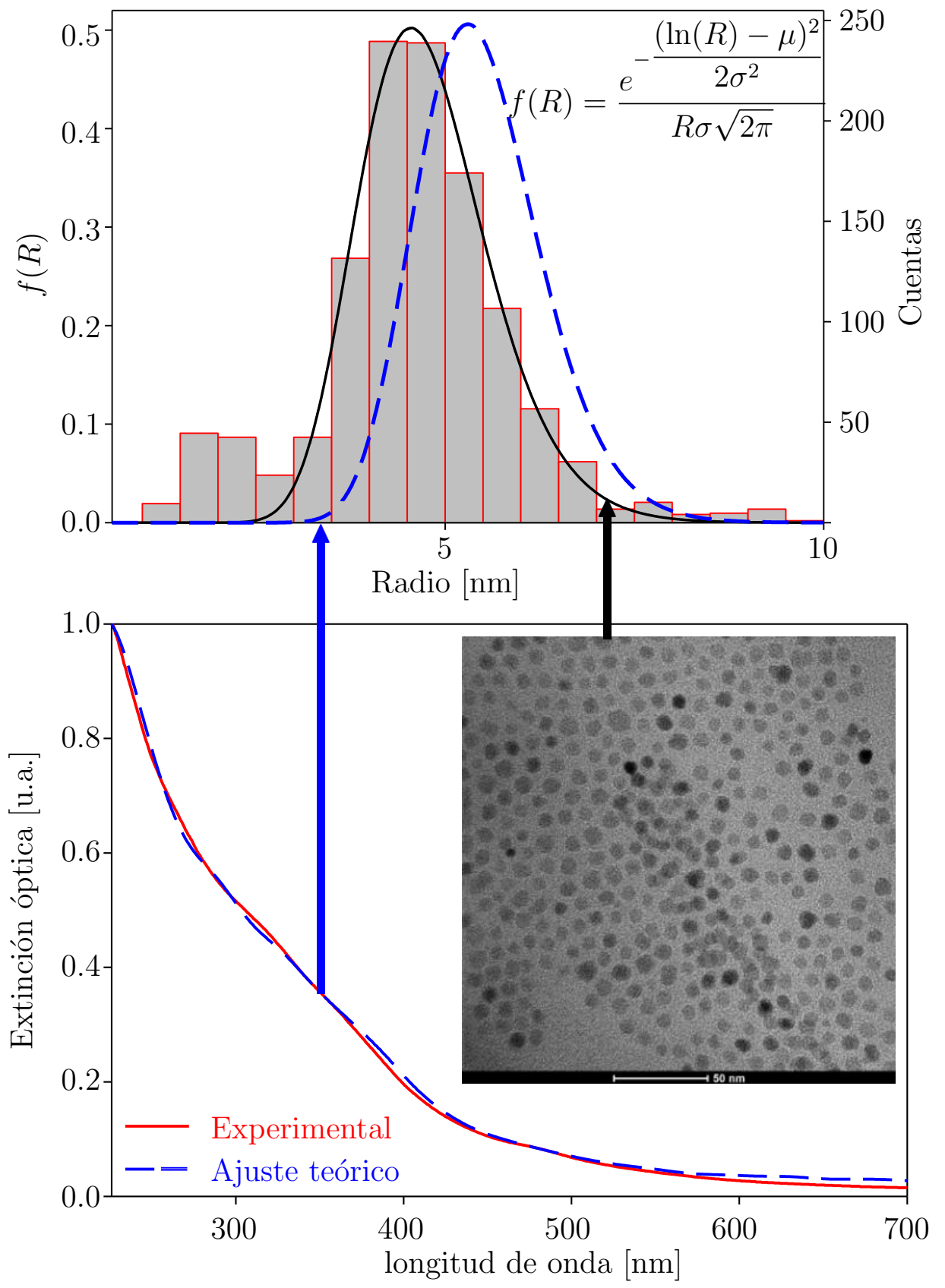

Figura 4.3: Espectro experimental y teórico de NPs de magnetita en agua (panel inferior). Distribución de tamaño determinada por espectroscopía óptica (línes de segmentos azul) e histograma TEM con curva log-normal (panel superior).

De forma similar al caso anterior, se muestra en la Figura 4.4 los resultados para las muestras de NPs de magnetita suspendidas en n-heptano: en línea roja el espectro experimental de NPs magnetita y en segmentos azules el ajuste teórico con la respectiva distribución de tamaños. 


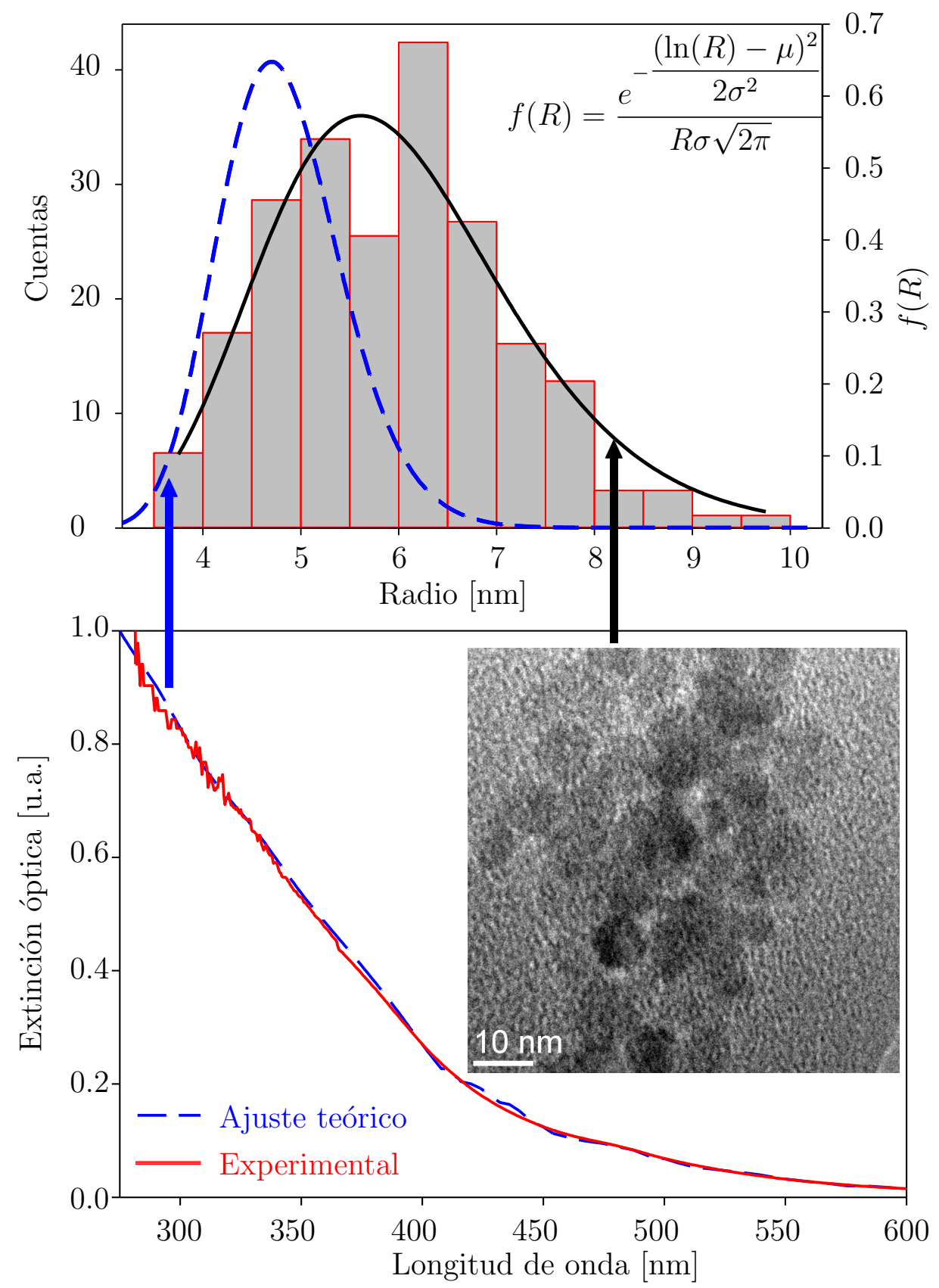

Figura 4.4: Espectro experimental y teórico de NPs de magnetita en n-heptano (panel inferior). Distribución de tamaño determinada por espectroscopía óptica (línes de segmentos azul) e histograma TEM con curva log-normal (panel superior).

En este caso el radio mas probable en el caso de la técnica TEM es $5.35 \mathrm{~nm}$, mientras que la espectroscopía óptica en conjunto con teoría de Mie arrojan 4.7 nm. Los respectivos valores de $\sigma$ para cada una de las distribuciones son 0.14 y 0.15 para las dos técnicas respectivamente. En la parte superior de la Figura 4.4, se muestra 
en barras grises con contorno rojo el histograma obtenido por la técnica TEM con su respectiva curva log-normal (línea continua negra) y en segmentos azules la distribución de tamaños obtenida por espectroscopia de extinción.

Es importante recordar que cuando se utiliza la espectroscopía óptica de extinción para caracterizar NPs, la porción de muestra iluminada por el espectrofótometro cubre un número significativamente superior de NPs que la técnica de microscopia electrónica de barrido (TEM). Lo antes expuesto explica las diferencias entre las distribuciones de tamaño obtenidas por cada una de las técnicas de caracterización.

\subsection{Caracterización de nanopartículas núcleo-cubierta de $\mathrm{Fe}_{3} \mathrm{O}_{4}$-Au.}

En la sección anterior determinamos la distribución de tamaños de NPs de núcleo desnudo de $\mathrm{Au} \mathrm{y} \mathrm{Fe}_{3} \mathrm{O}_{4}$, en las cuales los parámetros a determinar corresponde a $R_{m}$ y $\sigma$. Cuando las NPs presentan una cubierta, los parámetros que caracterizan la distribución de tamaños corresponden son $R_{1}, R_{2}$ у $\sigma$.

Para caracterizar NPs núcleo-cubierta de la forma $\mathrm{Fe}_{3} \mathrm{O}_{4}-\mathrm{Au}$, es importante tener en cuenta que la cubierta de Au produce un pico plasmónico en la región visible. Este pico plasmónico puede ser utilizado para la caracterización por tamaño de las NPs. Debido al cambio de la extinción de las NPs con núcleo de magnetita y cubierta de Au con los tamaños del núcleo y cubierta, es de esperar que cuando se tienen distribuciones de tamaño (como ocurre usualmente durante la fabricación de estas NPs [94-98]) la extinción dependa de la distribución de tamaños de las NPs. Cuando la distribución de tamaños es mas ancha el pico plasmónico se ubica en longitudes de onda mas largas.

Si las NPs sintetizadas poseen una distribución de tamaños muy angosta $(\sigma \approx 0$ monodispersión), se reducen los parámetros a determinar $\left(R_{1}\right.$ y $\left.R_{2}\right)$. En este caso, se ha desarrollado en esta tesis un método paramétrico que consiste en graficar la ubicación del pico plasmónico $\left(\lambda_{p}\right)$ y la longitud de onda altura del $70 \%$ de la amplitud del pico plasmónico $\left(\lambda_{s}\right)$ para diferentes radios y diferentes espesores de cubierta (se utiliza $70 \%$ y no $50 \%$ como es usual debido a que la mayor parte de los espectros de estas NPs son 
anchos y no simétricos, de modo que no se alcanza el $50 \%$ en el rango de longitudes de onda estudiado). Esto se muestra en la Figura 4.5 para $\mathrm{NPs}_{\mathrm{s}} \mathrm{Fe}_{3} \mathrm{O}_{4}$ - $\mathrm{Au}$. Utilizando los valores experimentales de $\lambda_{p} \mathrm{y} \lambda_{s}$, se grafican curvas de nivel y el cruce de las misma corresponde a la solución única buscada.

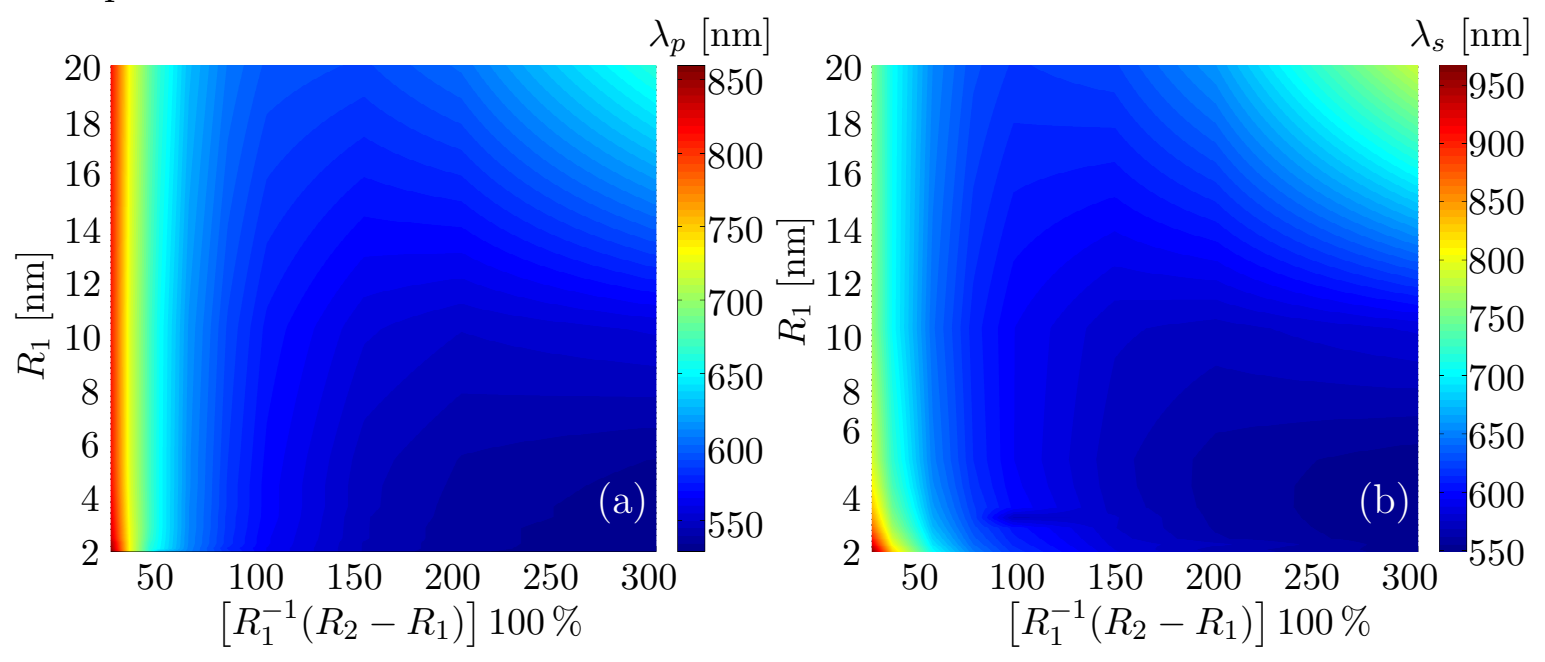

Figura 4.5: (a) Gráfico de la longitud de onda del pico plasmónico para el radio de la magnetita variable y el porcentaje de la cubierta con respecto al núcleo. (b) Gráfico de la longitud de onda a la altura $70 \%$ del pico plasmónico para el radio de la magnetita variable y el porcentaje de la cubierta con respecto al núcleo.

Como ejemplo de aplicación de este método paramétrico, se pueden tomar los resultados de Lingyan et al [99], quienes han fabricado $\mathrm{NPs}$ de $\mathrm{Fe}_{3} \mathrm{O}_{4}$ - $\mathrm{Au}$ a partir de NPs de $\mathrm{Fe}_{3} \mathrm{O}_{4}$ de dos tamaños monodispersos seleccionados. Los espectros obtenidos de estas NPs en agua muestran valores de $\lambda_{p}$ de $558 \mathrm{~nm}$ y $583 \mathrm{~nm}$ y valores de $\lambda_{s}$ de $593 \mathrm{~nm}$ y $623 \mathrm{~nm}$ respectivamente.

Tomando curvas de nivel para estos valores en las Figuras 4.5 y las graficamos en el mismo plano, el punto donde se cortan estas dos gráficas determina el radio del núcleo y el porcentaje del espesor de la nanocubierta. En la Figura 4.6, se muestran la curva de nivel correspondientes a $\lambda_{p}=558 \mathrm{~nm}$ y $\lambda_{s}=593 \mathrm{~nm}$ en negro y rojo respectivamente, el corte entre estas dos curvas de nivel muestra que $R_{1}=[2,14 \pm 0,4]$ nm y $R_{2}=[4,75 \pm 0,6] \mathrm{nm}$, valores obtenidos por métodos paramétricos. Adiconalmente, se muestran la curva de nivel correspondientes a $\lambda_{p}=583 \mathrm{~nm}$ y $\lambda_{s}=623 \mathrm{~nm}$ en azul y verde respectivamente, el corte entre estas dos curvas de nivel muestra que 
$R_{1}=[2,70 \pm 0,4] \mathrm{nm}$ y $R_{2}=[4,93 \pm 0,7] \mathrm{nm}$.

En cada caso se muestran dos curvas de nivel adicionales en líneas segmentadas (1 $\mathrm{nm}$ por encima y $1 \mathrm{~nm}$ por debajo) para $\lambda_{p} \mathrm{y} \lambda_{s}$ respectivamente con la finalidad de ilustrar la resolución del método. Los valores de los radios para las muestras obtenidas por TEM en el primer caso son $R_{1}=2,25 \mathrm{~nm}$ y $R_{2}=3,40 \mathrm{~nm}$. Para el segundo caso por el mismo método $R_{1}=2,70 \mathrm{~nm}$ y $R_{2}=3,50 \mathrm{~nm}$. Las diferencias observadas entre los tamaños determinados por los dos métodos puede ser atribuida a dos razones. Por un lado, la menor estadística registrada por la técnica TEM comparada con la que brinda espectroscopía de extinción y por otro, el error experimental en la medida de la longitud de onda utilizada en la técnica paramétrica.

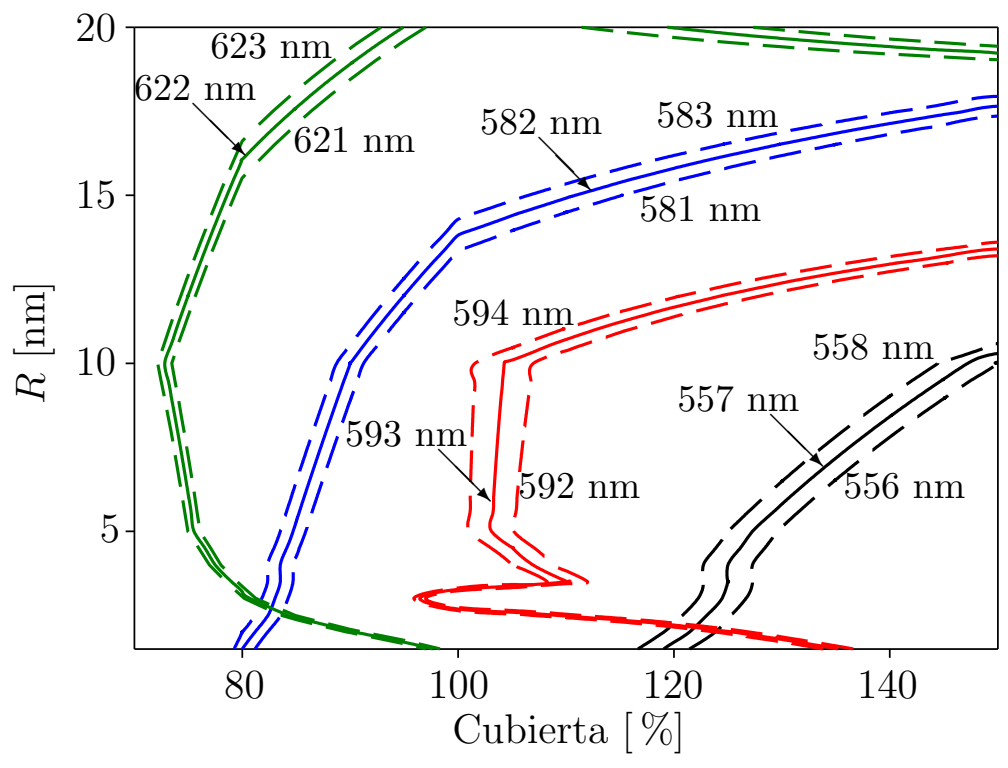

Figura 4.6: Curvas de nivel del pico y ancho del pico plasmónico para los espectros experimentales de NPs de $\mathrm{Fe}_{3} \mathrm{O}_{4}$-Au dadas en Lingyan et al.

Cuando las NPs presentan una dispersión de tamaños, se puede emplear este modelo de caracterización en el caso de NPs fabricadas por métodos químicos, dado que en este caso es usual obtener primero NPs de núcleo desnudo y luego formar la cubierta de estas NPs. En este caso se toman dos espectros, uno correspondiente a las NPs de núcleo desnudo y otro correspondiente a las NPs con las cubierta. Con el primero de estos se determinan los valores de $R_{1}$ y $\sigma$ y con el segundo de estos espectros de 
extinción se determina $R_{2}$ y en el caso de desconocer el índice de refracción de la cubierta también es posible determinarlo. En el caso de NPs obtenidas por ablación láser se deben determinar simultaneamente los parámetros, el método para determinar la distribución de tamaños $\left(R_{1}, R_{2}\right.$ y $\left.\sigma\right)$, se minimiza el error entre el espectro experimental y las predicciones teóricas hasta obtener los valores mas adecuados de $R_{1}, R_{2}$ y $\sigma$.

\subsection{Caracterización de nanopartículas de $\mathrm{Al}$ fabricadas por ablación láser}

Utilizando el esquema de ablación láser descrito en el Capítulo 3, se fabricaron NPs a partir de un blanco de Al, sumergido en n-heptano y en agua.

En la Figura 4.7, se observa un espectro de extinción de NPs de Al fabricadas por ablación láser a partir de un blanco de Al sumergido en n-heptano utilizando una energía del pulso de fs incidente de $500 \mu \mathrm{J}$.

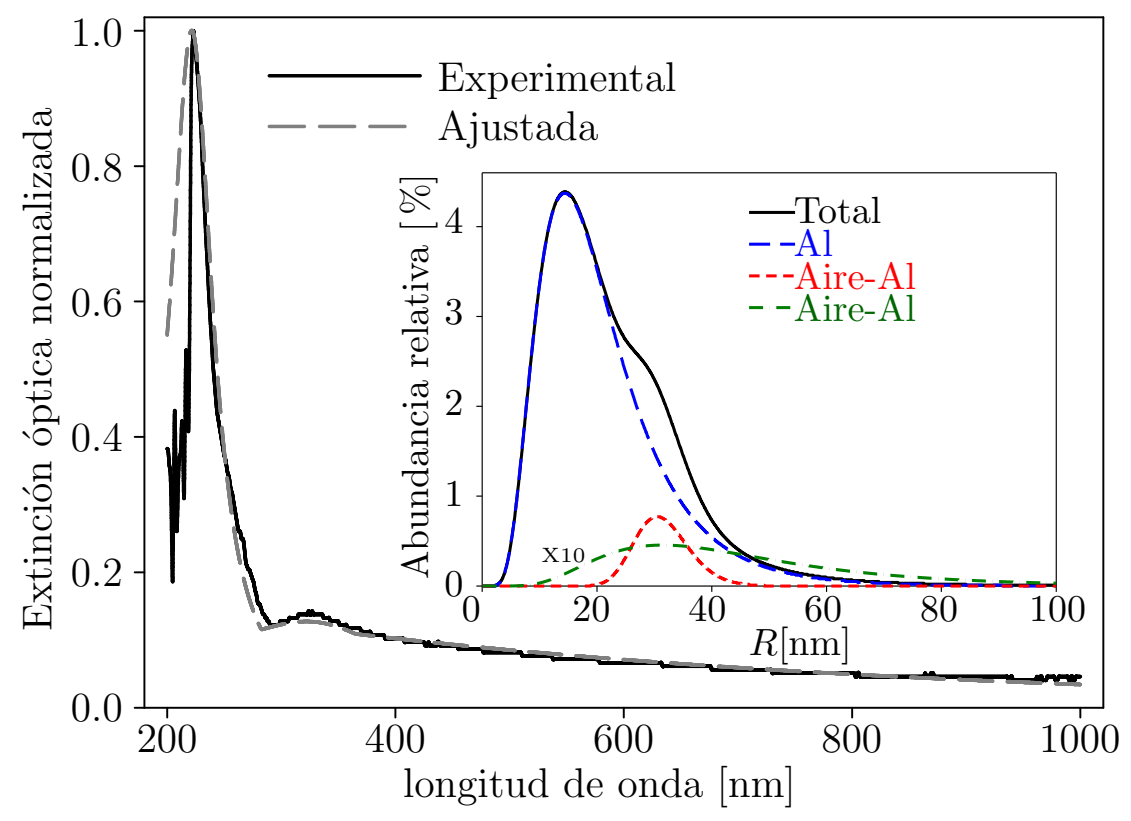

Figura 4.7: Espectro experimental de NPs de Al fabricadas por ablación láser en n-heptano, con su respectivo ajuste y distribución de tamaños.

El espectro de estas NPs se ajustó con funciones log-normal para cada una de las especies posibles en el medio (Al, aire-Al). En este caso debemos determinar para las 
NPs de Al los valores de $R_{m}$ y $\sigma$ y para las NPs de aire-Al el radio del núcleo $R_{m}, \sigma$ y el porcentaje de la cubierta. Adicionalmente se deben determinar los porcentajes de cada una de estas especies en la muestra.

Las distribuciones de tamaño obtenidas se muestran en el recuadro de la Figura 4.7. Para la especie Aire-Al, se muestran dos distribuciones que corresponden a porcentajes diferentes de espesor de la cubierta. Los parámetros de las distribuciones de tamaño obtenidas se resumen en la Tabla 4.1 .

Aplicando el mismo procedimiento para el ajuste de NPs de Al fabricadas en n-heptano, se ajustó el espectro de extinción de NPs de Al fabricadas en agua con una energía del pulso de fs incidente de $500 \mu \mathrm{J}$. Notar que, para este caso, el espectro no presenta pico plamónico lo que es indicativo de la posible existencia de NPs de óxido de $\mathrm{Al}$ como así tambien de NPs de $\mathrm{Al}$ recubiertas de óxido.

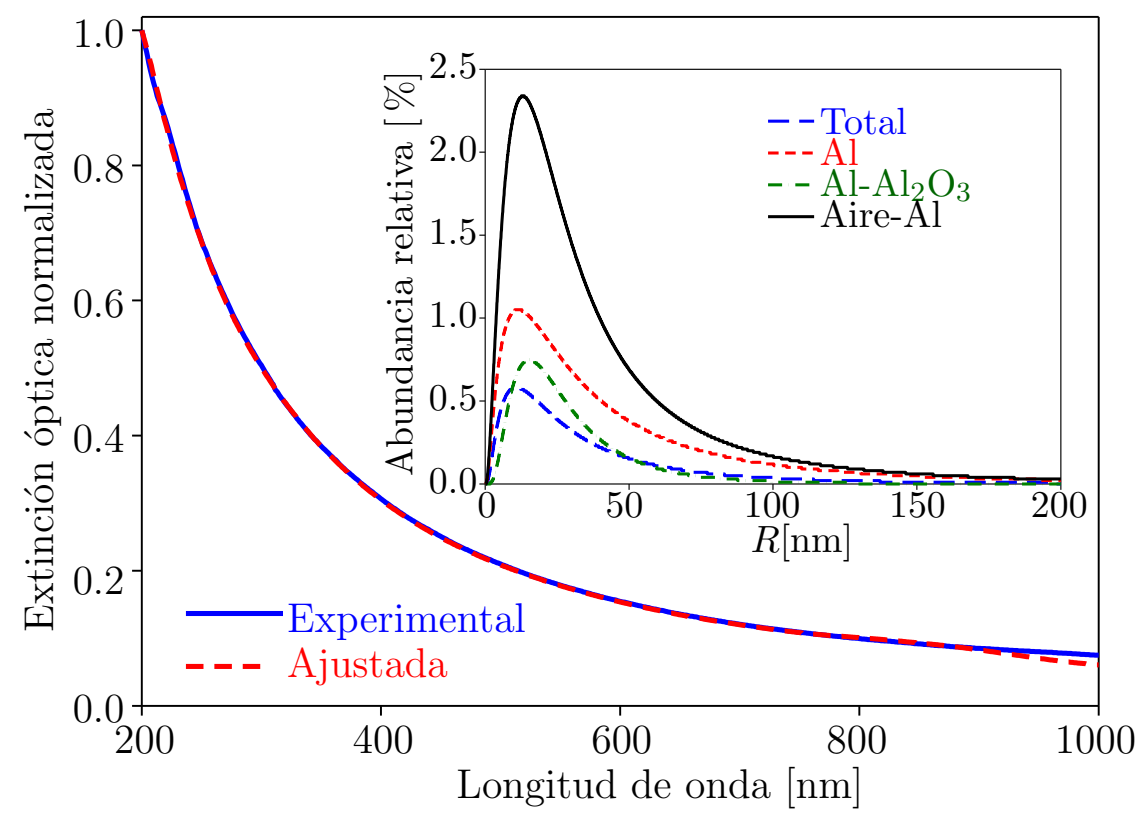

Figura 4.8: Espectro experimental de NPs de Al fabricadas por ablación láser en agua, con su respectivo ajuste y distribución de tamaños.

En este caso, se deben considerar adicionalmente entonces las especies $\mathrm{Al}_{2} \mathrm{O}_{3}$, Al- $\mathrm{Al}_{2} \mathrm{O}_{3}, \mathrm{Al}_{2} \mathrm{O}_{3}-\mathrm{Al}$ y Aire- $\mathrm{Al}_{2} \mathrm{O}_{3}$. El espectro ajustado y sus respectivas distribuciones de tamaño se muestran en la Figura 4.8. Los parámetros de las distribuciones de tamaño presentes se resumen también en la Tabla 4.1 . 


\begin{tabular}{|c|c|c|c|c|c|}
\hline Al & Especies & $R_{m}[\mathbf{n m}]$ & $\sigma$ & cubierta $(\%$ de $a)$ & Porcentaje \\
\hline \multirow{4}{*}{$\frac{\stackrel{\vec{d}}{0}}{\frac{d}{d}}$} & Al & 14.4 & 0.54 & 0 & 89 \\
\hline & Aire-Al & 29.8 & 0.15 & 11 & 9 \\
\hline & Aire-Al & 30.6 & 0.52 & 21 & 2 \\
\hline & Especies & $R_{m}[\mathbf{n m}]$ & $\sigma$ & cubierta $(\%$ de $a)$ & Porcentaje \\
\hline \multirow{3}{*}{$\begin{array}{l}\frac{\sigma}{2} \\
\frac{\infty}{4}\end{array}$} & $\mathrm{Al}$ & 10.8 & 0.94 & 0 & 23 \\
\hline & $\mathrm{Al}-\mathrm{Al}_{2} \mathrm{O}_{3}$ & 11.3 & 1.03 & 19.4 & 53 \\
\hline & Aire-Al & 15.1 & 0.67 & 20.3 & 24 \\
\hline
\end{tabular}

Tabla 4.1: Especies de NPs presentes en la solución coloidal de Al en n-heptano y agua fabricadas por ablación láser

Un análisis independiente realizado por AFM, muestra resultados muy similares a los obtenidos por espectroscopia de extinción, recordando que la estadística de muestras de NPs en la técnica AFM es muy inferior y que no permite identificar las especies presentes.

La Figura 4.9 (a) muestra la imagen de un cuadro de área $40 \mu \mathrm{m} \times 20 \mu \mathrm{m}$ donde se observan diferentes NPs. Con la finalidad de observar en el medio NPs mas pequeñas se reduce el área a $14 \mu \mathrm{m} \times 14 \mu \mathrm{m}$ Figura 4.9 (b), donde se observan NPs de radios entre 20 nm y 40 nm. Finalmente reduciendo mucho más el área de observación $3 \mu \mathrm{m} \times$ $2 \mu \mathrm{m}$ Figura 4.9 (c), se aprecian NPs entre $4 \mathrm{~nm}$ y $15 \mathrm{~nm}$. En las subfiguras (d)-(f) se muestran perfiles de algunas líneas seleccionadas, donde se muestran ejemplos de tamaños presentes. En la Figura 4.9.d, se muestran los perfiles de las líneas 1-8 seleccionadas en la fotografía (a). En la Figura 4.9. e, se muestran los perfiles de las líneas 1-4 seleccionadas en la fotografía (b). En la Figura 4.9.f, se muestran los perfiles de las líneas 1-6 seleccionadas en la fotografía (c).

Con el objeto de analizar la influencia de la energía en el proceso de ablación láser de fs sobre el blanco de Al, se decidio fabricar controladamente NPs de Al en agua, utilizando valores entre 50 y $500 \mu \mathrm{J}$. Para cada una de las muestras, se obtuvieron espectros de extinción y a partir de estos espectros de extinción se determinaron las especies y distribuciones de tamaño de cada una de las especies, utilizando la espectroscopia de extinción. Las especies y distribuciones de tamaño se resumen en la Tabla 4.2 , 

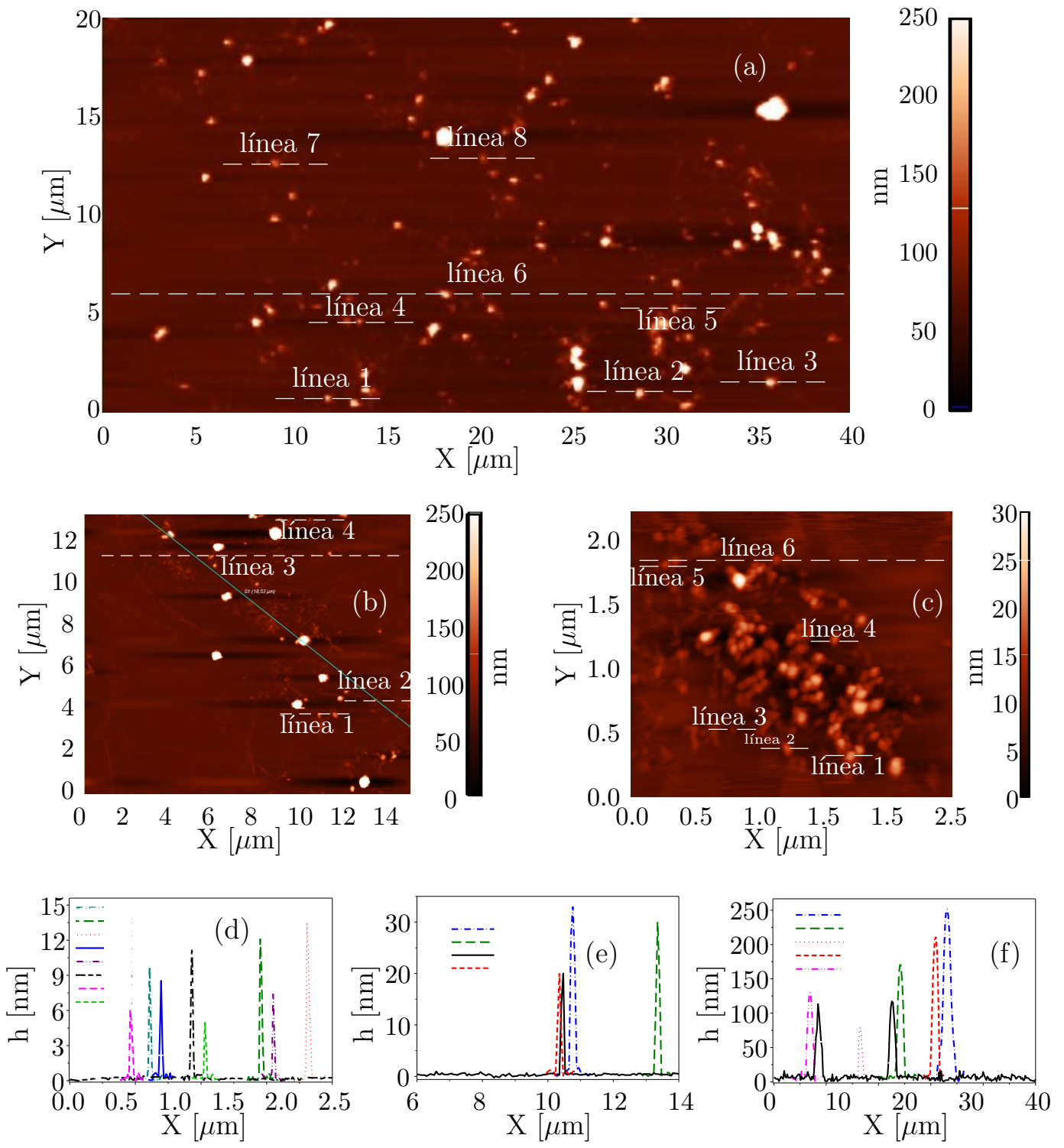

Figura 4.9: Fotos AFM de NPs de Al fabricadas por ablación láser en agua y perfiles de algunas líneas seleccionadas.

Se puede apreciar que las NPs que se encuentran compuestas solo de Al son más pequeñas que las que se obtienen de $\mathrm{Al}_{-}-\mathrm{Al}_{2} \mathrm{O}_{3}$ y, como se mostró en el Capítulo 2 , la oxidación es mayor en las NPs mas pequeñas. Por tanto es de esperar que las NPs mas grandes tengan una capa de óxido más pequeña, que en algunos casos puede ser la correspondiente capa de Langmuir (cerca de 0.3nm). En el caso de los espectros de extinción la diferencia entre las NPs de núcleo desnudo y las que tienen una capa tan delgada de óxido, es pequeña por tanto es posible que las NPs que se describen como 
de Al tengan esta pequeña capa de óxido.

\begin{tabular}{|c|c|c|c|c|c|c|c|c|c|c|c|}
\hline Especie & \multicolumn{2}{|c|}{ Al } & \multicolumn{2}{|c|}{ Al- $\mathrm{Al}_{2} \mathrm{O}_{3}$} & \multicolumn{3}{|c|}{ Aire-Al } & \multicolumn{3}{|c|}{ Aire- $\mathrm{Al}_{2} \mathrm{O}_{3}$} & \multirow[b]{2}{*}{$\%$} \\
\hline Energía & $\begin{array}{c}R_{m} \\
{[\mathrm{~nm}]}\end{array}$ & $\sigma$ & $\begin{array}{c}R_{m} \\
{[\mathrm{~nm}]}\end{array}$ & $\sigma$ & $\%$ & $\begin{array}{c}R_{m} \\
{[\mathrm{~nm}]}\end{array}$ & $\sigma$ & $\%$ & $\begin{array}{c}R_{m} \\
{[\mathrm{~nm}]}\end{array}$ & $\sigma$ & \\
\hline 50 & 29.4 & 0.78 & 10.4 & 0.42 & 19.8 & 16.2 & 0.66 & 20.4 & - & - & - \\
\hline 100 & 30.2 & 0.82 & 9.40 & 0.36 & 20.4 & 12.4 & 0.64 & 20.0 & 10.0 & 0.22 & 24.0 \\
\hline 150 & 29.2 & 0.78 & 10.2 & 0.44 & 18.8 & 14.4 & 0.66 & 19.4 & 9.86 & 0.20 & 22.4 \\
\hline 200 & 29.6 & 0.62 & 10.2 & 0.44 & 20.2 & 12.6 & 0.58 & 18.6 & 10.2 & 0.22 & 22.6 \\
\hline 250 & 30.0 & 0.82 & - & - & - & 16.4 & 0.62 & 19.0 & 10.0 & 0.18 & 20.4 \\
\hline 300 & 20.4 & 0.58 & - & - & - & 11.8 & 0.60 & 19.4 & 10.2 & 0.22 & 20.8 \\
\hline 350 & 19.8 & 0.24 & - & - & - & 13.8 & 0.96 & 20.4 & 10.4 & 0.20 & 23.6 \\
\hline 500 & 10.8 & 0.94 & 11.3 & 1.03 & 19.4 & 15.1 & 0.67 & 20.3 & - & - & - \\
\hline
\end{tabular}

Tabla 4.2: Resumen de la distribución de tamaños de NPs fabricadas por ablación láser sobre un blanco de $\mathrm{Al}$ en agua, para distintas energías de ablación

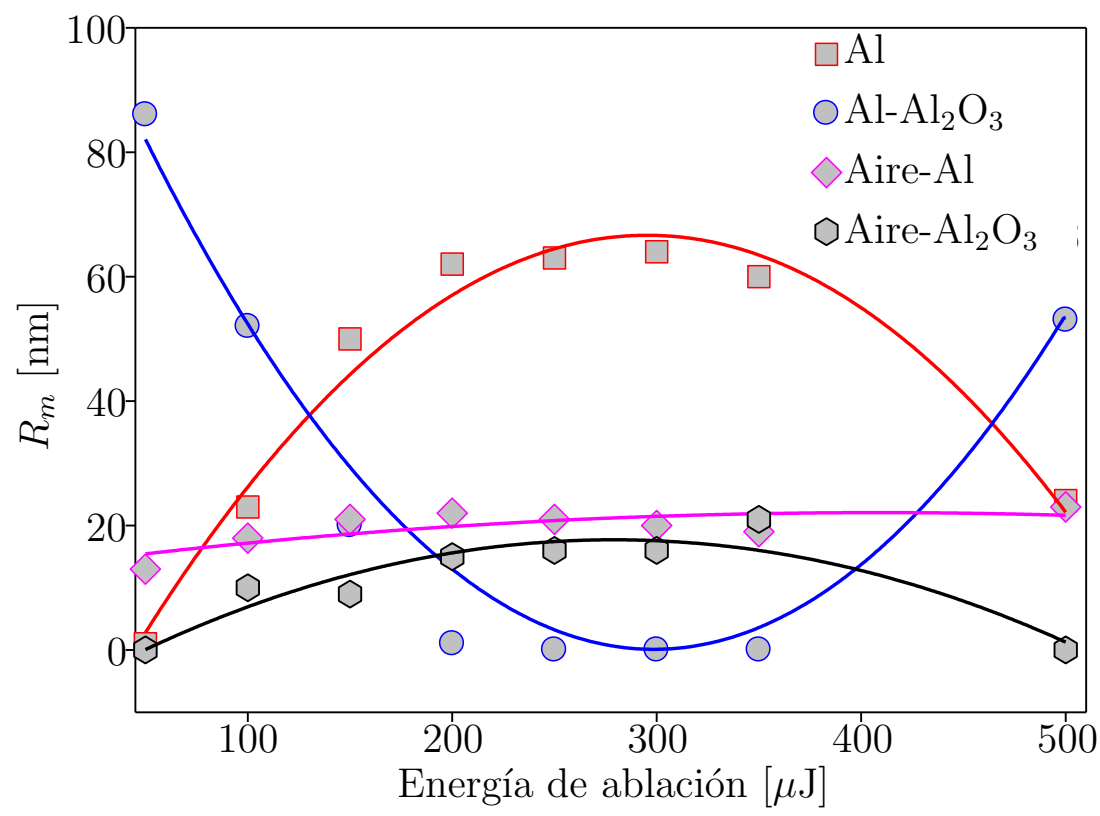

Figura 4.10: Radios de las diferentes especies de NPs obtenidas utilizando la ablación láser sobre un blanco de $\mathrm{Al}$ en agua, en función de la energía de ablación.

Los porcentajes de abundancia de las especies presentes se grafican en la Figura 4.10, en función de la energía de ablación. Se observa que al incrementar la energía de ablación, se incrementa el número de NPs de Al (éstas pueden presentar una pequeña capa de óxido), las cuales a su vez son de mayor tamaño, como se observa en la Figura. El mismo fenómeno ocurre con las NPs de la forma Aire- $\mathrm{Al}_{2} \mathrm{O}_{3}$. Lo antes descrito ocurre 
a expensas de la disminución de las NPs de $\mathrm{Al}$ con una capa de $\mathrm{Al}_{2} \mathrm{O}_{3}$ (capa de óxido superior a la capa de Langmuir). Las NPs de Aire-Al representan un porcentaje similar para todas las energías de ablación. Todo lo antes descrito ocurre hasta cierta energía umbral a partir de la cual se invierte el fenómeno de formación de especies de NPs antes descrito. La energía umbral a la que se invierte el fenómeno es cercana a los $300 \mu \mathrm{J}$.

\subsection{Caracterización de nanopartículas no esféricas}

En las secciones anteriores determinamos distribuciones de tamaños de NPs esféricas, utilizando la teoría de Mie para describir la extinción de las NPs esféricas de núcleo desnudo o con cubiertas. Cuando las NPs no son esféricas no se puede utilizar la teoría de Mie para describir la extinción de las NPs. En este caso se recurre a métodos numéricos. Uno de los más utilizados corresponde a la aproximación de dipolos discretos denominada DDA por sus siglas en inglés Discrete Dipole Aproximation.

\subsubsection{Aproximación de dipolos discretos}

La aproximación DDA consiste en considerar a una NE como formada por dipolos discretos, los cuales, al ser sometidos a un campo incidente (por lo general una onda plana), se polarizan (adquieren un momento dipolar). Una vez determinado el momento

dipolar de cada uno de los dipolos que constituyen la estructura (ver Apéndice C), se pueden calcular las eficiencias de absorción, extinción y scattering, así como los campos en cada una de las regiones $[\mathbf{1 0 0} \mathbf{1 0 4}$.

$$
\begin{gathered}
C_{e x t}=\frac{4 \pi k}{\left|\mathbf{E}_{0}\right|^{2}} \sum_{k=1}^{N} \operatorname{Im}\left\{\mathbf{E}_{i n c, k}^{*} \cdot \mathbf{p}_{k}\right\} \\
C_{a b s}=\frac{4 \pi k}{\left|\mathbf{E}_{0}\right|^{2}} \sum_{k=1}^{N}\left\{\operatorname{Im}\left[\mathbf{p}_{k} \cdot\left(\alpha^{-1}\right)^{*} \mathbf{p}_{k}^{*}\right]-\frac{2}{3} k^{3}\left|\mathbf{p}_{k}\right|^{2}\right\} \\
C_{s c a}=C_{e x t}-C_{a b s}
\end{gathered}
$$

Para analizar la validez de la aproximación DDA, comparamos los espectros de 
extinción de NPs esféricas de Au de $20 \mathrm{~nm}$ y Ag de $30 \mathrm{~nm}$, como se ve en la Figura 4.11.a. El espectro en rojo es obtenido utilizando la teoría de Mie y el espectro en azul es obtenido utilizando la aproximación DDA. Se observa que las características más relevantes, como la ubicación y el ancho del pico plasmónico, son equivalentes utilizando la teoría de Mie o la aproximación DDA. Similares conclusiones se observan en la Figura 4.11.b donde se comparan la teoría de Mie y la aproximación DDA para NPs esféricas con núcleos de $\mathrm{Al}$ y Au de $20 \mathrm{~nm}$ de radio con una cubierta de $10 \mathrm{~nm}$ de espesor de $\mathrm{Al}_{2} \mathrm{O}_{3}$.
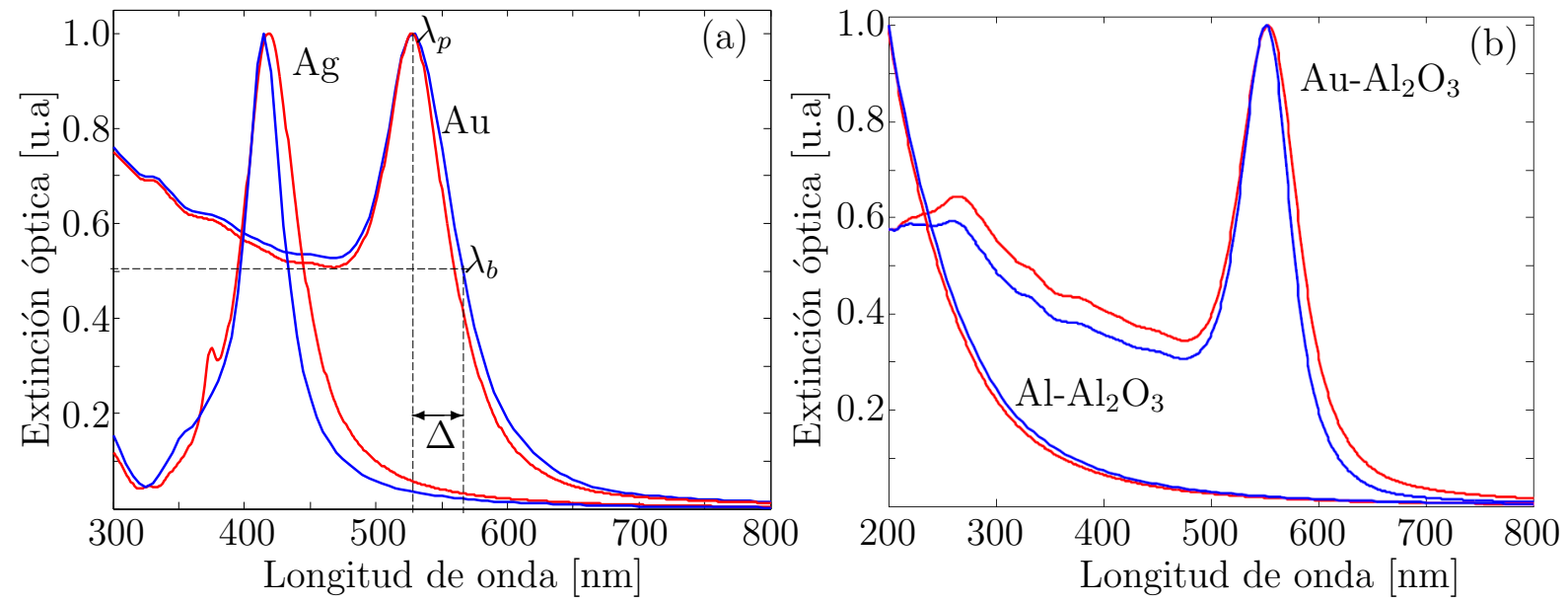

Figura 4.11: (a)Espectro de extinción de NPs de Au de $20 \mathrm{~nm}$ de radio y de Ag de 30 $\mathrm{nm}$ de radio, en rojo utilizando la teoría de Mie y en azul utilizando la DDA. (b)Espectro de extinción de NPs de Au y Al de $20 \mathrm{~nm}$ de radio con una cubierta de $10 \mathrm{~nm}$ de $\mathrm{Al}_{2} \mathrm{O}_{3}$, en rojo utilizando la teoría de Mie y en azul utilizando la DDA.

En la Figura 4.11, hemos validado el método DDA para un radio especifico. Si variamos el valor del radio en NPs de Au, y comparamos la ubicación y el ancho del pico plasmónico (Figura 4.12.a), observamos que en el rango de 10 a $50 \mathrm{~nm}$ de radio de las NPs, los valores obtenidos utilizando la aproximación DDA son muy cercanos a los obtenidos utilizando la teoría de Mie. En la Figura 4.11.b se muestra la distribución espacial de campo eléctrico para una NP esférica de Au de $15 \mathrm{~nm}$ de radio, considerando una longitud de onda de incidencia de $520 \mathrm{~nm}$. 


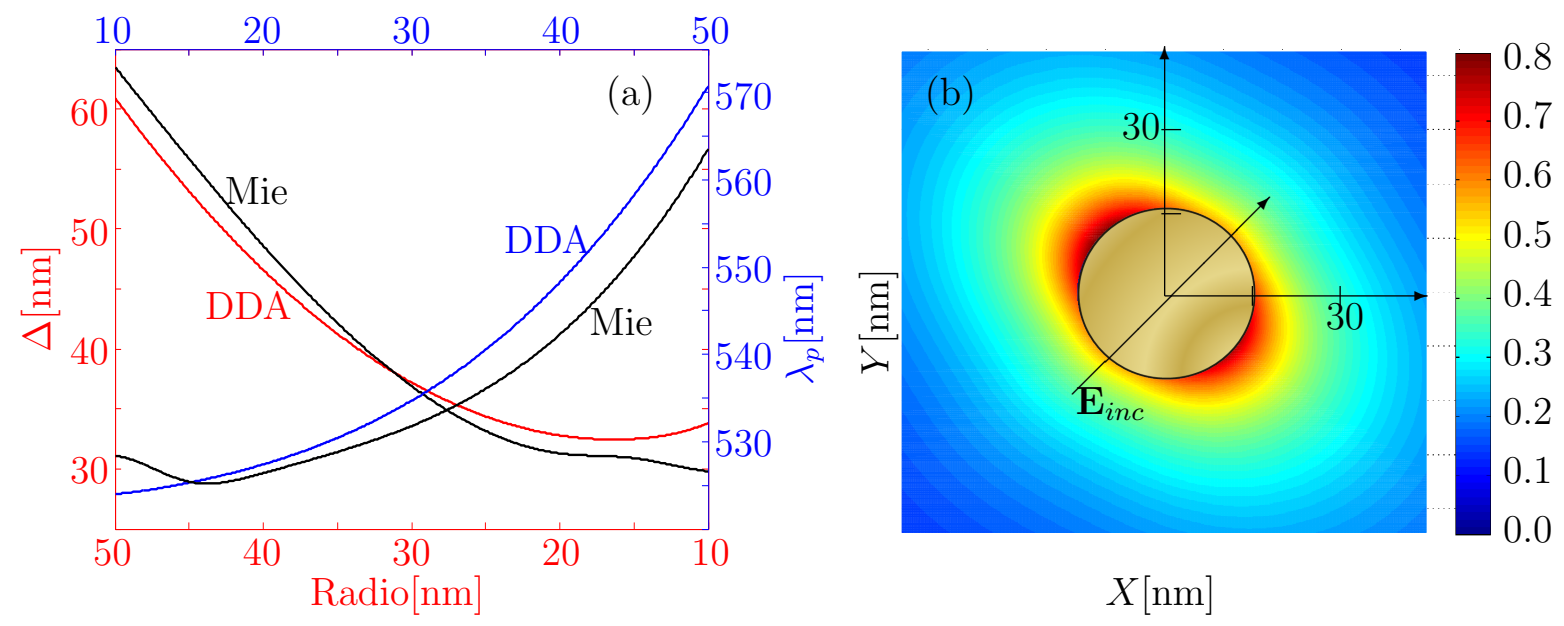

Figura 4.12: (a) En la escala roja se observa la evolución del ancho del pico plasmónico para NPs de $\mathrm{Au}$, en negro utilizando la teoría de Mie y en rojo utilizando la DDA; en la escala azul, se gráfica la ubicación del pico plasmónico en negro utilizando la teoría de Mie y en azul la DDA (b) Campo dispersado por una NP esférica de Au de $15 \mathrm{~nm}$ de radio en un campo aplicado en la dirección $[1,1,0]$ a $520 \mathrm{~nm}$ de longitud de onda.

Una vez validado el método de DDA utilizando la teoría de Mie, estamos en condiciones de extenderlo a otras geometrías. Como primer ejemplo, lo aplicaremos a NPs cúbicas de Au de lado $L=20,40$ y $60 \mathrm{~nm}$, considerando un campo aplicado en la dirección $[1,1,0]$ y con vector de propagación en la dirección $[0,0,1]$.

En este caso, se obtienen los espectros ilustrados en la Figura 4.13,a, donde, al igual que para las NPs esféricas, a medida que se incrementa el tamaño de la NP (incrementa el lado $L$ ) el pico plasmónico se desplaza a longitudes de onda más largas. También se observa que el ancho medio de la resonancia plasmónica en el caso de NPs cúbicas es menor que el equivalente en NPs esféricas de diámetro igual a $L$. La Figura 4.13.b sintetiza la evolución de la ubicación y ancho del pico plasmónico para NPs esféricas de radio entre 10 y 50 nm y para NPs cúbicas de lado entre 20 y 100 nm.

El campo dispersado en una NP cubica de lado $L=10 \mathrm{~nm}$, se muestra en la Figura 4.14.b, donde se observa claramente el efecto del vértice. En la Figura 4.14.a, se muestra la ubicación del pico plasmónico en NPs cubicas de $20 \mathrm{~nm}$ de lado, cubierta de $\mathrm{SiO}_{2}$ y espesor variable $0,5\left(L_{2}-L_{1}\right)$. Este resultado es comparado con el equivalente a NPs esféricas de $10 \mathrm{~nm}$ de radio y espesor variable $R_{2}-R_{1}$. 


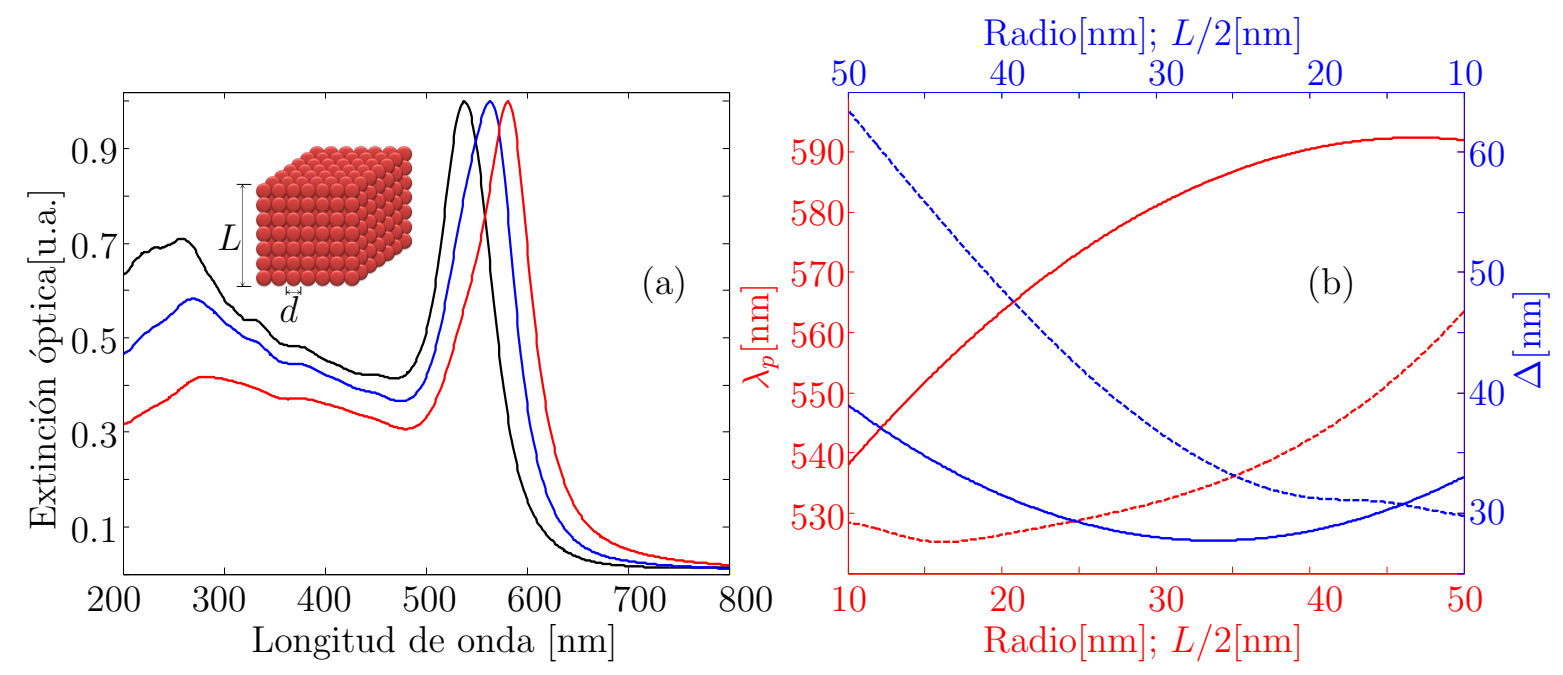

Figura 4.13: (a) Espectro de extinción de NPs cúbicas de Au de 20,40 y 60 nm de longitud utilizando la DDA. (b) Evolución de la ubicación y ancho del pico plasmónico, en línea continua para NPs cúbicas y en linea segmentada para NPs esféricas en rojo la ubicación del pico plasmónico y en azul el ancho del pico plasmónico.
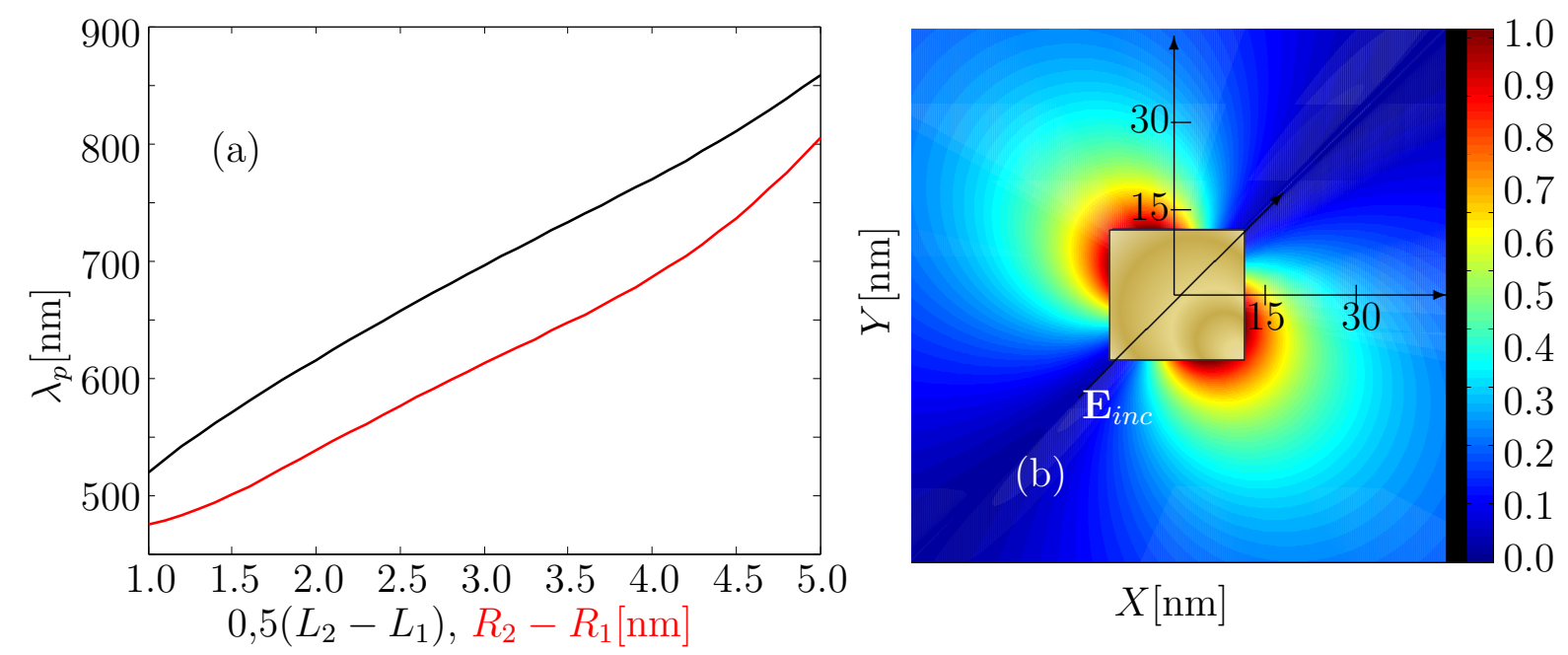

Figura 4.14: (a) Ubicación del pico plasmónico para NPs de $\mathrm{Au}$ con núcleo de $\mathrm{SiO}_{2}$, en negro para NPs cubicas y en rojo para NPs esféricas. (b) Campo en una $\mathrm{NP}$ cúbica de $10 \mathrm{~nm}$ de lado, con campo aplicado en la dirección $[1,1,0]$ y $\lambda=520 \mathrm{~nm}$.

Para NPs de forma cuboidal (nanoantenas cuboidales), se tienen soluciones aproximadas del campo en el interior de la nanoantena, considerando que la polarización en el interior del cuboide es constante [33]. Como esta aproximación no es correcta los 
autores realizan correcciones considerando cargas inducidas en los vértices del cuboide, pero esta solución sigue siendo una muy débil aproximación a la solución del problema.

Como se ha observado hasta el momento una de las virtudes de la DDA se encuentra el hecho de que puede ser utilizada para diversas geometrías, como se muestra en la Figura 4.15 donde se grafican los espectros de extinción de NPs de Na con diversas formas (cubo, cubo octaedro, octaedro truncado, icosaedro, decaedro y esfera). Cada una de las NPs tiene un volumen tal que el radio efectivo es $2.5 \mathrm{~nm}$. La dirección del campo eléctrico incidente sobre la nanoestructura es [100].

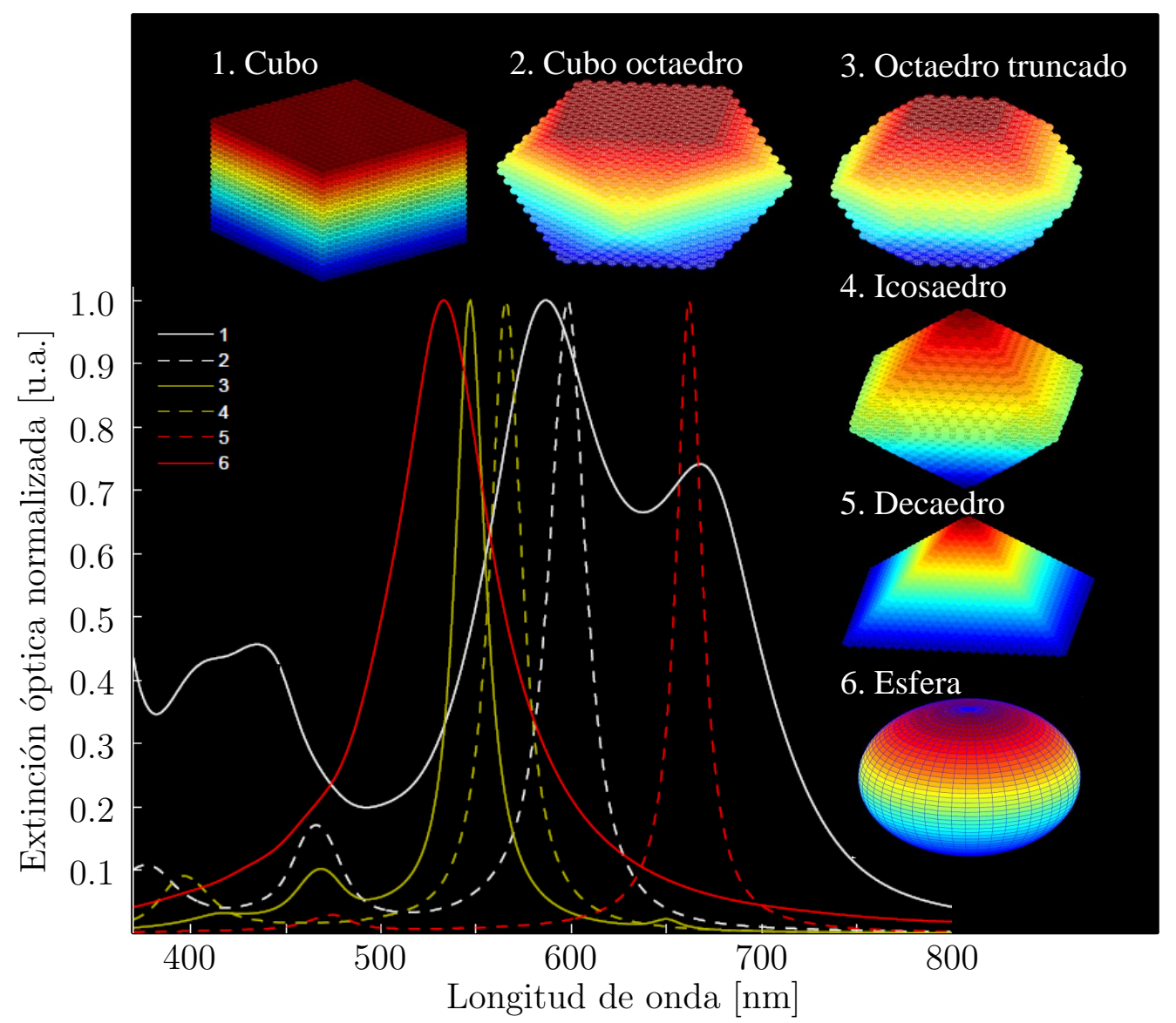

Figura 4.15: Espectro de extinción de NPs de sodio de diferente geometría y sus respectivas discretizaciones para un radio efectivo de $2.5 \mathrm{~nm}$.

Variando la dirección de la onda incidente sobre la estructura nanométrica y dependiendo de la geometría de la misma se pueden excitar diferentes resonancias 
plasmónicas como se muestra en la Figura 4.16 donde se varia la dirección del campo eléctrico incidente y se observan dos modos predominantes uno cerca de $400 \mathrm{~nm}$ que corresponde al modo en la dirección $z$ y otro cercano a $670 \mathrm{~nm}$ que corresponde al modo en la dirección $y$; en las direcciones intermedias conviven ambos modos de resonancia.

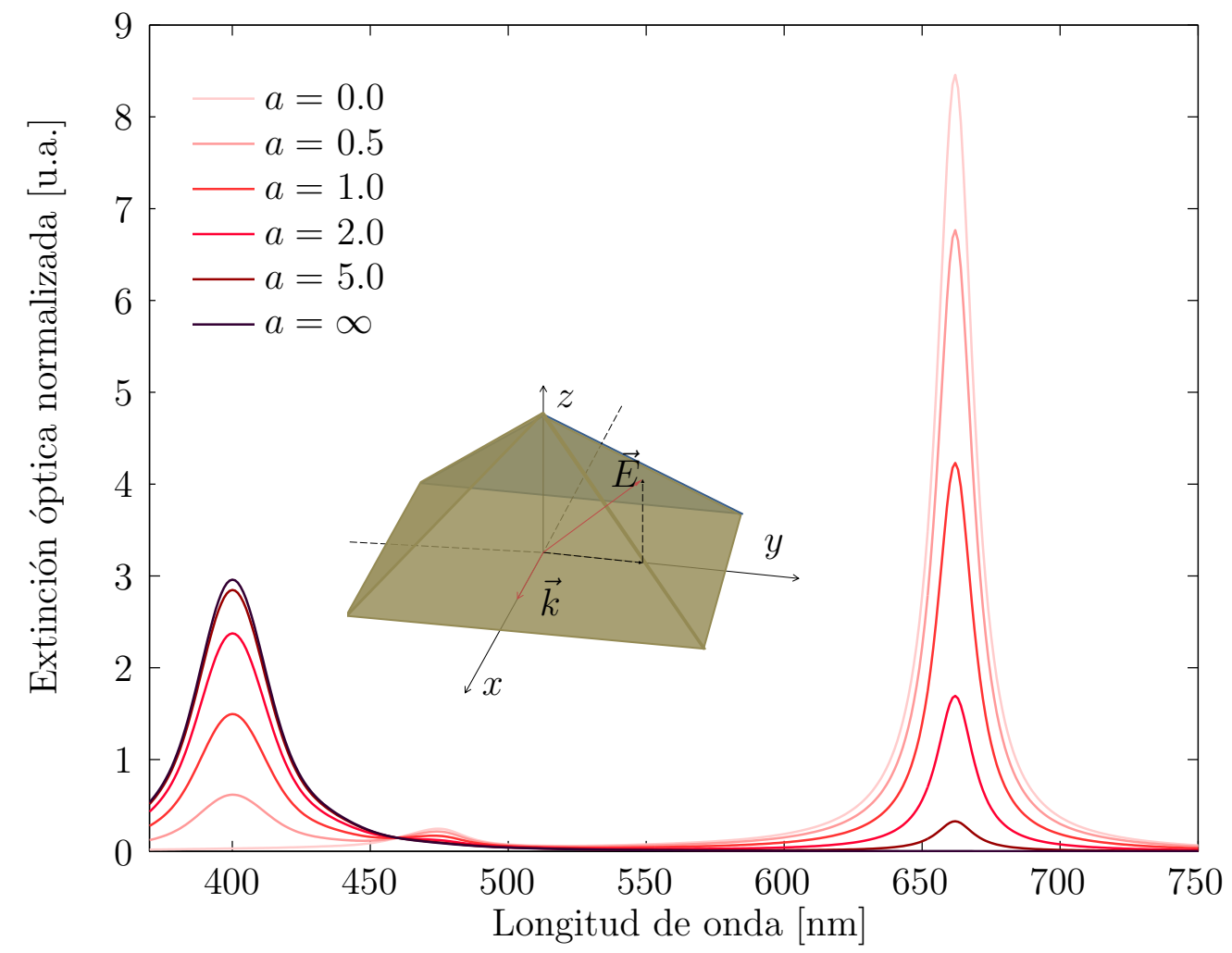

Figura 4.16: Espectro de extinción de NPs en forma de decaedro de Na donde el campo eléctrico incide en la dirección $[01 a]$ para diferentes valores de $a=0,0.5$, $1.0,2.0,5.0$ y $\infty$.

Cuando $a=0$ el campo eléctrico incide en la dirección $y$, mientras que en el caso $a=\infty$ el campo eléctrico incide en la dirección $z$ y en los demás casos el campo eléctrico incide en el plano $y z$. Al incrementar el valor del parámetro $a$, se favorece la resonancia ubicada cerca de $400 \mathrm{~nm}$, debido al incremento de la componente del campo en la dirección $z$ y por ende se desfavorece la resonancia ubicada cerca de $670 \mathrm{~nm}$. 


\subsubsection{Caracterización de nanopartículas no esféricas de $\mathrm{Na}$}

Al igual que en el caso de NPs esféricas, para las NPs de forma arbitraria, realizamos un gráfico de la ubicación y ancho del pico plasmónico de las NPs en función de los parámetros de la distribución log-normal.
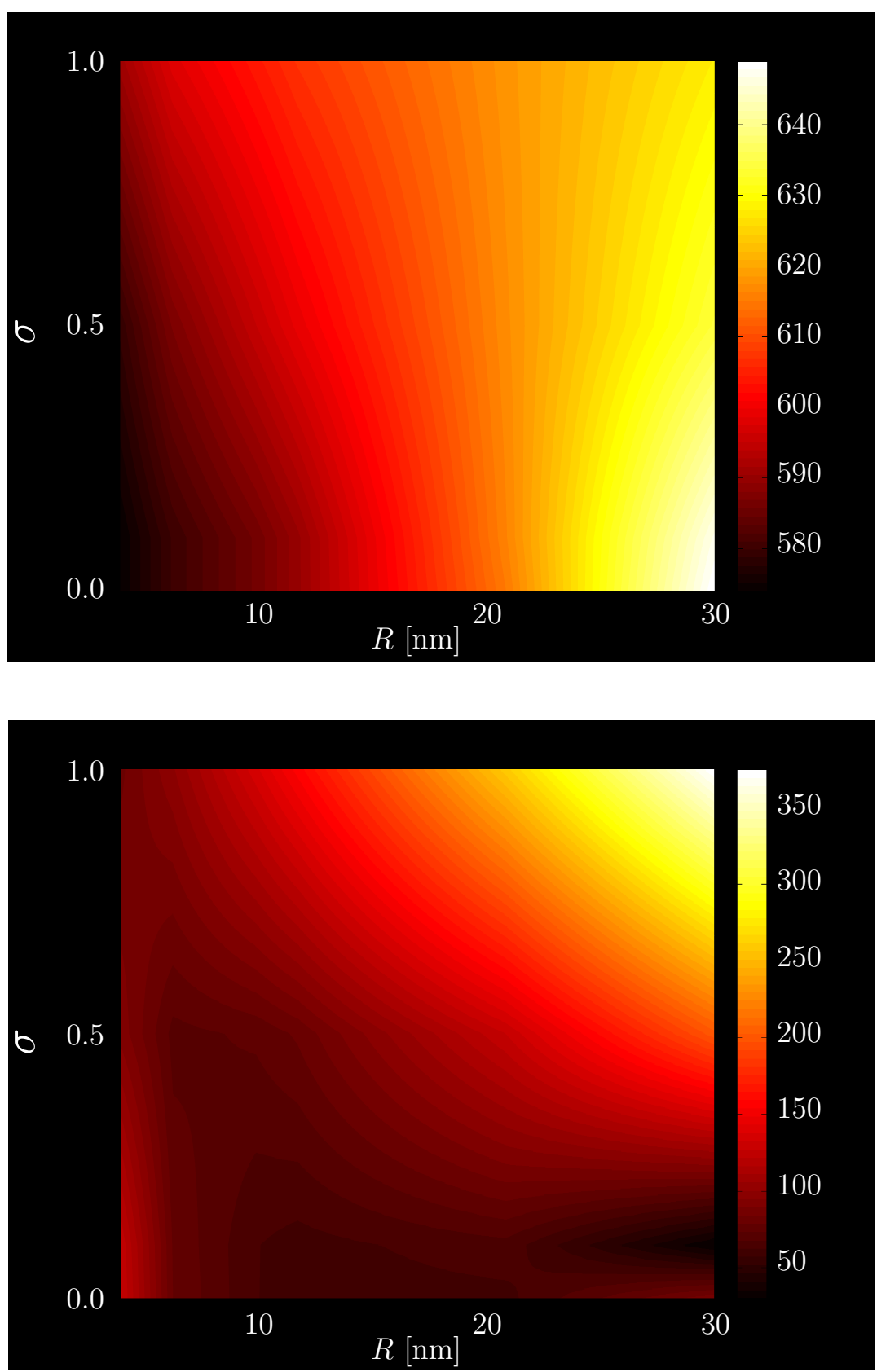

Figura 4.17: Ubicación y ancho del pico plasmónico de Nps de Na en forma de octaedro truncado. 
En la Fígura 4.17 se muestra la variación de la ubicación y el ancho del pico plasmónico de NPs de Na en forma de octaedro truncado para diferentes distribuciones de tamaño $\left(R_{m}\right.$ y $\left.\sigma\right)$. Estos graficos se utilizan para determinar las distribuciones de tamaño de NPs de Na obtenidas por irradiación de láminas finas de monocristales de haluros alcalinos, obtenidas de muestras naturales de cloruro de sodio a partir de la aglomeración de defectos producidos por daño radiativo en las muestras, donde la forma de octaedro truncado resulto ser la más adecuada para describir las NPs.

En la Figura 4.18 se grafican las curvas de nivel para la ubicación $(608.8 \mathrm{~nm})$ y el ancho $(81.7 \mathrm{~nm})$ del pico plasmónico correspondientes al espectro experimental en azul de la Figura 4.19. Además, se muestran dos curvas adicionales correspondientes a \pm $0.8 \mathrm{~nm}$ en en el caso de la ubicación del pico plasmónico y $\pm 0.7 \mathrm{~nm}$ para el caso del ancho dl pico plasmónico. El cruce de estas curvas representa la solución al problema (los valores de $\sigma$ y $R_{m}$ ), solución que se muestra en el circulo amplificado.

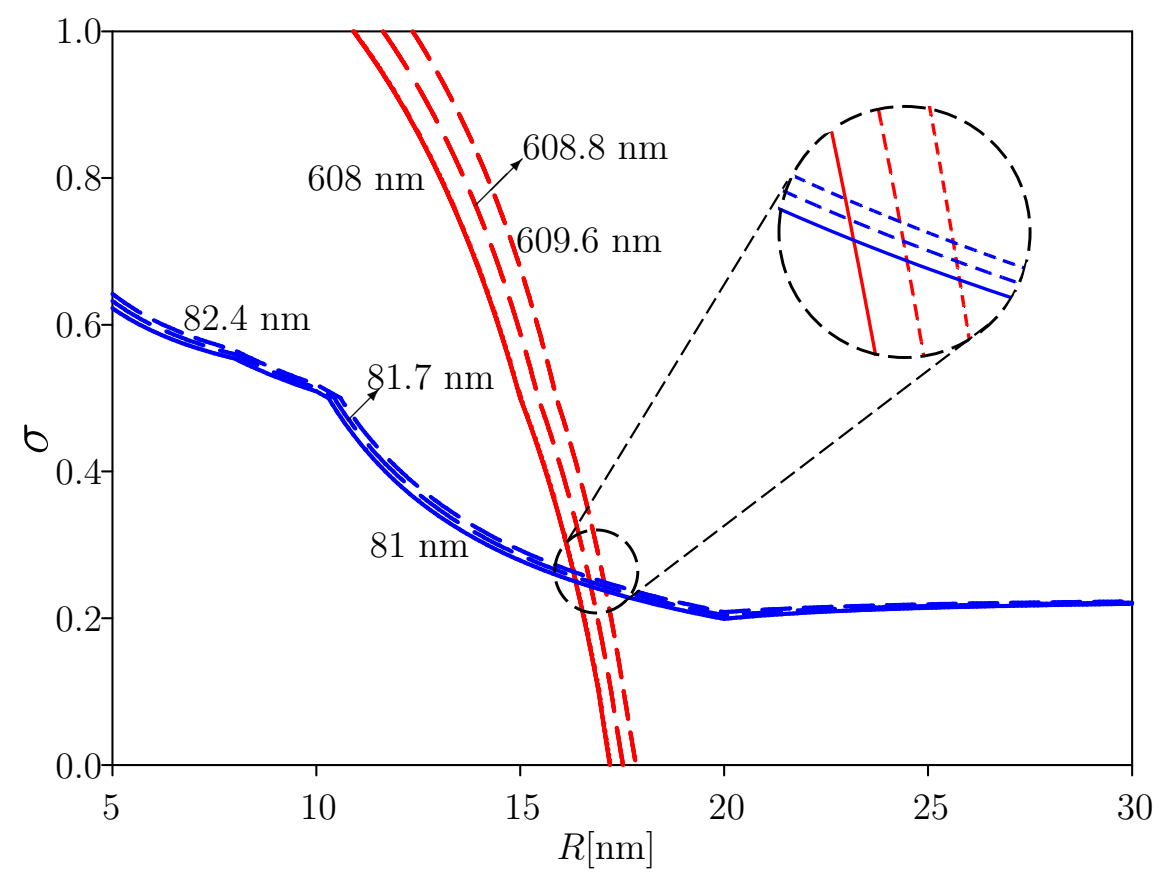

Figura 4.18: Curvas de nivel para Nps de $\mathrm{Na}$ con la ubicación y el ancho del pico plasmónico del espectro experimental. 
El espectro ajustado que presenta menor error con respecto al experimental se muestra en la Figura 4.19 en rojo. En este el radio efectivo más probable es $R m=16,8 \mathrm{~nm}$ y su distribución de tamaños se encuentra caracterizada por $\sigma=0,25$.

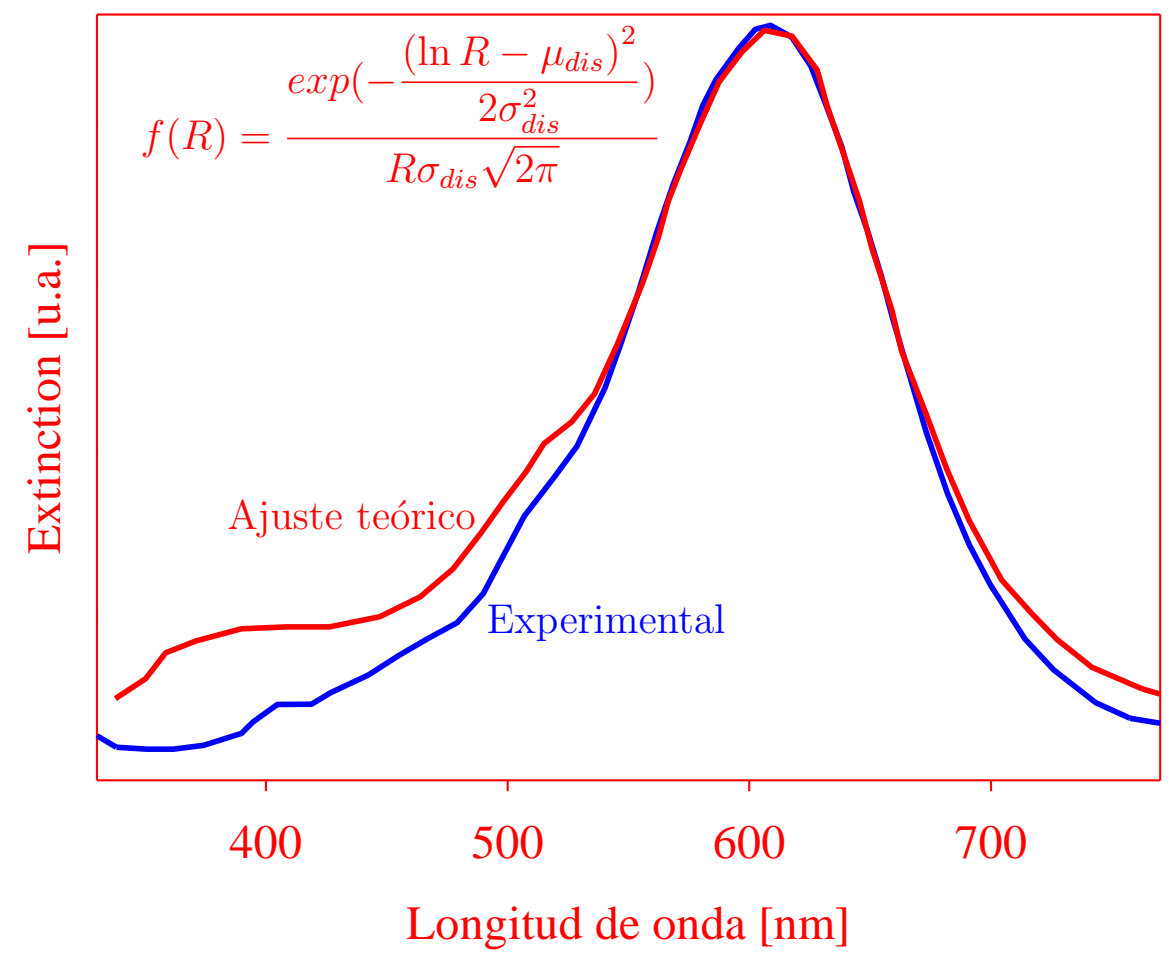

Figura 4.19: Espectros experimental y ajuste teórico de Nps de Na. En rojo el espectro teórico y en azul el espectro experimental.

Cabe aclarar que, tanto las muestras como los espectros experimentales de las mismas fueron obtenidos en una etapa previa a esta tesis por el Dr Jorge Tocho y su becario doctoral Alvaro Carrera.

Para resumir, este Capítulo 4 está dedicado a mostrar la caracterización de tamaño, estructura y configuración de los coloides antes generados a través del ajuste de los espectros experimentales de extinción óptica con la teoría desarrollada en el Capítulo 2. Para el 
caso de NPs no esféricas, se implementó el código de aproximación por dipolos discretos (DDA) que permite determinar la distribución espacial de campo en forma numérica. El mismo fue validado para el caso de NPs esféricas, comparándolo con los cálculos analíticos provenientes de teoría de Mie. Como aplicación, se muestra la determinación de la distribución de tamaño de NPs de sodio en una matriz de haluros alcalinos a través del ajuste del espectro de extinción. 


\section{Capítulo 5 Refuerzo de campo en nanopartículas esféricas}

Avanzando estos tres pasos, llegarás más cerca de los Daimones:

Primero: Habla con verdad.

Segundo: No te dejes dominar por la cólera.

Tercero: Da, aunque no tengas más que muy poco que dar.

Buda.

En los capítulos anteriores hemos estudiado las características plasmónicas de NPs de núcleo desnudo y núcleo con cubierta en función del tamaño, y de qué manera estas propiedades pueden ser utilizadas convenientemente para determinar la distribución de tamaño por extinción óptica en muestras específicas. El conocimiento de estas propiedades plasmónicas resulta importante para su utilización en aplicaciones de diversas áreas de la ciencia y la tecnología $[\mathbf{1 0 5}-\mathbf{1 1 4}$. En los últimos años, ha habido un gran interés en la síntesis y caracterización del NPs con núcleo y doble cubierta de la forma metal-dieléctrico-metal debido a sus diversas aplicaciones en catálisis [115, 116], SERS biocompatible [117], liberación de fármacos [118], diagnóstico y terapia de tumores [119], detección de contaminantes [120,121], mejora del refuerzo de campo cercano SERS [122] y amplificación óptica paramétrica a nanoescala [123].

Estas NPs núcleo-doble cubierta se han sintetizado con éxito utilizando métodos químicos y asistidos por láser [117, 124, 125]. Algunas de las aplicaciones antes mencionadas como así también otras relacionadas con microscopía [126], nanoantenas [127, 128] y amplificación de ganancia en celdas solares [129], se basan en el 
incremento de campo en las cercanías de la nanoestructura. Este refuerzo es causado por las oscilaciones de los electrones que se encuentran en la banda de conducción (plasmones) cuando una onda electromagnética incide sobre la nanoestructura metálica. Normalmente se utilizan estructuras de formas no esféricas (triángulos, pirámides, etc.) a fin de lograr incrementos de campo importantes. Mostraremos en este capítulo que es posible obtener grandes refuerzos de campo utilizando NPs esféricas del tipo núcleo-doble cubierta, que en la práctica son más sencillas de obtener.

Para estudiar este tipo de refuerzos, se suele utilizar la llamada "aproximación dipolar" por ser un método simplificado de cálculo analítico. Sin embargo, debe tenerse precaución al aplicarlo, ya que puede conducir en algunos casos a resultados erróneos como se verá en la sección siguiente.

En este capítulo se desarrolla la teoría de Mie exacta para NPs esféricas núcleo-doble cubierta, partiendo de las soluciones de la ecuación vectorial de ondas en coordenadas

esféricas. A continuación se construyen expresiones para los campos en cada una de las regiones de la NP, como una combinación de las soluciones de la ecuación vectorial de ondas. Considerando las condiciones de contorno apropiadas en cada interfaz se determinan los coeficientes de los campos en cada una de las regiones. Finalmente con las expresiones de los campos en cada una de las regiones se determina la absorción y la extinción de las NPs. Este desarrollo específico se muestra en el Apéndice B para no distraer la continuidad del presente capítulo.

Cabe mencionar que en el caso de estructuras más complejas no esféricas, la solución analítica puede no existir y es necesario reemplazarla por estrategias basadas en cálculos numéricos, como por ejemplo la aproximación de dipolos discretos (DDA) 100 104 vista en el Capítulo 4 .

\subsection{Teoría de Mie vs aproximación dipolar y efecto de la corrección por tamaño}

Trabajos recientes referidos a estudios teóricos sobre NPs de núcleo metálico y doble recubrimiento dieléctrico-metal, se abocan al análisis de la respuesta plasmónica y la 
dependencia del refuerzo de campo con el tamaño relativo núcleo-recubrimiento para el caso específico del sistema $\mathrm{Au}-\mathrm{SiO}_{2}-\mathrm{Au}$, motivados principalmente por aplicaciones biocompatibles de este tipo de NPs $130-132$.

$\mathrm{Hu}$ et al. [130 analizaron la respuesta espectral y las características de dispersión angular de la mencionada estructura $\mathrm{Au}-\mathrm{SiO}_{2}-\mathrm{Au}$ el código de Mie para mostrar las similitudes y diferencias entre las propiedades ópticas de la estructura estudiada y la clásica $\mathrm{SiO}_{2}-\mathrm{Au}$, aunque sin tener en cuenta las correcciones de tamaño intrínsecas de la función dieléctrica del metal. Por otra parte, Wu et al. [131] investigaron la influencia del tamaño de núcleo y la capa dieléctrica intermetálica en las propiedades de resonancia del plasmón de $\mathrm{NPs}$ de $\mathrm{Au}-\mathrm{SiO}_{2}-\mathrm{Au}$, utilizando también Mie, pero con una función dieléctrica del Au corregida por tamaño solo para electrones libres. Finalmente, Zhu et al. [132 estudiaron el efecto del núcleo de Au en la mejora de campo local de nanocubiertas $\mathrm{Au}-\mathrm{SiO}_{2}$-Au usando la aproximación electrostática (dipolar), aunque los diámetros utilizados están en el orden de varias decenas de nanómetros, tamaños para los cuales esta aproximación pierde su validez.

Como dijimos anteriormente, se debe tener especial cuidado tanto en la consideración de las correcciones por tamaño como en la utilización de las diferentes aproximaciones (dipolar o cuasiestática). Esto último se relaciona con las condiciones aplicadas al llamado "parámento de tamaño $x$ " $(x=2 \pi R N / \lambda)$, donde $N$ es el índice de refracción del medio circundante y $\lambda$ es la longitud de onda incidente en vacío. Mientras la condición $x<<1$ considera sólo contribuciones dipolares al campo dispersado por la NP (aproximación dipolar), la condición $m x<<1$ (siendo $m$ el índice de refracción relativo complejo del metal [13]), tiene en cuenta contribuciones multipolares de orden superior (aproximación cuasiestática). En ambas aproximaciones, el campo dentro de la partícula se considera uniforme. A pesar de que las palabras cuasi-estática y dipolar se usan con frecuencia de manera indistinta, la última no puede describir con precisión características plasmónicas cuadrupolares o superiores para tamaños de unas pocas decenas de nanómetros. En estos casos, es conveniente utilizar la teoría Mie. 
Un resultado experimental típico sobre NPs de oro obtenido en nuestro laboratorio muestra que cuando se incrementa el tamaño de las NP (por ejemplo de $10 \mathrm{~nm}$ a 80 $\mathrm{nm}$ ), se obtienen espectros con una resonancia plasmónica fuertemente desplazada hacia el rojo. Sin embargo, la expresión de aproximación dipolar sólo es capaz de reproducir correctamente los espectros correspondientes a pequeños tamaños (del orden de $20 \mathrm{~nm})$. El plasmón obtenido utilizando esta aproximación no presenta desplazamientos al rojo importantes para radios mayores. Este ejemplo de NPs de oro puede inducir a la engañosa idea de que todas las NPs metálicas de menos de $20 \mathrm{~nm}$ de tamaño pueden tratarse con seguridad en la aproximación dipolar. En este sentido es necesario señalar que la correcta ubicación de la longitud de onda de la resonancia plasmónica (así como resonancias de orden superior) depende no sólo del tamaño de la NP sino también del metal del que está fabricada.
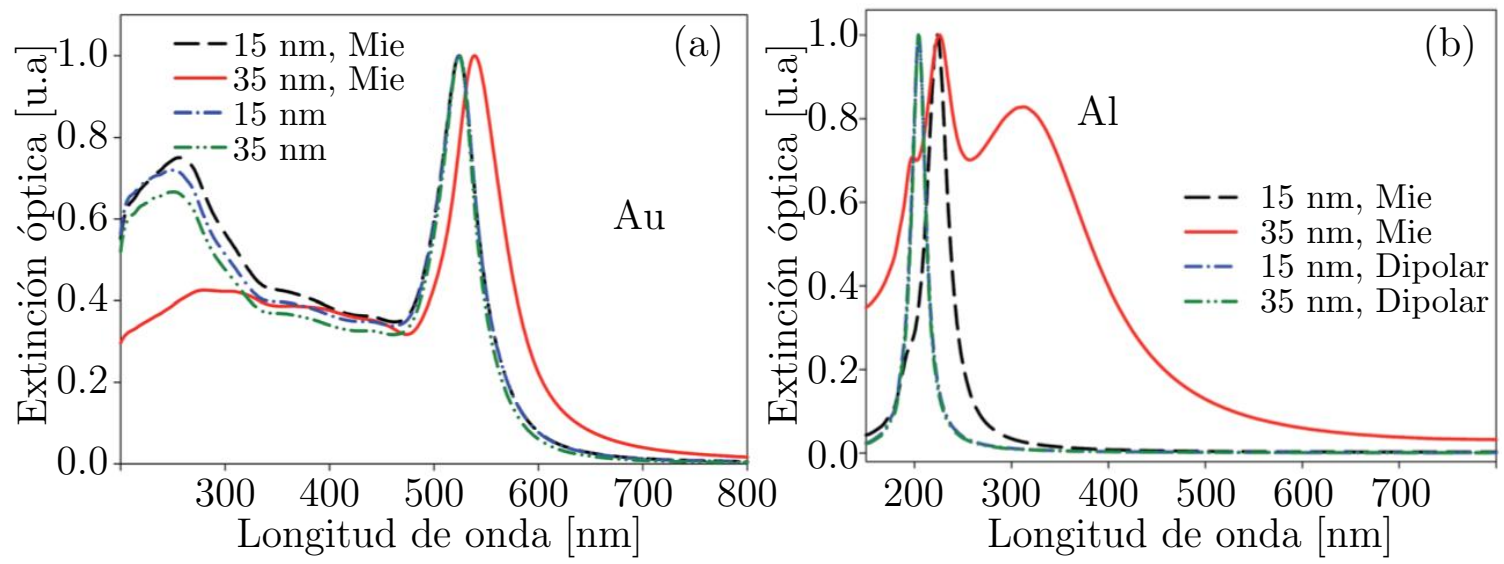

Figura 5.1: Comparación entre espectros de extinción de NPs esféricas de núcleo desnudo, calculado usando la teoría de Mie y su aproximación dipolar para (a) Oro y (b) aluminio, para dos tamaños.

Para fines comparativos, la Figura 5.1 ilustra este punto para el caso de espectros de extinción de NPs de núcleo desnudo de Au (a) y Al (b) para dos tamaños diferentes. Para el caso del Au, se encuentra el resultado intuitivamente esperado: para pequeños tamaños del radio (15 nm), la aproximación dipolar (líneas azul de segmentos y punto) y la teoría Mie (líneas de segmentos negra) producen espectros similares, con resonancias plasmónicas coincidentes, mientras que para tamaños más grandes (35 nm) amboa 
cálculos producen resultados bien diferentes: la aproximación dipolar continúa dando la resonancia plasmónica en la misma longitud de onda que para $15 \mathrm{~nm}$, mientras que un cálculo con teoría de Mie (línea continua roja) produce el resultado correcto de plasmón corrido hacia longitudes de onda mayores, debido al incremento del tamaño. Para el caso de NPs de Al se observa una diferencia aún mas notable entre los espectros de extinción obtenidos con aproximación dipolar y teoría de Mie. Para el radio de 15 nm, la aproximación dipolar produce un único pico plasmónico en $200 \mathrm{~nm}$ mientras que un cálculo con teoría de Mie lo determina en 220nm. Al aumentar el tamaño a 35 nm, las diferencias son aún más notables, ya que la aproximación dipolar produce un espectro de extinción prácticamente superpuesto al correspondiente a $15 \mathrm{~nm}$ mientras que la teoría de Mie completa muestra la aparición de resonancias de orden superior, además del típico corrimiento al rojo del plasmón original, ubicado ahora en $300 \mathrm{~nm}$.

Cuando se consideran los espectros de extinción de NPs con doble cubierta, se observan también notables diferencias dependiendo del tipo de metal que forma el núcleo. En la Figura 5.2, se ilustran los espectros de extinción de NPs con doble cubierta, para el caso de núcleo de oro (panel a) y núcleo de aluminio (panel b), ambos con una capa intermedia de sílice y una cubierta externa de oro. Los radios de núcleo utilizado en los cálculos para ambos metales son de $R_{1}=2 \mathrm{~nm}$ y $R_{1}=22 \mathrm{~nm}$, siendo la relación entre los tres radios $R_{1}, R_{2}$ y $R_{3}$, dada por $R_{2}=1.5 R_{1}$ y $R_{3}=2 R_{1}$. Para el caso del núcleo de oro, tanto la aproximación dipolar (curva azul) como teoría de Mie (curva roja) predicen dos resonancias plasmónicas, observando que para $R_{1}=2$ $\mathrm{nm}$ los espectros se superponen (línea continua). Para $R_{1}=22 \mathrm{~nm}$, la aproximación dipolar predice los picos plasmónicos en las mismas longitudes de onda que para el caso de $R_{1}=2 \mathrm{~nm}$, mientras que el cálculo de Mie predice el esperado corrimiento al rojo de ambas resonancias por aumento de tamaño, como así también un aumento en el FWHM del pico de menor longitud de onda. 

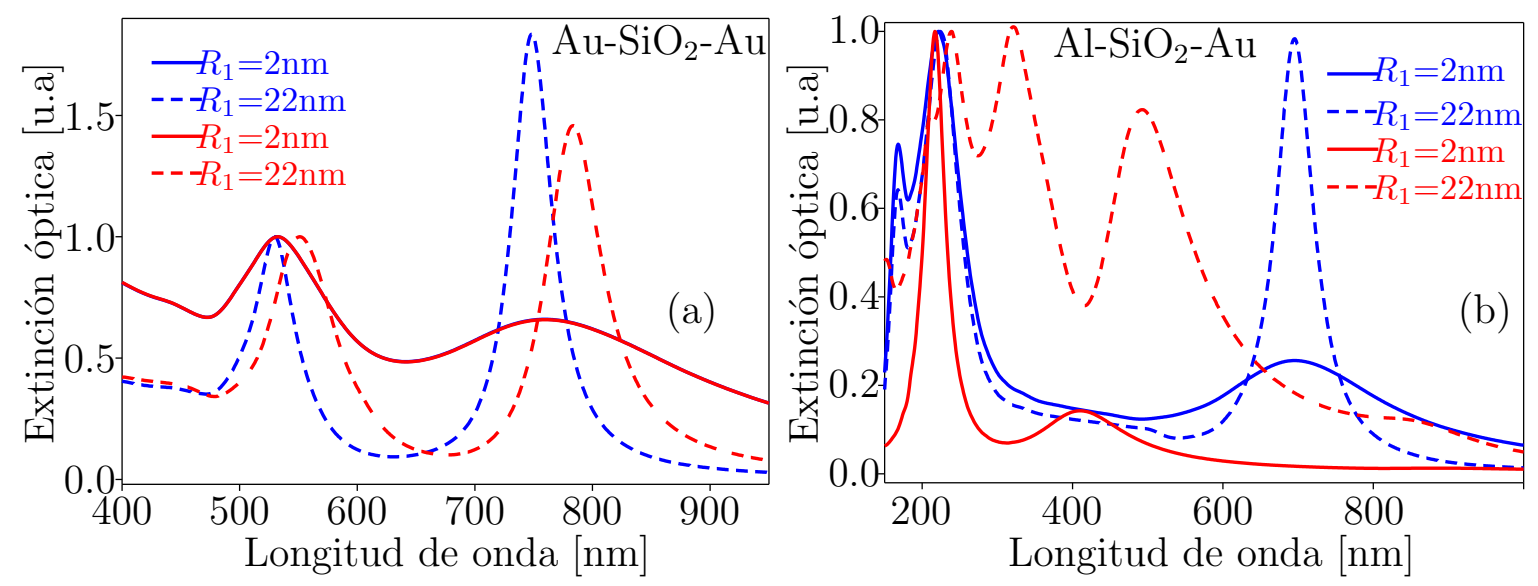

Figura 5.2: Comparación entre la teoría de Mie y la aproximación dipolar para NPs con núcleo y doble cubierta. (a) $\mathrm{Au}-\mathrm{SiO}_{2}-\mathrm{Au}$ y (b) $\mathrm{Al}_{-} \mathrm{SiO}_{2}-\mathrm{Au}$. En ambos casos la relación de radios es: $R_{2}=1,5 R_{1}$ y $R_{3}=2 R_{1}$. Se muestran en rojo los espectros de extinción utilizando teoría de Mie y en azul utilizando la aproximación dipolar. En línea continua para $R_{1}=2 \mathrm{~nm}$ y en línea de segmentos para $R_{1}=22 \mathrm{~nm}$.

Si consideramos ahora un núcleo de aluminio en lugar de oro, se observan marcadas diferencias respecto del caso anterior, como se muestra en el panel (b). Cuando se utiliza la aproximación dipolar para calcular los espectros de extinción, con los mismos tamaños que en el panel (a), se predicen dos resonancias plasmónicas, que se encuentran aproximadamente en $200 \mathrm{~nm}$ y en $700 \mathrm{~nm}$. Sin embargo, cuando se utiliza teoría de Mie, para $R_{1}=2 \mathrm{~nm}$, el pico plasmónico de mayor longitud de onda se encuentra desplazado hacia el azul (ubicado en $400 \mathrm{~nm}$ ), mientras que el otro permanece casi $\sin$ modificaciones. Para $R_{1}=22 \mathrm{~nm}$, la teoría de Mie predice un corrimiento hacia el rojo de todas las resonancias, incluyendo las de tipo multipolar (pequeño hombro aproximadamente en $850 \mathrm{~nm}$ ), mientras que la aproximación dipolar predice erróneamente una única resonancia plasmónica cerca de $700 \mathrm{~nm}$.

Las conclusiones anteriores sugieren que la condición $m x<<1$ ocurre para tamaños mucho menores en el caso del Al, comparado con el Au. Esto significa que se debe ser cuidadoso a la hora de utilizar la aproximación dipolar basado sólo en el hecho de que la NP sea menor que determinado tamaño (típicamente $R<10 \mathrm{~nm}$, sino que se debe tener en cuenta el metal específico del que está conformada. Teniendo en cuenta estos resultados, de ahora en mas utilizaremos teoría de Mie para los próximos cálculos de campos y refuerzos. 
Otro aspecto fundamental en las predicciones de las resonancias plasmónicas lo constituye la adecuada descripción de la función dieléctrica como función de la longitud de onda. Como mostramos en el Capítulo 2, cuando disminuye el radio de la NP se hace necesario corregir la función dieléctrica por tamaño para tomar en cuenta la modificación del camino libre medio de los electrones de conducción y la modificación de la distribución de estados electrónicos en las bandas de energía para electrones ligados. La corrección de la función dieléctrica por tamaño muestra mayor influencia en los espectros de extinción de NPs de menores tamaños. Esto puede verse en la Figura 5.3 donde se grafican espectros de extinción para NPs con núcleo de oro y dos cubiertas: una de $\mathrm{SiO}_{2}$ y la otra de $\mathrm{Au}$,con radios diferentes pero manteniendo la cubierta intermetálica con espesor constante de $5 \mathrm{~nm}$. Para mejor comparación, los espectros se encuentran normalizados a la resonancia plasmónica de menor longitud de onda.

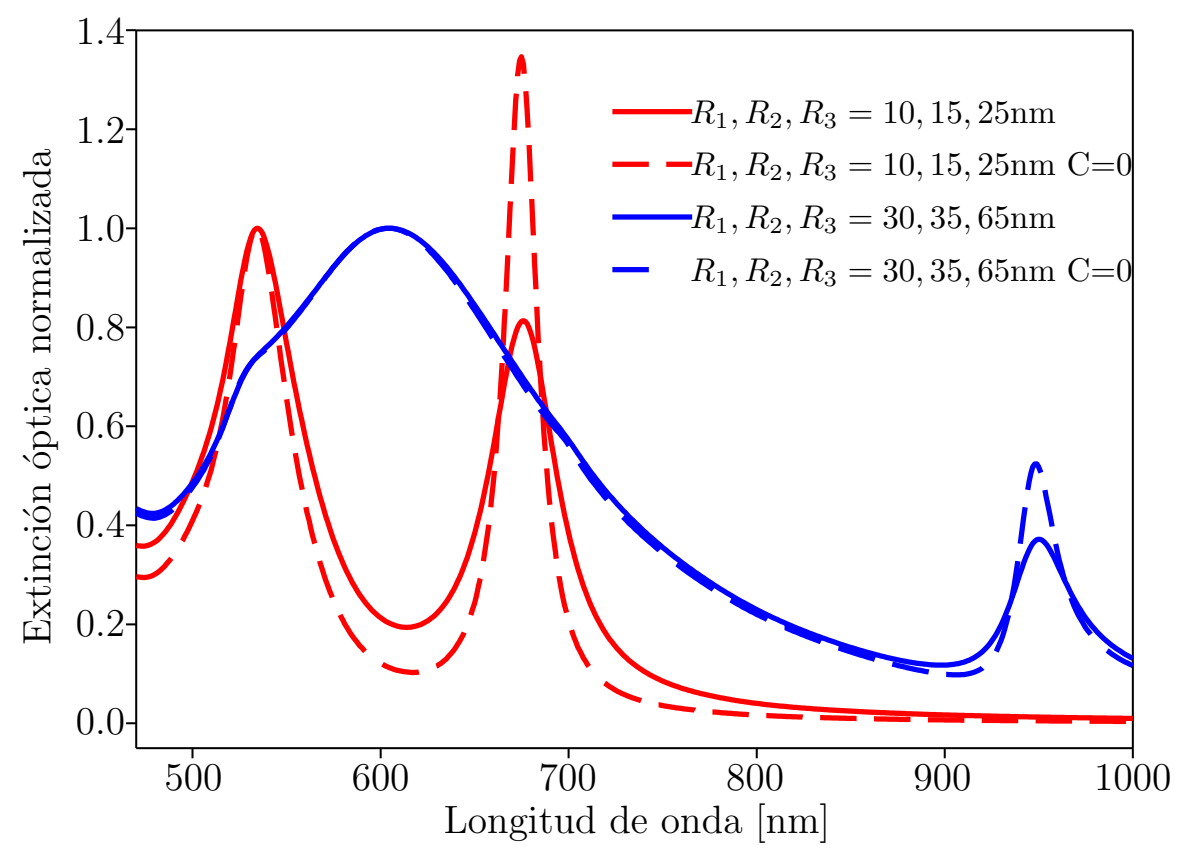

Figura 5.3: Influencia de la corrección de la función dieléctrica en los espectros de extinción de NPs $\mathrm{Au}-\mathrm{SiO}_{2}-\mathrm{Au}$, utilizando la función dieléctrica corregida por tamaño en línea continua y sin corregir (línea segmentada) para $R_{1}=10$ $\mathrm{nm}, R_{2}=15 \mathrm{~nm}$ y $R_{3}=25 \mathrm{~nm}$, en rojo y para $R_{1}=30 \mathrm{~nm}, R_{2}=35 \mathrm{~nm} \mathrm{y}$ $R_{3}=65 \mathrm{~nm}$ en azul. 
Para $R_{1}=10 \mathrm{~nm}$, cuando la función dieléctrica no se corrige por tamaño (línea roja segmentada), se observa una extinción mayor para la longitud de onda mas larga. Lo anterior implica que se debe corregir la función dieléctrica por tamaño [130, 133, 134]. $\mathrm{Al}$ aumentar el tamaño al valor $R_{1}=30 \mathrm{~nm}$, se obtienen los espectros de extinción indicados en color azul, en línea continua para la función dieléctrica corregida y en línea segmentada para la función dieléctrica sin corrección. En este caso, los respectivos espectros de extinción son más cercanos que en su contraparte $R_{1}=10 \mathrm{~nm}$, lo que indica que la corrección de la función dieléctrica es menos importante cuanto mas grande es el tamaño de las NPs.

\subsubsection{Influencia del metal del núcleo}

En la Figura 5.4 se muestran espectros de extinción de NPs de la forma metal- $\mathrm{SiO}_{2}-\mathrm{Au}$, donde el metal del núcleo puede ser $\mathrm{Au}, \mathrm{Ag}, \mathrm{Cu}$ ó Al. En los cálculos, la capa externa se mantiene de Au debido al creciente interés en aplicaciones biológicas, para lo que se requiere biocompatibilidad de la capa más externa.

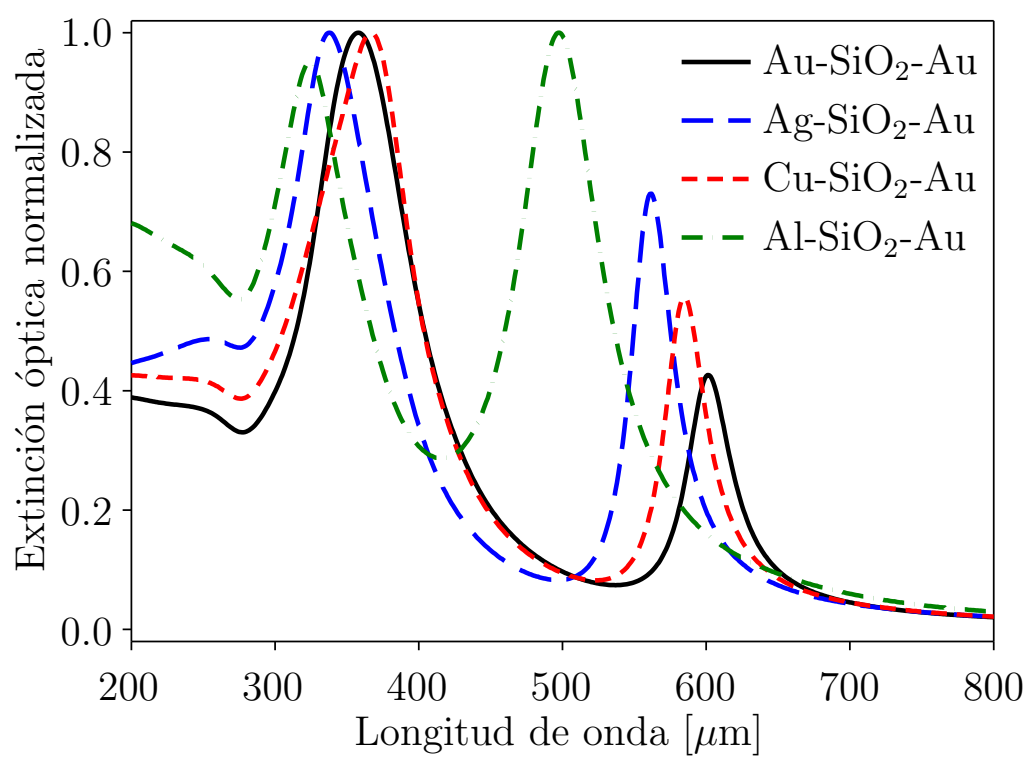

Figura 5.4: Espectros de extinción de $\mathrm{NPs}$ de metal- $\mathrm{SiO}_{2}-\mathrm{Au}$, para núcleos de $\mathrm{Au}, \mathrm{Ag}$, $\mathrm{Cu}$ y Al. 
Manteniendo las mismas relaciones de dimensiones que la utilizada en la (Figura $5.3\left(R_{1}, 1.5 R_{1}, 2 R_{1}\right)$, se observa que, para el mismo tamaño, en todos los casos el pico de longitud de onda menor se encuentra en el ultravioleta y el de mayor longitud de onda en el visible. Lo espectros de NPs con núcleo de Al poseen resonancias más cercanas entre sí y, a diferencia de los otros metales sus amplitudes son similares.

\subsubsection{Modelo de Hibridación}

Las resonancias de NPs tipo núcleo y doble cubierta mostradas en la Figura 5.4 pueden interpretarse como una combinación de resonancias individuales de las estructuras más simples que constituyen a la NP compuesta. Este enfoque sigue la línea del llamado modelo de hibridación utilizado en Química, basado en la interacción de orbitales atómicos dentro de una molécula para formar nuevos orbitales híbridos. La hibridación no es un fenómeno físico en sí mismo, sino simplemente una descripción matemática que combina los orbitales atómicos componentes de tal manera que los nuevos orbitales moleculares (híbridos) poseen propiedades generales que son razonablemente consistentes con lo observado para un amplio rango de moléculas.

Una descripción análoga fue desarrollada por Prodan et al. [135 para el caso de NPs con núcleo dieléctrico y cubierta de oro con el objetivo de describir las propiedades plasmónicas de este tipo de nanoestructura compuesta, considerando la resonancia plasmónica de una esfera maciza de oro y una cavidad esférica de $\mathrm{SiO}_{2}$ en un medio circundante de oro. En esta sección se describe cómo obtener las resonancias plasmónicas de NPs de estructura núcleo-doble cubierta (metal-dieléctrico-metal) extendiendo el modelo de hibridación para este nuevo tipo de estructura. En el Apéndice D, se explica detalladamente este método, ilustrando como ejemplo funciones de resonancia para una NP esférica de núcleo dieléctrico y cobertura metálica. Generalizando este procedimiento, se puede obtener la condición de resonancia $F(\omega)=0$ para una NP de la forma núcleo-doble cubierta del tipo metal-dieléctrico-metal. 
La Figura 5.5 condensa los resultados que se desarrollan en el Apéndice D. En la parte superior de la Figura $\mathbf{5 . 5}$ se grafica la condición de resonancia para una NP esférica de Au de $10 \mathrm{~nm}$ de radio (trazo azul). El cruce de esta función con el eje $x$ corresponde a la longitud de onda de resonancia de esta NP desnuda $\left(\lambda_{s}\right.$ en panel central). En trazo rojo se muestra la condición de resonancia para una NP con núcleo dieléctrico y cubierta de $\mathrm{Au}$ de radios interior y exterior de $15 \mathrm{~nm}$ y $25 \mathrm{~nm}$ respectivamente. Se observan dos cortes con el eje $x$ que corresponden a las longitudes de onda de resonancia de la nanoestructura compuesta $\left(\lambda_{+}\right.$y $\lambda_{-}$en panel central).

El corte con el eje $x$ de menor longitud de onda no se observa porque los valores experimentales de la función dieléctrica del Au con el que contamos no están definidos en esa región. Finalmente en negro se ilustra la condición de resonancia para una NP de la forma núcleo-doble cubierta $\left(\mathrm{Au}-\mathrm{SiO}_{2}-\mathrm{Au}\right)$ de radios $10 \mathrm{~nm}, 15 \mathrm{~nm}$ y $25 \mathrm{~nm}$ respectivamente, está condición de resonancia presenta tres cortes con el eje $x$ que corresponden a las longitudes de onda de resonancia de la NP núcleo-doble cubierta.

La hibridación para el caso cuasiestático con $l=1$ (aproximación dipolar) entre el plasmón de la NP esférica de $\mathrm{Au}\left(\lambda_{s}=537.0 \mathrm{~nm}\right)$ y los picos plasmónicos de la nano-cubierta $\lambda_{ \pm}=187.8,572.2 \mathrm{~nm}$, ocasiona las frecuencias de resonancia denotadas por $\lambda_{ \pm}^{ \pm}$, como se muestra en el panel central de la Figura $\mathbf{5 . 5}$ y cuyo espectro de extinción se muestra en el panel inferior de la misma (Espectros de extinción). En línea gris delgada para la aproximación dipolar y en línea negra gruesa para la teoría de Mie. Los valores de cada una de las longitudes de onda utilizando la teoría de Mie son: $\lambda_{s}=$ $539.0 \mathrm{~nm}$ y $\lambda_{ \pm}=195.4,578.0 \mathrm{~nm}$.

Las longitudes de onda $\lambda_{ \pm}=187.8,572.2 \mathrm{~nm}$, son el resultado de la hibridación entre el plasmón de la esféra de $25 \mathrm{~nm}$ y la cavidad esférica de $15 \mathrm{~nm}$. Para estimar el valor $\lambda_{+}$se utilizó la función dieléctrica de H.J.Hagemann-1974 para Au medida de $3.542 \mathrm{~nm}$ hasta $281.8 \mathrm{~nm}$. 


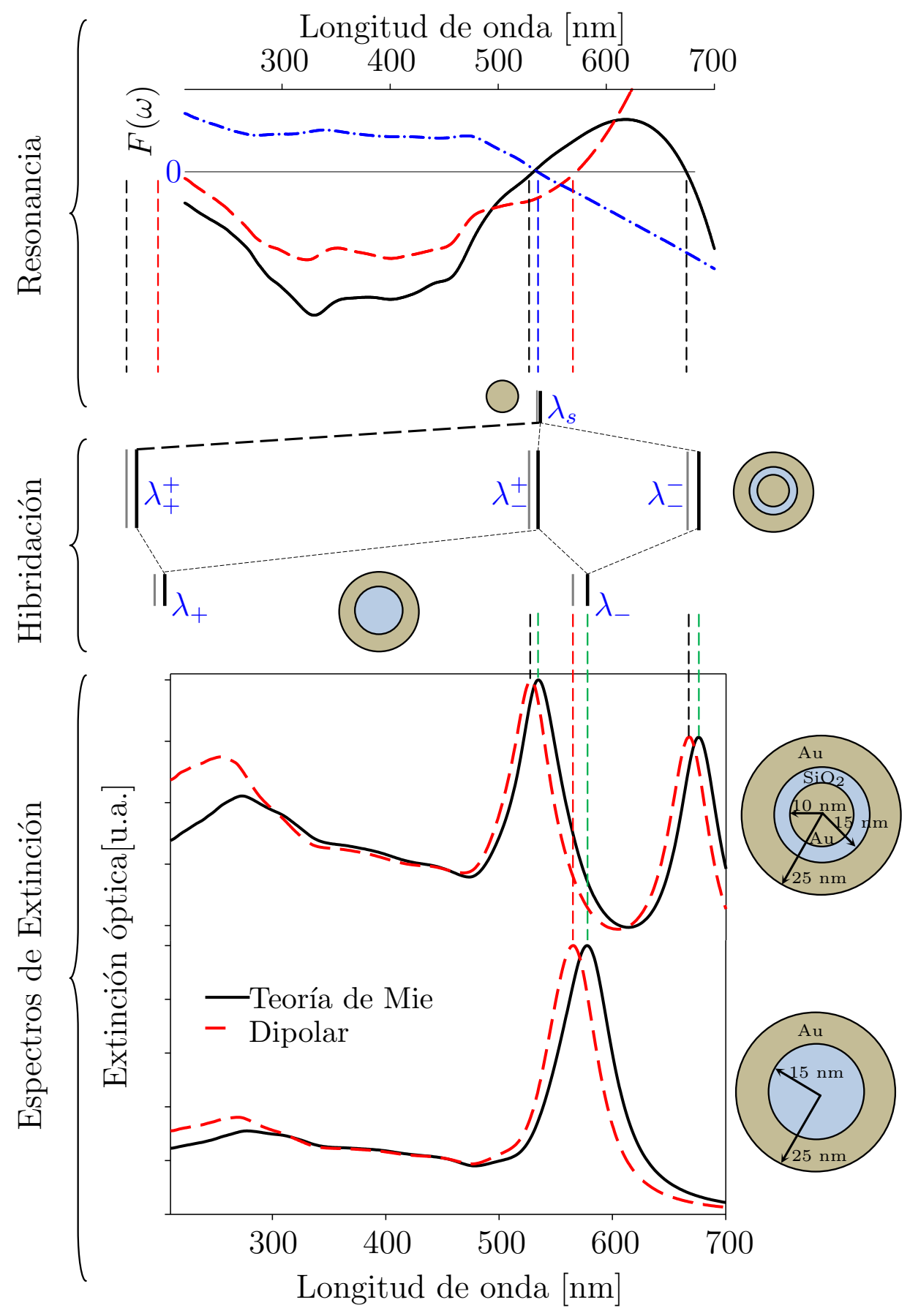

Figura 5.5: Condiciones de resonancia $(F(\omega)=0$ (parte superior), hibridación (parte central), y espectros de extinción (parte inferior) de nanopartículas esféricas de $\mathrm{Au}-\mathrm{SiO}_{2}-\mathrm{Au}$, para radios de 10,15 y $25 \mathrm{~nm}$ respectivamente. 


\subsection{Refuerzo de campo en estructuras esféricas con doble cubierta}

En la sección anterior, se estudió cómo cambia la extinción de NPs con la configuración metal- $\mathrm{SiO}_{2}-\mathrm{Au}$, observando la presencia de dos longitudes de onda de resonancia, que resultan de la hibridación del plasmón de la esfera central y las dos resonancias plasmónicas de la nanocubierta [135-137]. Recordando que las aplicaciones de estas NPs se basan en el refuerzo de campo en las cercanías de la misma [138], es importante conocer el refuerzo de campo en cada una de las regiones de los dieléctricos (intermetálico y exterior).

La distribución espacial de campo dentro y fuera de la Np se obtine de la teoría de Mie desarrollada para Nps con dos cubiertas. De esta distribución de campos se obtiene el valor máximo del campo, que al ser dividido por la amplitud del campo aplicado $\left(E / E_{0}\right)$ define el llamado el refuerzo de campo en la NPs.

Las distribuciones espaciales de campo para Nps en la configuración metal- $\mathrm{SiO}_{2}-\mathrm{Au}$ de 10, 15 y 25 nm de radio se muestran en las Figuras $\mathbf{5 . 6}$ a $\mathbf{5 . 9}$, considerando como metal del núcleo $\mathrm{Au}, \mathrm{Ag}, \mathrm{Cu}$ y $\mathrm{Al}$ respectivamente. La columna de la izquierda de estas figuras muestra el refuerzo de campo en el dieléctrico intermetálico mientras que la de la derecha corresponde al refuerzo en el dieléctrico externo. Esta separación se debe a la gran diferencia de valores de refuerzo, que no permitiría observar el campo en el exterior si se graficaran en forma conjunta. Se observa que el valor del refuerzo en la zona intermetálica es mucho mayor que en la zona exterior.

Para cada metal constituyente del núcleo se grafican los refuerzos de campo para tres longitudes de onda específicos: resonancia de menor longitud de onda (primera fila), resonancia de mayor longitud de onda (tercera fila) y en la zona del valle entre ambas resonancias (segunda fila). En estos gráficos se observa que para la longitud de onda más larga, la distribución de campo es mucho mas simétrica que la correspondiente a la longitud de onda más corta. Esta observación es causada por el hecho de que al

incrementar la longitud de onda estamos más cerca de la condición $m x \ll 1$ y por lo tanto habrá menos contribuciones de orden superior presentes en la distribución espacial 
del campo.

Los valores del índice de refracción relativo condicionan también la longitud de onda para la cual se cumple la condición $m x \ll 1$ : si un metal presenta un índice de refracción mayor que otro, se necesitará una longitud de onda mayor para alcanzar la condición antes descripta. Los índices de refracción de Au, Ag, $\mathrm{Cu}$ y Al a 600 nm son $0.249+3.074 i ; 0.055+4.010 i ; 0.494+2.962 i$ y $1.141+6.925 i$ respectivamente. En nuestro caso el mayor índice corresponde al Al y el menor a la Ag. Por esta razón, en el caso de la Ag la simetría se presenta a longitudes de onda en el rango visible, mientras que para el caso del Al deberá ocurrir a longitudes de onda en la zona del infrarrojo. 

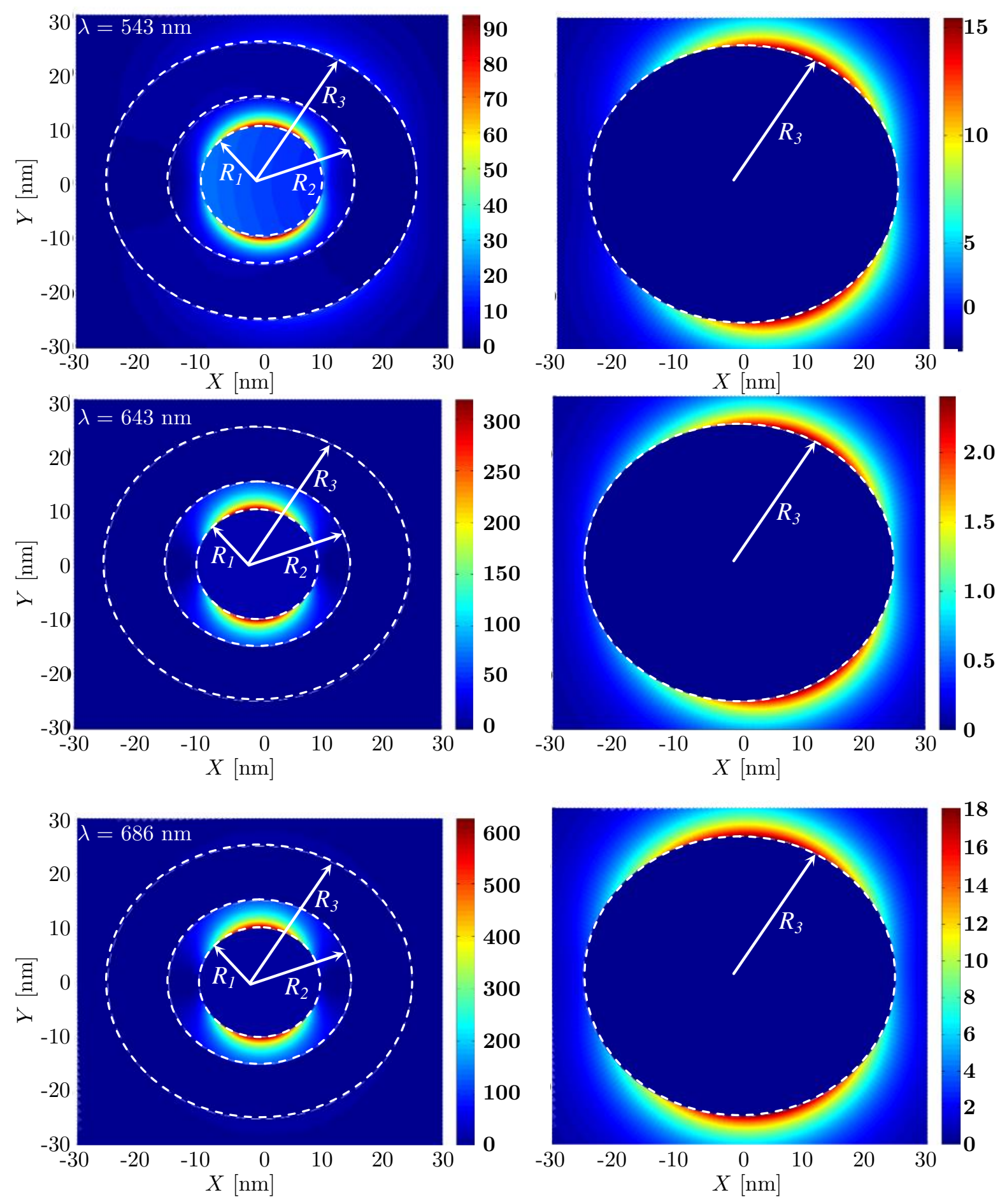

Figura 5.6: Refuerzo de campo $E / E_{0}$, para diferentes longitudes de onda $(543,643$ y $686 \mathrm{~nm}$ ) en una NP cuyo núcleo $R_{1}=10 \mathrm{~nm}$ es de Au, la primera capa es de $\mathrm{SiO}_{2}$ de radio $R_{2}=15 \mathrm{~nm}$ y la segunda capa es de radio $R_{3}=25 \mathrm{~nm}$ de Au. A la derecha solo el campo exterior. 

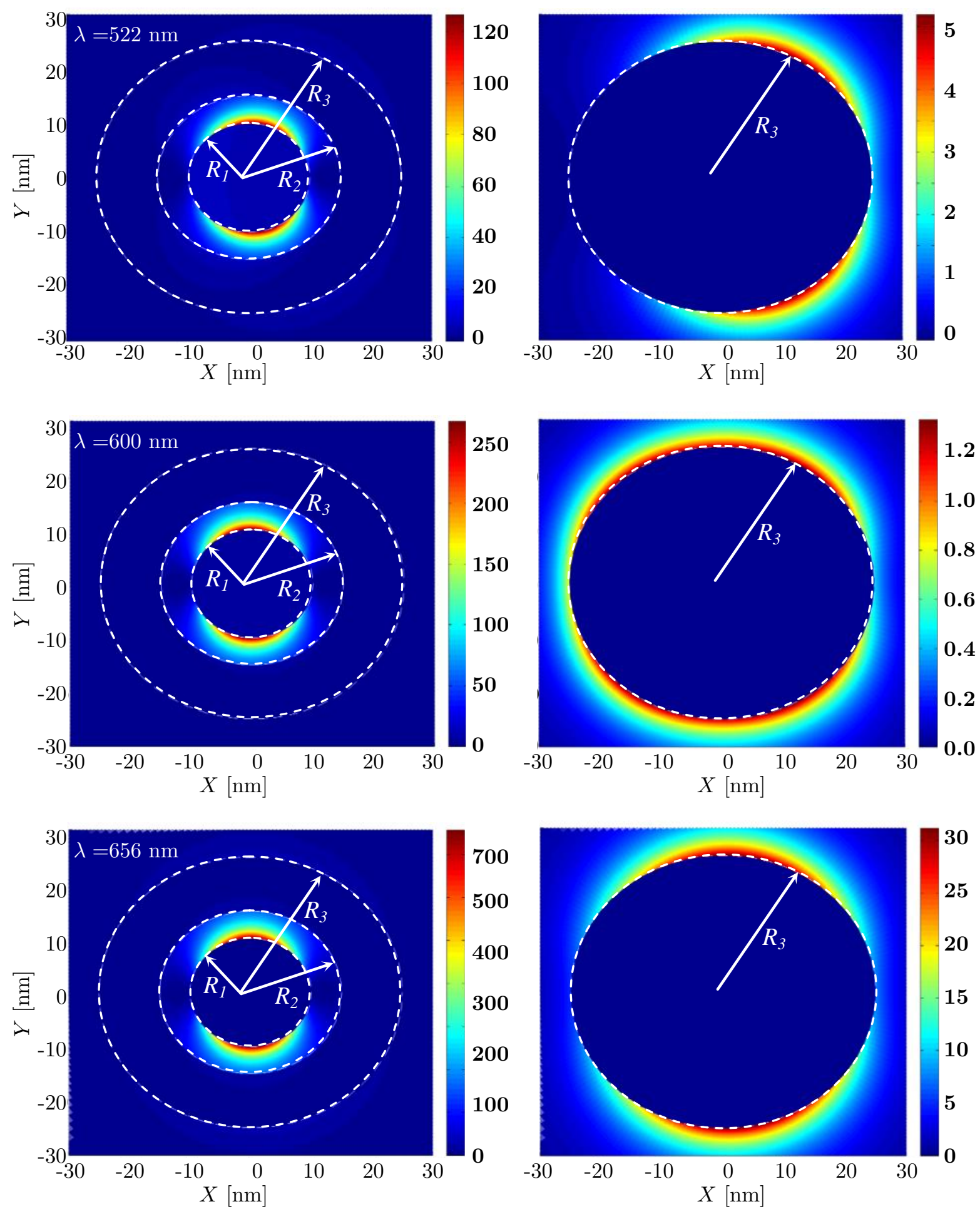

Figura 5.7: Refuerzo de campo $E / E_{0}$, para diferentes longitudes de onda $(522,600$ y $656 \mathrm{~nm}$ ) en una NP cuyo núcleo $R_{1}=10 \mathrm{~nm}$ es de Ag, la primera capa es de $\mathrm{SiO}_{2}$ de radio $R_{2}=15 \mathrm{~nm}$ y la segunda capa es de radio $R_{3}=25 \mathrm{~nm}$ de Au. A la derecha solo el campo exterior. 

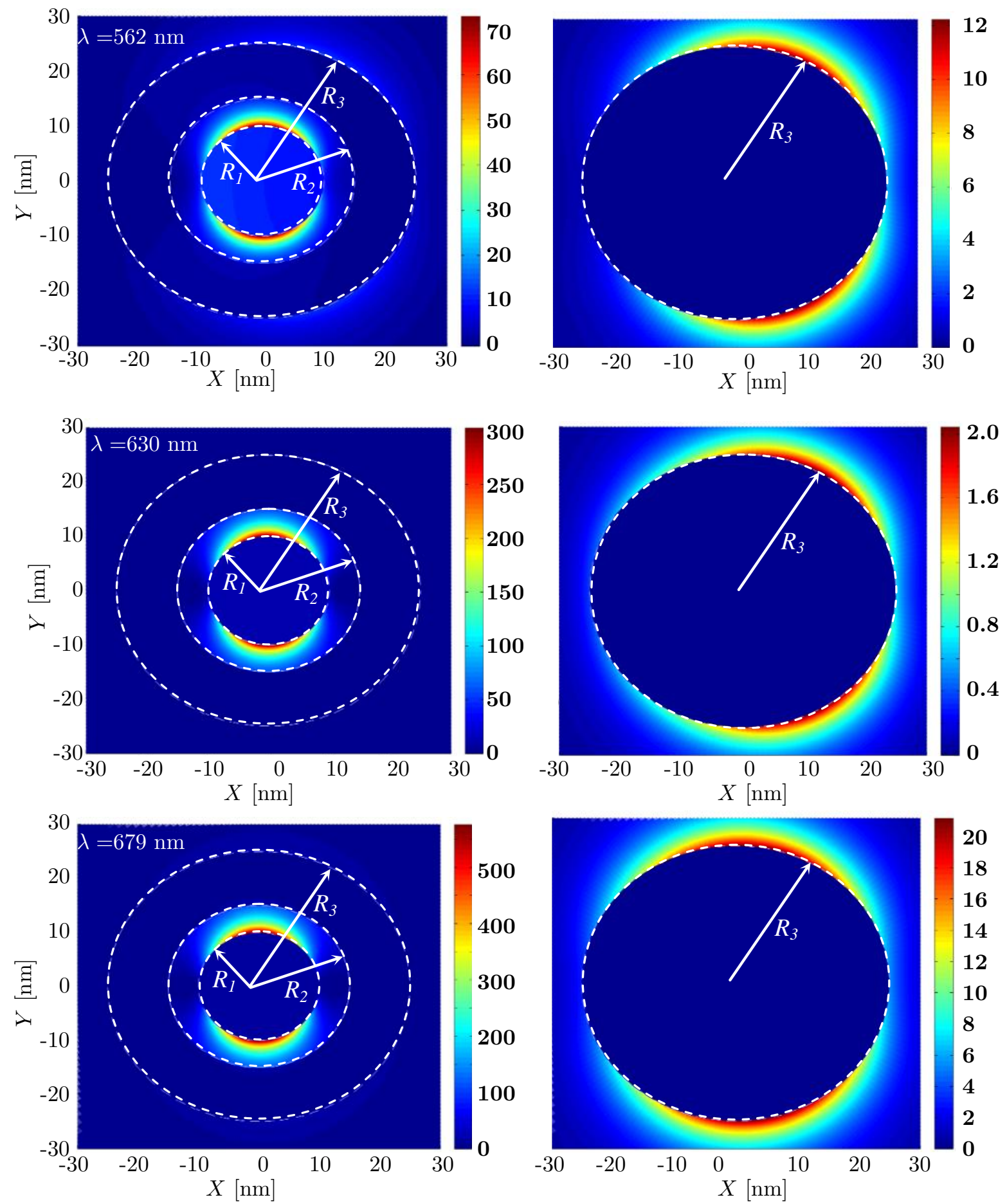

Figura 5.8: Refuerzo de campo $E / E_{0}$, para diferentes longitudes de onda $(562,630$ y $679 \mathrm{~nm}$ ) en una NP cuyo núcleo $R_{1}=10 \mathrm{~nm}$ es de $\mathrm{Cu}$, la primera capa es de $\mathrm{SiO}_{2}$ de radio $R_{2}=15 \mathrm{~nm}$ y la segunda capa es de radio $R_{3}=25 \mathrm{~nm}$ de Au. A la derecha solo el campo exterior. 

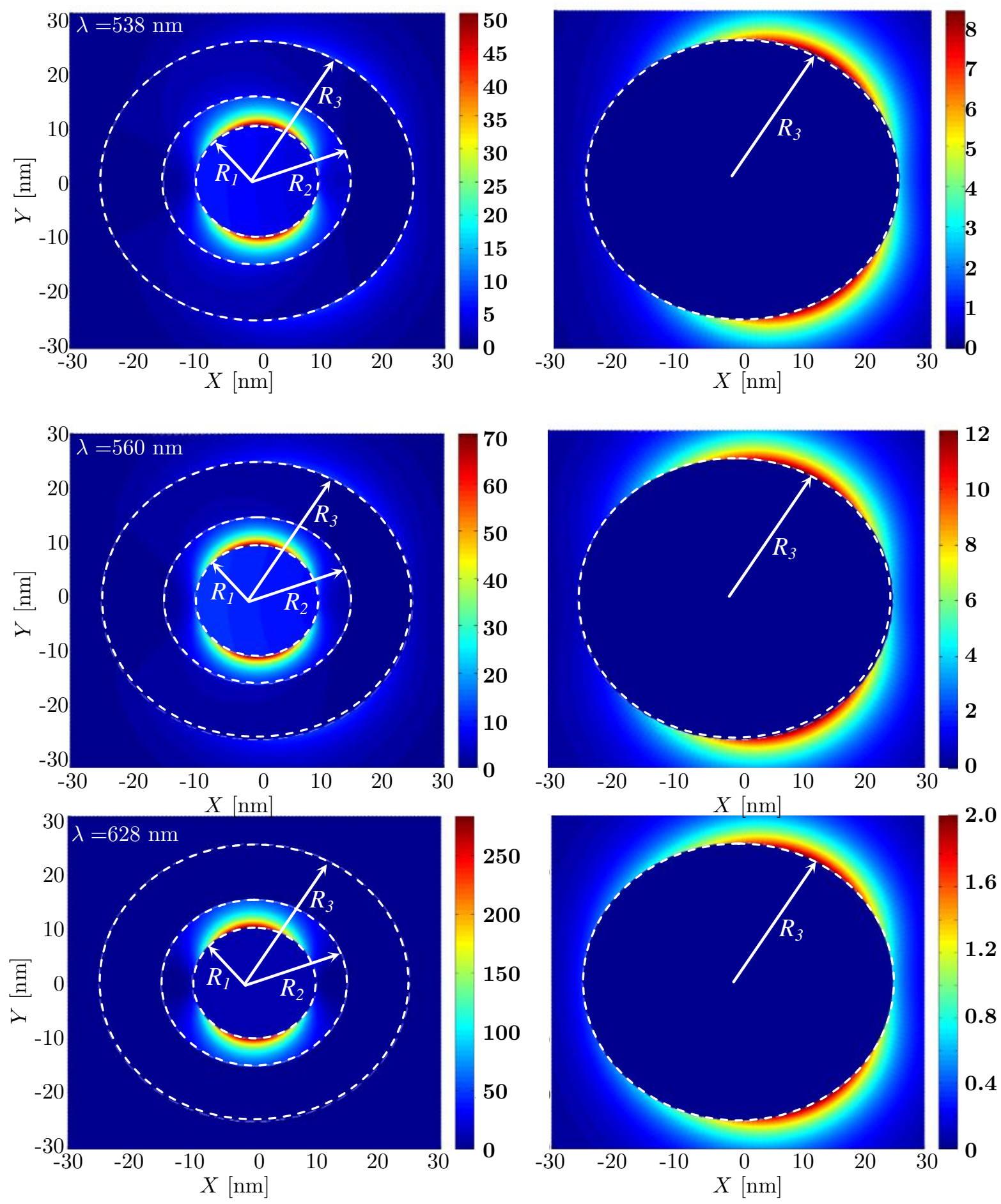

Figura 5.9: Refuerzo de campo $E / E_{0}$, para diferentes longitudes de onda $(538,560$ y $628 \mathrm{~nm}$ ) en una NP cuyo núcleo $R_{1}=10 \mathrm{~nm}$ es de Al, la primera capa es de $\mathrm{SiO}_{2}$ de radio $R_{2}=15 \mathrm{~nm}$ y la segunda capa es de radio $R_{3}=25 \mathrm{~nm}$ de $\mathrm{Au}$. A la derecha solo el campo exterior. 
Una gráfica el refuerzo de campo en el dieléctrico externo de las NPs en función de la longitud de onda para diferentes metales en el núcleo ( $\mathrm{Au}, \mathrm{Ag}, \mathrm{Cu}$ and $\mathrm{Al}$ ) se muestra en la Figura 5.10. Se observan dos picos en el refuerzo de campo, los cuales corresponden a las resonancias $\lambda_{-}^{+}$y $\lambda_{-}^{-}[\mathbf{1 3 5},[\mathbf{1 3 6}]$, definidas en la Figura $\mathbf{5 . 5}$. La resonancia de longitud de onda más larga, produce un refuerzo de campo superior cuando el núcleo de la NP es de Al y un refuerzo de campo menor cuando el núcleo es de $\mathrm{Au}$, siendo este ultimo el de mayor refuerzo de campo en la longitud de onda de resonancia más corta.

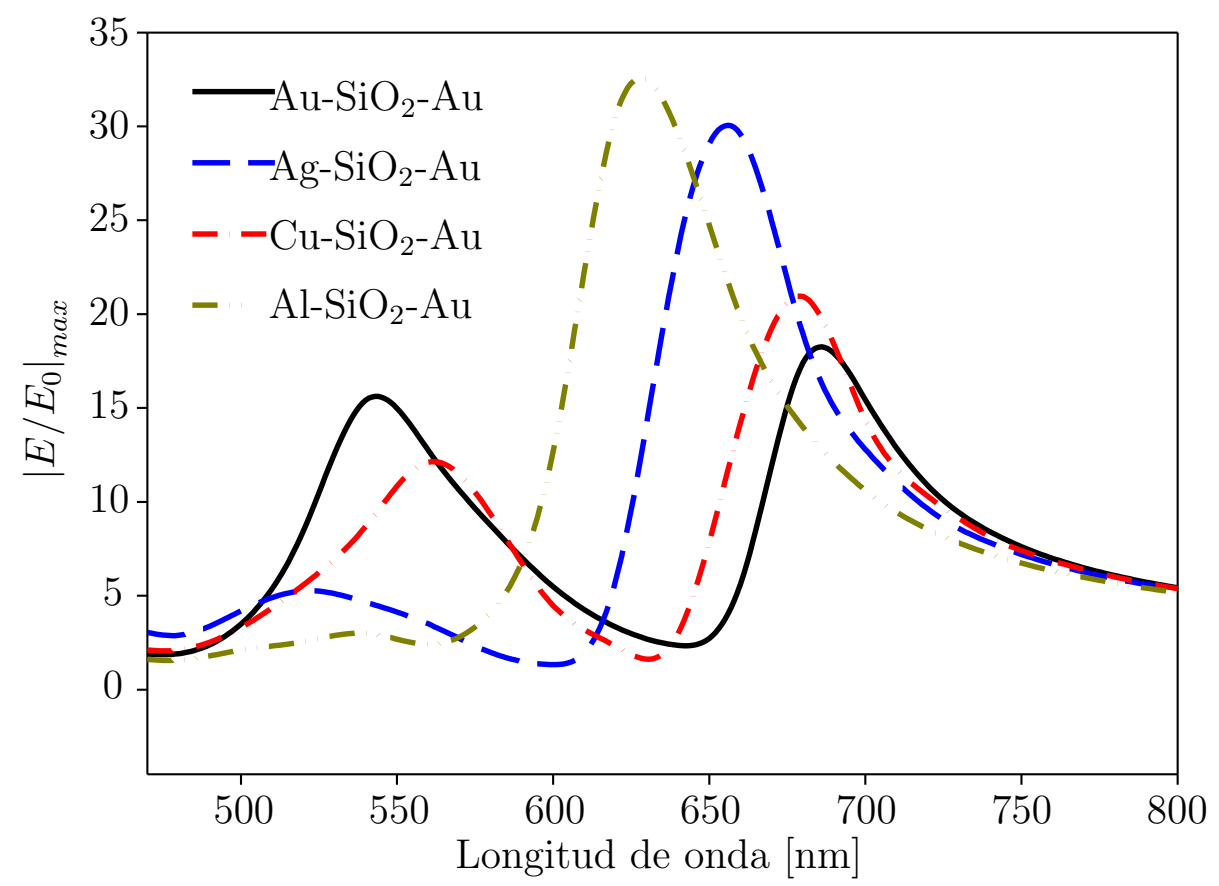

Figura 5.10: Valores máximos de las relaciones $\left|E / E_{0}\right|$ en el exterior de la nanopartícula con la configuración metal- $\mathrm{SiO}_{2}-\mathrm{Au}$, de radios 10,15 y 25 nm respectivamente.

Es importante observar que el refuerzo de campo en el dieléctrico intermetálico, presenta valores muy superiores a los obtenidos para el refuerzo de campo exterior Figura 5.11. El valor mas alto de este refuerzo se obtiene cuando el metal del núcleo es $\mathrm{Ag}$, seguidos de $\mathrm{Cu}$ y $\mathrm{Au}$ respectivamente, donde el $\mathrm{Al}$ es el que presenta un valor de refuerzo de campo menor. 
Uno de los parámetros que influye en el refuerzo de campo es la pemitividad del dieléctrico intermetálico. Para estudiar el efecto de esta permitividad, variamos su valor, en primera medida considerándolo constante con la frecuencia y en segunda medida reemplazándolo por distintos dieléctricos $\left(\mathrm{Al}_{2} \mathrm{O}_{3}\right.$, Agua, $\mathrm{SiO}_{2}$ y $\left.\mathrm{TiO}_{2}\right)$.

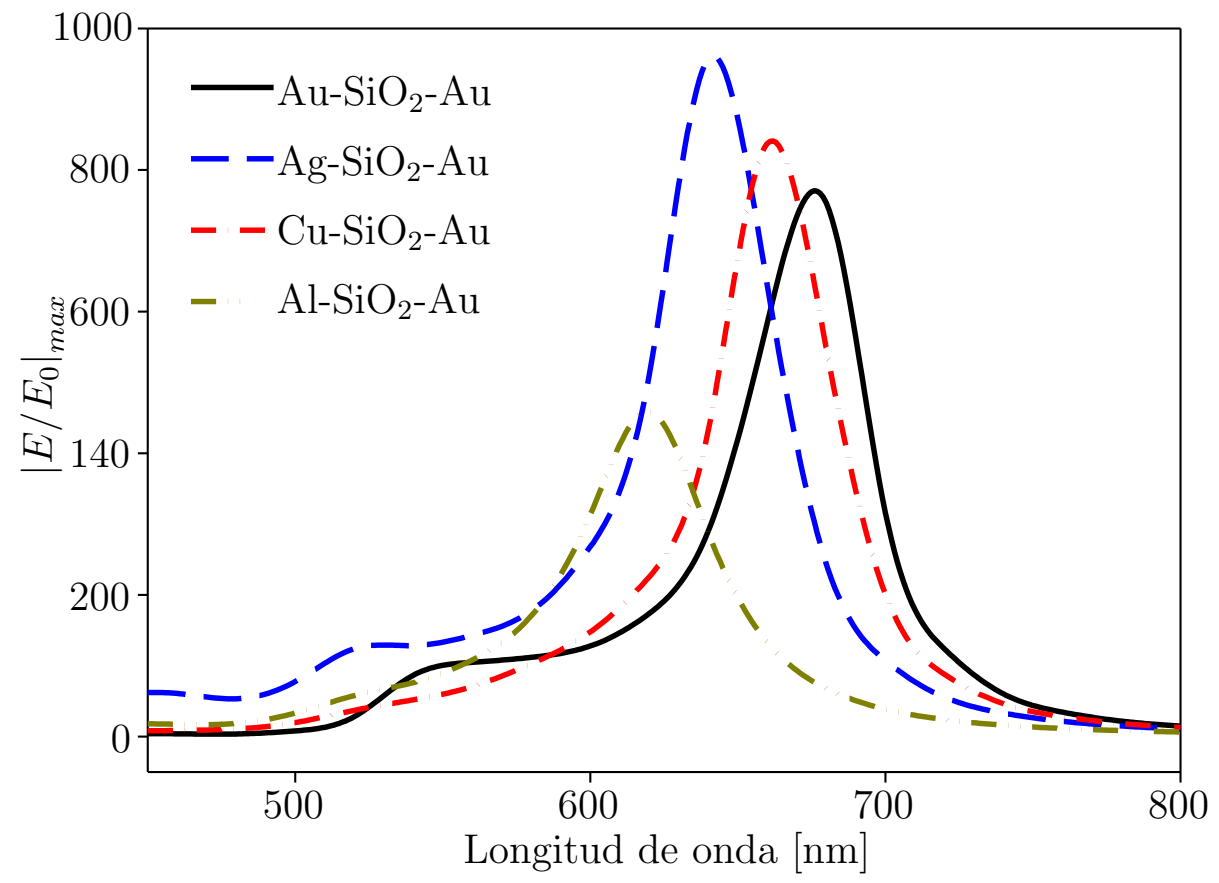

Figura 5.11: Valores máximos de las relaciones $\left|E / E_{0}\right|$ en la capa de $\mathrm{SiO}_{2}$ de la $\mathrm{NP}$ con la configuración metal- $\mathrm{SiO}_{2}$ - $\mathrm{Au}$, de radios 10,15 y $25 \mathrm{~nm}$ respectivamente.

Al incrementar el valor de la permitividad del medio dieléctrico intermetálico, Figura 5.12.a, el pico de longitud de onda más larga del refuerzo del campo externo se desplaza hacia longitudes de onda mayores y disminuye su amplitud. Adicionalmente el pico de resonancia del refuerzo de campo correspondiente a la longitud de onda más corta crece en amplitud y se desplaza hacia longitudes de onda más largas pero su desplazamiento es menor en proporción al presentado por el pico de resonancia de longitud de onda más larga. El desplazamiento de los picos de resonancia a longitudes de onda más largas, se debe al crecimiento de la longitud de onda de la resonancia del núcleo $\lambda_{s}$ (Figura 5.5), por el incremento de la permitividad que lo rodea. Este desplazamiento de $\lambda_{s}$ mejora el acoplamiento que da lugar a la resonancia $\lambda_{-}^{+}$, lo que justifica el crecimiento del pico de resonancia correspondiente a la longitud de onda 
más corta. Adicionalmente se desfavorece el acoplamiento que da lugar a la resonancia $\lambda_{-}^{-}$, con lo cual disminuye el valor del refuerzo de campo del pico de longitud de onda mas larga.
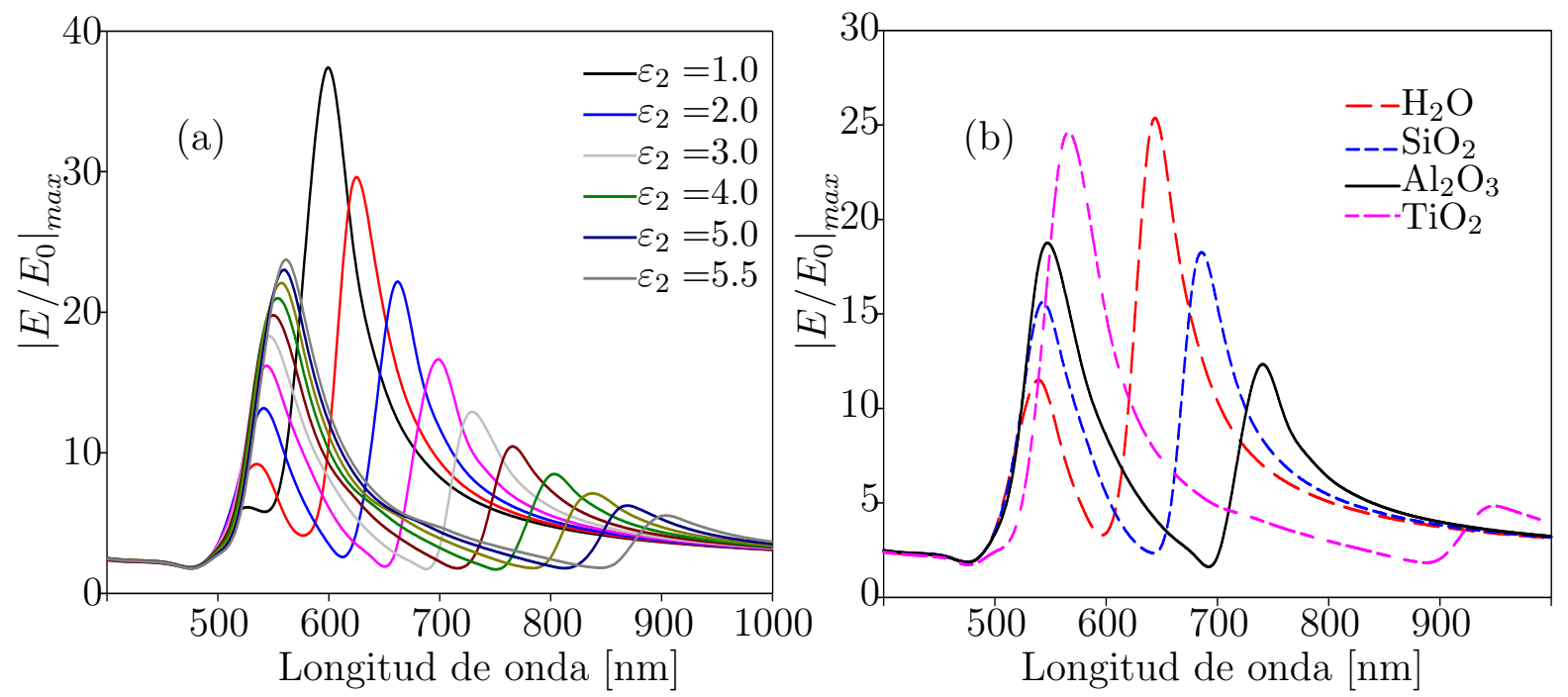

Figura 5.12: Valores máximos de las relaciones $\left|E / E_{0}\right|$ en el exterior de la NP con la configuración metal- $\mathrm{SiO}_{2}-\mathrm{Au}$, de radios 10,15 y $25 \mathrm{~nm}$ respectivamente.

En diversas aplicaciones se requiere el máximo valor del campo externo, en cuyo caso es conveniente utilizar el dieléctrico adecuado como se muestra en la Figura 5.12.b, donde el mayor valor del refuerzo de campo eléctrico se obtiene utilizando como dieléctrico el agua, que produce mayores refuerzos de campo que el obtenido con los otros dieléctricos $\left(\mathrm{Al}_{2} \mathrm{O}_{3}, \mathrm{SiO}_{2}\right.$ y el $\left.\mathrm{TiO}_{2}\right)$. Para la misma configuración de tamaños y para el pico de longitud de onda más corta, el $\mathrm{TiO}_{2}$ produce un máximo refuerzo de campo.

Un efecto distinto ocurre en el refuerzo de campo en el dieléctrico intermetálico donde al incrementar los valores de $\varepsilon_{2}$, primero se presenta un incremento del refuerzo de campo hasta cierto valor y luego una disminución del mismo, como se observa en la Figura 5.13,a. El valor mas alto del refuerzo de campo correspondiente a 850.8 ocurre cuando $\varepsilon_{2}=2.8$. En el caso de dieléctricos que dependen de la frecuencia la máxima amplitud del refuerzo de campo presenta intensidades y ubicaciones diferentes para cada uno de los dieléctricos. Como se muestra en la Figura 5.13.b, para el caso 
del $\mathrm{H}_{2} \mathrm{O}$ el máximo se encuentra en longitudes de onda más cortas mientras que para el caso del $\mathrm{TiO}_{2}$ se encuentra en longitudes de onda más largas cercano a $920 \mathrm{~nm}$ de manera que eligiendo el dieléctrico adecuado se logra una sintonia.
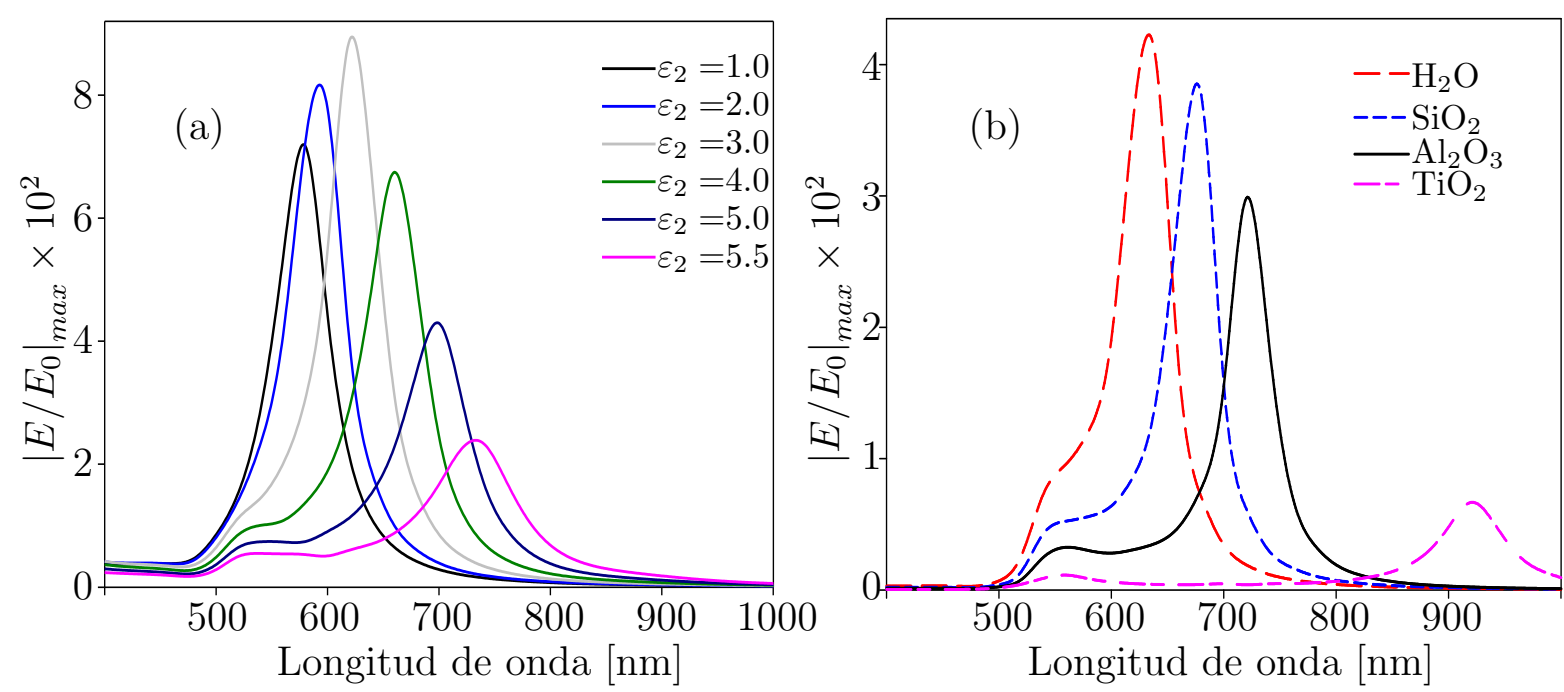

Figura 5.13: Valores máximos de las relaciones $\left|E / E_{0}\right|$ en el interior de la NP con la configuración metal- $\mathrm{SiO}_{2}-\mathrm{Au}$, de radios 10,15 y $25 \mathrm{~nm}$ respectivamente.

Al incrementar el valor de la permitividad $\varepsilon_{2}$ del dieléctrico intermetálico, todos los valores de longitudes de onda a las cuales ocurren las resonancias del núcleo $\lambda_{s}$ y las resonancias de la nano-cubierta $\lambda_{ \pm}$(Figura 5.5), se desplazan hacia longitudes de onda más largas. Lo cual a su vez provoca que las longitudes de onda $\lambda_{-}^{ \pm}$se desplacen hacia longitudes de onda más largas.

La longitud de onda de resonancia del núcleo presenta un desplazamiento con la permitividad del dieléctrico intermetálico mayor que el de las correspondientes a la nano-cubierta al estar más influenciada por la permitividad del dieléctrico intermetálico. Este desplazamiento mayor de $\lambda_{s}$ ocasiona que se acerque más a $\lambda_{-}$y se aleje mas de $\lambda_{+}$.

El resultado final de esto es un incremento en el acoplamiento que da lugar a $\lambda_{-}^{-}$ (aumento de la amplitud del pico) debido a la mejor sintonía y un decrecimiento en el acoplamiento que da lugar a $\lambda_{-}^{+}$(disminución de la amplitud del pico). 


\subsection{Extinción óptica de un arreglo lineal de nanopartículas}

Cuando las NPs se encuentran en arreglos espacialmente ordenados, sus características plasmónicas difieren de aquellas observadas en NPs aisladas. Las propiedades ópticas de estos ensambles los hacen atractivos para aplicaciones en sensores plasmónicos nanoestructurados [139], cristales fotónicos 140-150 y otros. El caso más simple corresponde a un arreglo lineal de NPs pequeñas de diámetro $2 R$ [151-155], separadas una distancia $D$ entre ellas. El hecho de tener las NPs posicionadas a lo largo de una recta rompe la simetría esférica que posee cada una de las nanoesferas y se genera una birrefringencia geométrica, por lo cual es de esperar que la extinción óptica sea diferente dependiendo de la dirección de incidencia de la OEM. Además, también es de esperar que las resonancias plasmónicas dependan de la longitud total de la nanoestructura.

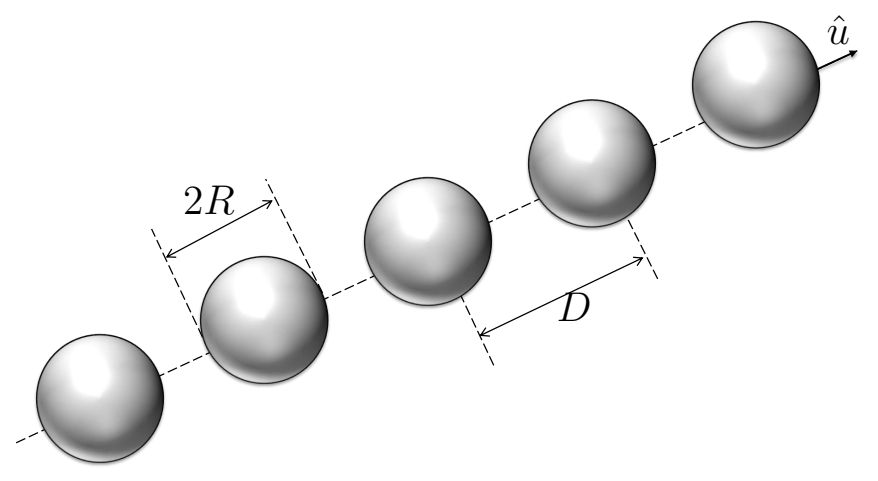

Figura 5.14: Esquema correspondiente a un arreglo líneal de NPs pequeñas de diámetro $2 R$, separadas una distancia $D$ entre ellas.

En este caso, no es posible utilizar teoría de Mie para calcular espectros de extinción, ya que aquélla no tiene en cuenta la posible interacción entre NPs. De este modo, una estrategia consiste en utilizar métodos numéricos que permita superar esta dificultad. En esta tesis usaremos la llamada aproximación de discretización por dipolos (Discrete Dipole Approximation, DDA), que supone que las NPs son muy pequeñas en comparación con la longitud de onda y por lo tanto pueden ser consideradas como 
dipolos.

En nuestro problema, supondremos que las NPs son de Ag y se encuentran alineadas sobre una recta cuyo vector de dirección se define como $\hat{u}$, como se muestra en la Figura 5.14 .

Si suponemos que el arreglo y el campo aplicado se encuentran en la dirección del eje $X$ y el vector de propagación $\mathbf{k}$ se encuentra en la dirección $Z$, un incremento del número de NPs (y por ende la longitud del cilindro simulado), desplaza la longitud de onda a la cual se producen las resonancias longitudinales del cilindro hacia el rojo, como se observa en la Figura $\mathbf{5 . 1 5}$.

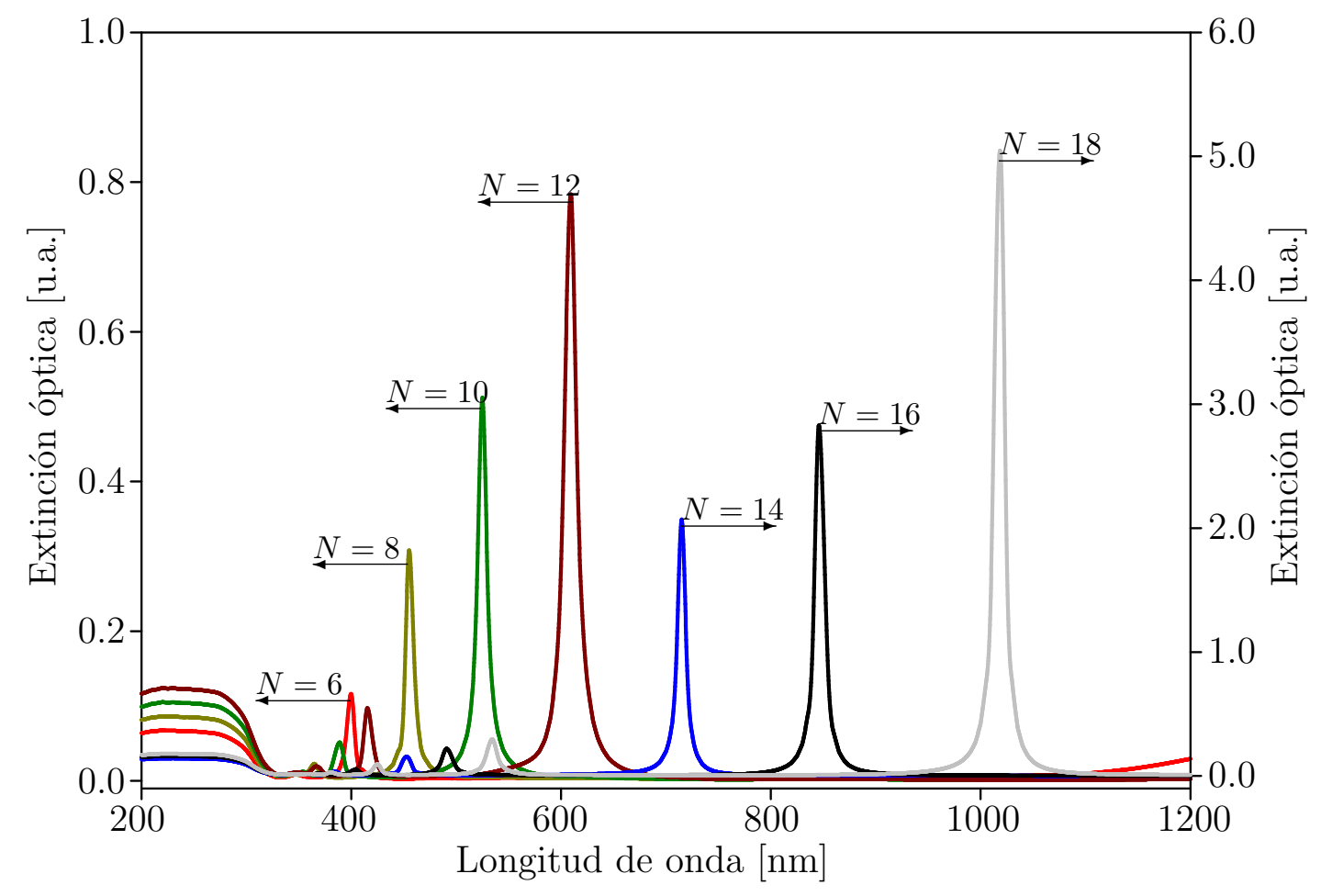

Figura 5.15: Espectro de extinción para un arreglo de NPs esféricas de Ag de $3 \mathrm{~nm}$ de radio, separados una distancia $D=6 \mathrm{~nm}$, el arreglo posee vector $\mathbf{u}$ en la dirección del eje $X$, el campo aplicado se encuentra en la dirección $X$ y el vector de propagación $\mathbf{k}$, se encuentra en la dirección $Z$.

Si se modifica la dirección del campo incidente a la dirección $[1,1,0]$, los espectros de extinción óptica se modifican como se muestra en la Figura 5.16. En ésta se observa una frecuencia de resonancia en aproximadamente $350 \mathrm{~nm}$ correspondiente a la respuesta 
del arreglo a la componente del campo eléctrico perpendicular al eje del cilindro, que no cambia su ubicación debido a que el diámetro del cilindro no cambia, aunque se agreguen NPs. El incremento en la amplitud de este pico es causada por el incremento del número de NPs. Adicionalmente se observa otra resonancia que se desplaza hacia longitudes de onda más largas al incrementar la longitud del cilindro (aumento del número de NPs). Esta resonancia corresponde a la respuesta de la componente del campo paralela al eje del cilindro. Estos resultados coinciden con los que se obtienen para cilindros macizos utilizando el formalismo de Mie en coordenadas cilíndricas [13].

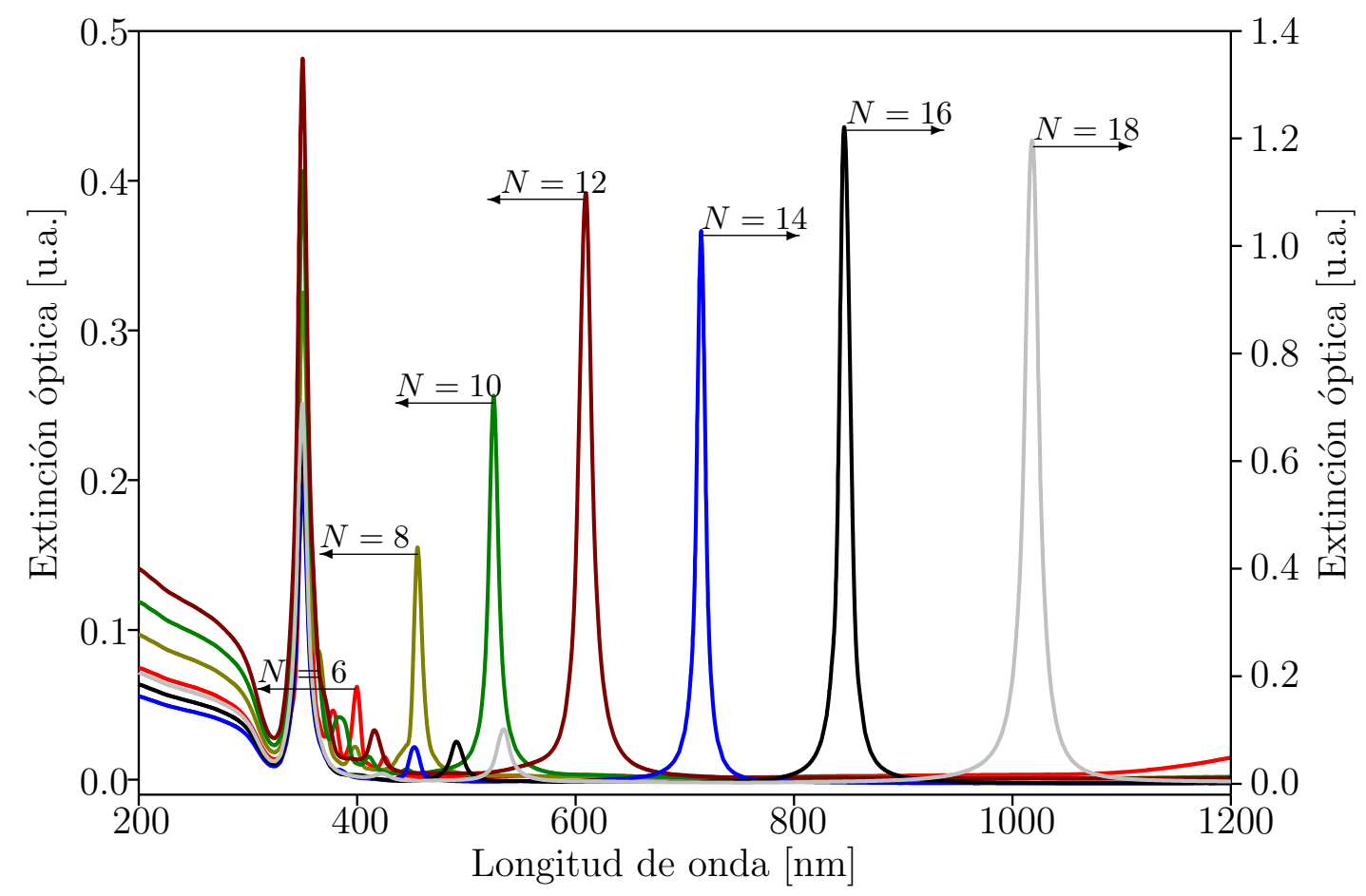

Figura 5.16: Espectro de extinción para un arreglo de NPs esféricas de Ag de $3 \mathrm{~nm}$ de radio, separados una distancia entre centros de $6 \mathrm{~nm}$, el arreglo posee vector $\mathbf{u}$ en la dirección del eje $X$, el campo aplicado se encuentra en la dirección $[1,1,0]$ y el vector de propagación $\mathbf{k}$, se encuentra en la dirección $Z$. $N=6-20$

En la mayor parte de las aplicaciones, las NPs con las cuales se forman arreglos no son de tamaño pequeño como para ser asimiladas a dipolos simples . En este caso, consideramos que cada una de ellas está compuesta por un conjunto de dipolos. Si tomamos una de las NPs como referencia, los momentos dipolares de los dipolos que 
conforman las otras partículas son iguales al de la partícula de referencia, multiplicados por un desfasaje. En este caso se considera un sistema compuesto por $M$ NPs idénticas ubicadas en las posiciones $\mathbf{r}_{k}$, donde $k=1 \cdots M$, las cuales están compuestas por $N$ dipolos. Estos se encuentran en las posiciones $\mathbf{r}_{i}$ con respecto al vector $\mathbf{r}_{k}$ de la NP y presentan momentos dipolares $\mathbf{p}_{i}$, donde $i=1 \cdots N$. El momento dipolar del $i$-esimo dipolo de la $k$-esima NP se escribe como [153], [156], 157, 158], 159]:

$$
\mathbf{p}_{i, k}=\mathbf{p}_{i, 1} e^{i \mathbf{k} \cdot \mathbf{r}_{k}}
$$

donde $\mathbf{p}_{j, 1}$ es el momento dipolar del $j$-esimo dipolo de la NP ubicada en el sistema de referencia. Con todas estas consideraciones el campo resultante en un dipolo de la NP de referencia es el del campo incidente mas las contribuciones de todos los otros dipolos. Esto se puede analizar como $M$ matrices de interacción donde la primera de estas matrices representa la interacción de los dipolos de la NP de referencia, en la que diagonal es cero (ver Apéndice C). La expresión para el momento del $j$-esimo dipolo de la NP de referencia se convierte en:

$$
\begin{aligned}
\frac{\mathbf{p}_{j, 1}}{\alpha_{j}} & =\mathbf{E}_{i n c, j, 1}+\sum_{i \neq j}^{N} A_{i j, 1} \mathbf{p}_{i, 1}+\sum_{k=2}^{M} \sum_{i=1}^{N} A_{i j, k} \mathbf{p}_{i, 1} e^{i \mathbf{k} \cdot \mathbf{r}_{k}} \\
& =\mathbf{E}_{i n c, j, 1}+\sum_{k=1}^{M} \sum_{i=1}^{N} A_{i j, k}\left(1-\delta_{k} \delta_{j}\right) \mathbf{p}_{i, 1} e^{i \mathbf{k} \cdot \mathbf{r}_{k}\left(1-\delta_{k}\right)}
\end{aligned}
$$

La solución del sistema de ecuaciones $\mathbf{5 . 2}$, produce los momentos dipolares de cada uno de los dipolos de la NP ubicada en el sistema de referencia. En este sistema $A_{i j, k}$ es igual a la representada por la ecuación C.7 donde $\mathbf{r}=\mathbf{r}_{i}-\mathbf{r}_{j}-\mathbf{r}_{k}$, en el sistema escogido para $k=1, \mathbf{r}_{k}=0$.

Cuando se tiene un número elevado de NPs grandes esféricas, como ocurre en un cristal fotónico, el número de dipolos que se deben utilizar para reproducir el sistema es elevado. Esta situación requiere un tiempo de cómputo que, en algunos casos resulta prohibitivo para usuarios sin acceso sistemas de cómputo de alto rendimiento. Además, las NPs utilizadas para generación de cristales fotónios sintonizables suelen ser del 
tipo núcleo-cubierta. Para solucionar este problema, se ideó una estrategia que consiste en determinar la polarizabilidad efectiva de las NPs a partir de la teoría de Mie. Si igualamos las eficiencias de extinción y absorción obtenidas por la teoría de Mie para NPs núcleo-cubierta a las definiciones de la aproximación dipolar para NPs sin cubierta, obtendremos una polarizabilidad equivalente para las NPs núcleo-cubierta, de las cuales sus partes real e imaginarias son:

$$
\begin{gathered}
\alpha_{e f f}^{I}=\frac{\pi b^{2}}{k} Q_{\text {ext }}^{M i e} \\
\alpha_{e f f}^{R}=\sqrt{\frac{6 \pi^{2} b^{2}}{k^{4}} Q_{s c a}^{M i e}-\left(\alpha_{e f f}^{I}\right)^{2}}
\end{gathered}
$$

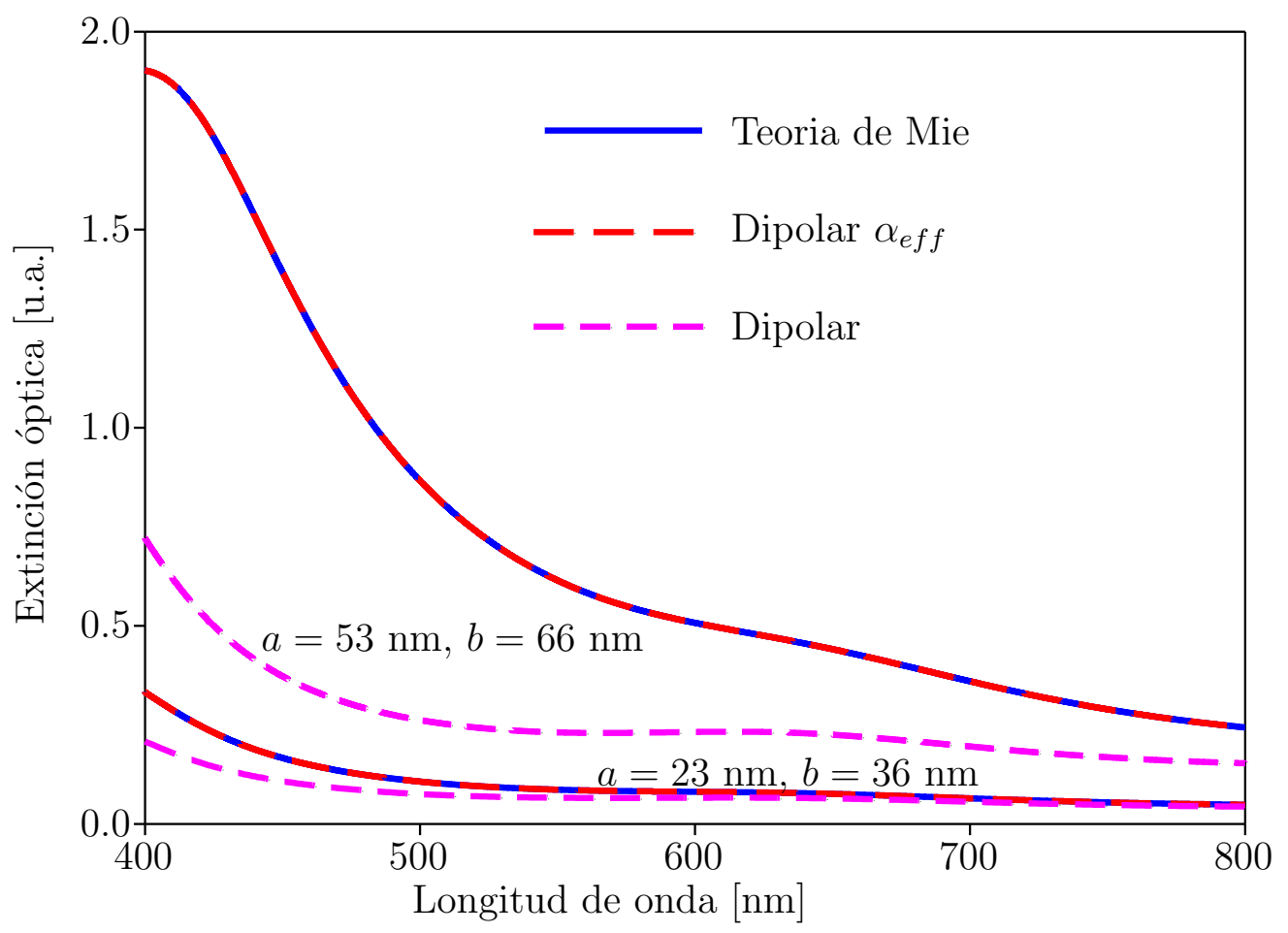

Figura 5.17: Espectros de extinción de $\mathrm{NPs}$ de $\mathrm{Fe}_{3} \mathrm{O}_{4}-\mathrm{SiO}_{2}$, en línea continua azul utilizando la teoría de Mie, en segmentos rojos utilizando la polarizabilidad efectiva en aproximación dipolar y en segmentos cortos magenta utilizando la aproximación dipolar, para los radios $a$ y $b$ especificados.

En la Figura 5.17 se muestran espectros de extinción de $\mathrm{NPs}$ de $\mathrm{Fe}_{3} \mathrm{O}_{4}-\mathrm{SiO}_{2}$ 
en azul utilizando la teoría de Mie y el segmentos rojos utilizando la aproximación dipolar incluyendo la polarizabilidad equivalente definida por las ecuaciones $5 \mathbf{5 . 3}$ y 5.4) para distintos radios de las NPs y en segmentos cortos magentas la aproximación dipolar utilizando la polarizabilidad definida en el Apéndice B. Para radios pequeños $b<15 \mathrm{~nm}$ la aproximación dipolar presenta una buena descripción de la extinción óptica, aunque no resulta una buena aproximación para tamaños mayores. En cambio, es posible utilizar la aproximación dipolar para tamaños grandes introduciendo la polarizabilidad efectiva.

Adicionalmente, en la Figura 5.18, se comparan las polarizabilidades definidas en el Apéndice B y en las ecuaciones (5.3 y [5.4), donde se observan claramente las diferencias ocasionadas al no utilizar teoría de Mie para determinar la polarizabilidad de NPs de tamaños grandes (aproximadamente $b>15 \mathrm{~nm}$ ). Esta diferencia es mucho mas evidente en la parte imaginaria de la polarizabilidad.

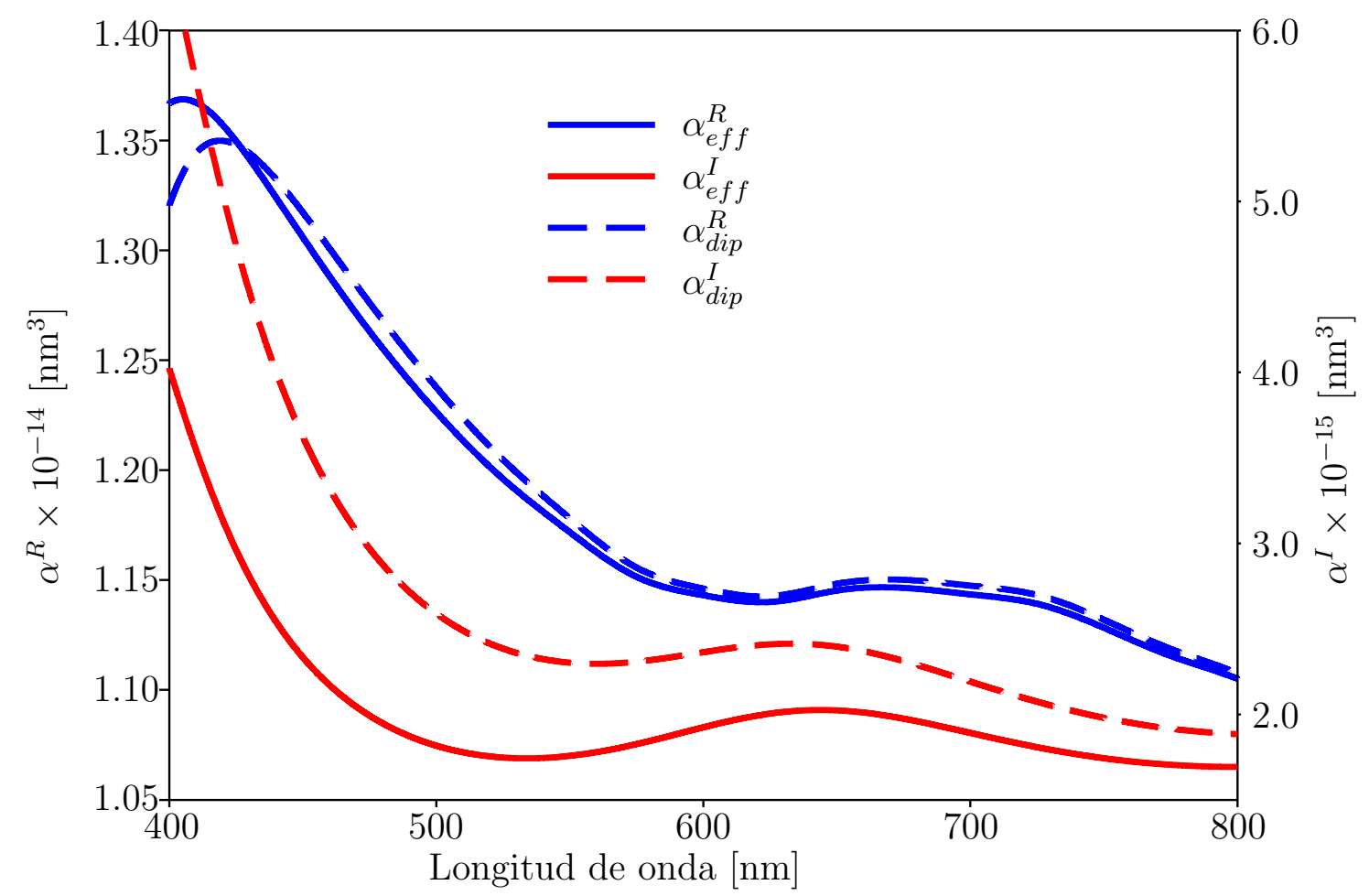

Figura 5.18: Partes real e imaginarias de la polarizabilidad dipolar y efectiva en NPs de $\mathrm{Fe}_{3} \mathrm{O}_{4}-\mathrm{SiO}_{2}$, con radios $a=23 \mathrm{~nm}$ y $b=36 \mathrm{~nm}$ 
Esta polarizabilidad efectiva se puede utilizar en el código DDA para calcular la respuesta óptica de arreglos de NPs núcleo-cubierta de diámetros superiores a 30 nm y describiendo las ondas EM en los modos TE y TM. En la Figura $\mathbf{5 . 1 9}$ se muestran los resultados de la extinción óptica de un arreglo de 100 NPs de $\mathrm{Fe}_{3} \mathrm{O}_{4}-\mathrm{SiO}_{2}$ de $53 \mathrm{~nm}-66 \mathrm{~nm}$ con una separación de $100 \mathrm{~nm}$. La contribución del modo TM es mucho menor en comparación con el pico de extinción obtenido utilizando el modo TE que se encuentra en una longitud de onda de $417 \mathrm{~nm}$.

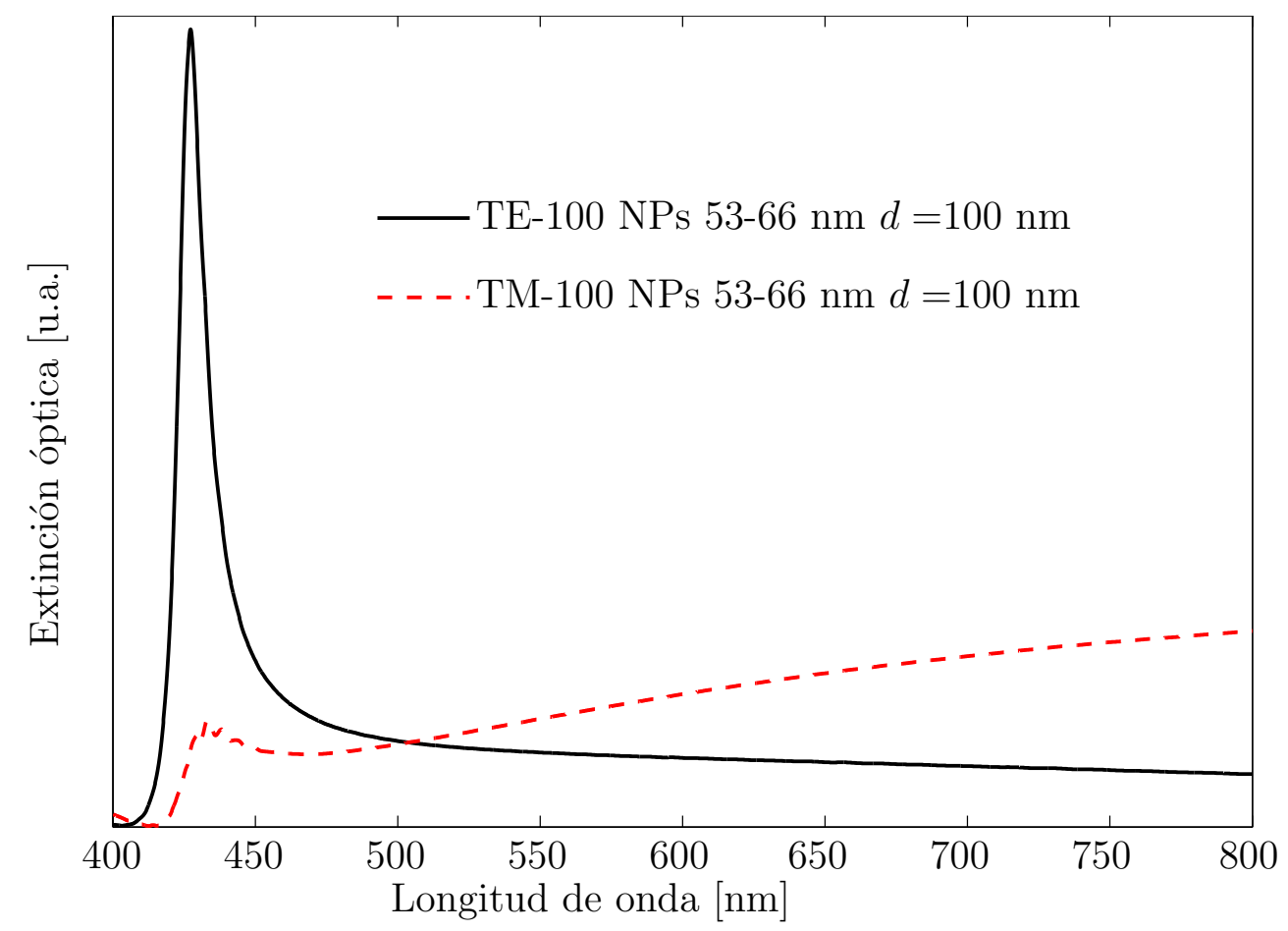

Figura 5.19: Extinción para los modos TE y TM en un arreglo de 100 NPs de $\mathrm{Fe}_{3} \mathrm{O}_{4}-\mathrm{SiO}_{2}$ y la separación entre NPs es $d=100 \mathrm{~nm}$.

En la Figura 5.20 se muestra el efecto del número de NPs en la extinción cuando la incidencia de la onda es del tipo TE. Al incrementar el número de NPs, se produce un incremento de la extinción óptica por un cambio en la densidad de NPs consideradas, mientras que la ubicación del pico de extinción no cambia. Esto se debe a que su ubicación depende fundamentalmente del índice de refracción efectivo de la $\mathrm{Np}$, del índice de refracción del medio en el cual se encuentran las NPs, del tamaño de las NPs y de la separación de las mismas. 


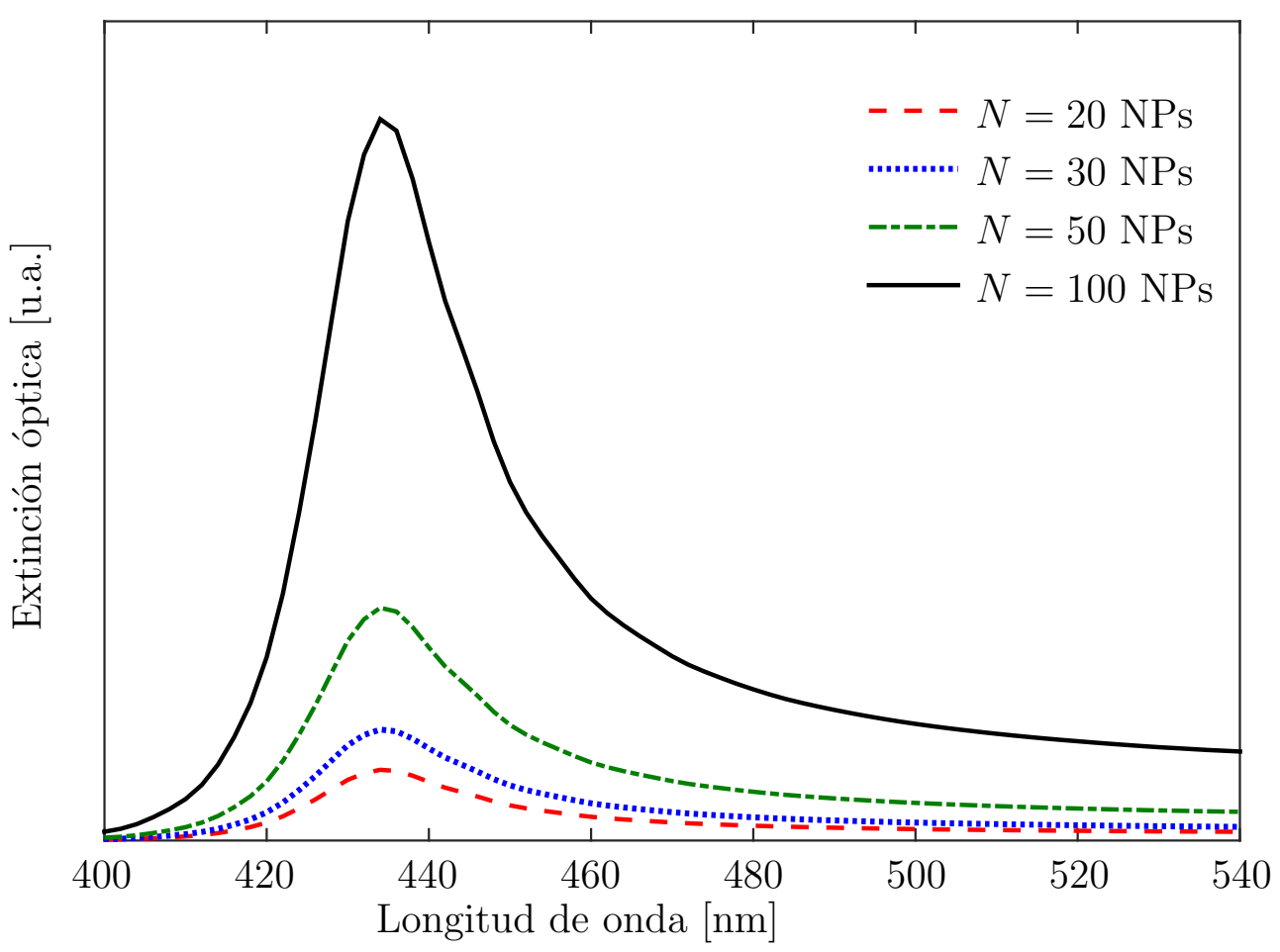

Figura 5.20: Influencia en la extinción óptica del número de NPs. Las NPs son de $\mathrm{Fe}_{3} \mathrm{O}_{4}-\mathrm{SiO}_{2}$ y la separación entre NPs es $d=100 \mathrm{~nm}$.

En la Figura 5.21 se muestra la evolución del pico de extinción para 100 NPs de $\mathrm{Fe}_{3} \mathrm{O}_{4}-\mathrm{SiO}_{2}$ para distintos valores del radio del núcleo (a) y separadas $120 \mathrm{~nm}$, donde el espesor de la cubierta de $\mathrm{SiO}_{2}$ se mantiene constante en $13 \mathrm{~nm}$. Se observa al aumentar el radio de la NP se produce un corrimiento del pico de extinción hacia longitudes de onda mas largas. Se muestra en segmentos rojos el ajuste del pico de extinción como una función del radio interior de las NPs. La ecuación correspondiente al ajuste es $\lambda_{p}=-0,0081 a^{2}+4,9182 a+213,2349$, lo que muestra una respuesta muy cercana a una recta.

Un resultado importante que surge de esta figura es que existe un tamaño mínimo de NPs para el cual el pico de extinción se encuentra en la región del espectro visible. En particular, para un espesor de cubierta de $13 \mathrm{~nm}$ y cuando el radio interior de la NP es aproximadamente $40 \mathrm{~nm}$, la longitud de onda del pico de extinción se encuentra cercana al inicio del visible en $400 \mathrm{~nm}$. Por tanto, el tamaño mínimo de las NPs debe ser $53 \mathrm{~nm}$ para que su pico de extinción se ubique en el visible. 


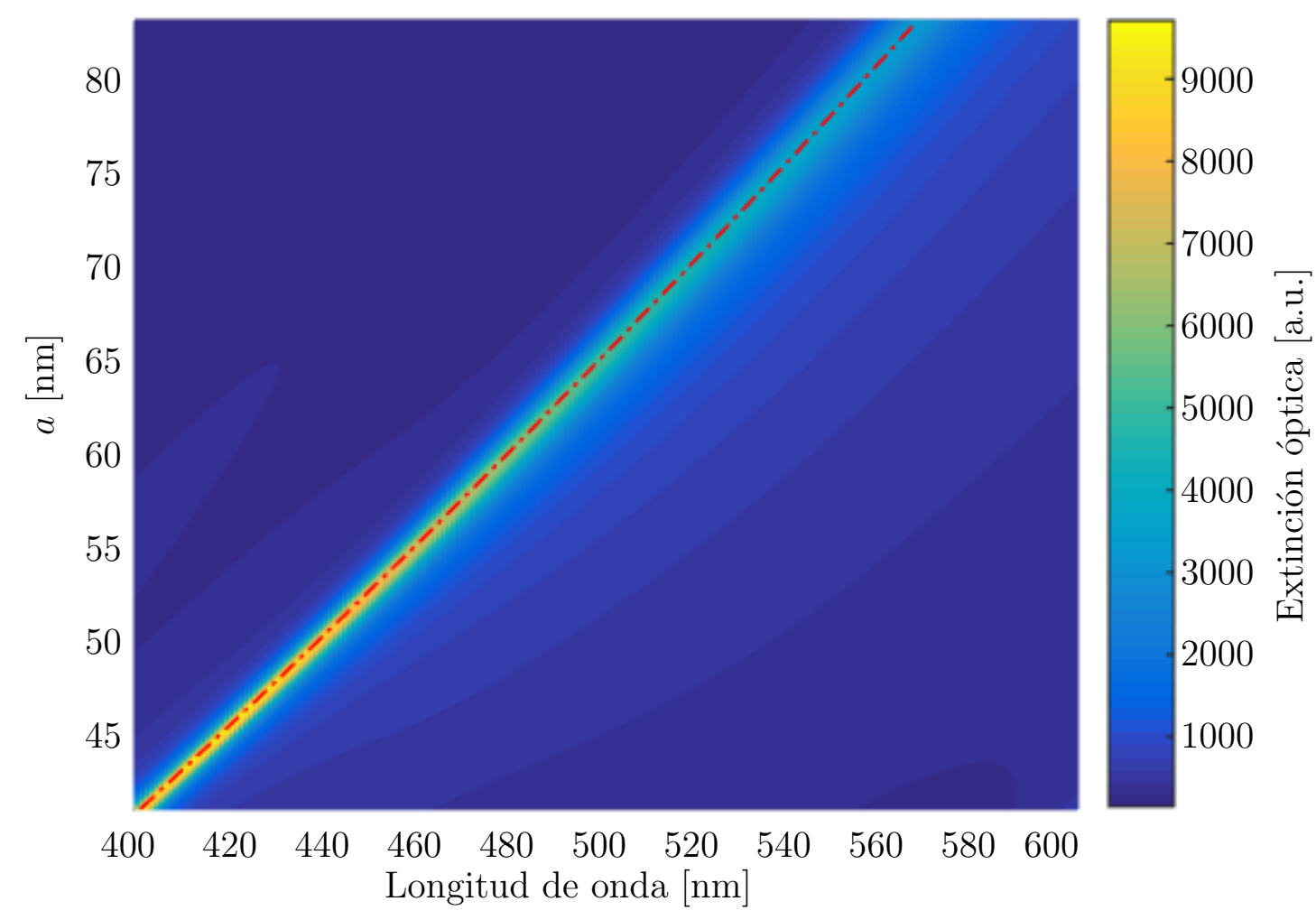

Figura 5.21: Extinción para el modo TE en un arreglo de $100 \mathrm{NPs}$ de $\mathrm{Fe}_{3} \mathrm{O}_{4}-\mathrm{SiO}_{2}$ y la separación entre NPs es $d=120 \mathrm{~nm}$.

En la Figura 5.22 se cambia el material de la cubierta $\left(\mathrm{SiO}_{2}, \mathrm{Al}_{2} \mathrm{O}_{3}, \mathrm{TiO}_{2}\right.$, Si y $\mathrm{Au}$ ) para NPs de $53 \mathrm{~nm}$ de núcleo y $33 \mathrm{~nm}$ de espesor (estos valores se tomaron para mostrar claramente el efecto que tiene sobre el espectro de extinción tomar distintas cubiertas). Se observa que el pico de extinción óptica cambia su ubicación dependiendo del material de la cubierta, obteniéndose corrimientos hacia el rojo más importantes cuando la cubierta es de Au. Lo que implica que en el caso de NPs con cubierta de Au se requieren NPs más pequeñas para lograr un cristal fotónico en el visible. 


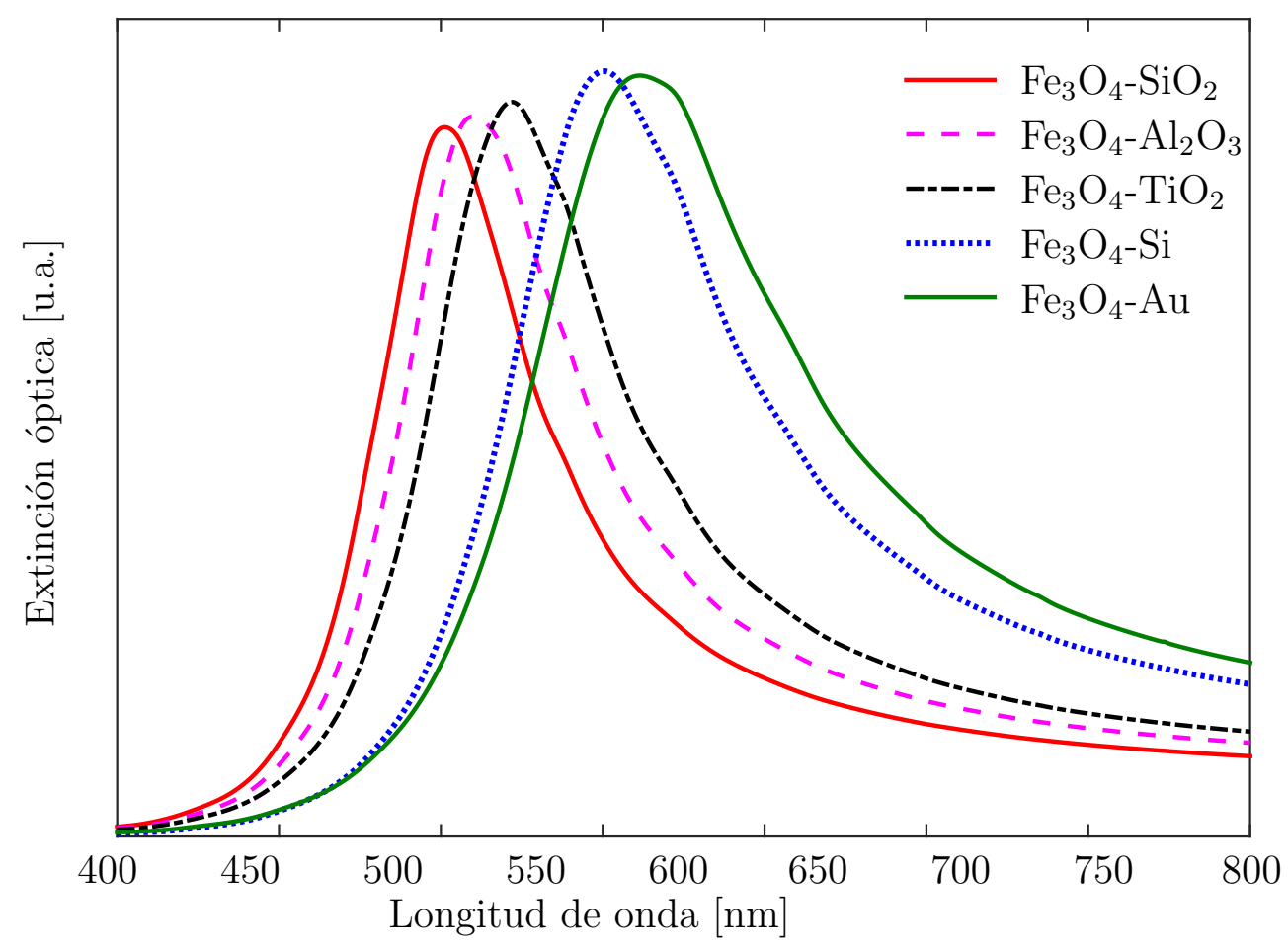

Figura 5.22: Influencia del del material de la cubierta en un arreglo de NPs con núcleo de $\mathrm{Fe}_{3} \mathrm{O}_{4}$, separación $d=120 \mathrm{~nm}, a=53 \mathrm{~nm}$ y $b=86 \mathrm{~nm}$ en la extinción. El número de NPs es 100.

$\mathrm{Hu}$ et al. [148]), propone un cristal fotónico sintonizable en el visible formado por NPs de $\mathrm{Fe}_{3} \mathrm{O}_{4}-\mathrm{SiO}_{2}$. La sintonia se logra por la aplicación de un gradiente de campo magnético externo, acercando a la suspensión un imán que modifica la distancia entre las NPs, cambiando la ubicación del pico de extinción. Al acercar el imán el gradiente de campo es más intenso y por ende las NPs se encuentran más cerca una de otras.

Con la finalidad de reproducir el efecto del campo sobre el cristal fotónico en la Figura 5.23, se muestra la evolución del pico de extinción, para distintos valores de separación entre las NPs que conforman el arreglo. Se muestra en segmentos rojos el ajuste del pico de extinción como una función de la separación $d$ de las NPs cuando $a=63 \mathrm{~nm}$ y $b=76 \mathrm{~nm}$. La ecuación correspondiente al ajuste es $\lambda_{p}=-0,0008 D^{2}+1,8823 D+67,3488$, lo que muestra una respuesta muy cercana a una recta. 


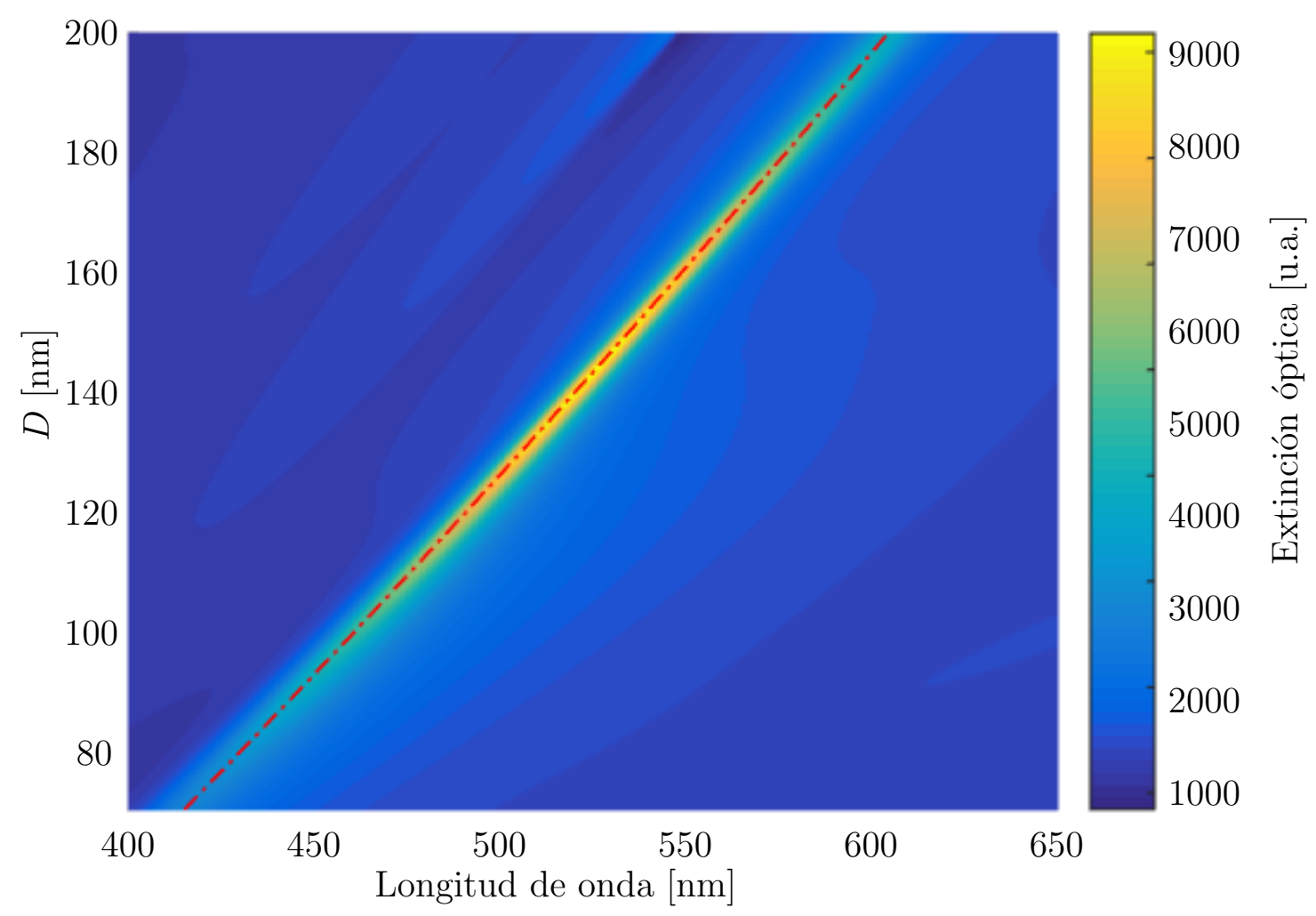

Figura 5.23: Influencia de la separación de las NPs en un arreglo de NPs con núcleo de $\mathrm{Fe}_{3} \mathrm{O}_{4}, a=63 \mathrm{~nm}$ y $b=76 \mathrm{~nm}$ en la extinción. El número de NPs es 100 .

En la Figura 5.23, la separación entre las NPs es la misma para todas. Pero existen condiciones experimentales en las que el gradiente de campo magnético es no uniforme. Este gradiente provoca que las NPs que se encuentran más lejos de la fuente experimenten una mayor separación entre NPs que las que se encuentran mas cerca de la fuente de campo. Para analizar este fenómeno consideraremos que el espaciado de las NPs es mayor a medida que estas se encuentra más lejos de la fuente de campo, como se ilustra en la Figura 5.24. Este efecto lo controlamos con el factor $\Delta d$, que en el caso de ser cero todas las NPs se encuentran separadas $120 \mathrm{~nm}$, y por ejemplo, cuando es $15 \mathrm{~nm}$, la primera se encuentra separada de la segunda en $120 \mathrm{~nm}$ y la última de la penúltima en $135 \mathrm{~nm}$. Entre estos dos extremos la diferencia de separación entre NPs es líneal. $\mathrm{Al}$ incrementar el valor del parámetro $\Delta d$, se produce un corrimiento hacia longitudes de onda más largas y un ensanchamiento del pico de extinción óptica. 


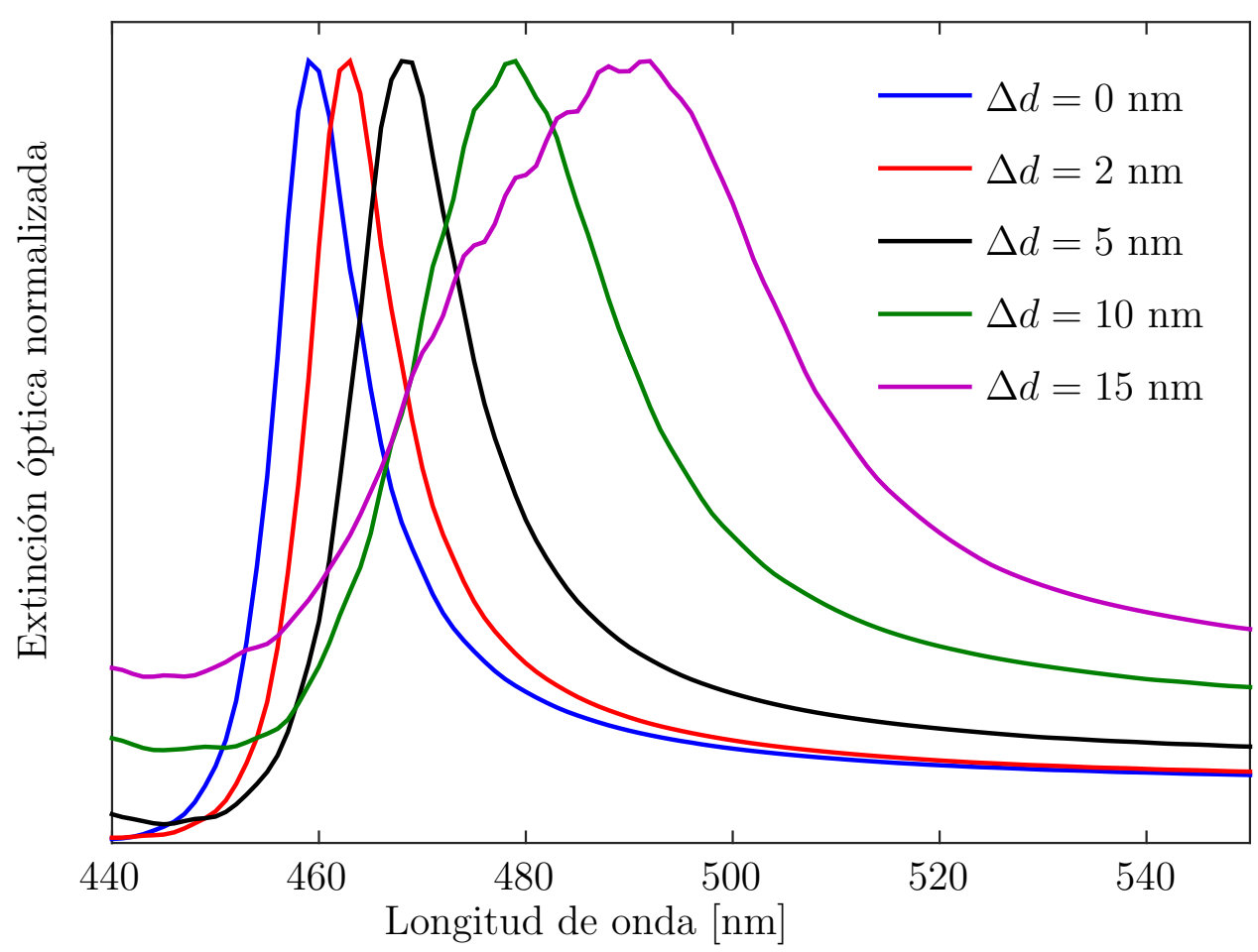

Figura 5.24: Influencia del gradiente de campo en un arreglo de NPs con núcleo de $\mathrm{Fe}_{3} \mathrm{O}_{4}$, separación inicial $d_{0}=120 \mathrm{~nm}, a=53 \mathrm{~nm}$ y $b=66 \mathrm{~nm}$ en la extinción. El número de NPs es 100.

Hasta el momento hemos considerado que todas las NPs son del mismo radio, es decir no presentan dispersión de tamaños. Pero en la mayor parte de los experimentos las NPs presentan dispersión de tamaños. Por tanto es importante considerar la dispersión como un factor en la extinción óptica del cristal fotónico.

En la Figura 5.25, se muestra cómo cambia el pico de extinción óptica del arreglo de $100 \mathrm{NPs}$ de $\mathrm{Fe}_{3} \mathrm{O}_{4}-\mathrm{SiO}_{2}(53-66 \mathrm{~nm})$ separadas $120 \mathrm{~nm}$, para diferentes distribuciones de tamaño. Esta es de tipo log-normal, con $a_{m}=53 \mathrm{~nm}$ y $\sigma$ variable entre 0.01 y 0.4 . El pico de extinción se ensancha y desplaza hacia longitudes de onda más largas con el incremento de la dispersión de las NPs. 


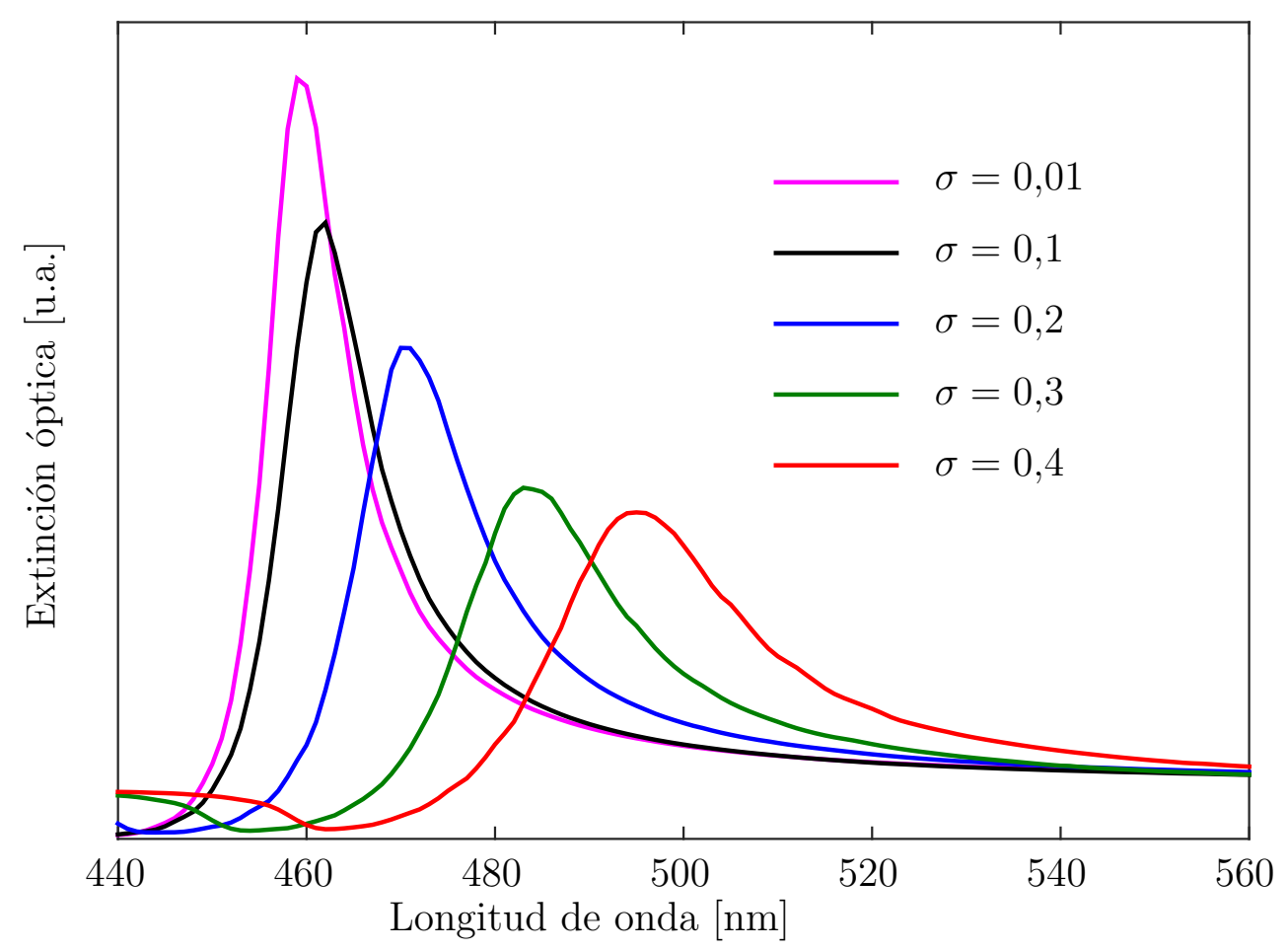

Figura 5.25: Influencia de la dispersión de tamaños en un arreglo de NPs con núcleo de $\mathrm{Fe}_{3} \mathrm{O}_{4}$, separación $d=120 \mathrm{~nm}, a=53 \mathrm{~nm}$ y $b=66 \mathrm{~nm}$ en la extinción.

Medidas experimentales de la reflectividad para un arreglo de NPs de $\mathrm{Fe}_{3} \mathrm{O}_{4}-\mathrm{SiO}_{2}$ de radios 53-66nm realizadas por $\mathrm{Hu}$ et al. [148], aplicando un gradiente de campo magnético producido por un imán a diversas distancias, muestran diferentes posiciones para el pico de extinción. Estos resultados experimentales se muestran en círculos y líneas de segmentos en la Figura 5.26. Una primera aproximación para ajustar los resultados experimentales consiste en buscar las distancias entre NPs, que produzca la misma ubicación del pico experimental, considerando que las NPs no presentan dispersión de tamaños y que se encuentran equiespaciadas. Es fácil notar que si bien se ajusta la ubicación de los picos, el ancho de los mismos es mucho mayor en el caso experimental. Este fenómeno puede ser causado por la dispersión de tamaños de las NPs o por la variación del gradiente de campo magnético. Por tanto para obtener un ajuste más cercano a las medidas experimentales se deben considerar ambos aspectos. 


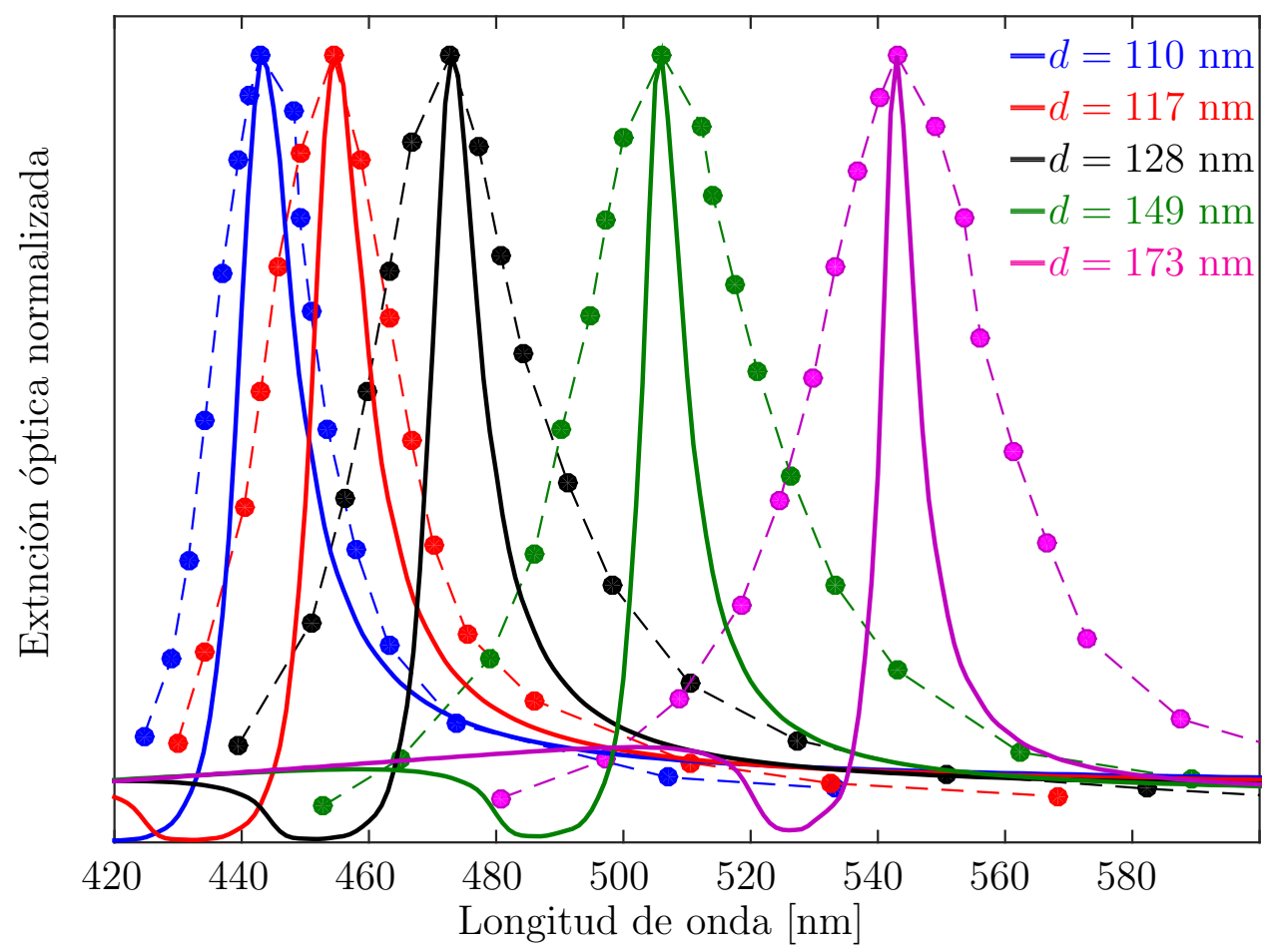

Figura 5.26: Ajuste de valores experimentales sin distribución de tamaños en las NPs y sin una variación del gradiente de campo magnético.

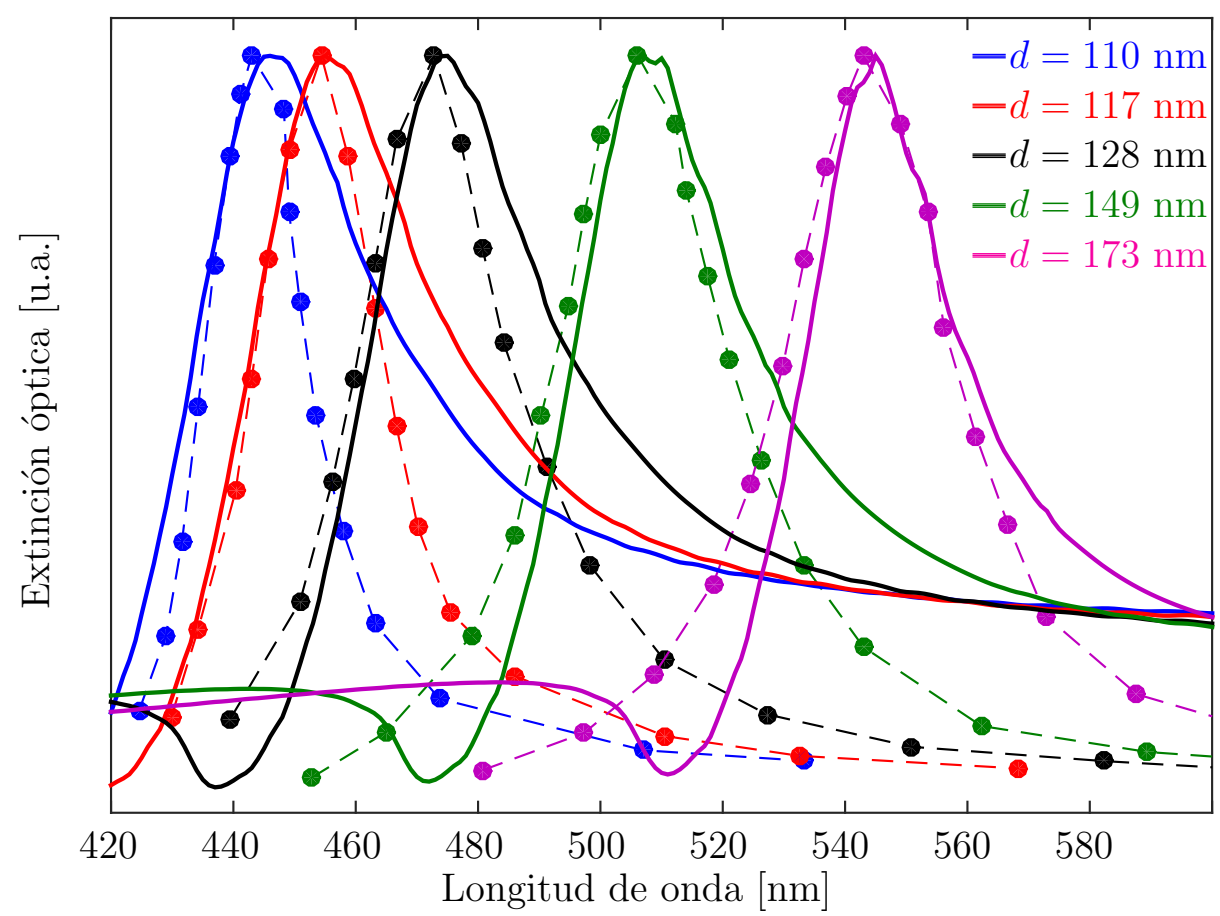

Figura 5.27: Ajuste de valores experimentales con una distribución de tamaños en las NPs correspondiente a $\sigma=0.25$. 
Si suponemos una distribución de tamaños y ajustamos la ubicación de los picos de tal forma que el ancho del pico correspondiente a la posición más lejana del imán sea la adecuada obtenemos una distribución de tamaños correspondiente a $\sigma=0.5$. Con esta distribución de tamaños los resultados son los ilustrados en la Figura $\mathbf{5 . 2 7}$.

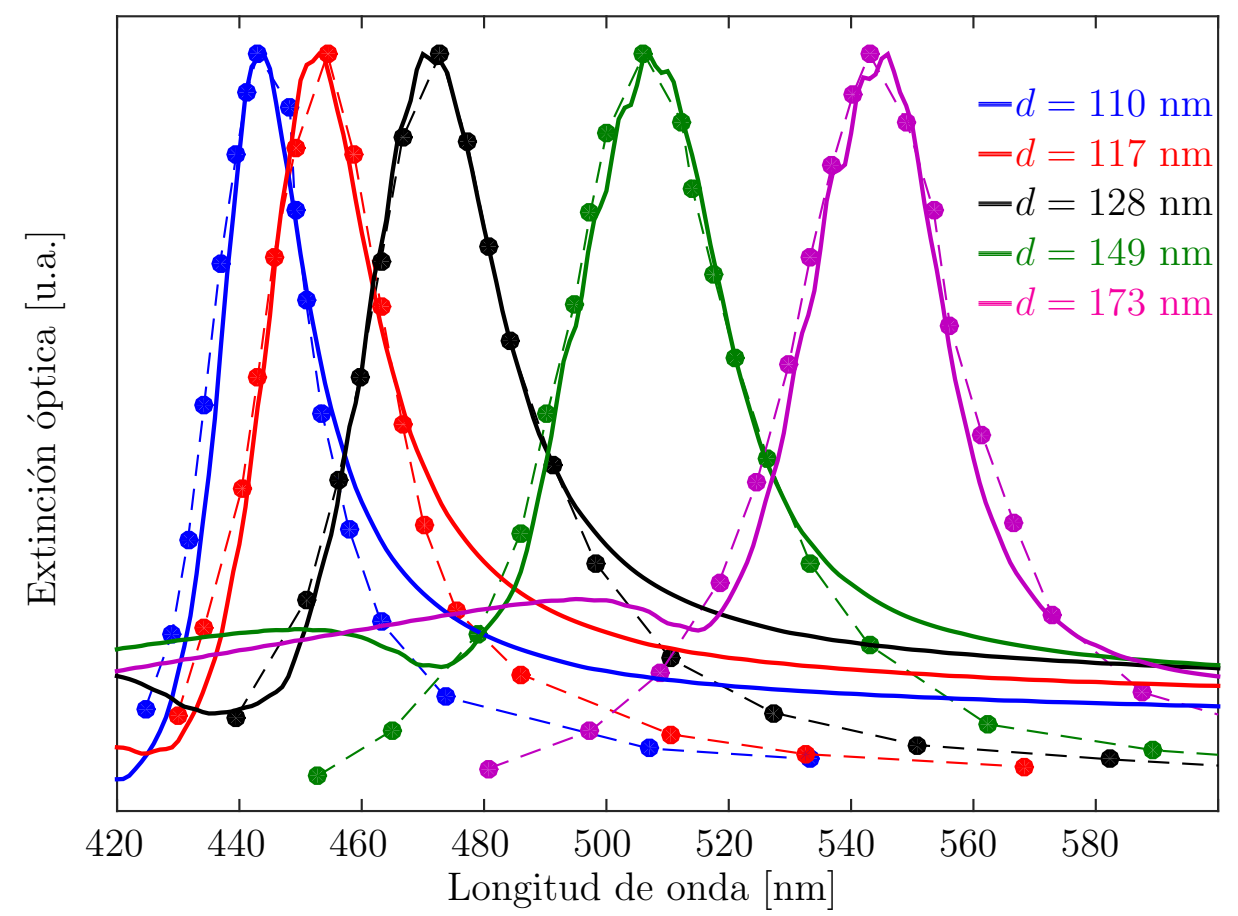

Figura 5.28: Ajuste de valores experimentales con una distribución de tamaños en las NPs correspondiente a $\sigma=0.25$ y un gradiente espacial de campo magnético equivalente a $\Delta d=11 \mathrm{~nm}$.

Utilizando distribuciones de tamaño angostas ( $\sigma$ más pequeño), se ajustan los picos correspondientes al iman mas cercanos a la muestra, mientras que utilizando distribuciones de tamaño mas anchas ( $\sigma$ más grandes) se ajustan los picos correspondientes al iman mas lejos de la muestra, pero la muestra tiene una sola distribución de tamaños. En este caso se debe considerar ademas de la distribución de tamaños, la variación del gradiente del campo magnético. Considerando una variación del gradiente de campo magnético y una distribución de tamaños de las NPs, los ajustes son los ilustrados en la Figura 5.28. En esta figura se observa una buena concordancia entre los valores experimentales y los valores ajustados. La distribución de tamaños obtenida corresponde a $\sigma=0.2$ y el valor del párametro $\Delta d=11 \mathrm{~nm}$, que en términos 
de gradiente de separación de NPs corresponde a 1.04nm por cada $\mu \mathrm{m}$.

Resumiendo, el Capítulo 5 describe el refuerzo de campo de NPs esféricas con núcleo y 2 cubiertas, para lo cual en este trabajo de tesis se extendió el desarrollo matemático de la teoría de Mie al caso de NPs con 3 condiciones de contorno, determinando las expresiones de los coeficientes de los campos en cada una de las regiones. Finalmente utilizando la aproximación de dipolos discretos, conjuntamente con teoría de Mie, se determina la sección eficaz de extinción para la caracterización de cristales fotónicos formados por arreglos de NPs de magnetita con una capa de dióxido de silicio. 


\section{Capítulo 6-
Conclusiones Generales}

Este trabajo de tesis se ha centrado en el estudio y caracterización de las propiedades plasmónicas y ópticas de nanoestructuras esféricas metálicas simples y de multicapas de tipo metal-dieléctrico-metal, incluyendo especies con núcleo magnético. Algunas de las nanoestructuras estudiadas fueron fabricadas por ablación láser de femtosegundos en nuestro laboratorio ( $\mathrm{Al}, \mathrm{Ag}, \mathrm{Cu}$ simples y con una capa de óxido), mientras que otras $\left(\mathrm{Au}-\mathrm{Fe}_{3} \mathrm{O}_{4}\right)$ se obtuvieron por síntesis química en laboratorios que colaboran con nuestra línea de trabajo. Las nanoestructuras de $\mathrm{Na}$ en haluros alcalinos presentadas en el capítulo 4, fueron obtenidas por nuestros colegas del CIOp Dres. JorgeTocho y Alvaro Carrera utilizando la técnica de precipitado térmico en sólidos cristalinos.

Se ha desarrollado la formulación teórica necesaria para interpretar y reproducir los espectros experimentales de extinción óptica de NPs esféricas generadas por los métodos antes mencionados, utilizando teoría de Mie e incluyendo la modificación de la función dieléctrica por tamaño. Dicha modificación se realizó a partir del modelo clásico de Drude, corrigiendo primeramente la constante de amortiguamiento del modelo para tener en cuenta las colisiones de los electrones de conducción con los bordes de la NP y considerando luego las transiciones interbanda para dar cuenta de los efectos de los electrones ligados. Fueron determinados los parámetros de Drude $\omega_{p}$ y $\gamma$ utilizando un novedoso método que parte de los valores en volumen (bulk) de la función dieléctrica para intervalos de longitud de onda extendidos, que cubren desde el UV hasta el lejano IR. Este método fue aplicado primeramente a metales nobles y luego se extendió a numerosos metales de transición (y otros), de modo de completar unos 15 elementos de 
la tabla periódica. Se observó que en el caso de los electrones libres se deben realizar correcciones para radios de NPs inferiores a 50nm y en el caso de la contribución de los electrones ligados, para radios inferiores a $2 \mathrm{~nm}$.

Luego de realizar un estudio de la teoría de Mie para NPs de núcleo desnudo y núcleo-cubierta, se extiende la teoría a NPs con dos cubiertas. Se desarrollaron programas computacionales en plataforma MATLAB para el cálculo de distribuciones espaciales de campo, eficiencias y secciones eficaces de extinción, scattering y absorción para los tipos de NPs antes mencionados. Con éstos, se mostró que no considerar la corrección de la función dieléctrica por tamaño para tamaños inferiores a $20 \mathrm{~nm}$ presenta errores importantes en la predicción de los espectros de extinción. También se encontró que la aplicación de la aproximación electrostática no solo depende del tamaño de las NPs (pequeñas) sino también del material. Por ejemplo, en el caso del Au, la aproximación electrostática es válida para radios menores a $10 \mathrm{~nm}$, mientras que en el caso del Al lo es para radios inferiores a $5 \mathrm{~nm}$.

Con el uso de los programas de cálculo basados en teoría de Mie y subrutinas adicionales, se determinó la distribución log-normal de tamaño de NPs de Au, Al, $\mathrm{Fe}_{3} \mathrm{O}_{4}$ y $\mathrm{Fe}_{3} \mathrm{O}_{4}-\mathrm{Au}$, donde la comparación con técnicas tradicionales (TEM y AFM) mostró la eficacia del método de espectroscopía de extinción óptica, que en el caso de las NPs de $\mathrm{Al}$ permitió predecir las especies presentes en la solución (Al, $\mathrm{Al}_{-} \mathrm{Al}_{2} \mathrm{O}_{3}$, $\mathrm{Al}_{2} \mathrm{O}_{3}-\mathrm{Al}, \mathrm{Al}_{2} \mathrm{O}_{3}$ etc. $)$.

Se realizó una descripción del método experimental de generación de NPs por ablación láser de pulsos ultracortos como así también de los mecanismos de síntesis de NPs usualmente aceptados al trabajar con este método, particularmente aplicados al caso de la fabricación de NPs de Al en agua y n-heptano. Se describieron características típicas como los límites del umbral de ablación (energía mínima necesaria para que ocurra el proceso de ablación), la profundidad de ablación (profundidad que alcanza el proceso de ablación sobre el blanco) y la cantidad de material removido durante este proceso. 
Para estudiar la influencia de parámetros como forma, tamaño y composición en los espectros de extinción de nanoestructuras esféricas con dos cubiertas, se desarrolló una extensión de la Teoría de Mie que considera las condiciones de contorno de tres interfaces para las ecuaciones de Maxwell. Bajo estas condiciones, se determinaron mapas espaciales de refuerzo de campo para NPs con un núcleo y dos cubiertas (del tipo metal-dieléctrico-metal). Se encontró que dicho refuerzo es mucho más elevado en la región intermetálica que en el exterior de la NP y que su valor absoluto depende de la combinación metal-dieléctrico-metal elegida. También se observa que las resonancias presentadas en longitudes de onda mayores presentan menor simetría debido la pérdida de predominancia de los modos dipolares. Para la posición espacial de máximo refuerzo, se calculó además su valor para un rango de longitudes de onda que abarca el UV-visible y cercano IR, observándose que los espectros presentan un máximo asociado a las resonancias plasmónicas de estas estructuras complejas. Además, con respecto al tema de plasmones de NPs que presentan cubiertas, se realizó un estudio detallado de los mismos desde el punto de vista del llamado modelo de hibridación, justificando la presencia de las distintas resonancias.

Finalmente, para estudiar los espectros de extinción de NPs de forma arbitraria, se desarrolló un programa de cálculo basado en el método de aproximación de dipolos discretos (DDA). El mismo fue validado por comparación con resultados obtenidos utilizando teoría de Mie en NPs esféricas de núcleo desnudo y núcleo-cubierta. Luego fue aplicado al caso de otras configuraciones geométricas como cubo, cubo octaedro, octaedro truncado, icosaedro y decaedro. Como demostración directa de aplicación del método DDA, el mismo fue utilizado en dos líneas de trabajo propias: determinación de la forma y distribución de tamaño de NPs de Na precipitadas en haluros alcalinos por tratamiento térmico, y descripción de las características de transmisión de cristales fotónicos sintonizables formados por arreglos de $\mathrm{NPs}$ de $\mathrm{Fe}_{3} \mathrm{O}_{4}-\mathrm{SiO}_{2}$. 


\section{Actividades en ejecución y/o futuras}

Estudio de la extinción y absorción de NPs esféricas con alto índice de refracción para describir fenómenos relacionados con el llamado "magnetismo óptico" (realce de términos dipolares magnéticos en el desarrollo en serie de la sección eficaz de scattering en teoría de Mie).

Fabricación de NPs esféricas con alto índice de refracción utilizando la ablación láser de fs y utilizando métodos químicos. Caracterización de los coloides obtenidos por distintas técnicas experimentales.

Estudio de la extinción y absorción de nanoestructuras con alto indice de refracción y de forma arbitraria, utilizando la aproximación de dipolos discretos.

Desarrollo del programas computacionales para la descripción de guías de onda plasmónicas utilizando los métodos de elementos finitos y de líneas. programas que se utilizaran para el diseño de dispositivos plasmónicos.

Fabricación por litografía de guías de onda plasmónicas y nanoestructuras metálicas de forma arbitraria para aplicaciones fotónicas.

Implementación un sensor en la configuración Kretschmann para aplicaciones de sensado bajas concentraciones. 


\section{Producido de la Tesis}

El resultados novedosos obtenidos durante el desarrollo de esta Tesis doctoral fueron publicados en revistas internacionales y fueron presentados en diversos eventos científicos nacionales e internacionales.

Trabajos publicados:

1. Luis J. Mendoza Herrera; David Muñeton Arboleda; Daniel C. Schinca; Lucia B. Scaffardi. Determination of plasma frequency, damping constant and size distribution from the complex dielectric function of noble metal nanoparticles, Journal of Applied Physics, 2014, vol. 116 n¹1. p 2331051-2331058.

2. David Muñeton Arboleda; J M J Santillan; Luis J. Mendoza Herrera; Marcela B. Fernández Van Raap; Diego Muraca; Lucia B. Scaffardi; Daniel C. Schinca. Structure, configuration, and sizing of Ni nanoparticles generated by ultrafast laser ablation in different media, SPIE.San Diego: Society of Photo-Optical Instrumentation Engineers (SPIE), 2015, vol. 9547 n¹. p 1-9.

3. David Muñeton Arboleda; J M J Santillan; Luis J. Mendoza Herrera; Marcela B. Fernández Van Raap; Pedro Mendoza Zélis; Diego Muraca; Daniel C. Schinca; Lucia B. Scaffardi. Synthesis of Ni nanoparticles by femtosecond laser ablation in liquids: Structure and sizing, Journal of Physical Chemistry C, 2015, vol. 119 n²3. p 13184-13193. 
4. Luis J. Mendoza Herrera; Ignacio Bruvera; Lucia B. Scaffardi; Daniel C. Schinca. Sizing and Eddy currents in magnetic core nanoparticles: an optical extinction approach, Physical Chemistry Chemical Physics, 2017, vol. 1 n¹. p 3076-3086.

5. Luis J. Mendoza Herrera; David Muñeton Arboleda; J M J Santillan; Marcela B. Fernández Van Raap; Lucia B. Scaffardi; Daniel C. Schinca. Nanoscale dielectric function of $\mathrm{Fe}, \mathrm{Pt}, \mathrm{Ti}, \mathrm{Ta}, \mathrm{Al}$ and $\mathrm{V}$. Application to characterization of $\mathrm{Al}$ nanoparticles synthesized by fs laser ablation, Plasmonics, 2016, vol. $1 \mathrm{n}^{\circ} 1$. p $1-12$.

6. Luis J. Mendoza Herrera; Lucia B. Scaffardi; Daniel C. Schinca. High spectral field enhancement and tunability in core-double shell metal-dielectric-metal spherical nanoparticles, RSC Advances, 2016, vol. 6 n¹12. p110471-110481.

7. David Muñeton Arboleda; J M J Santillan; Luis J. Mendoza Herrera; Diego Muraca; Lucia B. Scaffardi; Daniel C. Schinca. Size-dependent complex dielectric function of Ni, Mo, W, Pb, Zn and Na nanoparticles. Application to sizing. Journal of Physics D (Applied Physics), 2016, vol. 49 n¹. p 75302-75309.

8. Luis J. Mendoza Herrera; Ignacio Bruvera; Lucia B. Scaffardi; Daniel C. Schinca. Understanding the magnetic photonic crystals via DDA+Mie modelling: the truth of the MiLaNGA method. En redacción.

\section{Presentaciones en eventos:}

1. Luis J. Mendoza Herrera; David Muñeton Arboleda; Fabian A. Videla; Jesica M. J. Santillan; Daniel C. Schinca; Lucia B. Scaffardi. Función dieléctrica de aluminio: determinación de parámetros de bulk y comportamiento en dimensiones nanométricas. Argentina. AFA. 2013. Revista. 98 Reunión Nacional de la Asociación Física Argentina.

2. David Muñeton Arboleda; Luis J. Mendoza Herrera; Jesica M. J. Santillan; Fabian A. Videla; Daniel C. Schinca; Lucia B. Scaffardi. Resonancia plasmónica de 
nanopartículas de aluminio en estructuras núcleo-cubierta. Argentina. AFA. 2013. 98 Reunión Nacional de la Asociación Física Argentina.

3. David Muñeton Arboleda; Luis J. Mendoza Herrera; Jesica M. J. Santillan; Daniel C. Schinca; Lucia B. Scaffardi. Estudio de la resonancia plasmónica en el visble-infrarrojo cercano de nanopartículas core-shell dieléctrico-tantalio. Argentina. Córdoba. 2014. 3ra Reunión NanoCórdoba. Facultad de Ciencias Químicas de la Universidad Nacional de Córdoba (UNC).

4. Luis J. Mendoza Herrera; Fabian A. Videla; Lucia B. Scaffardi; Daniel C. Schinca. Sección eficaz de extinción y campo eléctrico dispersado por diferentes nanoestructuras simples y core-shell utilizando métodos analíticos y aproximación por dipolos discretos (DDA). Argentina. Campana. 2014. Encuentro. X Taller de Óptica y Fotofísica (TOPFOT) V Encuentro de Estudiantes de Óptica y Fotofísica (EEOF). Universidad Tecnológica Nacional, Facultad Regional Delta.

5. Luis J. Mendoza Herrera; David Muñeton Arboleda; Lucia B. Scaffardi; Daniel C. Schinca. Determinación de parámetros de Drude en metales nobles y su influencia en la función dieléctrica y en la resonancia plasmónica. Argentina. AFA. 2014. 99 Reunión de la Asociación Física Argentina. Universidad Nacional del Centro de la Provincia de Buenos Aires.

6. David Muñeton Arboleda; Jesica M. J. Santillan; Luis J. Mendoza Herrera; Daniel C. Schinca; Lucia B. Scaffardi. Determinación de parámetros de Drude para níquel y banda plasmónica sintonizable de Nps core-shell dieléctrico-níquel. Argentina. AFA. 2014. 99 Reunión de la Asociación Física Argentina. Universidad Nacional del Centro de la Provincia de Buenos Aires.

7. Luis J. Mendoza Herrera; Daniel C. Schinca; Lucia B. Scaffardi. Fabricación y Caracterización de nanopartículas. Jornada de Tesistas 2014.

8. David Muñeton Arboleda; Jesica M. J. Santillan; Luis J. Mendoza Herrera; Marcela B. Fernández Van Raap; Diego Muraca; Daniel C. Schinca; Lucia B. 
Scaffardi. Structure, configuration and sizing of Ni nanoparticles generated by ultrafast laser ablationin different media. Estados Unidos de América. San diego. 2015. Congreso. Optics + Photonics 2015.

9. Luis J. Mendoza Herrera; Lucia B. Scaffardi; Daniel C. Schinca. Diseño de sensor plasmónico para medida de cambios en la temperatura del agua.. Argentina. Corrientes. 2015. Taller. EEOF-TOPFOT-2015. Universidad Nacional del Nordeste.

10. Jesica M. J. Santillan; David Muñeton Arboleda; Luis J. Mendoza Herrera; Lucia B. Scaffardi; Daniel C. Schinca. Comportamiento electrónico de metales de transición en la nanoescala. Argentina. San Luis. 2015. Revista. 100 Reunión Nacional de la Asociación Física Argentina. Centro Educativo 27 Gdor. Santiago Besso.

11. Luis J. Mendoza Herrera; David Muñeton Arboleda; Lucia B. Scaffardi; Daniel C. Schinca. Constante de amortiguamiento y frecuencia de plasma para Pt, Ti y Ta. Argentina. Rosario. 2015. Encuentro. XV Encuentro: Superficies y Materiales Nanoestructurados NANO 2015. Universidad Nacional.

12. Luis J. Mendoza Herrera; Lucia B. Scaffardi; Daniel C. Schinca. Función dieléctrica efectiva en nanopartículas núcleo-doble cubierta. Aplicación a nanopartículas Aire-Al-Al2O3. Argentina. Rosario. 2015. Encuentro. XV Encuentro: Superficies y Materiales Nanoestructurados NANO 2015. Universidad Nacional de Rosario.

13. Luis J. Mendoza Herrera; Ignacio Bruvera; Daniel C. Schinca; Lucia B. Scaffardi. Caracterización de nanoparticulas de $\mathrm{Fe}_{3} \mathrm{O}_{4}$ - $\mathrm{Au}$ por espectroscopia de extinción y estudio del refuerzo de campo en estructuras metal-dieléctrico-metal. Chile. Púcon. 2016. Otro. RIAO 2016. Centro de Óptica y Fotónica de la Universidad de Concepción (CEFOP-UDEC). 
14. Luis J. Mendoza Herrera; Ignacio Bruvera; Daniel C. Schinca; Lucia B. Scaffardi. Caracterización de nanopartículas de Fe3O4 y estudio de nanopartículas Fe3O4-SiO2-Au. Argentina. Buenos Aires. 2016. Taller. EOF-TOPFOT 2016. Universidad de Buenos Aires.

15. Luis J. Mendoza Herrera; David Muñeton Arboleda; Jesica M. J. Santillan; Daniel C. Schinca; Lucia B. Scaffardi. Propiedades ópticas y distribución de tamaño de nanopartículas de Al generadas por ablación láser utilizando espectroscopía de extinción.. Argentina. Buenos Aires. 2016. Taller. EOF-TOPFOT 2016. Universidad de Buenos Aires.

16. Luis J. Mendoza Herrera; Daniel C. Schinca; Lucia B. Scaffardi. Sintonía de resonancias plasmónicas y refuerzo de campo en estructuras nanométricas Metal-Dieléctrico-Au-oro.. Argentina. Buenos Aires. 2016. Encuentro. Escuela en Nanociencia y Nanotecnología, Nano2016 y XVI Encuentro de Superficies y Materiales Nanoestructurados, Nano 2016. Universidad de Buenos Aires.

17. Luis J. Mendoza Herrera; Lucia B. Scaffardi; Daniel C. Schinca. Caracterización de nanopatículas de Na utilizando DDA. Argentina. AFA. 2016. 101 Reunión de la Asociación Física Argentina. Universidad Nacional de Tucumán. Tucumán.

18. Luis J. Mendoza Herrera; Ignacio Bruvera; Daniel C. Schinca; Lucia B. Scaffardi. Caracterización de nanoparticulas de Fe3O4-Au por espectroscopia de extinción y estudio del refuerzo de campo en estructuras metal-dieléctrico-metal. 2016. IX Reunión Iberoamericana de Óptica y XII Reunión Iberoamericana de Óptica, Láseres y Aplicaciones (RIAO/OPTILAS). Centro de Óptica y Fotónica de la Universidad de Concepción. Púcon. Chile.

19. Luis J. Mendoza Herrera; Ignacio Bruvera; Daniel C. Schinca; Lucia B. Scaffardi. Importancia de las corrientes de Eddy en la extinción de nanopartículas: Caracterización de tamaños. NacoCórdoba 2017.Universidad Nacional de Córdoba. Villa Carlos Paz. Argentina. 


\section{Apéndice A Gráficas función dieléctrica}

La vida es linda, lo malo es que muchos confunden lindo con fácil.

Mafalda.

\section{A.1. Otras funciones dieléctricas utilizadas}

Para el modelado de los espectros de extinción, absorción, scattering y refuerzos de campo, además de las funciones dieléctricas de los metales, se utilizan las funciones dieléctricas de diversos materiales $\left(\mathrm{Al}_{2} \mathrm{O}_{3}, \mathrm{SiO}_{2}, \mathrm{TiO}_{2}\right.$ y $\left.\mathrm{H}_{2} \mathrm{O}\right)$. Estas funciones dieléctricas se describen a continuación:

Para el $\mathrm{Al}_{2} \mathrm{O}_{3}$, descrito en la referencia 160

$$
n^{2}-1=\frac{1,4313493 \lambda^{2}}{\lambda^{2}-0,0726631^{2}}+\frac{0,65054713 \lambda^{2}}{\lambda^{2}-0,1193242^{2}}+\frac{5,3414021 \lambda^{2}}{\lambda^{2}-18,028251^{2}},
$$

para el $\mathrm{SiO}_{2}$, descrito en la referencia [161]

$$
n^{2}-1=\frac{0,6961663 \lambda^{2}}{\lambda^{2}-0,0684043^{2}}+\frac{0,4079426 \lambda^{2}}{\lambda^{2}-0,1162414^{2}}+\frac{0,8974794 \lambda^{2}}{\lambda^{2}-9,896161^{2}},
$$

para el $\mathrm{TiO}_{2}$, descrito en la referencia 162

$$
n^{2}=5,913+\frac{0,2441}{\lambda^{2}-0,0803}
$$

y para el $\mathrm{H}_{2} \mathrm{O}$, descrito en la referencia $\mathbf{1 6 3}$ 


$$
\begin{aligned}
n^{2}-1 & =\frac{5,684027565 \times 10^{-1} \lambda^{2}}{\lambda^{2}-5,101829712 \times 10^{-3}}+\frac{1,726177391 \times 10^{-1} \lambda^{2}}{\lambda^{2}-1,821153936 \times 10^{-2}} \\
& +\frac{2,086189578 \times 10^{-2} \lambda^{2}}{\lambda^{2}-2,620722293 \times 10^{-2}}+\frac{1,130748688 \times 10^{-1} \lambda^{2}}{\lambda^{2}-1,069792721 \times 10}
\end{aligned}
$$

\section{A.2. Gráficas de la función dieléctrica}

En el Capítulo 2, se describe un método para determinar los parámetros característicos del modelo de Drude $\left(\omega_{p}\right.$ y $\left.\gamma\right)$ a partir del conjunto de ecuaciones 2.10 2.11. Los resultados de las gráficas de los valores experimentales de $\omega \varepsilon^{\prime \prime}(\omega)$ y $\omega^{2}\left[\left(\varepsilon^{\prime \prime}(\omega)\right)^{2}+\left(1-\varepsilon^{\prime}(\omega)\right)^{2}\right]$ en función de $1-\varepsilon^{\prime}(\omega)$ para $\mathrm{Ag}, \mathrm{Cu}$ y $\mathrm{Al}$ se ilustran en las Figuras A.1 A.3. En círculos rojos $\omega \varepsilon^{\prime \prime}(\omega)$ en función de $1-\varepsilon^{\prime}(\omega)$ y en cuadrados grises $\omega^{2}\left[\left(\varepsilon^{\prime \prime}(\omega)\right)^{2}+\left(1-\varepsilon^{\prime}(\omega)\right)^{2}\right]$ en función de $1-\varepsilon^{\prime}(\omega)$. Adicionalmente se muestran los ajustes de estos valores experimentales en la región donde la contribución de los electrones ligados presentan poca influencia.

Utilizando los parámetros de Drude determinados para los metales Ag, $\mathrm{Cu}$ y Al, en las Figuras A.4 A.6 se compara el ajuste de los valores experimentales con la función dieléctrica calculada con el modelo de Drude utilizando valores de los parámetros reportados por otros autores junto con nuestros resultados. Se observa claramente una mejor concordancia de nuestros valores con los datos experimentales.

En las Figuras A.7 A.9 se muestran las partes real e imaginaria de la función dieléctrica de los metales $\mathrm{Ag}, \mathrm{Cu}$ y $\mathrm{Al}$, corregidas por tamaño, para radios entre $2 \mathrm{~nm}$ y $50 \mathrm{~nm}$. 

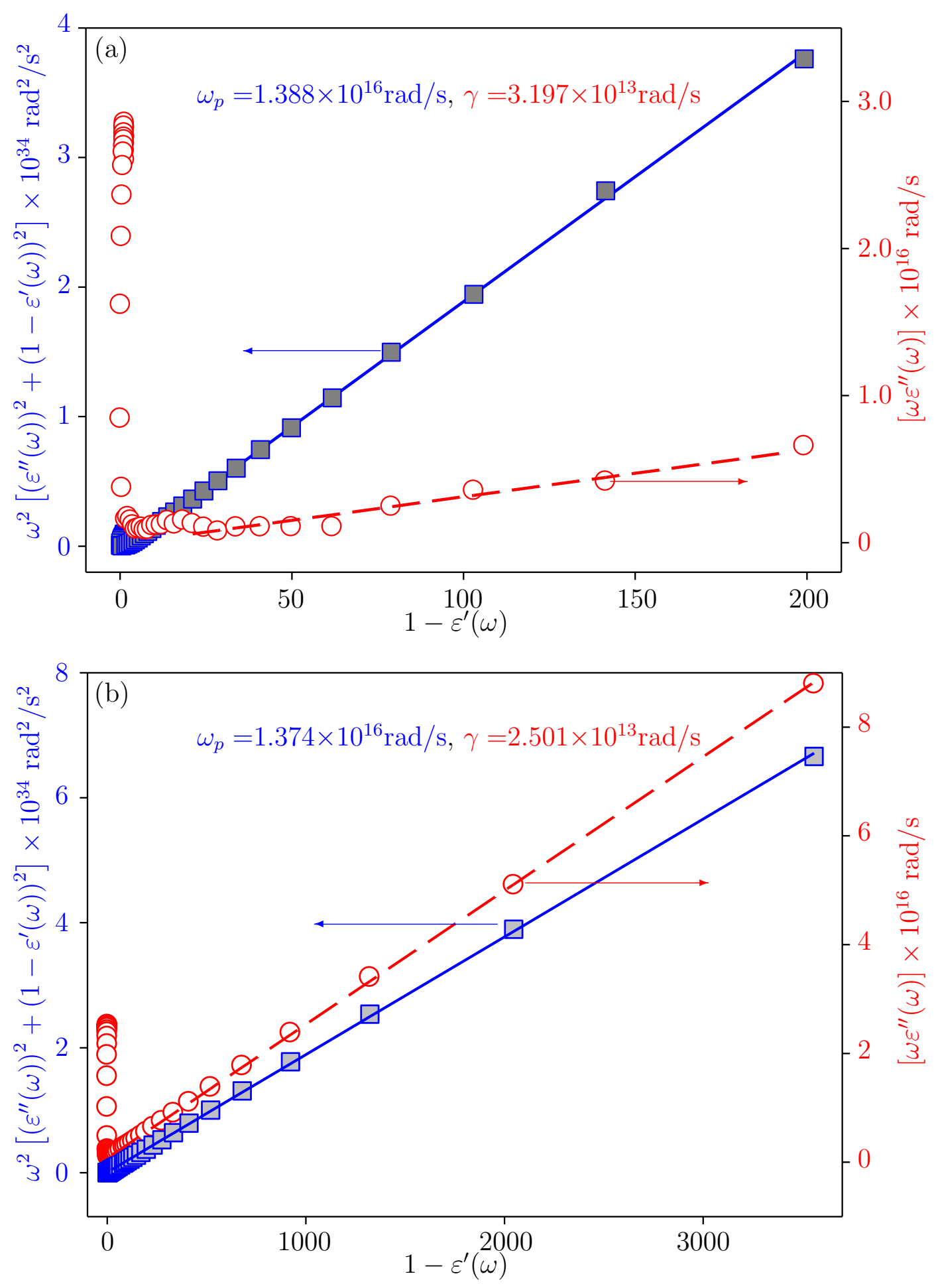

Figura A.1: Gráficas de las ecuaciones 2.10 y 2.11 para Ag (a) P. B. Johnson-1972 y (b) S. Babar-2015. Ajuste de los datos por regresión lineal para el rango donde la función dieléctrica es descrita por el modelo de Drude. Las pendientes obtenidas por la regresión lineal representan $\omega_{p}^{2}$ (línea llena azul) y $\gamma_{\text {libres }}$ (línea segmentada roja). 

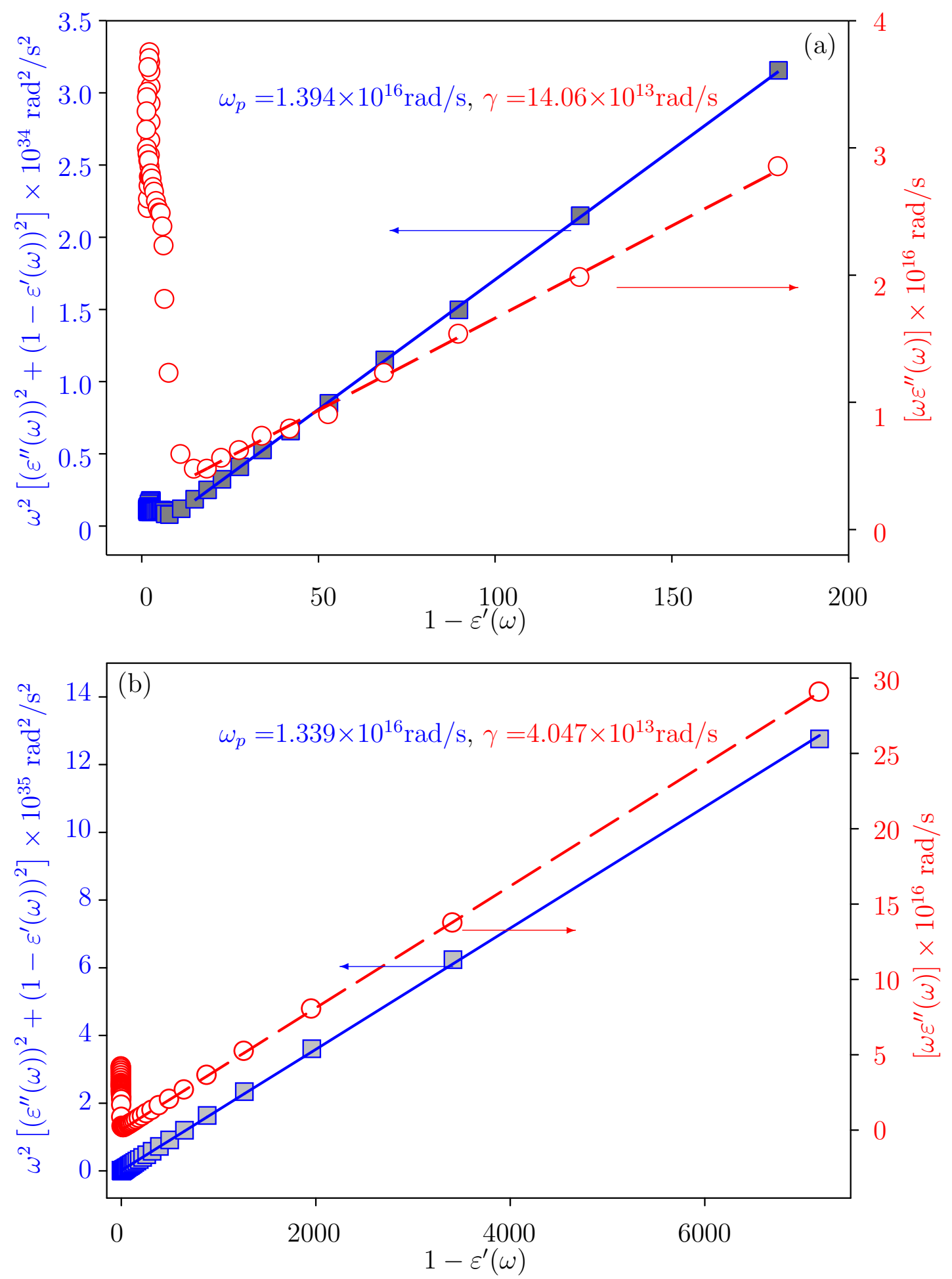

Figura A.2: Gráficas de las ecuaciones 2.10 y 2.11 para $\mathrm{Cu}$ (a) (a) P. B. Johnson-1972 y (b) S. Babar-2015. Ajuste de los datos por regresión lineal para el rango donde la función dieléctrica es descrita por el modelo de Drude. Las pendientes obtenidas por la regresión lineal representan $\omega_{p}^{2}$ (línea llena azul) y $\gamma_{\text {libres }}$ (línea segmentada roja). 

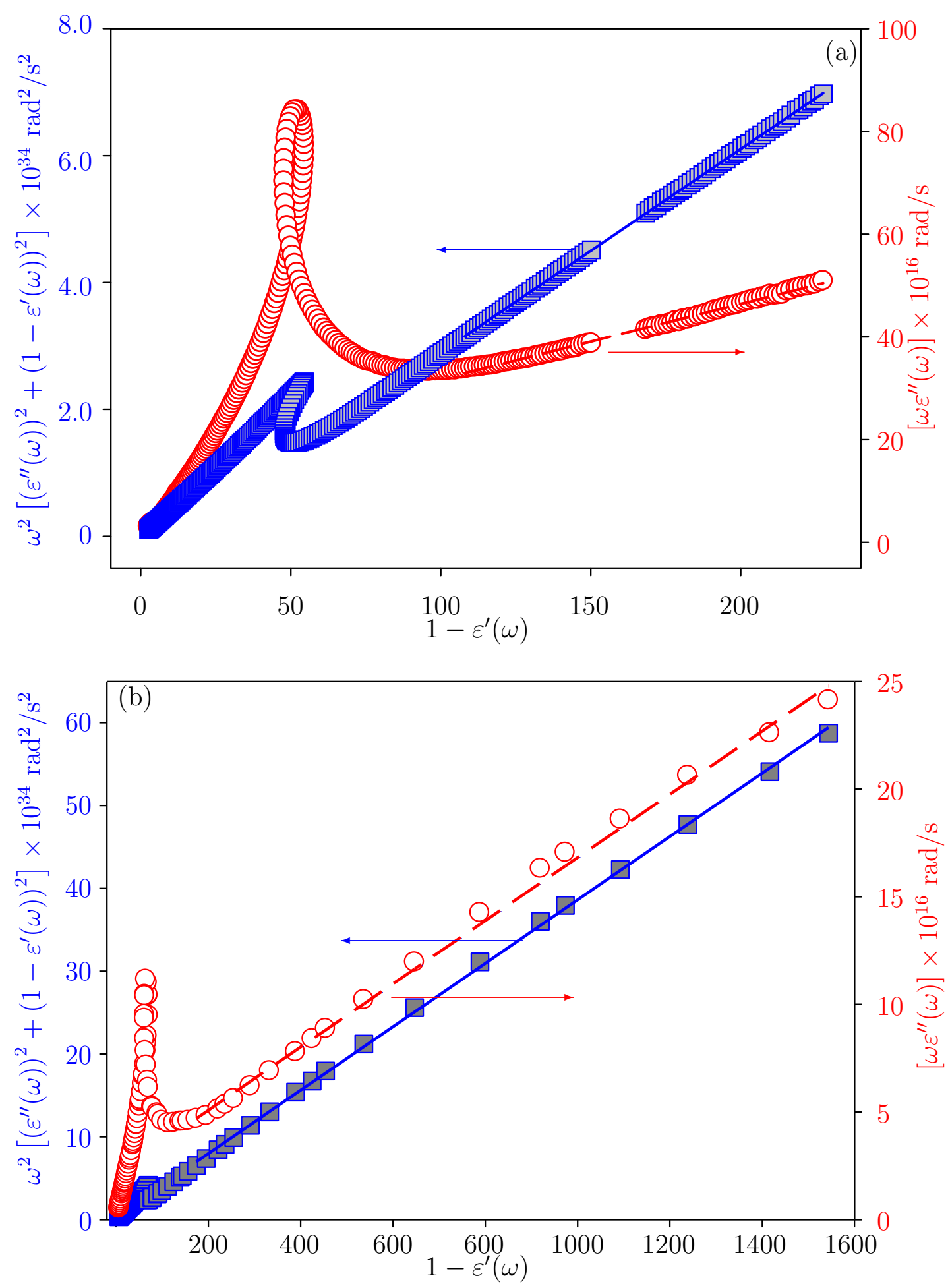

Figura A.3: Gráficas de las ecuaciones 2.10 y 2.11 para Al (a) K. M. McPeak-2015 y (b) A. D. Rakic-1998. Ajuste de los datos por regresión lineal para el rango donde la función dieléctrica es descrita por el modelo de Drude. Las pendientes obtenidas por la regresión lineal representan $\omega_{p}^{2}$ (línea llena azul) y $\gamma_{\text {libres }}$ (línea segmentada roja). 

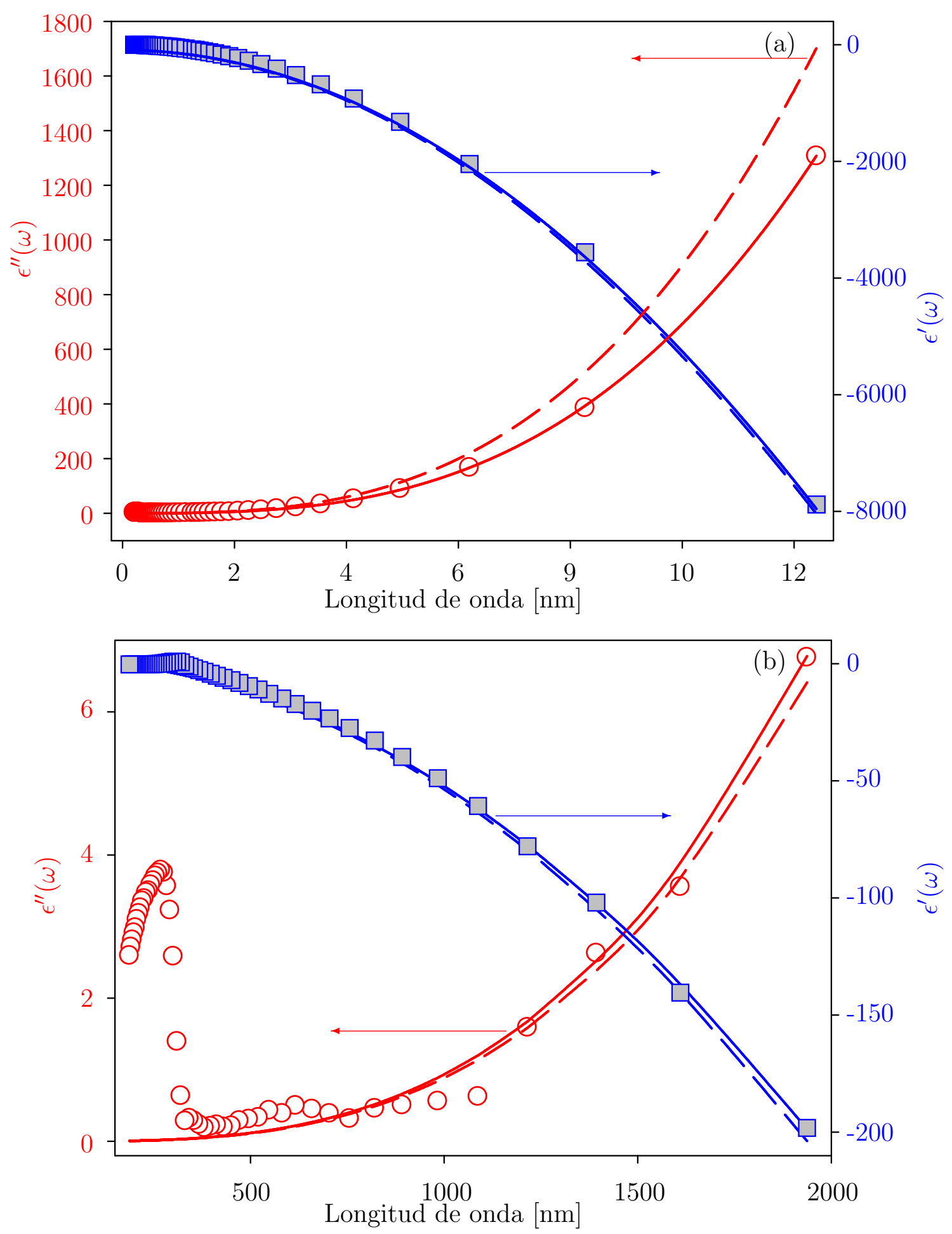

Figura A.4: Partes real e imaginaria de la contribución de los electrones libres a la función dieléctrica (a) Ag S. Babar-2015 y (b) Ag P. B. Johnson-1972, utilizando los valores de $\omega_{p}$ y $\gamma_{\text {libres }}$ obtenidos de las ecuaciones 2.10 y 2.11 en línea continua y en línea segmentada para los valores de $\omega_{p}$ y $\gamma_{\text {libres }}$ dados en P. B. Johnson-1972. Los cuadrados azules y círculos rojos representan las partes real e imaginaria de la función dieléctrica experimental. 

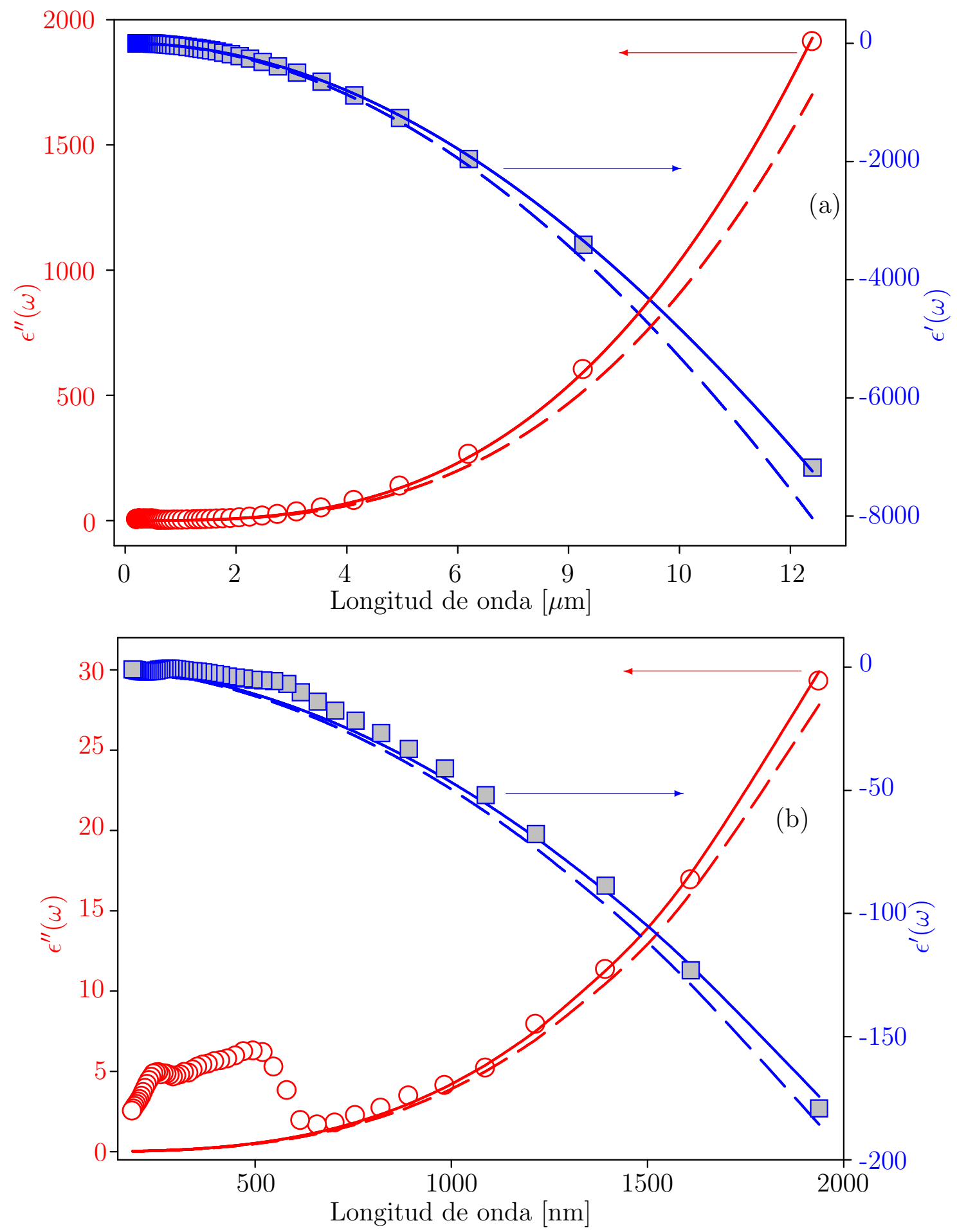

Figura A.5: Partes real e imaginaria de la contribución de los electrones libres a la función dieléctrica (a) Cu S. Babar-2015 y (b) Cu P. B. Johnson-1972, utilizando los valores de $\omega_{p}$ y $\gamma_{\text {libres }}$ obtenidos de las ecuaciones 2.10 y 2.11 en línea continua, utilizando los valores dados en P. B. Johnson-1972 para el $\mathrm{Cu}$ en segmentos largos y los valores dados en A. D. Rakic-1998 y M.A. Ordal-1988 en segmentos largos y cortos respectivamente para el $\mathrm{Cu}$. Los cuadrados azules y círculos rojos representan las partes real e imaginaria de la función dieléctrica experimental. 

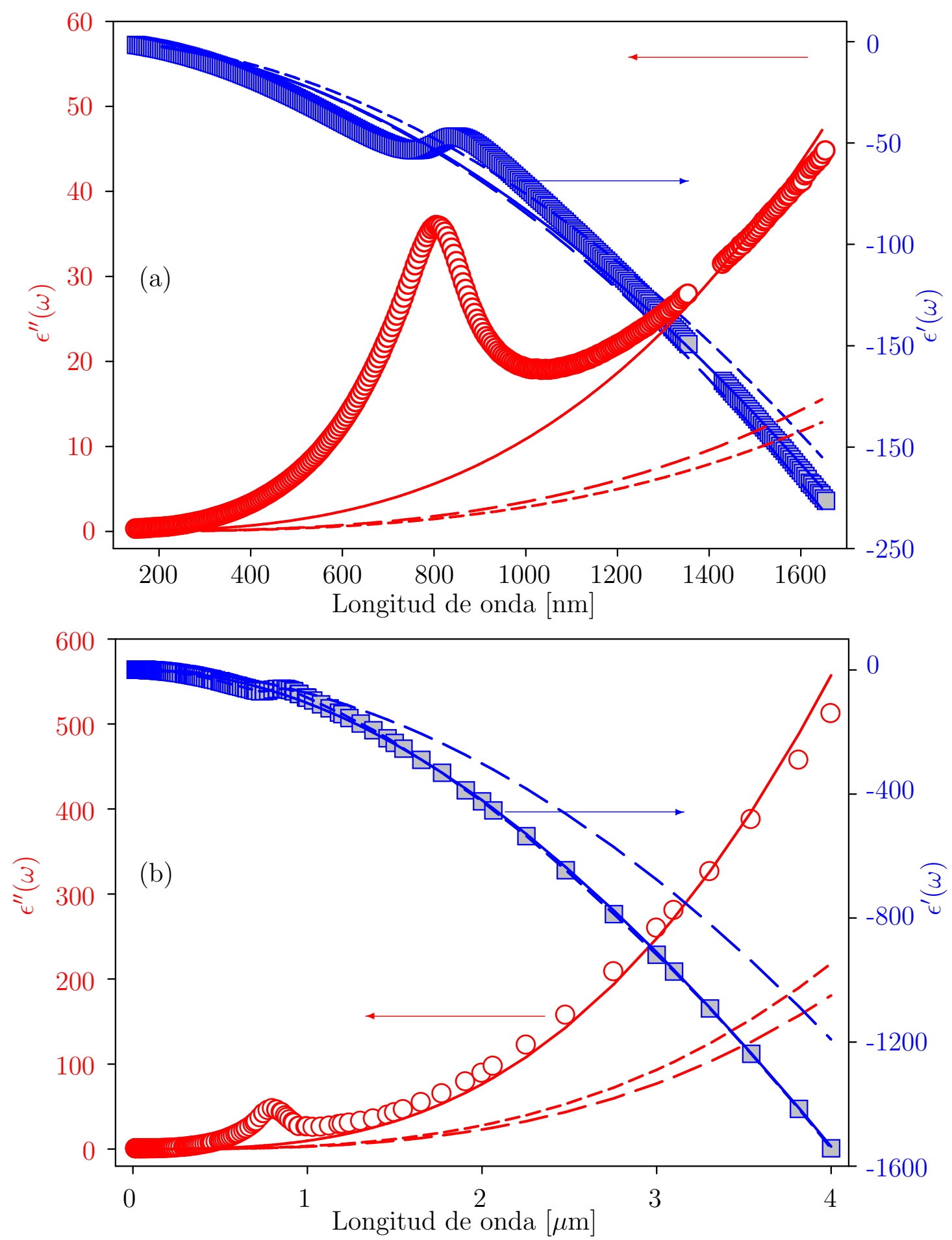

Figura A.6: Partes real e imaginaria de la contribución de los electrones libres a la función dieléctrica (a) Al K. M. McPeak-2015 y (b) Al A. D. Rakic-1998, utilizando los valores de $\omega_{p}$ y $\gamma_{\text {libres }}$ obtenidos de las ecuaciones 2.10 y 2.11 en línea continua, utilizando los valores dados en P. B. Johnson-1972 para el Al en segmentos largos y los valores dados en A. D. Rakic-1998 y M.A. Ordal-1988 en segmentos largos y cortos respectivamente para el Al. Los cuadrados azules y círculos rojos representan las partes real e imaginaria de la función dieléctrica experimental. 

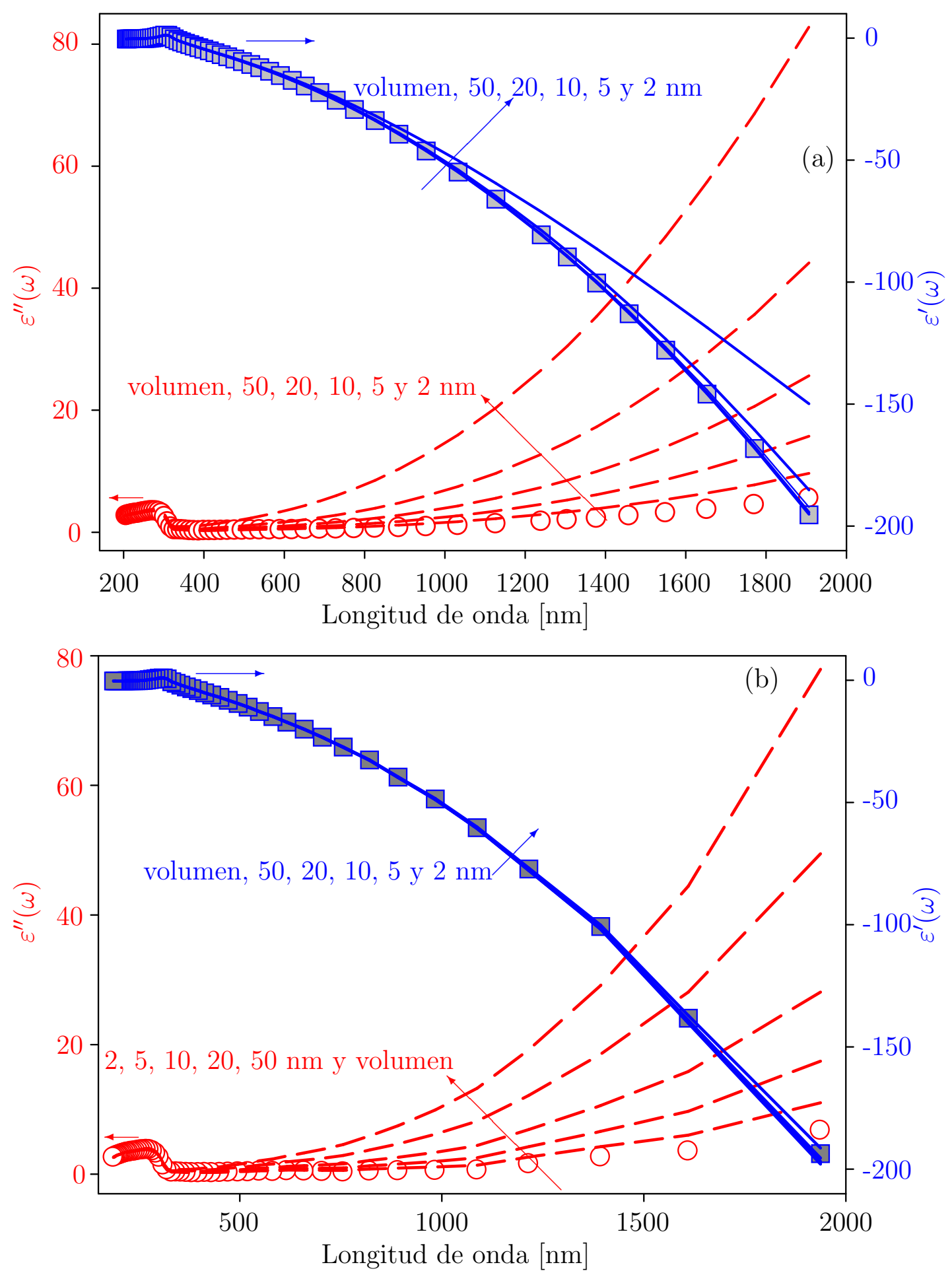

Figura A.7: Partes real (línea continua) e imaginaria (línea segmentada) de la contribución de los electrones libres a la función dieléctrica (a) Ag S. Babar-2015 y (b) Ag P. B. Johnson-1972, para diferentes radios de nanopartículas. Los cuadrados azules y círculos rojos representan las partes real e imaginaria de la función dieléctrica experimental. 

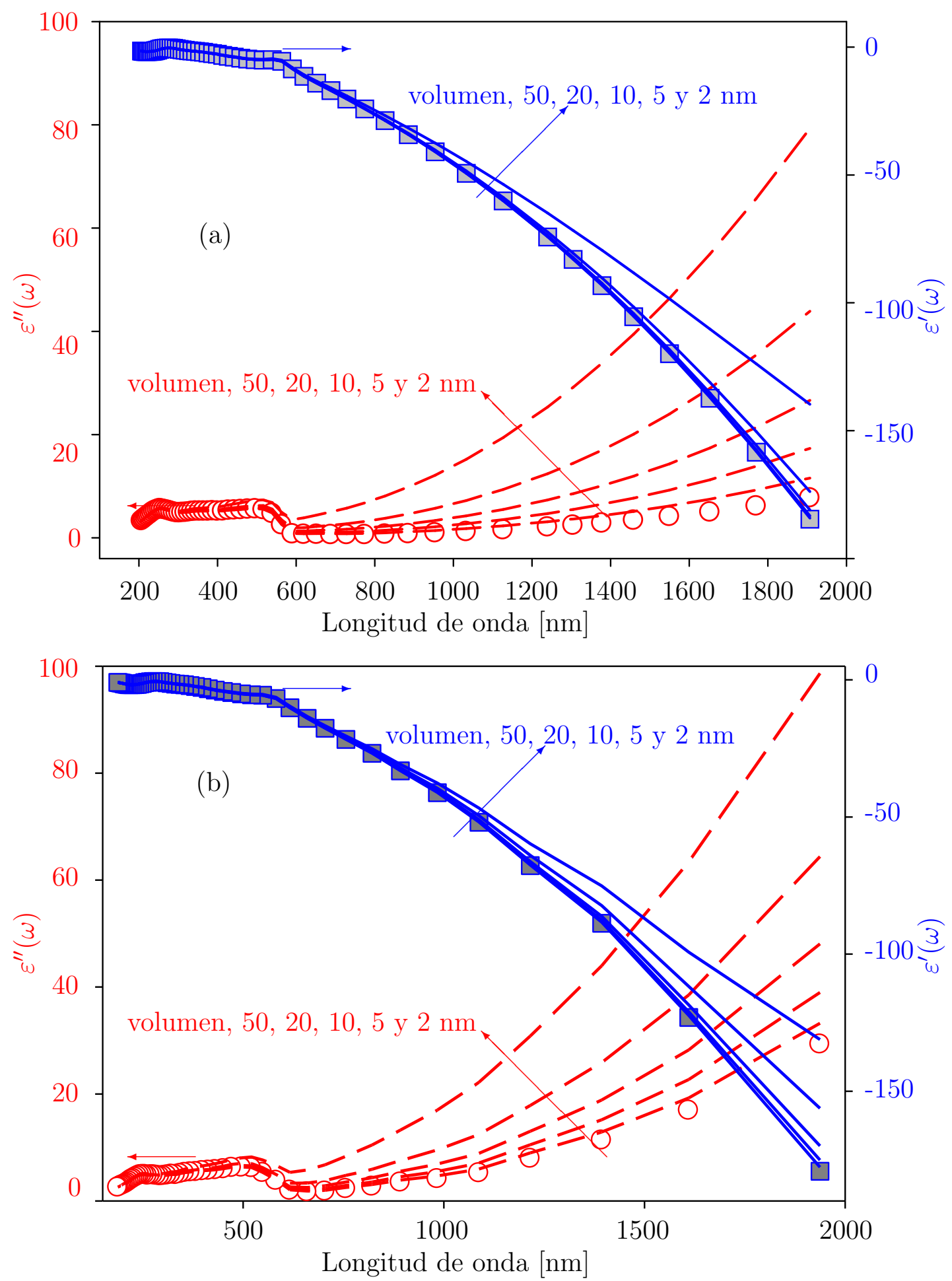

Figura A.8: Partes real (línea continua) e imaginaria (línea segmentada) de la contribución de los electrones libres a la función dieléctrica (a) $\mathrm{Cu} \mathrm{S}$. Babar-2015 y (b) Cu P. B. Johnson-1972, para diferentes radios de nanopartículas. Los cuadrados azules y círculos rojos representan las partes real e imaginaria de la función dieléctrica experimental. 

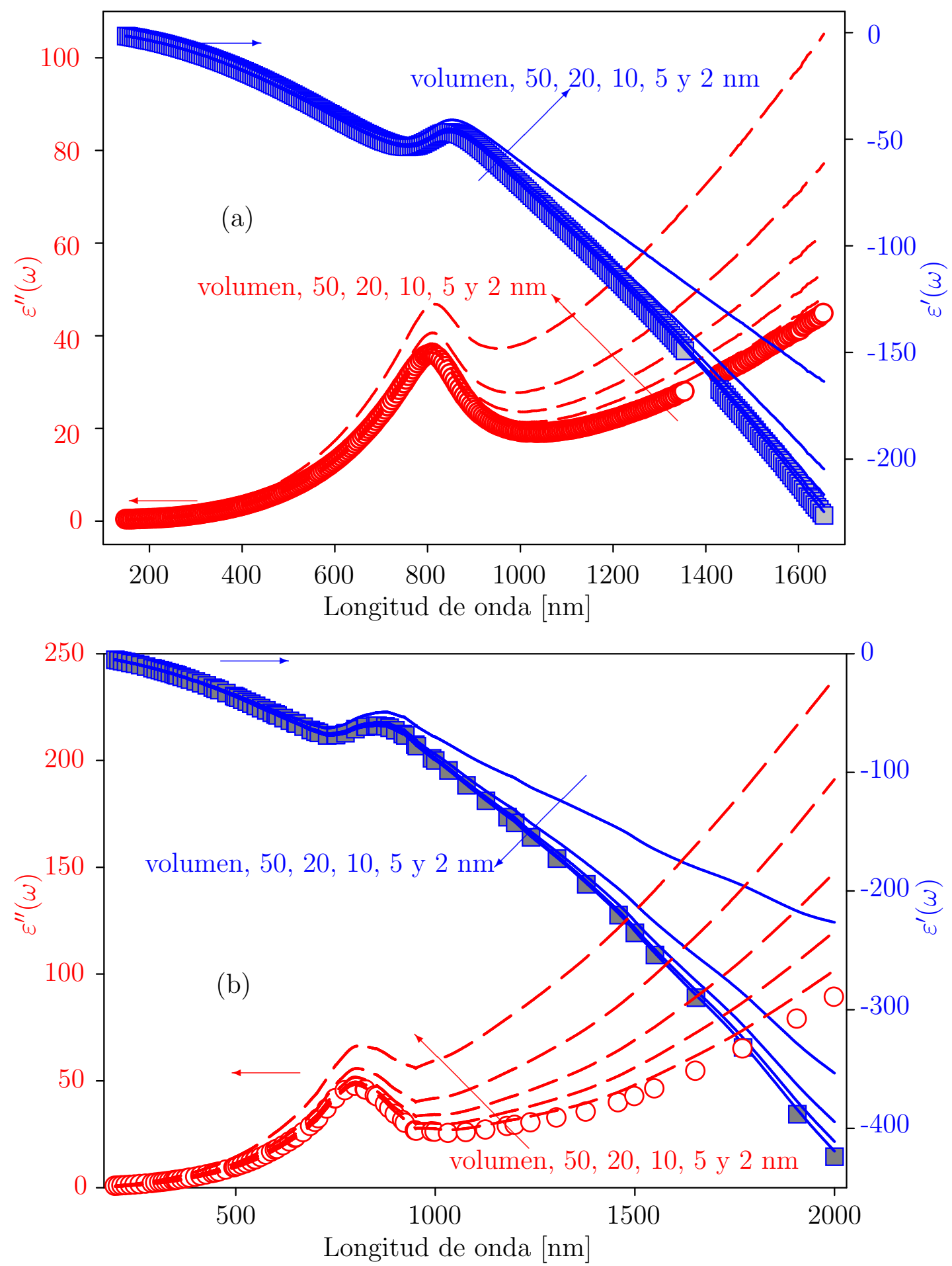

Figura A.9: Partes real (línea continua) e imaginaria (línea segmentada) de la contribución de los electrones libres a la función dieléctrica (a) Al K. M. McPeak-2015 y (b) Al A. D. Rakic-1998, para diferentes radios de nanopartículas. Los cuadrados azules y círculos rojos representan las partes real e imaginaria de la función dieléctrica experimental. 


\section{Apéndice $B$ Extinción de nanopartículas esféricas}

La verdad puede eclipsarse pero no extinguirse

Tito Livio.

En este Apéndice se desarrolla la teoría de Mie para NPs de la forma núcleo doble cubierta, ilustrando como se reduce la misma para NPs núcleo-cubierta y desnudas. También se muestra está teoría en el caso de NPs muy pequeñas (Aproximación dipolar).

En el caso de NPs magnéticas se desarrolla deduce la polarizabilidad debida a las corrientes de Eddy para NPs núcleo doble cubierta, extendida a partir de los desarrollos en partículas esféricas desnudas realizados por Landau.

\section{B.1. Ecuación de Ondas}

Para el cálculo de los campos eléctricos y magnéticos dentro y fuera de una NP esférica, se debe solucionar la ecuación vectorial de ondas en coordenadas esféricas [4, 11-13].

$$
\nabla^{2} \mathbf{E}+k^{2} \mathbf{E}=0, \quad \nabla^{2} \mathbf{H}+k^{2} \mathbf{H}=0
$$

donde $k=\sqrt{\varepsilon \mu} \omega$ es el número de onda, $\mu$ es la permeabilidad magnética, $\varepsilon$ es la función dieléctrica y $\omega$ es la frecuencia. Un método para obtener la solución de la ecuación vectorial de ondas consiste en solucionar la ecuación escalar de ondas en coordenadas esféricas y a partir de las soluciones de la ecuación escalar construir las 
soluciones de la ecuación vectorial $4, \mathbf{1 1}-\mathbf{1 3}$.

$$
\frac{1}{r^{2}} \frac{\partial}{\partial r}\left(r^{2} \frac{\partial \psi}{\partial r}\right)+\frac{1}{r^{2} \operatorname{sen} \theta} \frac{\partial}{\partial \theta}\left(\operatorname{sen} \theta \frac{\partial \psi}{\partial \theta}\right)+\frac{1}{r^{2} \operatorname{sen} \theta} \frac{\partial^{2} \psi}{\partial \phi^{2}}+k^{2} \psi=0
$$

utilizando el método de separación de variables, las soluciones de la ecuación escalar de ondas en coordenadas esféricas se describen como [13]:

$$
\begin{aligned}
& \psi_{e m n}=\cos m \psi P_{n}^{m}(\cos \theta) z_{n}(k r) \\
& \psi_{o m n}=\operatorname{sen} m \psi P_{n}^{m}(\cos \theta) z_{n}(k r)
\end{aligned}
$$

donde $P_{n}^{m}(\cos \theta)$, son los polinomios asociados de Legendre y $z_{n}(k r)$ son las funciones esféricas de Bessel: $j_{n}(k r), y_{n}(k r), h_{n}^{(1)}=j_{n}(k r)+i y_{n}(k r)$ ó $h_{n}^{(2)}=j_{n}(k r)-$ $i y_{n}(k r)$, las cuales son definidas en términos de las funciones de Bessel como [164, 165]:

$$
\begin{aligned}
j_{n}(k r) & =\sqrt{\frac{\pi}{2 k r}} J_{n+1 / 2}(k r) \\
y_{n}(k r) & =\sqrt{\frac{\pi}{2 k r}} Y_{n+1 / 2}(k r)
\end{aligned}
$$

donde $J_{n}(k r)$ y $Y_{n}(k r)$, son las funciones de Bessel de primera y segunda clase.

En las Figuras B.1.a y B.1.b, se representan las funciones esféricas de Bessel $j_{n}(k r)$ y $y_{n}(k r)$, en las cuales se observa que para valores pequeños de $k r$, la función $y_{n}(k r)$ diverge y por tanto en las soluciones para el campo en el interior de la esfera no se deben considerar. 

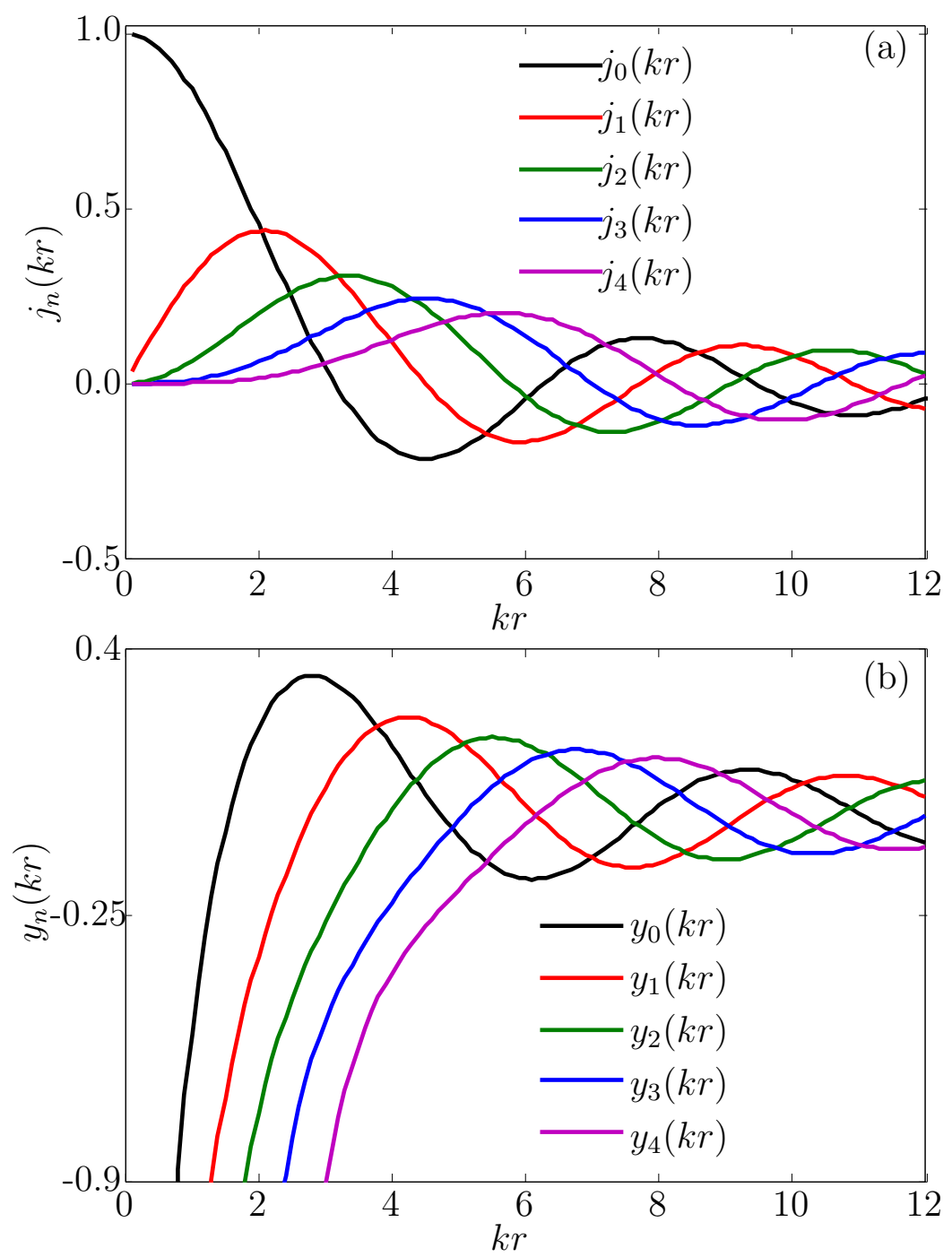

Figura B.1: Gráfico de las funciones esféricas de Bessel (a) $j_{n}(k r)$ y (b) $y_{n}(k r)$, en función de $k r$.

Las soluciones a la ecuación vectorial de ondas que denotaremos por $\mathbf{M}_{e m n}, \mathbf{M}_{o m n}$, $\mathbf{N}_{\text {emn }}$ y $\mathbf{N}_{\text {omn }}$ se construyen a partir de las soluciones de la ecuación escalar como 13, 166, 167]:

$$
\begin{aligned}
\mathbf{M}_{e m n}=\nabla \times\left(\mathbf{r} \psi_{e m n}\right), & \mathbf{M}_{o m n}=\nabla \times\left(\mathbf{r} \psi_{o m n}\right) \\
\mathbf{N}_{e m n}=\frac{\nabla \times\left(\mathbf{r} \psi_{e m n}\right)}{k}, & \mathbf{N}_{o m n}=\frac{\nabla \times\left(\mathbf{r} \psi_{o m n}\right)}{k}
\end{aligned}
$$




\section{B.2. Campos en nanopartículas esféricas con dos cubiertas}

El campo incidente y los campos en cada una de las regiones que se consideren se construyen como combinaciones lineales de cada una de las soluciones de la ecuación vectorial de ondas. La onda incidente se puede considerar como una onda armónica cuyo campo eléctrico es de la forma:

$$
\mathbf{E}_{i}=E_{0} e^{i k r \cos \theta} \hat{a}_{x}
$$

este campo se debe expresar como una combinación de las soluciones a la ecuación de vectorial de ondas en coordenadas esféricas. Bohren [13 muestra que las únicas soluciones que no se anulan son las correspondientes a $m=1$, produciendo una expresión para el campo eléctrico incidente de la forma:

$$
\mathbf{E}_{i}=E_{0} \sum_{n=1}^{\infty} i^{n} \frac{2 n+1}{n(n+1)}\left(\mathbf{M}_{o 1 n}^{(1)}-i \mathbf{N}_{e 1 n}^{(1)}\right)
$$

donde el superíndice ${ }^{(1)}$ indica que la función de Bessel que se utiliza es $j_{n}(k r)$, a causa de que el campo incidente se distribuye en todo el espacio y la función $y_{n}(k r)$, haría que el campo divergiera para valores pequeños de $k r$, en este caso el correspondiente campo magnético asociado al campo eléctrico incidente es:

$$
\mathbf{H}_{i}=-\frac{k}{\omega \mu_{i}} E_{0} \sum_{n=1}^{\infty} i^{n} \frac{2 n+1}{n(n+1)}\left(\mathbf{M}_{e 1 n}^{(1)}+i \mathbf{N}_{o 1 n}^{(1)}\right)
$$

Si consideramos una NP formada por un núcleo de radio $R_{1}$, y dos cubiertas de radios $R_{2}$ y $R_{3}$, con índices de refracción $N_{1}, N_{2}$ y $N_{3}$ respectivamente y la $\mathrm{NP}$ se encuentra en un medio de índice de refracción $N$ Figura B.2, sobre la que incide la onda. 


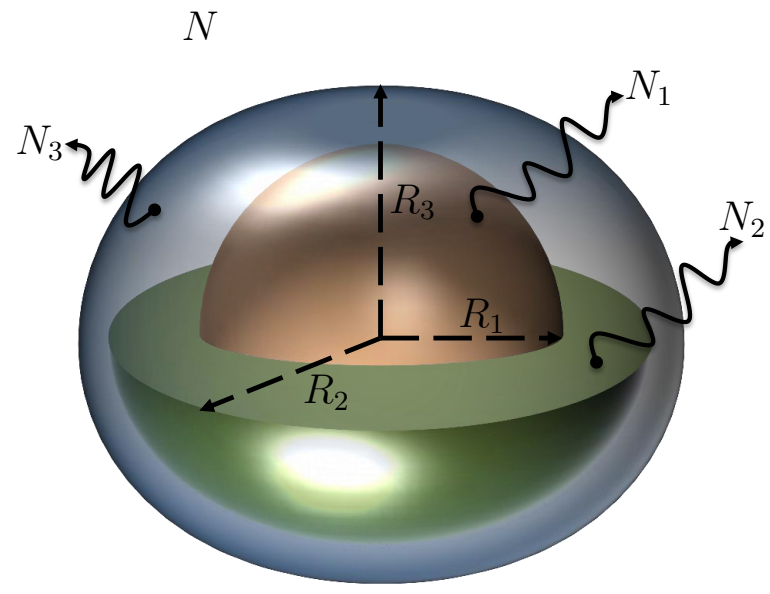

Figura B.2: Configuración geométrica para una partícula esférica con núcleo de radio $R_{1}$ y cubiertas de radios $R_{2}$ y $R_{3}$, con índices de refracción $N_{1}, N_{2}$ y $N_{3}$ respectivamente inmersa en un medio de índice de refracción $N$.

Las expresiones para los campos eléctricos en cada una de las regiones y en el exterior de la NP (campo scattereado) son:

$$
\begin{aligned}
& \mathbf{E}_{1}=\sum_{n=1}^{\infty} E_{n}\left(c_{n} \mathbf{M}_{o 1 n}^{(1)}-i d_{n} \mathbf{N}_{e 1 n}^{(1)}\right) \\
& \mathbf{E}_{2}=\sum_{n=1}^{\infty} E_{n}\left(f_{n} \mathbf{M}_{o 1 n}^{(1)}-i g_{n} \mathbf{N}_{e 1 n}^{(1)}+v_{n} \mathbf{M}_{o 1 n}^{(2)}-i w_{n} \mathbf{N}_{e 1 n}^{(2)}\right) \\
& \mathbf{E}_{3}=\sum_{n=1}^{\infty} E_{n}\left(l_{n} \mathbf{M}_{o 1 n}^{(1)}-i o_{n} \mathbf{N}_{e 1 n}^{(1)}+p_{n} \mathbf{M}_{o 1 n}^{(2)}-i q_{n} \mathbf{N}_{e 1 n}^{(2)}\right) \\
& \mathbf{E}_{s}=\sum_{n=1}^{\infty} E_{n}\left(i a_{n} \mathbf{N}_{e 1 n}^{(3)}-b_{n} \mathbf{M}_{o 1 n}^{(3)}\right)
\end{aligned}
$$

donde los superíndices ${ }^{(2)}$ y ${ }^{(3)}$ indican que las funciones de Bessel que se utilizan son $y_{n}(k r)$ y $h_{n}^{(1)}(k r)$, respectivamente y $E_{n}=i^{n} \frac{2 n+1}{n(n+1)}$. Los campos magnéticos asociados a cada uno de estos campos eléctricos son: 


$$
\begin{aligned}
& \mathbf{H}_{1}=-\frac{k_{1}}{\omega \mu_{1}} \sum_{n=1}^{\infty} E_{n}\left(d_{n} \mathbf{M}_{e 1 n}^{(1)}+i c_{n} \mathbf{N}_{o 1 n}^{(1)}\right) \\
& \mathbf{H}_{2}=-\frac{k_{2}}{\omega \mu_{2}} \sum_{n=1}^{\infty} E_{n}\left(g_{n} \mathbf{M}_{e 1 n}^{(1)}+i f_{n} \mathbf{N}_{o 1 n}^{(1)}+w_{n} \mathbf{M}_{e 1 n}^{(2)}+i v_{n} \mathbf{N}_{o 1 n}^{(2)}\right) \\
& \mathbf{H}_{3}=-\frac{k_{3}}{\omega \mu_{3}} \sum_{n=1}^{\infty} E_{n}\left(o_{n} \mathbf{M}_{e 1 n}^{(1)}+i l_{n} \mathbf{N}_{o 1 n}^{(1)}+q_{n} \mathbf{M}_{e 1 n}^{(2)}+i p_{n} \mathbf{N}_{o 1 n}^{(2)}\right) \\
& \mathbf{H}_{s}=\frac{k}{\omega \mu} \sum_{n=1}^{\infty} E_{n}\left(i b_{n} \mathbf{N}_{o 1 n}^{(3)}+a_{n} \mathbf{M}_{e 1 n}^{(3)}\right)
\end{aligned}
$$

donde,

$$
\begin{aligned}
\mathbf{M}_{o 1 n}= & \cos \phi \pi_{n}(\cos \theta) z_{n}(\rho) \hat{a}_{\theta}-\operatorname{sen} \phi \tau_{n}(\cos \theta) z_{n}(\rho) \hat{a}_{\phi} \\
\mathbf{M}_{e 1 n}= & -\operatorname{sen} \phi \pi_{n}(\cos \theta) z_{n}(\rho) \hat{a}_{\theta}-\cos \phi \tau_{n}(\cos \theta) z_{n}(\rho) \hat{a}_{\phi} \\
\mathbf{N}_{o 1 n}= & \operatorname{sen} \phi n(n+1) \operatorname{sen} \theta \pi_{n}(\cos \theta) \frac{z_{n}(\rho)}{\rho} \hat{a}_{r}-\operatorname{sen} \phi \tau_{n}(\cos \theta) \frac{\left[\rho z_{n}(\rho)\right]^{\prime}}{\rho} \hat{a}_{\theta} \\
& +\cos \phi \pi_{n}(\cos \theta) \frac{\left[\rho z_{n}(\rho)\right]^{\prime}}{\rho} \hat{a}_{\phi} \\
\mathbf{N}_{e 1 n}= & \cos \phi n(n+1) \operatorname{sen} \theta \pi_{n}(\cos \theta) \frac{z_{n}(\rho)}{\rho} \hat{a}_{r}-\cos \phi \tau_{n}(\cos \theta) \frac{\left[\rho z_{n}(\rho)\right]^{\prime}}{\rho} \hat{a}_{\theta} \\
& +\operatorname{sen} \phi \pi_{n}(\cos \theta) \frac{\left[\rho z_{n}(\rho)\right]^{\prime}}{\rho} \hat{a}_{\phi} \\
& \pi_{n}(\cos \theta)=\frac{P_{n}^{1}(\cos \theta)}{\operatorname{sen} \theta} \quad \tau_{n}(\cos \theta)=\frac{d P_{n}^{1}(\cos \theta)}{d \theta}
\end{aligned}
$$

donde $\rho=k r$. A partir de las definiciones de los campos eléctricos y magnéticos en cada una de las regiones, las eficiencias de scattering y extinción (absorción mas scattering), se escriben como [13-17]:

$$
Q_{\text {scat }}=\frac{2}{k^{2} R_{3}^{2}} \sum_{n=1}^{\infty}(2 n+1)\left(\left|a_{n}\right|^{2}+\left|b_{n}\right|^{2}\right)
$$




$$
Q_{e x t}=\frac{2}{k^{2} R_{3}^{2}} \sum_{n=1}^{\infty}(2 n+1) \operatorname{Re}\left(a_{n}+b_{n}\right)
$$

La continuidad de los campos en cada una de las interfaces de los medios, producen las condiciones

$$
\begin{array}{rlr}
\left(\mathbf{E}_{2}-\mathbf{E}_{1}\right) \times \hat{a}_{r}=\left(\mathbf{H}_{2}-\mathbf{H}_{1}\right) \times \hat{a}_{r}=0 & r=R_{1} \\
\left(\mathbf{E}_{3}-\mathbf{E}_{2}\right) \times \hat{a}_{r}=\left(\mathbf{H}_{3}-\mathbf{H}_{2}\right) \times \hat{a}_{r}=0 & r=R_{2} \\
\left(\mathbf{E}_{i}+\mathbf{E}_{s}-\mathbf{E}_{3}\right) \times \hat{a}_{r}=\left(\mathbf{H}_{i}+\mathbf{H}_{s}-\mathbf{H}_{3}\right) \times \hat{a}_{r}=0 & r=R_{3}
\end{array}
$$

condiciones que al ser aplicadas, conducen al sistema de ecuaciones:

$$
\begin{aligned}
f_{n} m_{1} \psi_{n}\left(m_{2} x\right)-v_{n} m_{1} \chi_{n}\left(m_{2} x\right)-c_{n} m_{2} \psi_{n}\left(m_{1} x\right) & =0 \\
\mu_{1} f_{n} \psi_{n}^{\prime}\left(m_{2} x\right)-\mu_{1} v_{n} \chi_{n}^{\prime}\left(m_{2} x\right)-\mu_{2} c_{n} \psi_{n}^{\prime}\left(m_{1} x\right) & =0 \\
g_{n} m_{1} \psi_{n}^{\prime}\left(m_{2} x\right)-w_{n} m_{1} \chi_{n}^{\prime}\left(m_{2} x\right)-d_{n} m_{2} \psi_{n}^{\prime}\left(m_{1} x\right) & =0 \\
\mu_{1} g_{n} \psi_{n}\left(m_{2} x\right)-\mu_{1} w_{n} \chi_{n}\left(m_{2} x\right)-\mu_{2} d_{n} \psi_{n}\left(m_{1} x\right) & =0
\end{aligned}
$$

$$
\begin{aligned}
l_{n} m_{2} \psi_{n}\left(m_{3} y\right)-p_{n} m_{2} \chi_{n}\left(m_{3} y\right)-f_{n} m_{3} \psi_{n}\left(m_{2} y\right)+v_{n} m_{3} \chi_{n}\left(m_{2} y\right) & =0 \\
\mu_{2} l_{n} \psi_{n}^{\prime}\left(m_{3} y\right)-\mu_{2} p_{n} \chi_{n}^{\prime}\left(m_{3} y\right)-\mu_{3} f_{n} \psi_{n}^{\prime}\left(m_{2} y\right)+\mu_{3} v_{n} \chi_{n}^{\prime}\left(m_{2} y\right) & =0 \\
o_{n} m_{2} \psi_{n}^{\prime}\left(m_{3} y\right)-q_{n} m_{2} \chi_{n}^{\prime}\left(m_{3} y\right)-g_{n} m_{3} \psi_{n}^{\prime}\left(m_{2} y\right)+w_{n} m_{3} \chi_{n}^{\prime}\left(m_{2} y\right) & =0 \\
\mu_{2} o_{n} \psi_{n}\left(m_{3} y\right)-\mu_{2} q_{n} \chi_{n}\left(m_{3} y\right)-\mu_{3} g_{n} \psi_{n}\left(m_{2} y\right)+\mu_{3} w_{n} \chi_{n}\left(m_{2} y\right) & =0
\end{aligned}
$$

$$
\begin{aligned}
m_{3} \psi_{n}^{\prime}(z)-a_{n} m_{3} \xi_{n}^{\prime}(z)-o_{n} \psi_{n}^{\prime}\left(m_{3} z\right)-q_{n} \chi_{n}^{\prime}\left(m_{3} z\right) & =0 \\
\mu_{3} \psi_{n}(z)-\mu_{3} a_{n} \xi_{n}(z)-\mu o_{n} \psi_{n}\left(m_{3} z\right)+\mu q_{n} \chi_{n}\left(m_{3} z\right) & =0 \\
b_{n} m_{3} \xi_{n}(z)-m_{3} \psi_{n}(z)+l_{n} \psi_{n}\left(m_{3} z\right)-p_{n} \chi_{n}\left(m_{3} z\right) & =0 \\
\mu_{3} b_{n} \xi_{n}^{\prime}(z)-\mu_{3} \psi_{n}^{\prime}(z)+\mu l_{n} \psi_{n}^{\prime}\left(m_{3} z\right)-\mu p_{n} \chi_{n}^{\prime}\left(m_{3} z\right) & =0,
\end{aligned}
$$


donde $m_{1}=k_{1} / k=N_{1} / N, m_{2}=k_{2} / k=N_{2} / N, m_{3}=k_{3} / k=N_{3} / N, k_{i}=2 \pi N_{i} / \lambda$, $x=k R_{1}, y=k R_{2}$ y $z=k R_{3}$. Adicionalmente se definen las funciones $\psi_{n}(\rho)=\rho j_{n}(\rho)$, $\chi_{n}(\rho)=-\rho y_{n}(\rho)$ y $\xi_{n}(\rho)=\rho h_{n}^{(1)}(\rho)$, cuyas gráficas, se muestran en las Figuras B.3 y B.4.

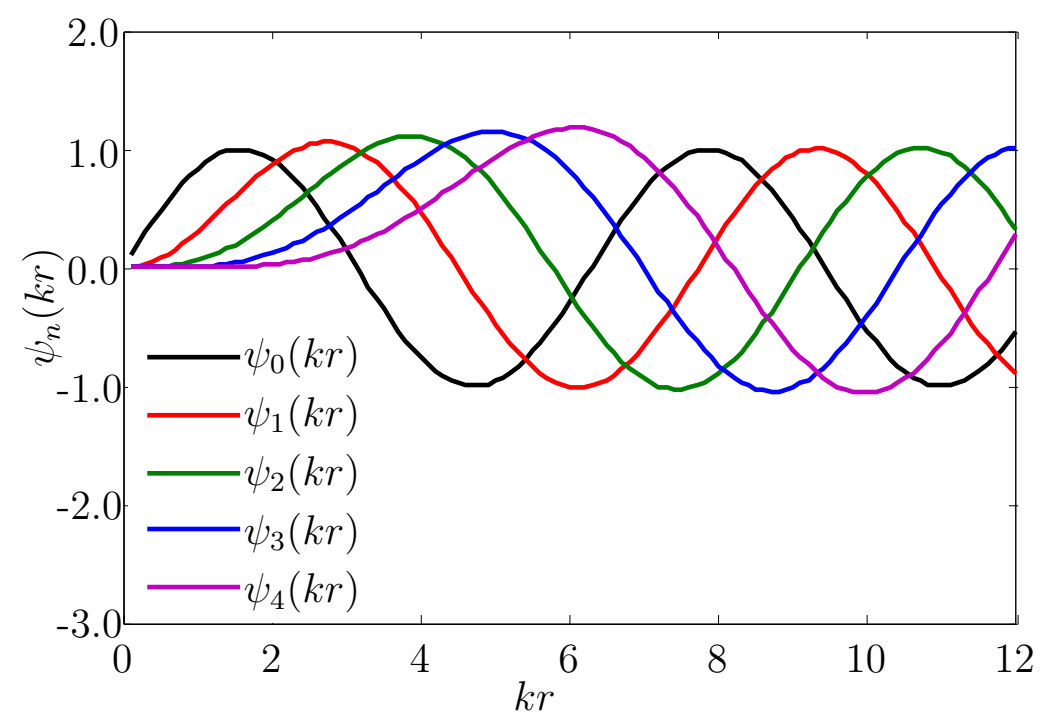

Figura B.3: Gráfica de la función $\psi_{n}(\rho)=\rho j_{n}(\rho)$, en términos de $\rho=k r$

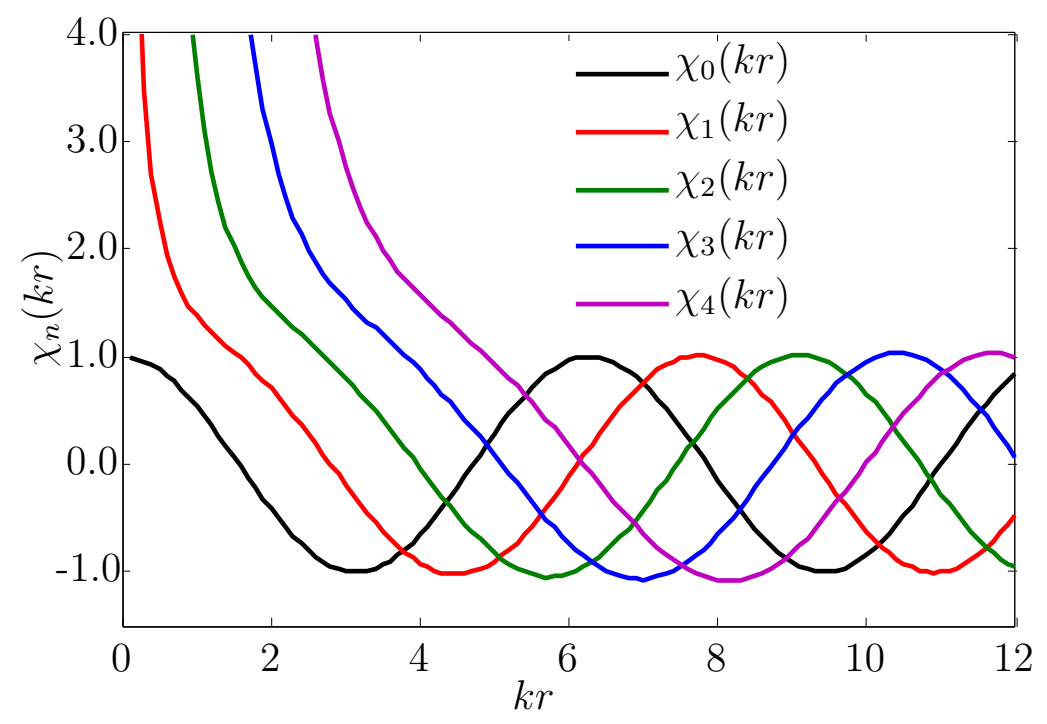

Figura B.4: Gráfica de la función $\chi_{n}(\rho)=-\rho y_{n}(\rho)$, en términos de $\rho=k r$ 
Los coeficientes $a_{n}$ y $b_{n}$ que definen las eficiencias de absorción y extinción, se obtienen de la solución al sistema de ecuaciones como:

$$
\begin{aligned}
a_{n} & =\frac{\mu m_{3} \psi_{n}^{\prime}(z)\left[\psi_{n}\left(m_{3} z\right)-A_{n} \chi_{n}\left(m_{3} z\right)\right]-\mu_{3} \psi_{n}(z)\left[\psi_{n}^{\prime}\left(m_{3} z\right)-A_{n} \chi_{n}^{\prime}\left(m_{3} z\right)\right]}{\mu m_{3} \xi_{n}^{\prime}(z)\left[\psi_{n}\left(m_{3} z\right)-A_{n} \chi_{n}\left(m_{3} z\right)\right]-\mu_{3} \xi_{n}(z)\left[\psi_{n}^{\prime}\left(m_{3} z\right)-A_{n} \chi_{n}^{\prime}\left(m_{3} z\right)\right]} \\
b_{n} & =\frac{\mu m_{3} \psi_{n}(z)\left[\psi_{n}^{\prime}\left(m_{3} z\right)-B_{n} \chi_{n}^{\prime}\left(m_{3} z\right)\right]-\mu_{3} \psi_{n}^{\prime}(z)\left[\psi_{n}\left(m_{3} z\right)-B_{n} \chi_{n}\left(m_{3} z\right)\right]}{\mu m_{3} \xi_{n}(z)\left[\psi_{n}^{\prime}\left(m_{3} z\right)-B_{n} \chi_{n}^{\prime}\left(m_{3} z\right)\right]-\mu_{3} \xi_{n}^{\prime}(z)\left[\psi_{n}\left(m_{3} z\right)-B_{n} \chi_{n}\left(m_{3} z\right)\right]}
\end{aligned}
$$

donde

$$
\begin{aligned}
A_{n} & =\frac{\mu_{2} m_{3} \psi_{n}\left(m_{3} y\right)\left[\psi_{n}^{\prime}\left(m_{2} y\right)-M_{n} \xi_{n}^{\prime}\left(m_{2} y\right)\right]-\mu_{3} m_{2} \psi_{n}^{\prime}\left(m_{3} y\right)\left[\psi_{n}\left(m_{2} y\right)-M_{n} \xi_{n}\left(m_{2} y\right)\right]}{\mu_{2} m_{3} \chi_{n}\left(m_{3} y\right)\left[\psi_{n}^{\prime}\left(m_{2} y\right)-M_{n} \xi_{n}^{\prime}\left(m_{2} y\right)\right]-\mu_{3} m_{2} \chi_{n}^{\prime}\left(m_{3} y\right)\left[\psi_{n}\left(m_{2} y\right)-M_{n} \xi_{n}\left(m_{2} y\right)\right]} \\
B_{n} & =\frac{\mu_{2} m_{3} \psi_{n}^{\prime}\left(m_{3} y\right)\left[\psi_{n}\left(m_{2} y\right)-N_{n} m_{3} \xi_{n}\left(m_{2} y\right)\right]-\mu_{3} m_{2} \psi_{n}\left(m_{3} y\right)\left[\psi_{n}^{\prime}\left(m_{2} y\right)-N_{n} \xi_{n}^{\prime}\left(m_{2} y\right)\right]}{\mu_{2} m_{3} \chi_{n}^{\prime}\left(m_{3} y\right)\left[\psi_{n}\left(m_{2} y\right)-N_{n} m_{3} \xi_{n}\left(m_{2} y\right)\right]-\mu_{3} m_{2} \chi_{n}\left(m_{3} y\right)\left[\psi_{n}^{\prime}\left(m_{2} y\right)-N_{n} \xi_{n}^{\prime}\left(m_{2} y\right)\right]}
\end{aligned}
$$

$$
\begin{aligned}
M_{n} & =\frac{\mu_{1} m_{2} \psi_{n}\left(m_{2} x\right) \psi_{n}^{\prime}\left(m_{1} x\right)-\mu_{2} m_{1} \psi_{n}\left(m_{1} x\right) \psi_{n}^{\prime}\left(m_{2} x\right)}{\mu_{1} m_{2} \chi_{n}\left(m_{2} x\right) \psi_{n}^{\prime}\left(m_{1} x\right)-\mu_{2} m_{1} \psi_{n}\left(m_{1} x\right) \chi_{n}^{\prime}\left(m_{2} x\right)} \\
N_{n} & =\frac{\mu_{1} m_{2} \psi_{n}\left(m_{1} x\right) \psi_{n}^{\prime}\left(m_{2} x\right)-\mu_{2} m_{1} \psi_{n}^{\prime}\left(m_{1} x\right) \psi_{n}\left(m_{2} x\right)}{\mu_{1} m_{2} \chi_{n}^{\prime}\left(m_{2} x\right) \psi_{n}\left(m_{1} x\right)-\mu_{2} m_{1} \psi_{n}^{\prime}\left(m_{1} x\right) \chi_{n}\left(m_{2} x\right)}
\end{aligned}
$$

\section{B.3. Nanopartículas esféricas con una cubierta}

NPs más sencillas de estudiar corresponden a las de estructura núcleo-cubierta o core-shell Figura B.5. Para obtener las expresiones de los coeficientes para estás NPs, realizamos la simplificación $m_{2}=m_{1}$ y $\mu_{2}=\mu_{1}$, obteniendo en este caso que $M_{n}=$ $N_{n}=0$. Para estas NPs los coeficientes $A_{n}$ y $B_{n}$ se simplifican en [4, 13, 14, 166, 167]:

$$
\begin{aligned}
A_{n} & =\frac{\mu_{1} m_{3} \psi_{n}\left(m_{3} y\right) \psi_{n}^{\prime}\left(m_{1} y\right)-\mu_{3} m_{1} \psi_{n}^{\prime}\left(m_{3} y\right) \psi_{n}\left(m_{1} y\right)}{\mu_{1} m_{3} \chi_{n}\left(m_{3} y\right) \psi_{n}^{\prime}\left(m_{1} y-\mu_{3} m_{1} \chi_{n}^{\prime}\left(m_{3} y\right) \psi_{n}\left(m_{1} y\right)\right.} \\
B_{n} & =\frac{\mu_{1} m_{3} \psi_{n}^{\prime}\left(m_{3} y\right) \psi_{n}\left(m_{1} y\right)-\mu_{3} m_{1} \psi_{n}\left(m_{3} y\right) \psi_{n}^{\prime}\left(m_{1} y\right)}{\mu_{1} m_{3} \chi_{n}^{\prime}\left(m_{3} y\right) \psi_{n}\left(m_{1} y\right)-\mu_{3} m_{1} \chi_{n}\left(m_{3} y\right) \psi_{n}^{\prime}\left(m_{1} y\right)}
\end{aligned}
$$




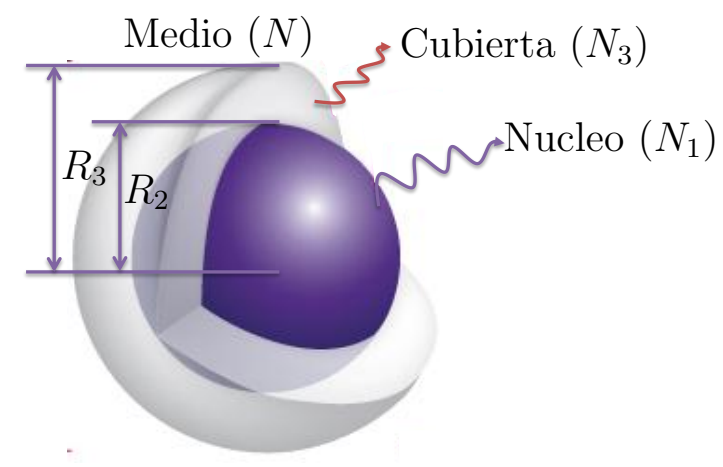

Figura B.5: Nanopartícula núcleo-cubierta o core shell inmersa en un medio de indice $N$, de núcleo con radio $R_{2}$ y cubierta $R_{3}$ con indices $N_{1}$ y $N_{3}$ respectivamente

\section{B.4. Nanopartículas esféricas sin cubierta}

Las NPs más simples corresponden a un núcleo desnudo. En este caso las expresiones de los coeficientes de Mie se pueden obtener de los coeficientes que describen las NPs núcleo-cubierta con la consideración $m_{3}=m_{1}=m$ y $\mu_{3}=\mu_{1}=\mu$, en este

caso es sencillo ver que $A_{n}=B_{n}=0 \mathrm{y}$ los coeficientes $a_{n}$ y $b_{n}$, se simplifican como 4, 13, 14, 166, 167:

$$
\begin{aligned}
a_{n} & =\frac{\mu m \psi_{n}^{\prime}(z) \psi_{n}(m z)-\mu_{1} \psi_{n}(z) \psi_{n}^{\prime}(m z)}{\mu m \xi_{n}^{\prime}(z) \psi_{n}(m z)-\mu_{1} \xi_{n}(z) \psi_{n}^{\prime}(m z)} \\
b_{n} & =\frac{\mu m \psi_{n}(z) \psi_{n}^{\prime}(m z)-\mu_{1} \psi_{n}^{\prime}(z) \psi_{n}(m z)}{\mu m \xi_{n}(z) \psi_{n}^{\prime}(m z)-\mu_{1} \xi_{n}^{\prime}(z) \psi_{n}(m z)}
\end{aligned}
$$

\section{B.5. Cálculo de los coeficientes de Mie}

Para facilitar la programación en el cálculo de los coeficientes $a_{n}$ y $b_{n}$ y mejorar la convergencia de las series, las expresiones B.42, B.47, con $\mu_{1}=\mu_{2}=\mu_{3}=\mu=1$, se pueden escribir de forma alternativa como:

$$
a_{n}=\frac{\psi_{n}(z)\left[\widetilde{A}_{n} / m_{3}+n / z\right]-\psi_{n-1}(z)}{\xi_{n}(z)\left[\widetilde{A}_{n} / m_{3}+n / z\right]-\xi_{n-1}(z)}
$$




$$
\begin{gathered}
b_{n}=\frac{\psi_{n}(z)\left[m_{3} \widetilde{B}_{n}+n / z\right]-\psi_{n-1}(z)}{\xi_{n}(z)\left[m_{3} \widetilde{B}_{n}+n / z\right]-\xi_{n-1}(z)} \\
\widetilde{A}_{n}=\frac{D_{n}\left(m_{3} z\right)-A_{n} \chi_{n}^{\prime}\left(m_{3} z\right) / \psi_{n}\left(m_{3} z\right)}{1-A_{n} \chi_{n}\left(m_{3} z\right) / \psi_{n}\left(m_{3} z\right)} \\
\widetilde{B}_{n}=\frac{D_{n}\left(m_{3} z\right)-B_{n} \chi_{n}^{\prime}\left(m_{3} z\right) / \psi_{n}\left(m_{3} z\right)}{1-B_{n} \chi_{n}\left(m_{3} z\right) / \psi_{n}\left(m_{3} z\right)} \\
A_{n}=\frac{\psi_{n}\left(m_{3} y\right)\left[m_{32} \widetilde{M}_{n}-D_{n}\left(m_{3} y\right)\right]}{m_{32} \chi_{n}\left(m_{3} y\right) \widetilde{M}_{n}-\chi_{n}^{\prime}\left(m_{3} y\right)} \\
N_{n}=\frac{\psi_{n}\left(m_{3} y\right)\left[\widetilde{N}_{n} / m_{32}-D_{n}\left(m_{3} y\right)\right]}{\chi_{n}\left(m_{3} y\right) \widetilde{M}_{n} / m_{32}-\chi_{n}^{\prime}\left(m_{3} y\right)} \\
\mathcal{B}_{n}\left(m_{2} x\right) \frac{m_{21} D_{n}\left(m_{2} x\right)-D_{n}\left(m_{1} x\right)}{m_{21} \chi_{n}^{\prime}\left(m_{2} x\right)-D_{n}\left(m_{1} x\right) \chi_{n}\left(m_{2} x\right)} \\
\widetilde{M}_{n}=\frac{D_{n}\left(m_{2} y\right) \psi_{n}\left(m_{2} y\right)-M_{n} \chi_{n}^{\prime}\left(m_{2} y\right)}{\psi_{n}\left(m_{2} y\right)-M_{n} \chi_{n}\left(m_{2} y\right)} \\
\widetilde{D}_{n}\left(m_{2} y\right) \psi_{n}\left(m_{2} y\right)-N_{n} \chi_{n}^{\prime}\left(m_{2} y\right) \\
\left.\psi_{n}\left(m_{2} y\right)-m_{n} x\right)-D_{n}\left(m_{2} x\right)
\end{gathered}
$$

donde $m_{21}=m_{2} / m_{1}, m_{32}=m_{3} / m_{2}, D_{n}=d \ln \psi_{n}(\rho) / d \rho$, y además a partir de las formulas de recurrencia de las funciones de Bessel, se pueden obtener funciones de recurrencia para $D_{n}$ y las derivadas $\chi_{n}^{\prime}$ y $\xi_{n}^{\prime}[\mathbf{1 3}]$.

$$
D_{n-1}=\frac{n}{\rho}-\frac{1}{D_{n}+n / \rho}, \quad \chi_{n}^{\prime}=\chi_{n} D_{n}-\frac{1}{\psi_{n}}, \quad \xi_{n}^{\prime}=\xi_{n} D_{n}+\frac{i}{\psi_{n}},
$$

debido a que la relación de recurrencia para $D_{n}$, presenta mejor convergencia si se realiza en forma decreciente, se debe tomar un valor límite $N$ donde se considera cero dicha función y a partir de este valor se calculan los $N-1$ valores anteriores; este límite se escoge de tal forma que $N$ sea mayor que el modulo de $z+4 z^{1 / 3}+2[13]$. 


\section{B.6. Aproximación de Mie para nanopartículas pequeñas}

Para NPs pequeñas comparadas con la longitud de onda, los términos de orden superior de la serie de Mie son despreciables y se puede consider solo el primero de los términos de la serie; tomando las funciones de Bessel esféricas, para ordenes de z menores de 4 y $n=1$

$$
\begin{aligned}
& \psi_{1}(z)=\frac{z^{2}}{3}+O\left(z^{4}\right) \\
& \xi_{1}(z)=\frac{-i}{z}-\frac{i z}{2}+\frac{z^{3}}{3}+O\left(z^{4}\right) \\
& \chi_{1}(z)=\frac{-1}{z}-\frac{z}{2}+O\left(z^{4}\right)
\end{aligned}
$$

si solo se toman en cuenta los términos de $z$ inferiores al cuarto orden, los coeficientes de Mie definidos por las ecuaciones B.42 B.47 se aproximan a

$$
\begin{gathered}
a_{1} \approx \frac{\frac{1}{3} A_{1}\left(2 m_{3}^{2}+1\right)+\frac{2}{9} m_{3}^{3} z^{3}\left(m_{3}^{2}-1\right)}{\frac{A_{1}}{3}\left(m_{3}^{2}-1\right)+\frac{1}{3} m_{3}^{3}\left(m_{3}^{2}+2\right)} \quad b_{1} \approx 0 \\
A_{1} \approx \frac{\frac{2}{9} m_{2}^{3} m_{3}^{3}\left(m_{3}^{2}-m_{2}^{2}\right) y^{3}-\frac{1}{3} m_{3}^{3} M_{1}\left(m_{3}^{2}+2 m_{2}^{2}\right)}{-\frac{1}{3} m_{2}^{3}\left(2 m_{3}^{2}+m_{2}^{2}\right) y^{3}+\frac{M_{1}}{y^{3}}\left(m_{3}^{2}-m_{2}^{2}\right)} \quad B_{1} \approx \frac{m_{3}^{3}}{m_{2}^{3}} N_{1} \\
N_{1} \approx \frac{1}{90} m_{2}^{3}\left(m_{1}^{2}-m_{2}^{2}\right) x^{5} \quad M_{1} \approx-\frac{2}{3} m_{2}^{3} \frac{\left(m_{2}^{2}-m_{1}^{2}\right)}{\left(2 m_{2}^{2}-m_{1}^{2}\right)} x^{3},
\end{gathered}
$$

remplazando cada uno de los términos y utilizando el hecho de que la sección eficaz

de extinción es $C_{e x t}=\frac{6 \pi}{k^{2}} \operatorname{Real}\left\{a_{1}\right\}=k \operatorname{Imag}\{\alpha\}$, donde $\alpha$ es la polarizabilidad de la NP se tiene 133, 134 


$$
\alpha=4 \pi c^{3} \frac{\alpha_{1}+\alpha_{2}}{\alpha_{3}+\alpha_{4}}
$$

donde

$$
\begin{aligned}
& \alpha_{1}=\left(\varepsilon_{1}+2 \varepsilon_{2}\right)\left(\varepsilon_{2}-\varepsilon_{3}\right)\left(2 \varepsilon_{3}+\varepsilon_{m}\right) f+\left(2 \varepsilon_{2}+\varepsilon_{1}\right)\left(2 \varepsilon_{3}+\varepsilon_{2}\right)\left(\varepsilon_{3}-\varepsilon_{m}\right) \\
& \alpha_{2}=\left(\varepsilon_{2}-\varepsilon_{1}\right)\left(\varepsilon_{3}-\varepsilon_{2}\right)\left(2 \varepsilon_{3}-2 \varepsilon_{m}\right) g+\left(\varepsilon_{1}-\varepsilon_{2}\right)\left(\varepsilon_{3}+2 \varepsilon_{2}\right)\left(2 \varepsilon_{3}+\varepsilon_{m}\right) h\left(\varepsilon_{1}\right)\left(2 \varepsilon_{2}\right)\left(\varepsilon_{2}-\varepsilon_{3}\right)\left(2 \varepsilon_{3}-2 \varepsilon_{m}\right) f+\left(2 \varepsilon_{2}+\varepsilon_{1}\right)\left(2 \varepsilon_{3}+\varepsilon_{2}\right)\left(\varepsilon_{3}+2 \varepsilon_{m}\right)( \\
& \alpha_{3}=\left(\varepsilon_{1}+2 \varepsilon_{3}\right. \\
& \alpha_{4}=2\left(\varepsilon_{2}-\varepsilon_{1}\right)\left(\varepsilon_{3}-\varepsilon_{2}\right)\left(\varepsilon_{3}+2 \varepsilon_{m}\right) g+\left(\varepsilon_{1}-\varepsilon_{2}\right)\left(\varepsilon_{3}+2 \varepsilon_{2}\right)\left(2 \varepsilon_{3}-2 \varepsilon_{m}\right) h(B)
\end{aligned}
$$

$\varepsilon_{1}, \varepsilon_{2}$ y $\varepsilon_{3}$ son las funciones dieléctricas del núcleo y de cada una de las cubiertas de la NP, y $\varepsilon_{m}$ es la función dieléctrica del medio en el cual se encuentra la NP, $f=R_{2}^{3} / R_{3}^{3}, g=R_{1}^{3} / R_{2}^{3}$ y $h=R_{1}^{3} / R_{3}^{3}$. Cuando la NP posee una sola cubierta o son de núcleo desnudo, la expresión para la polarizabilidad se simplifica de acuerdo a las consideraciones geométricas mostradas en la Figura B.6.

Utilizando estos coeficientes de Mie, las eficiencias de scattering y extinción para el caso de NPs desnudas, muy pequeñas con respecto a la longitud de onda están aproximadas por $[\mathbf{1 3}, \mathbf{1 5}, \mathbf{1 6}, \mathbf{1 6 8}-\mathbf{1 7 0}$ :

$$
Q_{\text {scat }}=\frac{8}{3} z^{4}\left|\frac{\epsilon-\epsilon_{m}}{\epsilon+2 \epsilon_{m}}\right|^{2} \quad Q_{\text {ext }}=4 z \operatorname{Im}\left\{\frac{\epsilon-\epsilon_{m}}{\epsilon+2 \epsilon_{m}}\right\}
$$

donde $\epsilon \mathrm{y} \epsilon_{m}$, son las permitividades relativas de la NP y del medio respectivamente. Para que la aproximación dipolar se valida se debe cumplir la condición $1 /|m z| \ll 1$ que relaciona el tamaño de la NP con la longitud de onda y el medio, donde $m$ es la razón de índices entre la NP y el medio, $z=k R_{3}$ y $R_{3}$ es el radio de la NP.

En la Figura B.7 se muestra una gráfica de $1 /|m k|$ en términos de la longitud de onda para $\mathrm{Au}, \mathrm{Ag}$ y $\mathrm{Cu}$. Para encontrarse en la aproximación dipolar en estas NPs su radio debe ser inferior a $2.5 \mathrm{~nm}$ tomando el criterio $1 /|m z|=0,1 \ll 1$. 


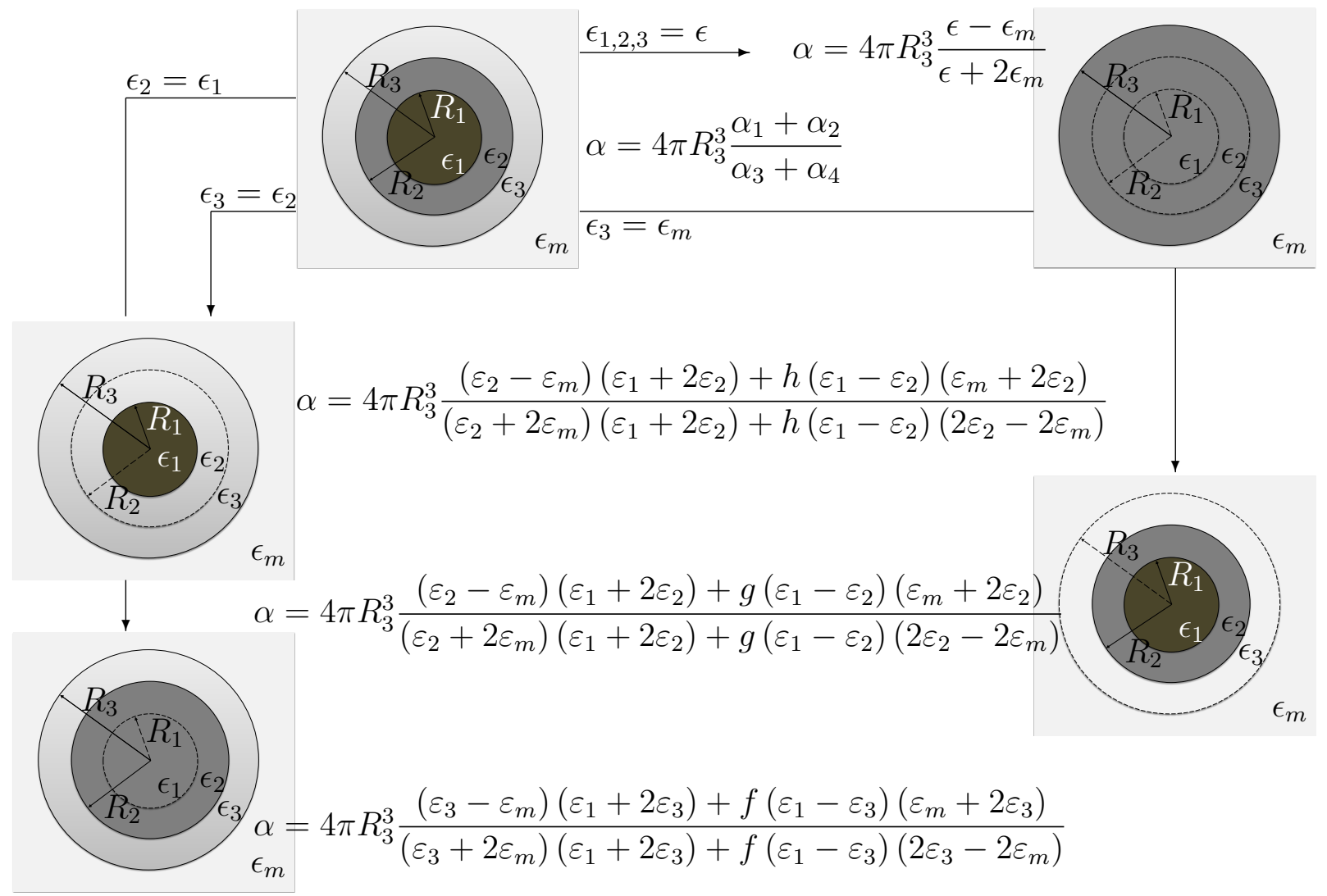

Figura B.6: Expresiones de la polarizabilidad para distintas configuiraciones geométricas y expresiones y estructuras.

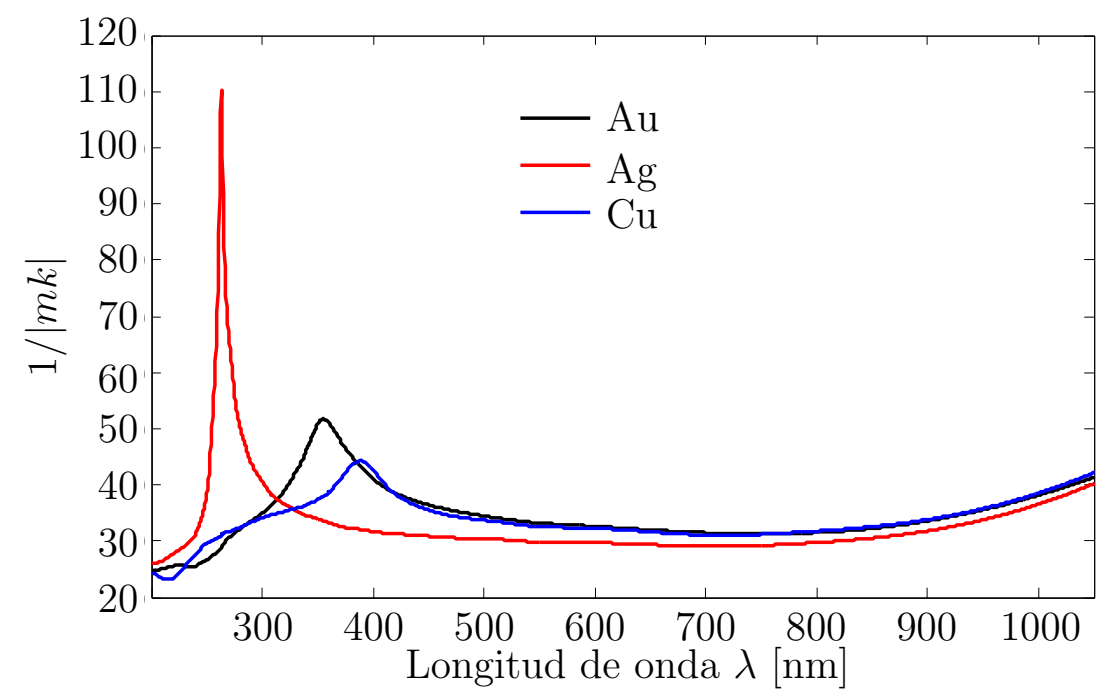

Figura B.7: Determinación del radio para el que una nanopartícula de $\mathrm{Au}, \mathrm{Ag}$ y $\mathrm{Cu}$, se encuentra en la aproximación dipolar. 
Si se aplica la aproximación dipolar a NPs de núcleo desnudo Fe y Al en el rango de 200-2000 nm de longitud de onda, esta aproximación no predice un plasmón. Esto ocurre porque la aproximación dipolar solo predice adecuadamente la ubicación del plasmón de NPs pequeñas y estas presentarían un pico plasmónico en longitudes de onda inferiores a 200 nm. Las NPs que presentan plasmón en longitudes de onda superiores a 200 nm se encuentran fuera del rango de validez de la aproximación dipolar, por tal motivo para NPs.

\section{B.7. Extinción de nanopartículas magnéticas}

La teoría de Mie es el formalismo más general para modelar la extinción óptica de NPs. La precisión de los resultados dependen del número de términos multipolares considerados en los cálculos. Para NPs no magnéticas se considera solamente la interacción eléctrica, por medio de la dependencia con la frecuencia de la permitividad $\epsilon(\omega)$, tomando la permeabilidad magnetica $\mu=1$.

Con la intensión de considerar los efectos magnéticos en la extinción, Draine \& Hensley presentan un modelo para NPs magnéticas (MNPs) en un medio interestelar, que consiste en adicionar un termino dipolar magnético a la teoría de Mie con $\mu=1$ [68]. Sin embargo, una descripción más precisa de la extinción requiere la consideración explícita de la contribución magnética por la dependencia con la frecuencia de la susceptibilidad $\mu(\omega)$.

Para el caso de NPs de materiales conductores, se deben considerar los efectos de las corrientes de Eddy, como muestran Draine \& Hensey en el trabajo citado. Estos efectos se vuelven importantes para un intervalo de tamaño de NPs y debe determinarse para cada material en comparación con modelos que no incluyen las corrientes de Eddy.

Si consideramos el volumen de una NP como $V$, el momento dipolar magnético se define como:

$$
\mathbf{p}_{m}=V \mathbf{m}=\alpha_{m} \cdot \mathbf{h}
$$

donde $\alpha_{m}$ es el tensor de polarizabilidad magnética que se puede describir como 
la suma de dos contribuciones la magnética $\alpha^{m a g}$ y la polarizabilidad debida a las corrientes de Eddy $\alpha^{E d d y}$. En el caso de no considerar las corrientes de Eddy se describe como:

$$
\alpha_{m}^{s}=V\left(\chi_{+} \mathbf{l}_{+} \mathbf{l}_{+}^{*}+\chi_{-} \mathbf{l}_{-} \mathbf{l}_{-}^{*}\right)
$$

Es importante tener en cuenta que para materiales conductores se debe considerar la contribución de las corrientes de Eddy. La contribución de las corrientes de eddy para una esfera de radio $R$ para un medio no magnético [171], que es un tensor diagonal con elementos iguales a

$$
\alpha^{E d d y}=\frac{3 V}{8 \pi}\left[\frac{3}{y^{2}}-\frac{3}{y} \cot y-1\right]
$$

donde $y^{2}=\varepsilon \omega^{2} R^{2} / c^{2}$. Para una conductividad finita, las corrientes de eddy no se encuentran confinadas en la superficie y el campo magnético en el interior es no uniforme, un valor típico del campo en el interior es [68]

$$
\begin{aligned}
\mathbf{h}_{e f f} & \approx \mathbf{h}_{0}\left(1-\phi_{E d d y}\right) e^{-i \omega t} \\
\phi_{E d d y} & =-\frac{8 \pi \alpha^{E d d y}}{3 V}
\end{aligned}
$$

Con lo anterior el tensor de polarizabilidad magnética es:

$$
\alpha^{m a g}=\left(1-\phi_{E d d y}\right) \alpha_{m}^{s}=\left(1-\phi_{E d d y}\right) V\left(\chi_{+} \mathbf{h}_{+} \mathbf{h}_{+}^{*}+\chi_{-} \mathbf{h}_{-} \mathbf{h}_{-}^{*}\right)
$$

Cuando nos encontramos en presencia de un conductor perfecto $\operatorname{Im}(y)$ tiende a infinito lo que implica que $\alpha^{E d d y}$ tiende a $-\frac{3 V}{8 \pi}$ y $\phi_{E d d y}$ tiende a 1 en cuyo caso el campo magnético generado por las corrientes de Eddy se anula completamente dentro de la esfera. Para el caso de un no conductor $\phi_{E d d y}=0$ y $\alpha_{m}=\alpha^{m a g}$. Los efectos magnéticos en NPs aparecen para frecuencias inferiores a $600 \mathrm{GHz}$ (longitudes de onda mayores a $500 \mu \mathrm{m}$ ) por tal motivo la contribución magnética a la extinción se puede considerar como dipolar. En términos generales la extinción es la suma de la extinción 
magnética (dipolar) y la extinción eléctrica (Teoría de mie).

$$
C_{e x t}=C_{e x t}^{T M}+\frac{4 \pi \omega V}{3 c} \operatorname{Im}\left[\left(1-\phi_{E d d y}\right)\left(\chi_{+}+\chi_{-}\right)\right]+\frac{4 \pi \omega}{c} \operatorname{Im}\left[\alpha_{E d d y}\right]
$$

La Figura B.8 muestra los resultados de eficiencia de extinción correspondiente a las diferentes aproximaciones mencionadas anteriormente, para varios tamaños de NPs. La teoría de Mie con permeabilidad constante $\mu=1\left(Q_{e x t}^{M i e}(\mu=1)\right)$ y la teoría de Mie con permeabilidad dependiente de la frecuencia $\mu(\omega)\left(Q_{e x t}^{M i e}(\mu(\omega))\right)$, se comparan con la teoría de Mie con $\mu$ constante más la contribución dipolar magnética (considerada como la suma de las contribuciones magnéticas y Eddy) y la teoría de Mie con $\mu(\omega)$ más la contribución de Eddy.

Como muestra la Figura B.8, la consideración de susceptibilidad dependiente de la frecuencia se vuelve importante para las longitudes de onda largas (más largas para las NPs más grandes), pero puede ser reemplazada por la corrección dipolar magnética con resultados idénticos. Sólo el caso $\mu=1$ sin curva de corrección dipolar magnética (línea punteada negra) presenta un comportamiento separado para longitudes de onda largas.

Las corrientes de Eddy, por el contrario, son irrelevantes para NPs pequeñas $(R<10 \mathrm{~nm})$, mientras que para NPs grandes se vuelven importantes a longitudes de onda cortas como se muestra en la sección ampliada de la Figura B.8, donde se incluyen las corrientes de Eddy en los modelos (líneas azules y rosadas). Todos estos resultados se resumen en la Tabla B.1.

La relevancia de las corrientes de Eddy para NPs con radios superiores a $20 \mathrm{~nm}$, deben tenerse en cuenta también para los sistemas multicapa. Los cálculos se pueden realizar como una extensión del método desarrollado por Landau. Denominando $\epsilon_{i}$, $i=1,2,3$ la permitividad para cada una de las capas y los radios $R_{1}, R_{2}$ y $R_{3}$ con $R_{1}<R_{2}<R_{3}$, después de resolver las ecuaciones de Maxwell, los campos magnéticos en cada una de las regiones dependen de la dirección del campo eléctrico externo e: [171 


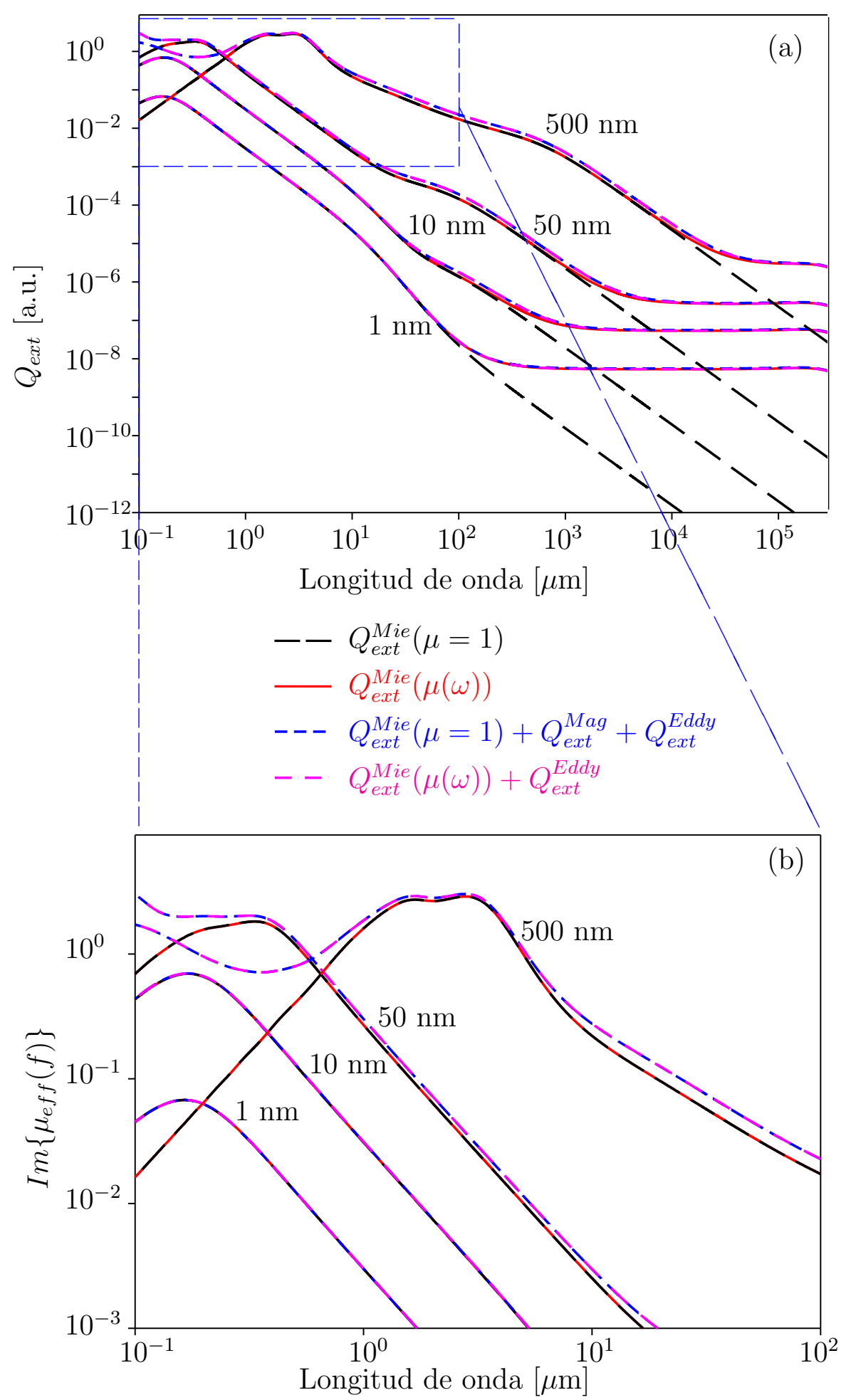

Figura B.8: Comparación entre diferentes aproximaciones para el cálculo de la extinción de NPs de diferentes tamaños. 


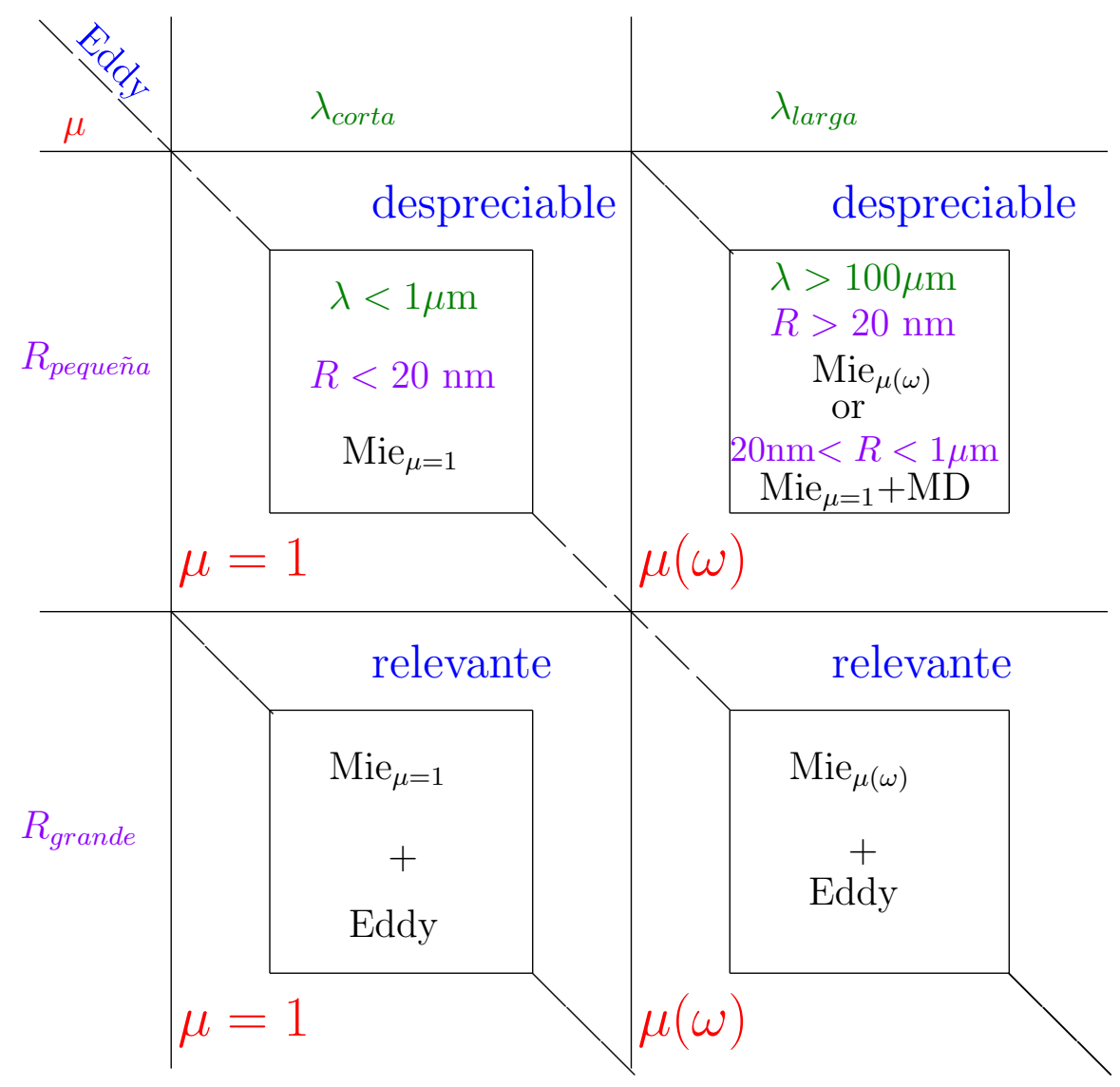

Tabla B.1: Resumen de la extinción óptica para diferentes rangos de longitud de onda y diferentes tamaños de NPs

$$
\mathbf{H}_{i}=\left(\frac{f_{i}^{\prime}(r)}{r}+k_{i}^{2} f_{i}(r)\right) \mathbf{e}-\left(\frac{3 f_{i}^{\prime}(r)}{r}+k_{i}^{2} f_{i}(r)\right)(\mathbf{n} \cdot \mathbf{e}) \mathbf{n}
$$

para $i=1,2,3$

$$
\mathbf{H}_{e}=\frac{\alpha}{r^{3}}[3(\mathbf{n} \cdot \mathbf{e}) \mathbf{n}-\mathbf{e}]+\mathbf{e}
$$

donde $f_{1}=\beta_{1} \operatorname{sen} k_{1} r / r, f_{2}=\beta_{2} \operatorname{sen} k_{2} r / r+\beta_{3} \cos k_{2} r / r, f_{3}=\beta_{4} \operatorname{sen} k_{3} r / r+\beta_{5} \cos k_{3} r / r$, $f_{j}^{\prime}(r)=d f_{j}(r) / d r$ y $\alpha$ es la polarizabilidad. Las condiciones de continuidad para los campos en $r=R_{1}$ son 


$$
\begin{aligned}
\frac{f_{1}^{\prime}\left(R_{1}\right)}{R_{1}}+k_{1}^{2} f_{1}\left(R_{1}\right) & =\frac{f_{2}^{\prime}\left(R_{1}\right)}{R_{1}}+k_{2}^{2} f_{2}\left(R_{1}\right) \\
\frac{3 f_{1}^{\prime}\left(R_{1}\right)}{R_{1}}+k_{1}^{2} f_{1}\left(R_{1}\right) & =\frac{3 f_{2}^{\prime}\left(R_{1}\right)}{R_{1}}+k_{2}^{2} f_{2}\left(R_{1}\right)
\end{aligned}
$$

para $r=R_{2}$

$$
\begin{aligned}
\frac{f_{2}^{\prime}\left(R_{2}\right)}{R_{2}}+k_{2}^{2} f_{2}\left(R_{2}\right) & =\frac{f_{3}^{\prime}\left(R_{2}\right)}{R_{2}}+k_{3}^{2} f_{3}\left(R_{2}\right) \\
\frac{3 f_{2}^{\prime}\left(R_{2}\right)}{R_{2}}+k_{2}^{2} f_{2}\left(R_{2}\right) & =\frac{3 f_{3}^{\prime}\left(R_{2}\right)}{R_{2}}+k_{3}^{2} f_{3}\left(R_{2}\right)
\end{aligned}
$$

y finalmente para $r=R_{3}$

$$
\begin{aligned}
\frac{f_{3}^{\prime}\left(R_{3}\right)}{R_{3}}+k_{3}^{2} f_{3}\left(R_{3}\right) & =1-\frac{\alpha}{R_{3}^{3}} \\
\frac{3 f_{3}^{\prime}\left(R_{3}\right)}{R_{3}}+k_{3}^{2} f_{3}\left(R_{3}\right) & =-\frac{3 \alpha}{R_{3}^{3}} .
\end{aligned}
$$

Combinando estas condiciones, la polarizabilidad $\alpha$ se puede obtener:

$$
\begin{array}{r}
\alpha=-\frac{3 V}{8 \pi} \frac{\beta_{45}\left(z^{2} \tan z+3 z-3 \tan z\right)+\left(z^{2}-3 z \tan z-3\right)}{\beta_{45} z^{2} \tan z+z^{2}} \\
\beta_{45}=\frac{\left(y_{2}^{2}-y_{3}^{2}+y_{2}^{2} y_{3} \tan y_{3}\right)\left(1+\beta_{23} \tan y_{2}\right)+y_{2} y_{3}^{2}\left(\beta_{23}-\tan y_{2}\right)}{\left(y_{3}^{2}-y_{2}^{2}+y_{2} y_{3}^{2}\right)\left(1+\beta_{23} \tan y_{2}\right)+y_{2}^{2} y_{3} \tan y_{2}\left(\beta_{23}+\tan y_{3}\right)} \\
\beta_{23}=\frac{x_{1}^{2} x_{2} \tan x_{1} \tan x_{2}+\left(x_{1}^{2}-x_{2}^{2}\right) \tan x_{1}+x_{1} x_{2}^{2}}{\left(x_{2}^{2}-x_{1}^{2}\right) \tan x_{1} \tan x_{2}+x_{1}^{2} x_{2} \tan x_{1}-x_{1} x_{2}^{2} \tan x_{2}}
\end{array}
$$

donde $x_{j}^{2}=\varepsilon_{j}\left(\omega R_{1} / c\right)^{2}, y_{j}^{2}=\varepsilon_{j}\left(\omega R_{2} / c\right)^{2}$ for $j=1,2,3, z^{2}=\varepsilon_{3}\left(\omega R_{3} / c\right)^{2}$ y $c$ es la velocidad de la luz.

La Figura B.9 muestra la parte imaginaria de la polarizabilidad de NPs de $\mathrm{Fe}_{3} \mathrm{O}_{4}$ con $R=500 \mathrm{~nm}$ y de $\mathrm{NPs}$ de $\mathrm{Fe}_{3} \mathrm{O}_{4}-\mathrm{SiO}_{2}$ - $\mathrm{Au}$ con radio de magnetita $R_{1}=500 \mathrm{~nm}$ y radio de $\mathrm{SiO}_{2}$ de $R_{2}=510 \mathrm{~nm}$ para distintos valores del radio $R_{3}$ de $\mathrm{Au}$. $\mathrm{Al}$ incrementar el espesor de la cubierta de $\mathrm{Au}$, se produce un corrimiento del máximo hacia longitudes 
de onda mas largas sin producirse cambio en la magnitud de este máximo (los valores son por unidad de volumen de la NP). Para NPs de $\mathrm{Fe}_{3} \mathrm{O}_{4}$ sin cubierta $\beta_{5}=0$ y la polarizabilidad se reduce a la ecuación B.77.

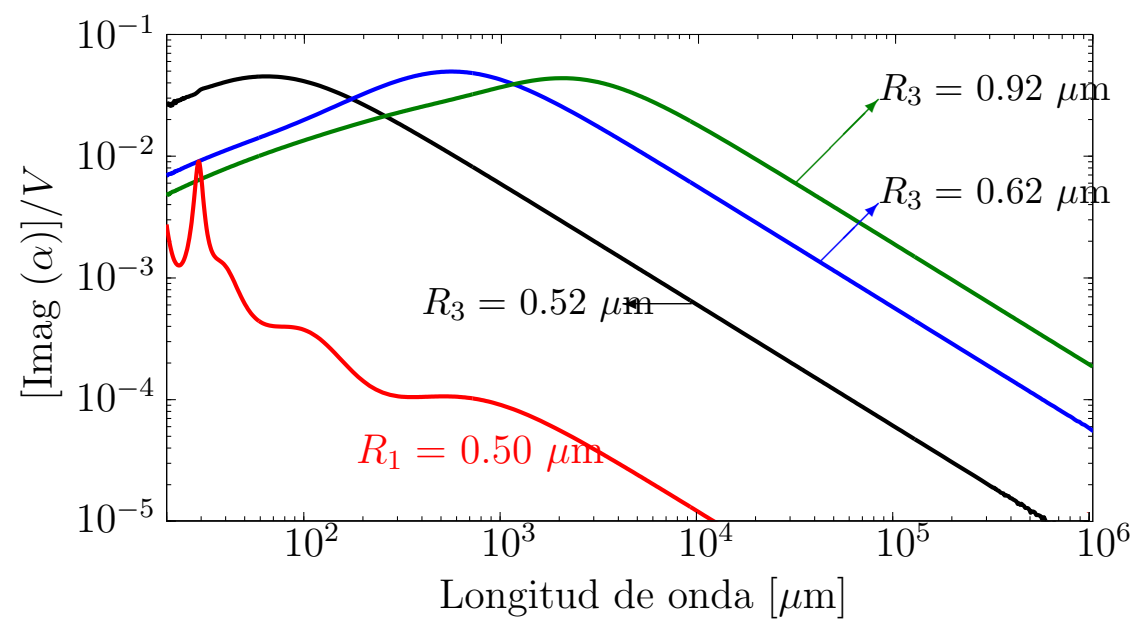

Figura B.9: Gráfica de la parte imaginaria de la polarizabilidad de NPs de con núcleo de Magnetita, primera cubierta de $\mathrm{SiO}_{2}$ y cubierta externa de $\mathrm{Au}$, para diferentes valores de $R_{3}, R_{1}=0.50 \mu \mathrm{m}$ y $R_{2}=0.51 \mu \mathrm{m}$. En rojo solo Magnetita

Como se mostró anteriormente, para NPs de magnetita sin cubierta de radios [20, 500] nm, las corrientes de Eddy son relevantes para longitudes de onda menores de $1 \mu \mathrm{m}$. Este rango de relevancia de las corrientes de Eddy se ve afectado por el espesor de las capas $\mathrm{SiO}_{2}$ y Au, desplazándose a una longitud de onda más larga cuando crece el espesor. 


\section{Apéndice $\mathrm{C}$}

\section{Aproximación de Dipolos Discretos (DDA)}

Cuanto más se dividen los obstáculos son más fáciles de vencer

Concepción Arenal.

Cuando las NEs no son esféricas, obtener soluciones analíticas es muy difícil. En este caso se recurre a métodos numéricos para aproximar las soluciones. Uno de estos métodos consiste en considerar que la NEs se encuentra compuesta por un número $N$ de dipolos, cada uno de ellos de diámetro $d$ (aproximación de dipolos discretos). Para que cada uno de estos dipolos sea considerado como tal en está aproximación, el diámetro cada uno de ellos debe cumplir la condición:

$$
d \leq \frac{1}{|m k|}
$$

donde $m$ es la razón entre el índice de refracción de la estructura y el índice de refracción del medio en el cual se encuentra la estructura y $k$ es el número de onda de la luz incidente. Al incidir el campo eléctrico sobre la estructura cada uno de los dipolos que la conforman adquiere un momento p, con lo cual el campo resultante en la coordenada ocupada por un dipolo es el campo incidente más la suma de los campos producidos por los otros dipolos que conforman la NEs. Para obtener el campo producido por cada uno de los dipolos se conoce que el potencial vectorial producido en un punto de ubicación espacial $\mathbf{r}_{j}$, Figura C.1 por un dipolo de momento dipolar $\mathbf{p}_{i}$ ubicado en $\mathbf{r}_{i}$ es: 


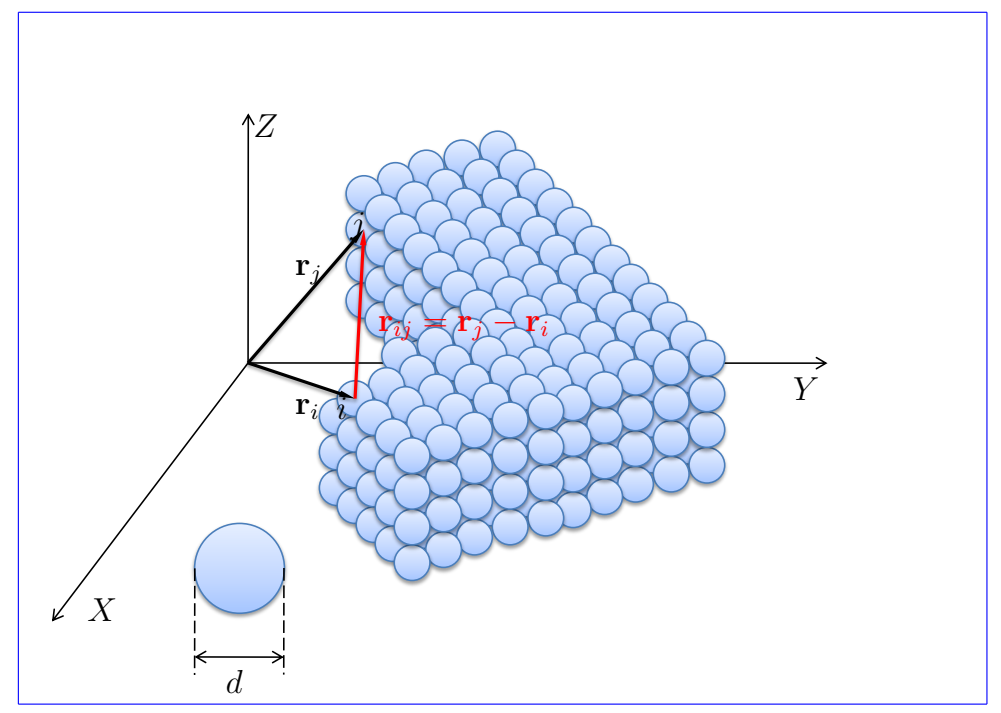

Figura C.1: Geometría para determinar el campo producido por el i-esimo dipolo de una NE compuesta por $N$ dipolos, en la posición del j-esimo dipolo

$$
\mathbf{A}=\frac{\mu_{0}}{4 \pi} \int \mathbf{J}\left(\mathbf{r}^{\prime}\right) d^{3} \mathbf{r}^{\prime}=-\frac{\mu_{0} i \omega}{4 \pi} \frac{e^{i k r}}{r} \mathbf{p}_{i}
$$

donde $r=r_{i j}=\left|\mathbf{r}_{j}-\mathbf{r}_{i}\right|$ y recordando las relaciones entre el potencial vectorial y los campos eléctrico y magnético

$$
\mathbf{H}=\frac{1}{\mu_{0}} \nabla \times \mathbf{A}, \quad \mathbf{E}=\frac{i \sqrt{\mu_{0}}}{k \sqrt{\epsilon_{0}}} \nabla \times \mathbf{H},
$$

la expresión para el campo eléctrico en la posición $\mathbf{r}_{j}$, producido por un dipolo de momento dipolar $\mathbf{p}$ ubicado en la posición $\mathbf{r}_{i}$ se escribe como:

$$
\mathbf{E}_{i j}=\left\{\frac{k^{2}}{r}\left(\mathbf{n} \times \mathbf{p}_{i}\right) \times \mathbf{n}+\left[3 \mathbf{n}\left(\mathbf{n} \cdot \mathbf{p}_{i}\right)-\mathbf{p}_{i}\right]\left(\frac{1}{r^{3}}-\frac{i k}{r^{2}}\right)\right\} e^{i k r}
$$

donde $\mathbf{n}=\mathbf{r}_{i j} / r_{i j}$. El campo eléctrico en la posición $\mathbf{r}_{j}$, se describe como la suma del campo incidente más la contribución de los otros dipolos constituyentes de la estructura.

$$
\mathbf{E}_{j}=\mathbf{E}_{i n c, j}+\sum_{i \neq j}^{N} \mathbf{E}_{i j}
$$

donde el campo eléctrico incidente, se considera una onda plana, que en la posición $\mathbf{r}_{j}$, puede ser escrita como $\mathbf{E}_{i n c, j}=\mathbf{E}_{0} e^{i\left(\mathbf{k} \cdot \mathbf{r}_{j}-\omega t\right)}$ y $\omega$ es la frecuencia de la onda. En 
la expresión para el campo eléctrico producido por un dipolo, se puede factorizar el momento dipolar para escribir el campo eléctrico de forma mas compacta:

$$
\mathbf{E}_{i j}=-\frac{e^{i k r}}{r}\left\{k^{2}(\mathbf{n} \odot \mathbf{n}-I)+\left(\frac{i k r-1}{r^{2}}\right)(3 \mathbf{n} \odot \mathbf{n}-I)\right\} \mathbf{p}=-A_{i j} \mathbf{p}_{i}
$$

donde se se define la matriz $A_{i j}$, para todo $i \neq j$

$$
A_{i j}=\left\{\left(\frac{k^{2}}{r}+\frac{3 i k}{r^{2}}-\frac{3}{r^{3}}\right) \mathbf{n} \odot \mathbf{n}+\left(-\frac{k^{2}}{r}-\frac{i k}{r^{2}}+\frac{1}{r^{3}}\right) I\right\} e^{i k r}
$$

donde la matriz $I$ y el producto $\odot$ se definen para un $\mathbf{n}=\left\langle n_{1}, n_{2}, n_{3}\right\rangle$ como:

$$
I=\left[\begin{array}{ccc}
1 & 0 & 0 \\
0 & 1 & 0 \\
0 & 0 & 1
\end{array}\right], \quad \mathbf{n} \odot \mathbf{n}=\left[\begin{array}{ccc}
n_{1}^{2} & n_{1} n_{2} & n_{1} n_{3} \\
n_{2} n_{1} & n_{2}^{2} & n_{2} n_{3} \\
n_{3} n_{1} & n_{3} n_{2} & n_{3}^{2}
\end{array}\right],
$$

El campo eléctrico en un dipolo de momento dipolar $\mathbf{p}_{j}$, que posee polarizabilidad $\alpha$ es $\mathbf{p}_{j} / \alpha$, con esta definición el conjunto de ecuaciones C.5. se convierte en:

$$
\mathbf{E}_{i n c, j}=\mathbf{p}_{j} / \alpha_{j}-\sum_{i \neq j}^{N} A_{i j} \mathbf{p}_{i}=\sum_{i=1}^{N} A_{i j} \mathbf{p}_{i}
$$

donde $A_{j j}=1 / \alpha_{j}$ y $\alpha_{j}$ es la polarizabilidad del j-esimo dipolo de la NE. La solución del sistema de ecuaciones de $3 N$ incógnitas definido por el sistema C.9, produce las componentes de los $N$ momentos dipolares de la NE considerada. Para NEs esféricas, la polarizabilidad según la expresión de Clausius-Mossotti obtenida en la aproximación dipolar de Mie para NPs de núcleo desnudo se define como:

$$
\alpha_{C M}=a^{3} \frac{\varepsilon-\varepsilon_{m}}{\varepsilon+2 \varepsilon_{m}}
$$

donde $\varepsilon$ es la permitividad de la NP, $\varepsilon_{m}$ es la permitividad del medio en el cual se encuentra la NP y a es el radio de la partícula; usualmente los dipolos que se toman en una estructura definida, son cubos de lado $l$, en cuyo caso el volumen se considera como el de una esfera de radio efectivo definido por $a_{e f}=\sqrt{\frac{3}{4 \pi}} l$. 


\section{Apéndice D Hibridación}

Poesia es la union de dos palabras que uno nunca supuso que pudieran juntarse, y que forman algo asi como un misterio.

Federico García Lorca.

La resolución analítica de las ecuaciones de Maxwell, semi-analítica o numérica utilizando métodos tales como la teoría de Mie, el método multipolar, el método de diferencias finitas, el método de los elementos de contorno y la aproximacion de dipolos discretos, proporcionan métodos útiles para la simulación de la propagación de la luz dentro de estas nanoestructuras y modelar sus propiedades ópticas; sin embargo, no dan una completa comprensión física de las interacciones entre los plasmónes y sus influencias en las propiedades ópticas de las nanoestructuras, como si lo permite la teoría de hibridación [172 180].

El principio de hibridación proporciona un concepto muy simple. Está teoría consiste en calcular las frecuencias de resonancias plasmónicas de nanopartículas metálicas complejas, a partir de las interacciones electromagnéticas de plasmónes de nanoestructuras plasmónicas mas simples. El cálculo de estos plasmónes puede ser más sencillo dependiendo de la geometría de la nanopartícula. El problema es esencialmente equivalente a la forma en que los orbitales atómicos interactúan y forman orbitales moleculares en la teoría de la estructura electrónica. 


\section{D.1. Plasmones en esferas metálicas}

Siguiendo el enfoque presentado por Prodan et al. [135], los plasmones se pueden considerar como deformaciones irrotacionales e incompresibles del gas de electrones de conducción de la partícula en consideración. Para simplificar el desarrollo se supone que los electrones de conducción forman un líquido de densidad de electrones uniforme $n_{0}$. Cuando el objetivo es la determinación de las frecuencias plasmónicas, se puede despreciar cualquier amortiguación. Con las condiciones anteriores se pueden modelar los electrones de conducción como un fluido incompresible e irrotacional con un fondo de densidad de carga uniforme $\rho_{0}$ que representa los iones. La densidad de carga de los electrones $\rho=n_{0}$ e y la corriente $\mathbf{j}$ deben satisfacer la ecuación de continuidad e irrotacionalidad:

$$
\frac{\partial \rho}{\partial t}+\nabla \cdot \mathbf{j}=0 \quad \nabla \times \mathbf{j}=0
$$

La corriente es relacionada con el potencial $\Phi$ a través de la ley de $\mathrm{Ohm} \mathbf{j}=$ $\sigma_{c} \nabla \Phi$, donde $\sigma_{c}$ es la conductividad eléctrica. De la ecuación anterior se escribe $\mathbf{j}=n_{0} e \nabla \eta=\rho \mathbf{v}$, donde $\mathbf{v}$ es la velocidad del fluido en una posición determinada $\mathbf{y}$ $\eta=\sigma_{c} \Phi / n_{0}$ e y satisface la ecuación de Laplace $\nabla^{2} \eta=0$. Para pequeñas deformaciones del fluido, una distribución de carga neta igual a $\rho-\rho_{0}$ aparece alrededor de la superficie metálica. En el límite de pequeñas deformaciones la respuesta es lineal, y la distribución de carga neta $\rho-\rho_{0}$, puede ser vista como una densidad superficial de

carga $\sigma=\lim _{\delta S \rightarrow 0} \frac{1}{\delta S} \int_{\delta V}\left(\rho-\rho_{0}\right) d V$, que utilizando la ecuación de continuidad se puede escribir como:

$$
\frac{\partial \sigma}{\partial t}=\mathbf{n} \cdot \mathbf{j}=n_{0} e \frac{\partial \eta}{\partial \mathbf{n}}
$$

donde $\mathbf{n}$ es un vector normal a la superficie. La energía cinética del fluido se describe en términos de la función $\eta$

$$
T=\frac{1}{2} \int n_{0} m_{e}(\mathbf{v})^{2} d V=\frac{1}{2} n_{0} m_{e} \int \eta \nabla \eta \cdot d \mathbf{S}
$$


donde $m_{e}$ es la masa del electrón. La energía potencial del fluido esta dada por

$$
V=\frac{1}{2} \int \frac{\sigma(\mathbf{r}) \sigma\left(\mathbf{r}^{\prime}\right)}{4 \pi \varepsilon_{0}\left|\mathbf{r}-\mathbf{r}^{\prime}\right|} d S d S^{\prime}
$$

que en el caso de considerar el retardo, se añade la dependencia temporal

$$
V=\frac{1}{2} \int \frac{\sigma(\mathbf{r}, t) \sigma\left(\mathbf{r}^{\prime}, t-\left|\mathbf{r}-\mathbf{r}^{\prime}\right| / c\right)}{4 \pi \varepsilon_{0}\left|\mathbf{r}-\mathbf{r}^{\prime}\right|} d S d S^{\prime}
$$

Las soluciones de la ecuación de Laplace en coordenadas esféricas para $\eta$, en el caso de no presentar retardo, son de la forma $r^{l} e^{i m \psi} P_{l}^{m}(\cos \theta)=r^{l} Y_{l m}(\Omega)$ y $r^{-l-1} e^{i m \psi} P_{l}^{m}(\cos \theta)=r^{-l-1} Y_{l m}(\Omega)$. Fuera de una esfera metálica la solución $r^{-l-1} Y_{l m}(\Omega)$, diverge cuando $r \rightarrow \infty$ y la solución para $\eta$ se describe $\eta(t ; r, \Omega)=$ $\sum_{l, m} A_{l m}(t) r^{l} Y_{l m}(\Omega)$, que se puede escribir en forma mas conveniente y normalizada para una esfera de radio $b$ :

$$
\eta(t ; r, \Omega)=\sum_{l, m} \sqrt{\frac{1}{l b^{2 l+1}}} \dot{S}_{l m} r^{l} Y_{l m}(\Omega),
$$

Para calcular la energía cinética, primero debemos determinar la componente en la dirección radial del gradiente de $\eta$, como $\nabla_{r} \eta(t ; r, \Omega)=\sum_{l, m} \sqrt{\frac{l}{b^{2 l+1}}} \dot{S}_{l m} r^{l-1} Y_{l m}(\Omega)$. Tras remplazar el potencial y el gradiente de $\eta$ en $\mathbf{D . 3}$, se obtiene la energía cinetica

$$
T_{e s}=\frac{n_{0} m_{e}}{2} \sum_{l, m} \dot{S}_{l m}^{2}(t)
$$

Utilizando la solución para $\eta$ en la ecuación D.2 y recordando que en este caso $\partial \eta / \partial \mathbf{n}=\partial \eta / \partial r$ obtenemos la densidad superficial de carga en la superficie de la esfera

$$
\sigma_{e s}(t ; \Omega)=n_{0} e \sum_{l, m} \sqrt{\frac{l}{b^{3}}} S_{l m}(t) Y_{l m}(\Omega)
$$

El desarrollo multipolar $\frac{1}{\left|\mathbf{r}-\mathbf{r}^{\prime}\right|}=\sum_{l, m} \frac{4 \pi}{2 l+1} \frac{\left(r^{\prime}\right)^{l}}{r^{l+1}} Y_{l m}^{*}(\Omega) Y_{l m}(\Omega)$, donde en este caso $r^{\prime} \approx r=b$ y la densidad de carga superficial en la expresión D.4 para la energía potencial producen 


$$
V_{e s}=\frac{\omega_{p}^{2} n_{0} m_{e}}{2} \sum_{l m} \frac{l}{2 l+1} S_{l m}^{2}(t)=\frac{n_{0} m_{e}}{2} \sum_{l m} \omega_{e s, l} S_{l m}^{2}(t),
$$

donde $\omega_{e s, l}=\omega_{p} \sqrt{\frac{l}{2 l+1}}$. El lagrangiano del sistema definido como $L_{e s}=T_{e s}-V_{e s}$, se obtiene a partir de las energías como [135, 181]:

$$
L_{e s}=\frac{n_{0} m_{e}}{2} \sum_{l m}\left[\dot{S}_{l m}^{2}-\omega_{e s, l}^{2} S_{l m}^{2}\right]
$$

Las trayectorias que dan la evolución temporal del sistema son curvas diferenciales, que pueden calcularse a partir de las ecuaciones de Euler-Lagrange $\frac{d}{d t}\left(\frac{\partial L}{\partial \dot{S}_{l m}}\right)-\frac{\partial L}{\partial S_{l m}}=$ 0 a saber $\ddot{S}_{l m}-\omega_{e s, l}^{2} S_{l m}=0$, que representa un movimiento oscilatorio de frecuencia $\omega_{e s, l}$.

En este punto, tomamos un camino diferente al enfoque dado por Prodan et al. 135] para determinar la frecuencia de resonancia de una esfera metálica de radio $R_{2}$, resolviendo la ecuación de Laplace para el potencial en cada una de las regiones $r<R_{2}$ y $r>R_{2}$. Las soluciones son de la forma $\Phi_{r<R_{2}}=\sum_{l=0}^{\infty} A_{l} r^{l} P_{l}(\cos \theta)$ y $\Phi_{r>R_{2}}=\sum_{l=0}^{\infty}\left[B_{l} r^{l}+C_{l} r^{-l-1}\right] P_{l}(\cos \theta)$. Del cumplimiento de las condiciones de frontera se pueden expresar las constantes $A_{l}$ y $C_{l}$ en términos de $B_{l}$

$$
\begin{aligned}
A_{l} & =\frac{\varepsilon_{m}(2 l+1)}{l \varepsilon+\varepsilon_{m}(l+1)} B_{l} \\
C_{l} & =\frac{l\left(\varepsilon_{m}-\varepsilon\right)}{l \varepsilon+\varepsilon_{m}(l+1)} B_{l} R_{2}^{2 l+1}
\end{aligned}
$$

Las ecuaciones anteriores implican que la resonancia del sistema ocurre cuando se cumple la condición $F(\omega)=l \varepsilon+\varepsilon_{m}(l+1)=0$, que en el caso de $\varepsilon_{m}=1$ y $\varepsilon=1-\omega_{p}^{2} / \omega^{2}$, se convierte $\omega_{e s, l}$, resultado que concuerda con lo obtenido por Prodan et al.

Como hemos visto en el Capítulo 2, la función dieléctrica puede ser descrita como la suma de las contribuciones de los electrones libre y de los electrones ligados. Algunos autores suponen por simplicidad que la contribución de los electrones ligados es pequeña y si la constante de amortiguamiento $\gamma$ también es pequeña en comparación 
con $\omega\left[\right.$ nordlander], la función dieléctrica se describe por $\varepsilon_{1}(\omega)=1-\frac{\omega_{p}^{2}}{\omega^{2}}$. El caso $\omega>>\gamma$ puede considerarse válido en metales como el $\mathrm{Au}$ y la Ag, pero en otros metales como el Al no se cumple esta condición y la aproximación de la función dieléctrica sería $\varepsilon_{2}(\omega)=1-\frac{\omega_{p}^{2}}{\omega^{2}-i \gamma \omega}$. En esta última definición de la función dieléctrica $\gamma=1 / \tau$, donde $\tau$ es el camino libre medio de los eléctrones. Pero al tener las NPs un tamaño pequeño se modifica el camino libre medio, obteniéndose como función dieléctrica la expresión $\varepsilon_{3}(\omega)=1-\frac{\omega_{p}^{2}}{\omega^{2}-i \omega \gamma-i \omega C v_{F} / R}$. Sin embargo la contribución de los electrones ligados no es pequeña en la región donde ocurren las resonancias plasmónicas y debe ser considerada.

Algunos autores, por ejemplo [maier cuboidales], incluyen una constante para tener en cuenta esta contribución, de modo que la expresión de la función dieléctrica queda como $\varepsilon_{4}(\omega)=\varepsilon_{\infty}-\frac{\omega_{p}^{2}}{\omega^{2}-i \gamma \omega}$. Esta aproximación no tiene en cuenta la dependencia de la contribución de los electrones ligados con la frecuencia. En este trabajo de tesis se avanzó en una descripción general de la función dieléctrica que contuviera la dependencia con la frecuencia de las transiciones interbanda que dan cuenta de la contribución de los electrones ligados, como así también su dependencia con el tamaño. De esta forma, la expresión general toma la forma:

$$
\begin{aligned}
\varepsilon_{5}(\omega, R)= & \varepsilon_{v}(\omega)+\frac{\omega_{p}^{2}}{\omega(\omega+i \gamma)}-\frac{\omega_{p}^{2}}{\omega\left(\omega+i \gamma_{c}(R)\right)} \\
& -e^{-R / R_{0}} K_{b} \int_{\omega_{g}}^{\infty} \frac{\sqrt{x-\omega_{g}}}{x}[1-F(x, T)] \frac{d x}{x^{2}-\omega^{2}+\gamma_{l i g}^{2}-2 i \omega \gamma_{l i g}}
\end{aligned}
$$

La ubicación del pico plasmónico para NPs de $\mathrm{Au}$ de $25 \mathrm{~nm}$ de radio en $\mathrm{SiO}_{2}$ como medio utilizando las funciones dieléctricas descritas por $\varepsilon_{1}, \varepsilon_{2}$ y $\varepsilon_{3}$ es $246 \mathrm{~nm}$ y utilizando $\varepsilon_{4}$ y $\varepsilon_{5}$ es $538 \mathrm{~nm}$. El ancho medio de cada uno de los picos es $0,4,5.5,25.8$ y $56.2 \mathrm{~nm}$ respectivamente.

La Figura D.1, muestra los espectros de extinción calculados de NPs de Au de 25 nm de radio en $\mathrm{SiO}_{2}$, donde se han utilizado las expresiones de la función dieléctrica antes detalladas. En negro utilizando $\varepsilon_{1}$, donde se observa que el ancho del pico es 
despreciable; en rojo se utilizo $\varepsilon_{2}$, con un incremento del ancho medio del pico debido a la consideración de $\tau$; en azul utilizando $\varepsilon_{3}$ con un ancho medio mayor debido a la corrección del camino libre medio; en violeta utilizando $\varepsilon_{4}$, con un valor de $\varepsilon_{\infty}=12.3$ ajustado para que la ubicación del pico plasmónico de una nanopartícula de $25 \mathrm{~nm}$ coincida con la experimental; finalmente, en dorado se muestra el espectro de extinción utilizando $\varepsilon_{5}$.

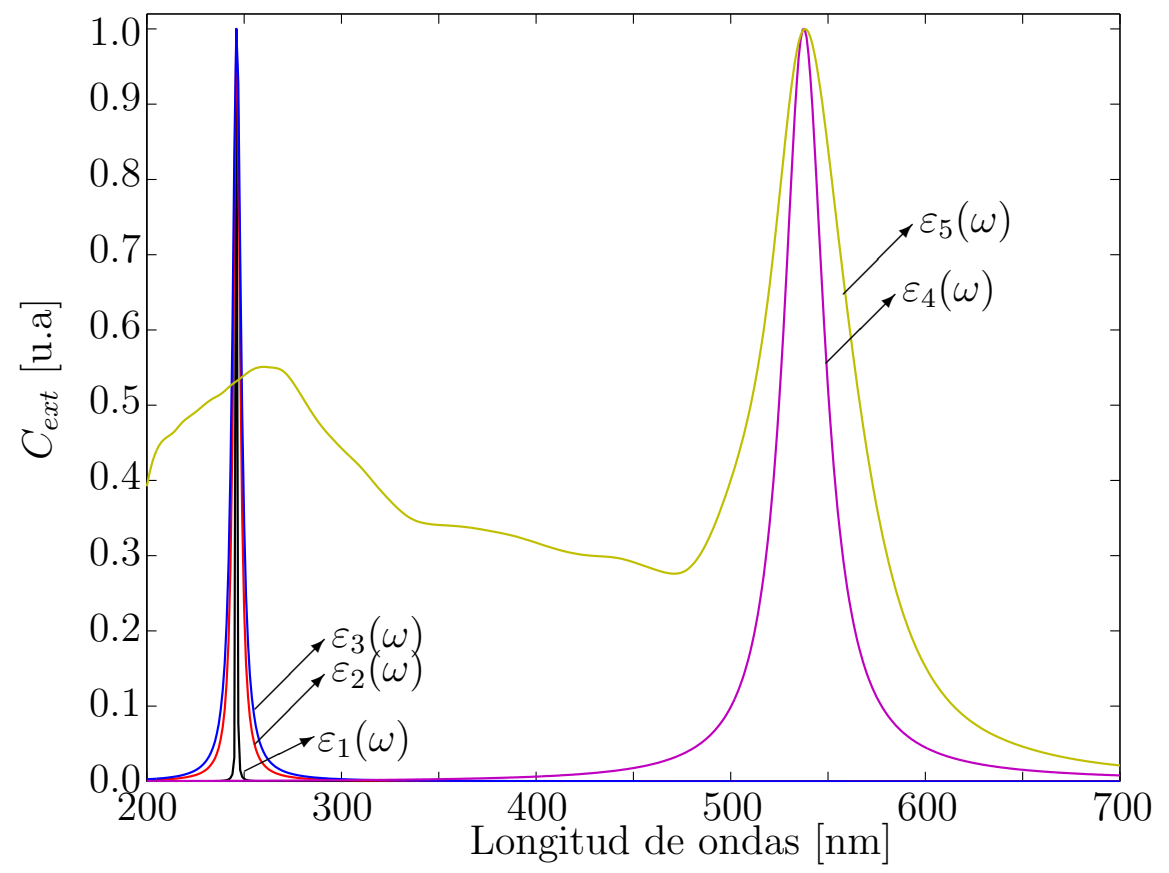

Figura D.1: Espectros de extinción de nanopartículas de Au de $25 \mathrm{~nm}$ de radio en $\mathrm{SiO}_{2}$ como medio, utilizando la aproximación dipolar y distintas aproximaciones de la función dieléctrica.

Puede observarse que esta última función dieléctrica permite reproducir el espectro experimental con mayor fidelidad (como se ilustro en el cap 4) no solamente en la ubicación de la resonancia plasmonica y su ancho medio sino también en los rasgos por debajo de $450 \mathrm{~nm}$ producidos por la absorción de las transiciones interbanda. Es importante observar que el valor utilizado para $\varepsilon_{\infty}$ reproduce la ubicación del pico de extinción solo para el radio escogido. En caso de tener que ajustar un espectro correspondiente a otro radio de $\mathrm{NP}$ el valor de $\varepsilon_{\infty}$ debe cambiar, haciéndolo por tando dependiente del tamaño. 


\section{D.2. Plasmones en cavidades esféricas}

Para una cavidad esférica de radio $R_{1}$, las soluciones $r^{l} Y_{l m}(\Omega)$ divergen cuando $r \rightarrow 0$ y por tanto las soluciones para $\eta$ normalizadas se describen

$$
\eta(t ; r, \Omega)=\sum_{l, m} \sqrt{\frac{R_{1}^{2 l+1}}{l+1}} \dot{C}_{l m} r^{-l-1} Y_{l m}(\Omega)
$$

Si aplicamos el mismo procedimiento que en la sección anterior para determinar el Lagrangiano para la cavidad esférica metálica $[\mathbf{1 3 5}, \mathbf{1 8 1}$

$$
L_{c a}=\frac{1}{2} n_{0} m_{e} \sum_{l m}\left[\dot{C}_{l m}^{2}-\omega_{c a, l}^{2} C_{l m}\right],
$$

donde $\omega_{c a, l}=\omega_{p} \sqrt{\frac{l+1}{2 l+1}}$, representa la frecuencia plasmónica de la cavidad esférica metálica, que en el caso de considerar el retardo esta frecuencia plasmónica es $\omega_{c a, l}^{r e t}=\omega_{c a, l} R_{l m}$. Si se realiza la solución de la ecuación de Laplace para el caso de una cavidad esferica la condición de resonancia ocurre cuando $l \varepsilon_{m}+\varepsilon(l+1)=0$.

\section{D.3. Plasmones en nano-cubiertas metálicas}

La geometría considerada es una nano-cubierta de radio interno $R_{1}$ y radio externo $R_{2}$. Para esta geometría la solución de la función $\eta$ normalizada es de la forma

$$
\eta(t ; r, \Omega)=\sum_{l, m} \sqrt{\frac{R_{1}^{2 l+1}}{l+1}} \dot{C}_{l m} r^{-l-1} Y_{l m}(\Omega)+\sqrt{\frac{1}{l R_{2}^{2 l+1}}} \dot{S}_{l m} r^{l} Y_{l m}(\Omega)
$$

En las superficies interior y exterior aparecen densidades superficiales de carga $\sigma_{i n}=n_{0} e \nabla_{r} \eta\left(t ; R_{1}, \Omega\right)$ y $\sigma_{e x}=n_{0} e \nabla_{r} \eta\left(t ; R_{2}, \Omega\right)$, que apartir de $\eta$, se calculan como:

$$
\begin{aligned}
\sigma_{i n}(t ; \Omega) & =n_{0} e \sum_{l m}\left(\sqrt{\frac{l+1}{R_{1}^{3}}} C_{l m}(t)-x^{l-1} \sqrt{\frac{l}{R_{2}^{3}}} S_{l m}(t)\right) Y_{l m}(\Omega), \\
\sigma_{e x}(t ; \Omega) & =n_{0} e \sum_{l m}\left(-x^{l+2} \sqrt{\frac{l+1}{R_{1}^{3}}} C_{l m}(t)+\sqrt{\frac{l}{R_{2}^{3}}} S_{l m}(t)\right) Y_{l m}(\Omega),
\end{aligned}
$$


donde $x=R_{1} / R_{2}$. Recordando que la energía potencial se escribe como

$$
\begin{aligned}
V & =\frac{1}{2} \sum_{l, m} \int \sigma(\mathbf{r})\left[\frac{4 \pi}{2 l+1} Y_{l m}(\Omega) \cdot \int \frac{\left(r^{\prime}\right)^{l}}{r^{l+1}} \sigma\left(\mathbf{r}^{\prime}\right) Y_{l m}\left(\Omega^{\prime}\right) d S^{\prime}\right] d S \\
& =\frac{n_{0} m_{e}}{2} \sum_{l m}\left(1-x^{2 l+1}\right)\left[\omega_{c a, l}^{2} C_{l m}^{2}-2 \omega_{c a, l} \omega_{e s, l} x^{l+1 / 2} C_{l m} S_{l m}+\omega_{e s, l}^{2} S_{l m}^{2}\right](D)
\end{aligned}
$$

La energía cinetica de la cubierta se determina a partir de la función $\eta$ como:

$$
\begin{aligned}
T & =\frac{n_{0} m_{e}}{2} \int\left[\left.\eta \nabla_{r} \eta \cdot R_{2}^{2}\right|_{r=R_{2}}-\left.\eta \nabla_{r} \eta \cdot R_{1}^{2}\right|_{r=R_{1}}\right] d \Omega \\
& =\frac{n_{0} m_{e}}{2} \sum_{l m}\left(1-x^{2 l+1}\right)\left(\dot{S}_{l m}^{2}+\dot{C}_{l m}^{2}\right)
\end{aligned}
$$

Utilizando las energías cinética y potencial, las ecuaciones de Euler-Lagrange para una cubierta metálica se describen por el Lagrangiano [135, 181

$$
L_{n c}=\frac{n_{0} m_{e}}{2} \sum_{l m}\left(1-x^{2 l+1}\right)\left[\dot{S}_{l m}^{2}+\dot{C}_{l m}^{2}-\omega_{c a, l}^{2} C_{l m}^{2}-2 \omega_{c a, l} \omega_{e s, l} C_{l m} S_{l m} x^{1+1 / 2}+\omega_{e s, l}^{2} S_{l m}^{2}\right]
$$

y produce el sistema de ecuaciones diferenciales para $S_{l m}$ y $C_{l m}$, sistema que presenta soluciones que se pueden describir en la forma $S_{l m}=S e^{i \omega t}$ y $C_{l m}=C e^{i \omega t}$; soluciones que al ser remplazadas en el sistema de ecuaciones diferenciales, produce el sistema de ecuaciones para las constantes $C$ y $S$. Este sistema tiene solución solo cuando el determinante formado por los coeficientes de $S$ y $C$ es igual a cero, en cuyo caso los valores de $\omega$, que representan las soluciones son $[130-132,135-137,180-182]:$

$$
\omega_{l \pm}^{2}=\frac{\omega_{e s, l}^{2}+\omega_{c a, l}^{2} \pm \sqrt{\left(\omega_{e s, l}^{2}+\omega_{c a, l}^{2}\right)^{2}+4 \omega_{e s, l}^{2} \omega_{c a, l}^{2}\left(x^{2 l+1}-1\right)}}{2}
$$


En la Figura D.2 se muestran las frecuencias de resonancia para $l=1$ para diferentes metales $\mathrm{Au}, \mathrm{Ag}, \mathrm{Al}$ y Fe a la izquierda para esferas de radios $R_{2}$ a la derecha para cavidades de radio $R_{1}$ y en la parte central el resultado de la hibridación de los plasmones de la esfera y la cavidad, donde se utiliza la aproximación para la función dieléctrica del metal $\varepsilon(\omega)=1-\omega_{p}^{2} / \omega^{2}$ y los valores de $\omega_{ \pm}$se determinaron utilizando la ecuación D.23.

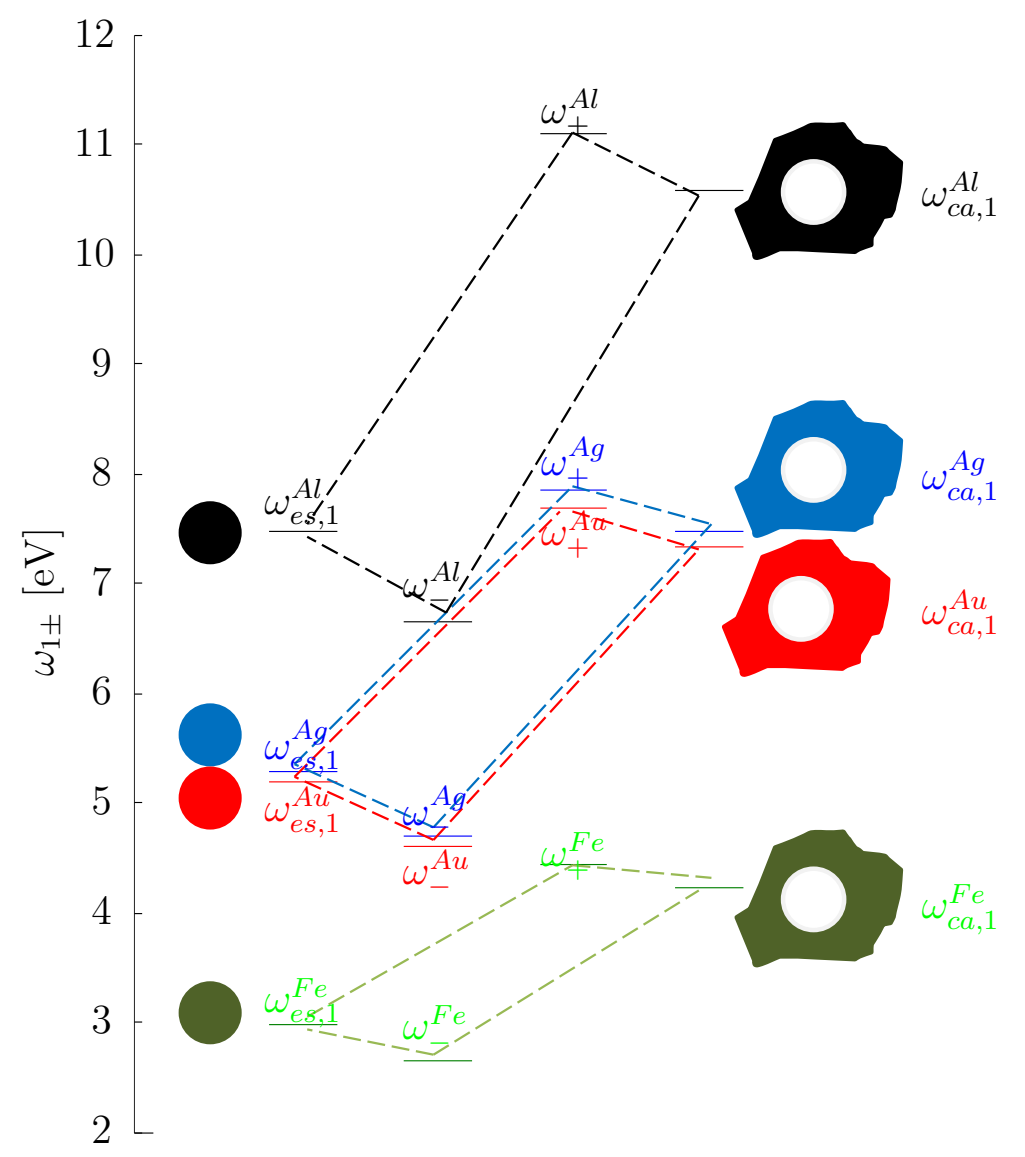

Figura D.2: Frecuencias de resonancia de cavidades, esferas y nanocubiertas de Au, Ag, Al e Fe para $x=R_{1} / R_{2}=20 \mathrm{~nm} / 40 \mathrm{~nm}$

La evolución de las resonancias $\omega_{ \pm}$en una nanocubierta de proporción $x=R_{1} / R_{2}$, se muestra en la Figura D.3, donde en línea continua se muestra la evolución de $\omega_{-}$ y en segmentos cortos la evolución de $\omega_{+}$. Al incrementar el valor de $x$, el valor de la frecuencia de resonancia $\omega_{-}$cae a cero por la disminución del espesor de la nanocubierta, en contraposición la resonancia $\omega_{+}$incrementa su valor. 


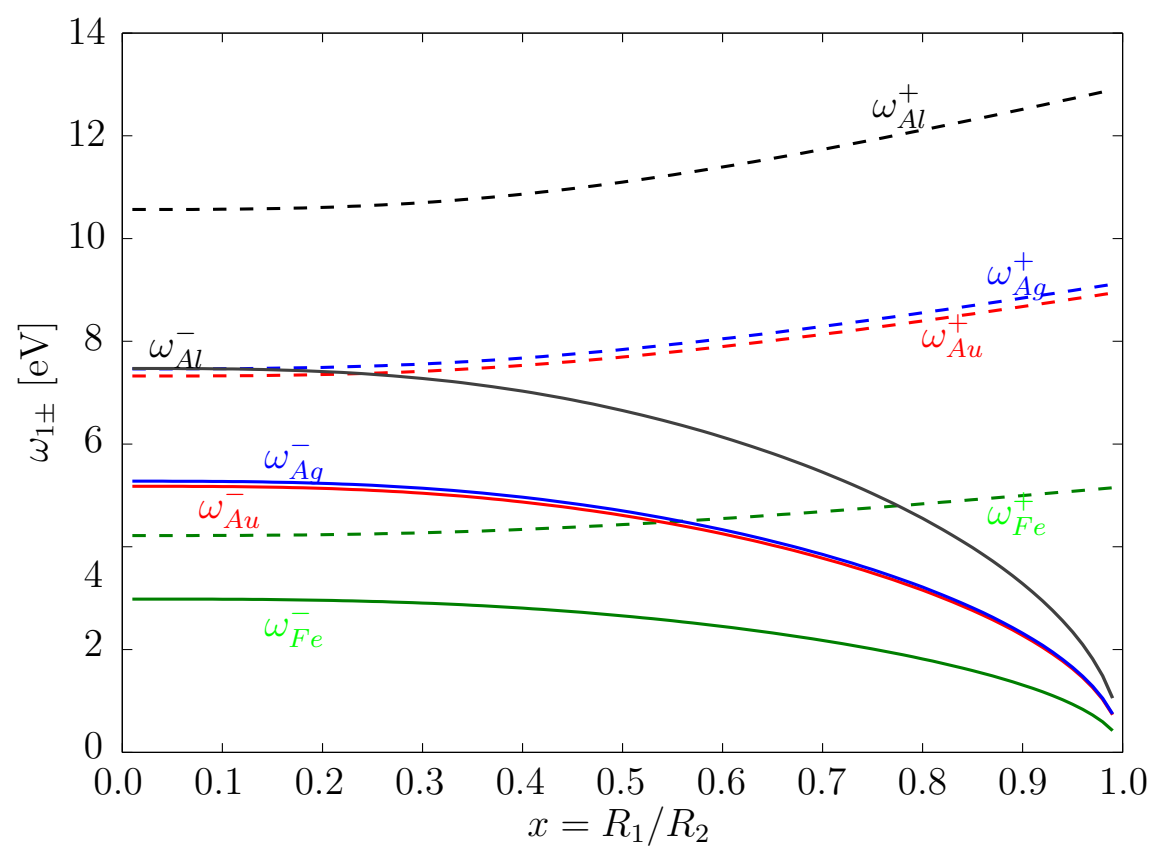

Figura D.3: Frecuencias de resonancia de nanocubiertas de $\mathrm{Au}, \mathrm{Ag}, \mathrm{Al}$ e $\mathrm{Fe}$ para diferentes relaciones $x=R_{1} / R_{2}$

Al solucionar la ecuación de Laplace para una nanocubierta de permitividad $\varepsilon$, con medio interno de permitividad $\varepsilon_{1}$ y medio circundante de permitividad $\varepsilon_{2}$ e imponer las condiciones de frontera adecuadas, la condición de resonancia se obtiene como: $F(\omega)=\varepsilon^{2} l(l+1)\left(x^{-2 l-1}-1\right)+\varepsilon\left[l(l+1)\left(\varepsilon_{1}+\varepsilon_{2}\right)+\left(\varepsilon_{1} l^{2}+\varepsilon_{2}(l+1)^{2}\right) x^{-2 l-1}\right]+$ $\varepsilon_{1} \varepsilon_{2} l(l+1)\left(x^{-2 l-1}-1\right)=0$.

En la Figura D.4, se muestran espectros de extinción para una nanocubierta de $\mathrm{Au}$ en $\mathrm{SiO}_{2}$ con núcleo de $\mathrm{SiO}_{2}$, utilizando las aproximaciones de la función dieléctrica antes mencionadas. Los espectros de extinción obtenidos muestran un comportamiento similar al ilustrado en la Figura D.1. 


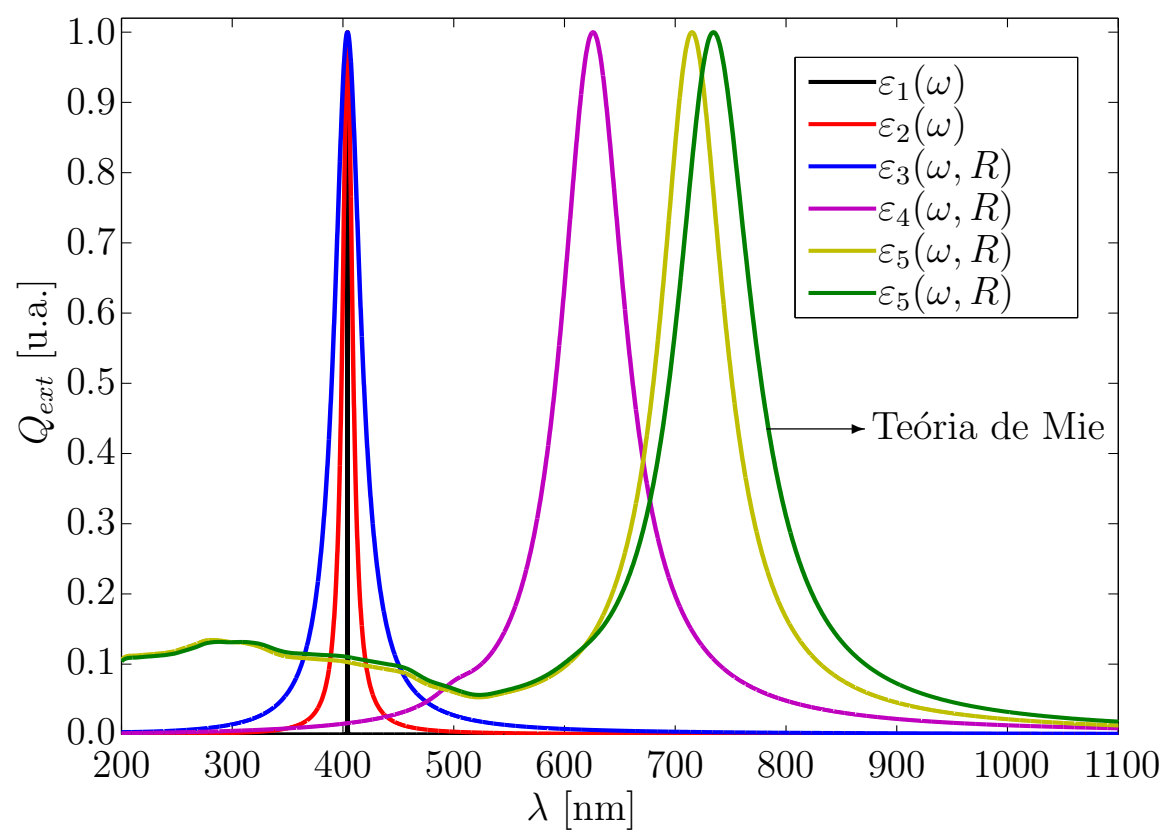

Figura D.4: Extinción de nanocubiertas (20nm-25nm) de Au con núcleo y medio circundante de $\mathrm{SiO}_{2}$ para diferentes aproximaciónes de la función dieléctrica utilizando la aproximación dipolar y en línea verde utilizando la teória de Mie. 


\section{Bibliografía}

[1] Gustav Mie. Beitrage zur optik truber medien, speziell kolloidaler metallosungen. Ann.Phys (Leipzig), 330:377-445, 1908.

[2] Nanophotonics. Wiley-Interscience Publication, 2004.

[3] Principles of Nano-Optics. Cambridge University Press, 2006.

[4] Stefan A. Maier. Plasmonics: Fundamentals and aplications. Springer, 1 edition, 2007.

[5] R.W. Wood. On a remarkable case of uneven distribution of light in a diffraction grating spectrum. Philosophical Magazine Series 6, 4:396-402, 1902.

[6] Heinz Raether. Surface Plasmons: on Smooth and Rough Surfaces and on Gratings. Springer-Verlag, 1 edition, 1986.

[7] J. C. Maxwell Garnett. Colours in metal glasses and in metallic films. Philosophical Transactions of the Royal Society of London. Series A, Containing Papers of a Mathematical or Physical Character, 203:359-371, 1904.

[8] David Pines. Collective energy losses in solids. Rev. Mod. Phys., 28:184, 1956.

[9] Ritchie R H. Plasma losses by fast electrons in thin films. Phys. Rev, 106:874-881, 1957.

[10] U.Kreibig and P. Zacharias. Surface plasma resonances in small spherical silver and gold particles. Z. Physik, 231:128-143, 1970. 
[11] John Davis Jackson. Classical Electrodinamics. John wiley \& sons, 1 edition, 1962.

[12] John R. Reitz, Frederick J. Milford, and Robert W. Christy. Fundamentos de la teoría electromagnética. Adison wesley iberoamericana, 4 edition, 1996.

[13] C. F. Bohren and D. R. Huffman. Absorption and Scattering of Light by Small Particles. John Wiley \& Sons, 1 edition, 1998.

[14] F. J. García de Abajo. Colloquium: Light scattering by particle and hole arrays. Reviews of Modern Physics, 79:1267-1290, 2007.

[15] Colleen L. Nehl, Nathaniel K. Grady, Glenn P. Goodrich, Felicia Tam, Naomi J. Halas, and Jason H. Hafner. Scattering spectra of single gold nanoshells. Nano Letters, 4:2355-2359, 2004.

[16] T. Klar, M. Perner, S. Grosse, G. von Plessen, W. Spirkl, and J. Feldmann. Surface-plasmon resonances in single metallic nanoparticles. Optics Communications, 80:4249-4252, 1998.

[17] Mostafa A. El-Sayed Xiaohua Huang and. Gold nanoparticles: Optical properties and implementations in cancer diagnosis and photothermal therapy. Journal of Advanced Research, 1:13-28, 2010.

[18] S.Link and M. El-Sayed. Spectral properties and relaxation dynamics of surface plasmon electronic oscillations in gold and silver nanodots and nanorods. J. Phys. Chem B, 103:8410, 1999.

[19] J. B. Jackson and N. J. Halas. Silver nanoshells:â variations in morphologies and optical properties. J. Phys. Chem B, 105:2743, 2001.

[20] K. Nelly, E. Coronado, L. Zhao, and G. Schatz. The optical properties of metal nanoparticles: The influence of size, shape, and dielectric environment. J. Phys. Chem B, 107:668, 2003. 
[21] A. D. Rakic, A. B. Djurisic, J. M. Elazar, and M. L. Majewski. Optical properties of metallic films for vertical-cavity optoelectronic devices. Applied Optics, 37:5271-5283, 1998.

[22] Kaminskiene, I. Prosylevasa, J. Stonkute, and A. Guobiene. Evaluation of optical properties of ag, cu, and co nanoparticles synthesized in organic medium. Journal of Chemical Technology and Biotechnology, 123:111-114, 2013.

[23] H. Ehrenreich and H. R. Philipp. Optical properties of Ag and Cu. Physical Review, 128:1622-1629, 1962.

[24] B. R. Cooper and H. enrenreich. Optical properties of noble metals. ii. Physical Review, 138:A494-A507, 1965.

[25] S. Norrman, T. Andersson, C. G. Granqvist, and O. Hunderi. Optical absorption in discontinuous gold films. Solid State Communications, 23:261-265, 1977.

[26] V. A. Markel and Vladimir M. Shalaev. Small-particle composites. i. linear optical properties. Physical Review B, 53:2425-2436, 1996.

[27] T. Klar, M. Perner, S. Grosse, G. von Plessen, W. Spirkl, and J. Feldmann. Surface-plasmon resonances in single metallic nanoparticles. Physical Review Letters, 80:4249-4252, 1998.

[28] J. Perenboom, P. Wyder, and F. Meier. Electronic properties of small metallic particles. Physics Reports(Review Section of Physics Letters), 78:173-292, 1981.

[29] M. Fox. Optical Properties of Solids. Oxford University Press, 1 edition, 2011.

[30] Michael Quinten. Optical Properties of Nanoparticle Systems Mie and Beyond. Wiley VCH, 2 edition, 2011.

[31] Henry Ehrenreich and Frans Spaepen. Solid State Physics. Academic Press, 1 edition, 1997. 
[32] Anatoliy Pinchuk, Gero von Plessen, and Uwe Kreibig. Influence of interband electronic transitions on the optical absorption in metallic nanoparticles. J. Phys. D: Appl. Phys., 37:3133-3139, 2004.

[33] Enrico Massa, Stefan A Maier, and Vincenzo Giannini. An analytical approach to light scattering from small cubic and rectangular cuboidal nanoantennas. New Journal of Physics, 15:1-13, 2013.

[34] Inouye Hideyuki, Tanaka Koichiro, Tanahashi Ichiro, and Hirao Kazuyuki. Ultrafast dynamics of nonequilibrium electrons in a gold nanoparticle system. Physical Review B, 57:11334-11340, May 1998.

[35] J. M. J. Santillán, F. A. Videla, M. B. Fernández van Raap, D. C. Schinca, and L. B. Scaffardi. Size dependent cu dielectric function for plasmon spectroscopy: Characterization of colloidal suspension generated by fs laser ablation. Journal of Applied Physics, 112:319-327, 2012.

[36] J. Bigot, J. Merle, O. Cregut, and A. Daunois. Electron dinamics in copper metallic nanoparticles probed with femtosecond optical pulses. Physical Review Letters, 75:4702-4705, 1995.

[37] Gabriele Giuliani and Giovanni Vignale. Quantum theory of the electron liquid. J Cambridge, 1 edition, 2005.

[38] P. B. Johnson and R. W. Christy. Optical constants of the noble metals. Physical Review B, 6:4370-4379, 1972.

[39] S. Babar and J. H. Weaver. Optical constants of $\mathrm{Cu}, \mathrm{Ag}$, and Au revisited. Applied Optics, 54:477-481, 2015.

[40] K. M. McPeak, S. V. Jayanti, S. J. P. Kress, S. Meyer, S. Iotti, A. Rossinelli, and D. J. Norris. Plasmonic films can easily be better: Rules and recipes. ACS Photonics, 2:326-333, 2015. 
[41] H. J. Hagemann, W. Gudat, and C. Kunz. Optical constants from the far infrared to the x-ray region: $\mathrm{Mg}, \mathrm{Al}, \mathrm{Cu}, \mathrm{Ag}, \mathrm{Au}, \mathrm{Bi}, \mathrm{C}$, and $\mathrm{Al}_{2} \mathrm{O}_{3}$. Journal of the Optical Society of America, 65:742-744, 1974.

[42] Mark A. Ordal, Robert J. Bell, Ralph W. Alexander Jr., Larry L. Long, and Marvin R. Querry. Optical properties of $\mathrm{Au}, \mathrm{Ni}$, and $\mathrm{Pb}$ at submillimeter wavelengths. Applied Optics, 26:744-752, 1987.

[43] D. L. Windt, W. C. Cash, M. Scott, P. Arendt, B. Newnam, R. F. Fisher, and A. B. Swartzlander. Optical constants for thin films of $\mathrm{Ti}, \mathrm{Zr}, \mathrm{Nb}, \mathrm{Mo}, \mathrm{Ru}, \mathrm{Rh}$, Pd, Ag, Hf, Ta, W, Re, Ir, Os, Pt, and Au from 24 a to 1216 a. Applied Optics, $27: 246-278,1988$.

[44] R. L. Olmon, B. Slovick, T. W. Johnson, D. Shelton, S. H. Oh, G. D. Boreman, and M. B. Raschke. Optical dielectric function of gold. Physical Review B, $86: 235147,2012$.

[45] F. Lemarchand L. Gao and M. Lequime. Comparison of different dispersion models for single layer optical thin film index determination. Thin Solid Films, 520:501-509, 2013.

[46] M. A. Ordal, R. J. Bell, R. W. Alexander, L. L. Long, and M. R. Querry. Optical properties of fourteen metals in the infrared and far infrared: $\mathrm{Al}, \mathrm{Co}, \mathrm{Cu}, \mathrm{Au}, \mathrm{Fe}$, Pb, Mo, Ni, Pd, Pt, Ag, Ti, V, and W. Applied Optics, 24:4493-4499, 1985.

[47] K. Stahrenberg, Th. Herrmann, K. Wilmers, N. Esser, W. Richter, and M. J. G. Lee. Optical properties of copper and silver in the energy range 2.5-9.0 ev. Physical Review B, 64:4493-4499, 2001.

[48] N. Brimhall, N. Herrick, D. D. Allred, R. S. Turley, M. Ware, and J. Peatross. Measured optical constants of copper from $10 \mathrm{~nm}$ to $35 \mathrm{~nm}$. Optics Express, 17:23873-23879, 2009. 
[49] P. B. Johnson and R. W. Christy. Optical constants of transition metals: Ti, V, Cr, Mn, Fe, Co, Ni and Pfsd. Physical Review B, 9:5056-5065, 1974.

[50] Vladimir M. Shalaev, E. Y. Poliakov, and V. A. Markel. Small-particle composites. ii. nonlinear optical properties. Physical Review B, 53:2437-2449, 1996.

[51] George Y. Panasyuk, John C. Schotland, and Vadim A. Markel. Classical theory of optical nonlinearity in conducting nanoparticles. Physical Review Letters, 100:047402-1-047402-4, 2008.

[52] Mark A. Ordal, Robert J. Bell, Ralph W. Jr Alexander, Lawrence A. Newquist, , and Marvin R. Querry. Optical properties of Al, Fe, Ti, Ta, W, and Mo at submillimeter wavelengths. Applied Optics, 27:1203-1209, 1988.

[53] Luis J. Mendoza Herrera, David Mu netón Arboleda, Daniel C. Schinca, and Lucía B. Scaffardi. Determination of plasma frequency, damping constant, and size distribution from the complex dielectric function of noble metal nanoparticles. Journal of Applied Physics, 116:233105, 2014.

[54] Luis J. Mendoza Herrera, Lucía B. Scaffardi, and Daniel C. Schinca. High spectral field enhancement and tunability in core-double shell metal-dielectric-metal spherical nanoparticles. RSC Advances, 6:110471, 2016.

[55] L. Smrcka Czech. Energy band structure of aluminium by the augmented plane wave method. Journal of Physics B: Atomic, Molecular and Optical Physics, 20:291-300, 1970.

[56] Harry J. Levinson, F. Greuter, and E. W. Plummer. Experimental band structure of aluminum. Physical Review B, 27:727-747, 1983.

[57] L. B. Scaffardi and J. O. Tocho. Size dependence of refractive index of gold nanoparticles. Nanotechnology, 17:1309-1315, 2006. 
[58] J. M. J. Santillán, F. A. Videla, M. B. Fernández van Raap, L. B. Scaffardi, and D. C. Schinca. Influence of size-corrected bound-electron contribution on nanometric silver dielectric function. sizing through optical extinction spectroscopy. Journal of Physics D: Applied Physics, 46:301-311, 2013.

[59] J. M. J. Santillán, F. A. Videla, M. B. Fernández van Raap, D. C. Schinca, and L. B. Scaffardi. Analysis of the structure, configuration and sizing of $\mathrm{Cu}$ and $\mathrm{Cu}$ oxide nanoparticles generated by fs laser ablation of solid target in liquids. Journal of Applied Physics, 113:305-314, 2013.

[60] H. Hovel, S. Fritz, U. Kreibig, and M. Vollmer. Width of cluster plasmon resonances: Bulk dielectric functions and chemical interface damping. Physical Review B, 48:24-38, 1993.

[61] K. Kolwas and A. Derkachova. Damping rates of surface plasmons for particles of size from nano- to micrometers; reduction of the nonradiative decay. Journal of Quantitative Spectroscopy \&3 Radiative Transfer, 114:45-55, 2013.

[62] U. Kreibig, G. Bour, A. Hilger, and M. Gartz. Optical properties of cluster-matter: Influences of interfaces. physica status solidi (a), 175:351-366, 1999.

[63] U. Kreibig. Electronic properties of small silver particles: the optical constants and their temperature dependence. Journal of Physics F: Metal Physics, 4:999-1014, 1974.

[64] U. Kreibig and C. V. Fragstein. The limitation of electron mean free path in small silver particles. Zeitschrift für Physik, 224:307-323, 1969.

[65] Marcos M. Alvarez, Joseph T. Khoury, T. Gregory Schaaff, Marat N. Shafigullin, Igor Vezmar, , and Robert L. Whetten. Optical absorption spectra of nanocrystal gold molecules. The Journal of Physical Chemistry B, 101:3706-3712, 1997.

[66] D. O. Smith. Magnetization of a Magnetite Single Crystal Near the Curie Point. Phys. Rev., 102(4):959-963, 1956. 
[67] S. Chikazumi. Physics of magnetism. John Wiley \& Sons, New York, 1 edition, 1964.

[68] B. T. Draine and Brandon Hensley. Magnetic nanoparticles in the interstellar medium: Emission spectrum and polarization. The Astrophysical Journal, 765:159-181, 2013.

[69] Ing Kong, Sahrim Hj Ahmad, Mustaffa Hj Abdullah, David Hui, Ahmad Nazlim Yusoff, and Dwi Puryanti. Magnetic and microwave absorbing properties of magnetiteâthermoplastic natural rubber nanocomposites. Journal of Magnetism and Magnetic Materials, 322(21):3401-3409, 2010.

[70] D. Rousselle, A. Berthault, O. Acher, J. P. Bouchaud, and P. G. Zérah. Magnetic and microwave absorbing properties of magnetiteâthermoplastic natural rubber nanocomposites. J. Appl. Phys., 74(1):475, 1993.

[71] Yong-Hui D., Chang-Chun W., Jian-Hua H., Wu-Li Y., and Shou-Kuan F. Investigation of formation of silica-coated magnetite nanoparticles via sol-gel approach. Colloids and Surfaces A: Physicochemical and Engineering Aspects, 262:87-93, 2005.

[72] N Cabrera and N F Mott. Theory of the oxidation of metals. Reports on Progress in Physics, 12(1):163-166, 1949.

[73] G. Litrico, P. Proulx, J.B. Gouriet, and P. Rambaud. Controlled oxidation of aluminum nanoparticles. Advanced Powder Technology, 26(1):1-7, 2015.

[74] N. A. Fuchs. On the stationary charge distribution on aerosol particles in a bipolar ionic atmosphere. Geofisica pura e applicata, 56(1):185-193, 1963.

[75] Vincenzo Amendola. Laser ablation synthesis in solution (lasis) of functional nanoparticles. La Química y la Industria, 1:156-163, 2010.

[76] G.W. Yang. Laser ablation in liquids: Applications in the synthesis of nanocrystals. Progress in Materials Science, 52:648-698, 2007. 
[77] Leonid V. Zhigilei, Zhibin Lin, and Dmitriy S. Ivanov. Atomistic modeling of short pulse laser ablation of metals: Connections between melting, spallation, and phase explosion. J. Phys. Chem. C, 113:11892-11906, 2009.

[78] Leonid V. Zhigilei, Prasad B. S. Kodali, and Barbara J. Garrison. A microscopic view of laser ablation. J. Phys. Chem. C, 102:2845-2853, 1998.

[79] S. Amoruso, M. Armenante, V. Berardi, R. Bruzzese, and N. Spinelli. Absorption and saturation mechanisms in aluminium laser ablated plasmas. Physical Review A, 65:265-271, 1997.

[80] S. Amoruso, R. Bruzzese, M. Vitiello, N. N. Nedialkov, and P. A. Atanasov. Experimental and theoretical investigations of femtosecond of aluminum in vacuum. Journal of Applied Physics, 98:0449071-0449077, 2005.

[81] Umberto Perini Sergio Musazzi, editor. Laser-Induced breakdown spectroscopy: Cap 6. Femtosecond Laser Ablation: Fundamentals and Applications. Springer, 2014.

[82] Donna Strickland and Gerard Mourou. Compression of amplified chirped optical pulses. Optics Communications, 56:219-221, 1985.

[83] Keyffer J. Salas P., Víctor J. Garica, Freddy Fernández-Rojas, and Carlos Fernández-Rojas. Regímenes de ablación láser en la elaboración de películas delgadas. Revista de la Facultad de Ingeniería U.C.V., 25:121-126, 2010.

[84] J. P. Colombier, P. Combis, F. Bonneau, R. Le Harzic, and E. Audouard. Hydrodynamic simulations of metal ablation by femtosecond laser irradiation. Physical Review B, 71:1654061-1654066, 2005.

[85] I. M. Lifshits, M. I. Kaganov, and L. V. Tanatarov. On the theory of radiation-induced changes in metals. Journal of Nuclear Energy Science $\mathcal{E}$ Power Generation, 12:69-78, 1960. 
[86] E. G. Gamaly, A. V. Rode, V. T. Tikhonchuk, and B. Luther-Davies. Ablation of solids by femtosecond lasers: ablation mechanism and ablation thresholds for metals and dielectrics. Physical Review A, 1:1-27, 2001.

[87] J Dieleman, E. Van De Reit, and J.C.S Kools. Laser ablation deposition: Mechanism and application. Journal of Applied Physics, 31:1964-1971, 1992.

[88] S. I. Anisimov, B. L. Kapeliovich, and T. L. Perelman. Electron emission from metal surfaces exposed to ultrashort laser pulses. Soviet Physics JETP, 9:373-375, 1974.

[89] Nan Zhang, Xiaonong Zhu, Jianjun Yang, Xiaolei Wang, and Mingwei Wang. Time-resolved shadowgraphs of material ejection in intense femtosecond laser ablation of aluminum. Physical Review Applied, 99:167602-1-4, 2007.

[90] B. Le Drogoff, F. Vidal, Y. von Kaenel, M. Chaker, T. W. Johnston, and S. Laville. Ablation of aluminum thin films by ultrashort laser pulses. Journal of Applied Physics, 89:8247-8252, 2001.

[91] Katsuya Oguri, Yasuaki Okano, Tadashi Nishikawa, and Hidetoshi Nakano. Dynamical study of femtosecond-laser-ablated liquid-aluminum nanoparticles using spatiotemporally resolved $\mathrm{x}$-ray-absorption fine-structure spectroscopy. Physical Review Letters, 99:165003-1-4, 2007.

[92] E. G. Gamaly, A. V. Rode, and B. Luther-Davies. Ablation of solids by femtosecond lasers: Ablation mechanism and ablation thresholds for metals and dielectrics. Physics of Plasmas, 9:949-957, 2002.

[93] M.I. Kaganov, I. M. Lifshitz, and L. V. Tanatarov. Relaxation between t:lectrons and the crystalline lattice. Soviet Physics JETP, 4:173-178, 1957.

[94] Xu Z., Hou Y., and Sun S. Magnetic core/shell $\mathrm{Fe}_{3} \mathrm{O}_{4} / \mathrm{Au}$ and $\mathrm{Fe}_{3} \mathrm{O}_{4} / \mathrm{Au} / \mathrm{Ag}$ nanoparticles with tunable plasmonic properties. Journal of the American Chemical Society, 129:8698-8699, 2007. 
[95] Levitin E., Kokodiy N. G., Timanjuk V. A., VedernikovaI. O., and Chan T. M. Measurements of the size an refractive index of $\mathrm{fe}_{3} \mathrm{O}_{4}$ nanoparticles. Inorganic Materials, 50:817-820, 2014.

[96] Ling Y., Yintang Z., Ran C., Danhua Z., Xiuhua W., Fang C., Jianxin W., and Maotian X. A highly sensitive resonance ligth scattering probe for alzheimer's amyloid-beta peptide based on $\mathrm{Fe}_{3} \mathrm{O}_{4} \mathrm{Au}$ composites. Talanta, 131:475-479, 2015.

[97] Mendoza L. J., Mu neton D., Schinca D. C., and Scaffardi L. B. Determination of plasma frequency, damping constant and size distribution from the complex dielectric function of noble metal nanoparticles. Journal of Applied Physics, 116:2331051-2331058, 2014.

[98] I. Robinson, L. D. Tung, S. Maenosono, C. Wealti, and N. T. K. Thanh. Synthesis of core-shell gold coated magnetic nanoparticles and their interaction with thiolated dna. Nanoscale, 2:2624-2630, 2010.

[99] Wang L., Luo J., Fan Q., Suzuki M., Suzuki H. S., Engelhard M. H., Lin Y., Kim N. Wang J. Q., and Zhong C. Monodispersed core-shell $\mathrm{Fe}_{3} \mathrm{O}_{4} \mathrm{Au}$ nanoparticles. The Journal of Physical Chemistry A, 109:21593-21601, 2005.

[100] Xi bin Xu, Zao Yi, Xi bo Li, Yu ying Wang, Xing Geng, Jiang shan Luo, Bing chi Luo, You gen Yi, and Yong jian Tang. Discrete dipole approximation simulation of the surface plasmon resonance of core/shell nanostructure and the study of resonance cavity effect. The Journal of Physical Chemistry C, 116:24046-24053, 2012.

[101] Wen-Hui Yang, George C. Schatz, and Richard P. Van Duyne. Discrete dipole approximation for calculating extinction and raman intensities for small particles with arbitrary shapes. The Journal of Chemical Physics, 103:869-875, 1995.

[102] Damon A. Smith and Kevin L. Stokes. Discrete dipole approximation for magnetooptical scattering calculations. Optics Express, 14:5746-5754, 2006. 
[103] B. T. Draine and P. J. Flatau. Discrete-dipole approximation for scattering calculations. Journal of the Optical Society of America A, 11:1491-1499, 1994.

[104] B. T. Draine and P. J. Flatau. Discrete-dipole approximation for periodic targets: theory and tests. Journal of the Optical Society of America A, 25:2693-2703, 2008.

[105] By P. V. Asharani, Swaminathan Sethu, Sajini Vadukumpully, Shaoping Zhong, Chwee Teck Lim, M. Prakash Hande, and Suresh Valiyaveettil. Investigations on the structural damage in human erythrocytes exposed to silver, gold, and platinum nanoparticles. Advanced Functional Materials, 20:1233-1242, 2010.

[106] Motonori Takamiya, Yusei Miyamoto, Toru Yamashita, Kentaro Deguchi, Yasuyuki Ohta, Yoshio Ikeda, Tohru Matsuura, and Koji Abe. Neurological and pathological improvements of cerebral infarction in mice with platinum nanoparticles. Journal of Neuroscience Research, 89:1125-1133, 2011.

[107] Yu Zhang, Ruo Yuan, Yaqin Chai, Jinfen Wang, and Huaan Zhong. Amperometric biosensor for nitrite and hydrogen peroxide based on hemoglobin immobilized on gold nanoparticles/polythionine/platinum nanoparticles modified glassy carbon electrode. Journal of Chemical Technology and Biotechnology, 87:570-574, 2011.

[108] Hitoshi Kato, Takashi Minami, Takaaki Kanazawa, and Yukichi Sasaki. Mesopores created by platinum nanoparticles in zeolite crystals. Angewandte Chemie, 116:1271-1271, 2004.

[109] Kun Zhao, Shuqi Zhuang, Zhu Chang, Haiyan Songm, Liming Dai, Pingang He, and Yuzhi Fang. Amperometric glucose biosensor based on platinum nanoparticles combined aligned carbon nanotubes electrode. Electroanalysis, 19:1069-1074, 2007.

[110] Alison Elder, Hong Yang, Roberto Gwiazda, Xiaowei Teng, Sally Thurston, Hua He, and Gunter Oberdorster. Testing nanomaterials of unknown toxicity: An example based on platinum nanoparticles of different shapes. Advanced Materials, 19:3124-3129, 2007. 
[111] J. L. Barr, R. L. Axelbaum, and M. E. Macias. Processing salt-encapsulated tantalum nanoparticles for high purity, ultra high surface area applications. Journal of Nanoparticle Research, 8:11-12, 2006.

[112] Fidel Martinez Gutierrez, Peggy L. Olive, Adriana Banuelos, Erasmo Orrantia, Nereyda Nino, Elpidio Morales Sanchez, Facundo Ruiz, Horacio Bach, and Yossef Av-Gay. Synthesis, characterization, and evaluation of antimicrobial and cytotoxic effect of silver and titanium nanoparticles. Nanomedicine: Nanotechnology, Biology, and Medicine, 6:681-688, 2010.

[113] W. DeLaCruz, C.Gallardo-Vega, S.Tougaard, and L.Cota. Growth mechanism of iron nanoparticles on $\left(\begin{array}{llll}0 & 0 & 0 & 1\end{array}\right)$ sapphire wafers. Microelectronics Journal, 39:1374-1375, 2008.

[114] Kato H., Minami T., Kanazawa T., and Sasaki Y. Mesopores created by platinum nanoparticles in zeolite crystals. Angew. Chem., 116:1271-1274, 2004.

[115] Ping-Ping Fang, Anny Jutand, Zhong-Qun Tian, and Christian Amatore. Au-Pd core-shell nanoparticles catalyze suzuki-miyaura reactions in water through pd leaching. Angew. Chem., 123:12392-12396, 2011.

[116] Maeda K, Teramura K, Lu D, Takata T, Saito N, Inoue Y, and Domen K. Photocatalyst releasing hydrogen from water. Nature, 16:7082, 2006.

[117] Aiguo Shen, Lifang Chen, Wei Xie, Juncheng Hu, Ao Zeng, Ryan Richards, and Jiming Hu. Triplex Auâagâc coreâshell nanoparticles as a novel raman label. Adv. Funct. Mater, 20:969-975, 2010.

[118] Jae-Woon Nah, Young-Il Jeong, and Chong-Su Cho. Drug-delivery system based on core-shell-type nanoparticles composed of poly( $\gamma$-benzyl-l-glutamate) and poly(ethylene-oxide). J. Appl. Polym. Sci., 75:1115-1126, 2000. 
[119] Diagnostic and Therapeutic Applications of Metal Nanoshells, in Nanofabrication Towards Biomedical Applications: Techniques, Tools, Applications, and Impact. Wiley-VCH Verlag GmbH Co. KGaA,, 2006.

[120] Viktoryia I. Shautsova, Viktor A. Zhuravkov, Olga V. Korolik, Andrei G. Novikau, Gvidona P. Shevchen, and koPeter I. Gaiduk. Effect of interparticle field enhancement in self-assembled silver aggregates on surface-enhanced raman scattering. Plasmonics, 9:993-999, 2014.

[121] Pengzhen Guo, Debabrata Sikdar, Xiqiang Huang, Kae Jye Si, Wei Xiong, Shu Gong, Lim Wei Yap, Malin Premaratned, and Wenlong Cheng. Plasmonic coreâshell nanoparticles for sers detection of the pesticide thiram: size- and shape-dependent raman enhancement. Nanoscale, 7:2862-2868, 2015.

[122] V. Mondes, E. Antonsson, J. Plenge, C. Raschpichler, I. Halfpap, A. Menski, C. Graf, M. F. Kling, and E. Ruhl. Plasmonic electric near-field enhancement in self-organized gold nanoparticles in macroscopic arrays. Appl. Phys. B: Lasers Opt., 122:155, 2016.

[123] Yu Zhang, Alejandro Manjavacas, Nathaniel J. Hogan, Linan Zhou, Ciceron Ayala-Orozco, Liangliang Dong, Jared K. Day, Peter Nordlander, and Naomi J. Halas. Toward surface plasmon-enhanced optical parametric amplification (spopa) with engineered nanoparticles: A nanoscale tunable infrared source. Nano Lett., 16:3373-3378, 2016.

[124] Mun Yeong Son, Young Jun Hong, and Yun Chan Kang. Superior electrochemical properties of $\mathrm{Co}_{3} \mathrm{O}_{4}$ yolk-shell powders with a filled core and multishells prepared by a one-pot spray pyrolysis. Chem. Commun., 49:5678, 2013.

[125] Chen Chen, Haiyan $\mathrm{Xu}$, Ling $\mathrm{Xu}$, Fengjun Zhang, Jinkuang Dong, and Hao Wang. One-pot synthesis of homogeneous coreâshell $\mathrm{Cu}_{2} \mathrm{O}$ films with nanoparticle-composed multishells and their photocatalytic properties. $R S C A d v$., 3:25010, 2013. 
[126] Ming Li, Scott K Cushing, Jianming Zhang, Jessica Lankford, Zoraida P Aguilar, Dongling Ma, and Nianqiang Wu. Shape-dependent surface-enhanced raman scattering in gold-raman-probe-silica sandwiched nanoparticles for biocompatible applications. Nanotechnology, 23(11):115501, 2012.

[127] Knight M. W., Liu L., Wang Y., Brown L., Mukherjee S., King N. S., EverittH O., Nordlander P., and Halas N. J. Aluminum plasmonic nanoantennas. Nano Letters, 12:6000-6004, 2012.

[128] Castro-Lopez M., Brinks D., Sapienza R., and van Hulst N. F. Aluminum for nonlinear plasmonics: Resonance-driven polarized luminescence of $\mathrm{Al}, \mathrm{Ag}$, and Au nanoantennas. Nano Letters, 21:4674-4678, 2011.

[129] N. P. Hylton, X. F. Li, V. Giannini, K. H. Lee, N. J. Ekins Daukes, J. Loo, D. Vercruysse, P. Van Dorpe, H. Sodabanlu, M. Sugiyama, and S. A. Maier. Loss mitigation in plasmonic solar cells: aluminium nanoparticles for broadband photocurrent enhancements in gaas photodiodes. Scientific Reports, 3:1-6, 2013.

[130] Ying Hu, Ryan C. Fleming, and Rebekah A. Drezek. Optical properties of gold-silica-gold multilayer nanoshells. Optics Express, 16:19579-19591, 2008.

[131] D.J. Wu and X.J. Liu. Tunable near-infrared optical properties of three-layered goldâsilicaâgold nanoparticles. Applied Physics B, 97:193-197, 2009.

[132] Jian Zhu, Ying Juan Ren, Shu Min Zhao, and Jun Wu Zhao. The effect of inserted gold nanosphere on the local field enhancement of gold nanoshell. Materials Chemistry and Physics, 133:1060- 1065, 2012.

[133] Jian Zhu, Ying juan Renb, Shu min Zhaob, and Jun wu Zhao. The effect of inserted gold nanosphere on the local field enhancement of gold nanoshell. Materials Chemistry and Physics, 133:1060-1065, 2012. 
[134] Jian Zhu, Jian Jun Li, and Jun Wu Zhao. The study of surface plasmon resonance in $\mathrm{Au}-\mathrm{Ag}-\mathrm{Au}$ three-layered bimetallic nanoshell: The effect of separate Ag layer. Plasmonics, 9:435-441, 2014.

[135] E. Prodan and P. Nordlander. Plasmon hybridization in spherical nanoparticles. The Journal of Chemical Physics, 120:5444-5454, 2004.

[136] E. Prodan and P. Nordlander. Electronic structure and polarizability of metallic nanoshells. Chemical Physics Letters, 352:140-146, 2002.

[137] Daniel W. Brandl, Chris Oubre, and Peter Nordlander. Plasmon hybridization in nanoshell dimers. The Journal of Chemical Physics, 123:024701-024701-6, 2005.

[138] Naveen Gandra and Srikanth Singamaneni. Bilayered raman-intense gold nanostructures with hidden tags (brights) for high-resolution bioimaging. Advanced Materials, 25:1022-1027, 2013.

[139] A. N. Shipway, E. Katz, and I. Willner. Nanoparticle arrays on surfaces for electronic, optical, and sensor applications. ChemPhysChem, 1:18-52, 2000.

[140] Jianping Ge, Yongxing Hu, and Yadong Yin. Highly tunable superparamagnetic colloidal photonic crystals. Angew. Chem., 119:7572 -7575, 2007.

[141] Valentina Morandi, Franco Marabelli, Vincenzo Amendola, Moreno Meneghetti, and Davide Comoretto. Colloidal photonic crystals doped with gold nanoparticles: Spectroscopy and optical switching properties. Adv. Funct. Mater., 17:2779-2786, 2007.

[142] Silvia Colodrero, Manuel Oca na, and Hernán Míguez. Nanoparticle-based one-dimensional photonic crystals. Langmuir, 24:4430-4434, 2008.

[143] Jianping Ge, Yongxing Hu, Tierui Zhang, Tuan Huynh, and Yadong Yin. Self-assembly and field-responsive optical diffractions of superparamagnetic colloids. Langmuir, 24:3671-3680, 2008. 
[144] Jianping Ge and Yadong Yin. Magnetically tunable colloidal photonic structures in alkanol solutions. Adv. Mater., 20:3485-3491, 2008.

[145] Md. Anamul Haque, Takayuki Kurokawa, Gen Kamita, Youfeng Yue, and Jian Ping Gong. Rapid and reversible tuning of structural color of a hydrogel over the entire visible spectrum by mechanical stimulation. Chem. Mater., 23:5200-5207, 2011.

[146] S. Ravi and A. Karthikeyan. Magnetically tunable superparamagnetic cobalt doped iron oxide colloidal nanocluster. Adv. Mat. Lett., 4:562-566, 2013.

[147] Fei Hu, Haiyang Lin, Zhaoshun Zhang, Fan Liao, Mingwang Shao, Yeshayahu Lifshitz, and Shuit-Tong Lee. Smart liquid sers substrates based on $\mathrm{fe}_{3} \mathrm{O}_{4} / \mathrm{Au}$ nanoparticles with reversibly tunable enhancement factor for practical quantitative detection. Scientific Reports, 4:7204, 2014.

[148] Yongxing $\mathrm{Hu}$, Le Hea, Xiaogang Han, Mingsheng Wang, and Yadong Yin. Magnetically responsive photonic films with high tunability and stability. Nano Research, 8(2):611-620, 2015.

[149] Eileen Armstrong and Colm O’Dwyer. Artificial opal photonic crystals and inverse opal structures â fundamentals and applications from optics to energy storage. $J$. Mater. Chem. C, 3:6109-6143, 2015.

[150] Tao Lu, Wenhong Peng, Shenmin Zhu, and Di Zhang. Bio-inspired fabrication of stimuliresponsive photonic crystals with hierarchical structures and their applications. Nanotechnology, 27:122001, 2016.

[151] Ilia L. Rasskazov, Sergei V. Karpov, and Vadim A. Markel. Nondecaying surface plasmon polaritons in linear chains of silver nanospheroids. Optics Letters, 38:4743-4746, 2013.

[152] Vadim A. Markel. Propagation of surface plasmons in ordered and disordered chains of metal nanospheres. Physical Review B, 75:085426-085426-10, 2007. 
[153] Vadim A. Markel, Vladimir M. Shalaev, and Evgeni Y. Poliakov. Fluctuations of light scattered by fractal clusters. Journal of the Optical Society of America A, 14:60-69, 1997.

[154] V. A. Markel. Coupled-dipole approach to scattering of light from a onedimensional periodic dipole structure. Journal of Modern Optics, 40:2281-2291, 1993.

[155] Yu-Rong Zhen, Kin Hung Fung, and C. T. Chan. Collective plasmonic modes of metal nano-particles in two-dimensional, periodic regular arrays. Physical Review B, 78:035419-035419-25, 2008.

[156] W. H. Weber and G. W. Ford. Propagation of optical excitations by dipolar interactions in metal nanoparticle chains. Physical Review B, 70:125429-125429-7, 2004.

[157] Britain Willingham and Stephan Link. Energy transport in metal nanoparticle chains via sub-radiant plasmon modes. Optics Express, 19:6450-6461, 2011.

[158] Andrea Alú and Nader Engheta. Guided propagation along quadrupolar chains of plasmonic nanoparticles. Physical Review B, 79:235412-235412-11, 2009.

[159] Mario N. Berberan and Manuel J. E. Prieto. Energy transfer in spherical geometry. Journal of the Chemical Society, Faraday Transactions 2: Molecular and Chemical Physics, 83:1391-1409, 1987.

[160] I. H. Malitson and M. J. Dodge. Refractive index and birefringence of synthetic sapphire. Journal of the Optical Society of America, 62:1336-1336, 1972.

[161] I. H. Malitson. Interspecimen comparison of the refractive index of fused silica. Journal of the Optical Society of America, 55:1205-1209, 1965.

[162] J. R. Devore. Refractive indices of rutile and sphalerite. Journal of the Optical Society of America, 41:416-419, 1951. 
[163] M. Daimon and A. Masumura. Measurement of the refractive index of distilled water from the near-infrared region to the ultraviolet region. Applied Optics, 46:3811-3820, 2007.

[164] George B. Arfken and Hans J. Weber. Mathematical Methods for Physicists. Elsevier, 7 edition, 2013.

[165] G. N. Watson. A Treatise on the Theory of Bessel Functions. Cambridge University Press, 2 edition, 1995.

[166] H. C. van de Hulst. Light Scattering by small particles. Dover publications, Inc, 1 edition, 1957.

[167] Bruce J. Berne and Robert Pecora. Dynamic light scattering with applications to chemistry, biology, and physics. john wiley \& sons, 2 edition, 2000.

[168] Y. Ekinci, H. H. Solak, and J. F. Loffler. Plasmon resonances of aluminum nanoparticles and nanorods. Journal of Applied Physics, 104:083107-083107-7, 2008.

[169] O. Merchiers, F. Moreno, F. González, J.M. Saiz, and G. Videen. Electromagnetic wave scattering from two interacting small spherical particles. influence of their optical constants, $\epsilon$ and $\mu$. Optics Communications, 269:1-7, 2007.

[170] Ying Hu, Timo A. Nieminen, Norman R. Heckenberg, and Halina Rubinsztein Dunlop. Antireflection coating for improved optical trapping. Journal of Applied Physics, 103:093119-093119-8, 2008.

[171] Landau L. D. and Lifshitz E. M. Electrodynamics of Continuous Media. Pergamon Press, 1 edition, 1960.

[172] Jorge Zuloaga, Emil Prodan, and Peter Nordlander. Quantum plasmonics: Optical properties and tunability of metallic nanorods. ACS Nano, 4:5269-5276, 2010. 
[173] Britain Willingham and Stephan Link. A kirchhoff solution to plasmon hybridization. Applied Physics B, 113:519-525, 2013.

[174] Larissa Wollet, Bettina Frank, Martin Schaferling, Martin Mesch, Sven Hein, and Harald Giessen. Plasmon hybridization in stacked metallic nanocups. Optical Materials Express, 2:1384-1390, 2012.

[175] H. Liu, D. A. Genov, D. M. Wu, Y. M. Liu, Z. W. Liu, C. Sun, S. N. Zhu, and X. Zhang. Magnetic plasmon hybridization and optical activity at optical frequencies in metallic nanostructures. Physical Review B, 76:073101-073101-3, 2007.

[176] Zhe Yuan and Shiwu Gao. Linear-response study of plasmon excitation in metallic thin films: Layer-dependent hybridization and dispersión. Physical Review B, 73:155411-155411-8, 2006.

[177] Afshin Moradi. Plasmon hybridization in metallic nanotubes with a nonconcentric core. Optics Communications, 282:3368-3370, 2009.

[178] Afshin Moradi. Plasmon hybridization in metallic nanotubes. Journal of Physics and Chemistry of Solids, 69:2936-2938, 2008.

[179] Afshin Moradi. Plasmon hybridization in tubular metallic nanostructures. Physica B, 405:2466-2469, 2010.

[180] Maj Frederiksen, Vladimir E. Bochenkov, Michael B. Cortie, and Duncan S. Sutherland. Plasmon hybridization and field confinement in multilayer metalâdielectric nanocups. The Journal of Physical Chemistry C, $117: 15782-15789,2013$.

[181] T. J. Davis, D. E. Gómez, and K. C. Vernon. Simple model for the hybridization of surface plasmon resonances in metallic nanoparticles. Nano Letters, 10:2618-2625, 2010. 
[182] Vikram Kulkarni, Emil Prodan, and Peter Nordlander. Quantum plasmonics: Optical properties of a nanomatryushka. Nano Letters, 13:5873-5879, 2014. 


\section{Agradecimientos}

A la Argentina por acogernos y por todo lo que nos brindo, al CONICET, por la beca y las ayudas. A la CIC por todas las ayudas. A la UNLP por todo y más. Al CIOp por ser otra casa para mi.

A mis directores por el tiempo y sobre todo la paciencia que tuvieron conmigo para tolerar todas mis grandes falencias. Esto se puede verificar porque el dropbox los delata (jajajaja) en sus horas de trabajo en casa a altas horas de la noche. Pero lo mas importante que les quiero agradecer es su amistad.

A los amigos incondicionales Adriana, Hernando, Fabiancillo y Carmen. A todos los investigadores del CIOp (Alberto, Faustito, Gustavo, Gabriel, Hector, Jessica, Jorge R., Jorge T., Lili, Marcelo, Mario Gall., Mario Gar., Miriam, Monica, Nestor, Pedro, Ricardo, Roberto.) de los cuales he aprendido muchas cosas (científicas y cotidianas sobre todo de Faustito muchas cotidianas). Se que se utilizan mas los apellidos que los nombres pero los colombianos somos así. Al personal de apoyo y administrativo del CIOp por sus colaboraciones (Adriana, Alberto, Alexis, Cecilia, Daniel, Edmundo, Eduardo, Gustavo, Ricardo, Sergio y Viviana.) en especial a Roxana que tanto molesto con los trabajos, a Matias con la compu.

A los compañeros de lucha los estudiantes de doctorado (Andrés, Alejandro, Angélica, Damian, Demian, Daniel, Luisa, Quique, Roberto y valentín (esta acá y no allá jajaja)) y en especial a David.

A mis hijos por el tiempo que me conceden para dedicar a la ciencia, a una de las mejores mujeres que pueden existir Dary por acompañarme incondicionalmente en 
cada uno de los proyectos que inicie sin muchas veces importar que no sean del todo coherentes.

A mis padres y Abuelas (es difícil describir, por no decir que imposible). Recuerdo una historia sobre un medico, cuyo âhobbyâ era plantar árboles en el enorme patio de su casa. Este medico hacia un enorme esfuerzo por plantar estos arboles todos los días. Pero un hecho particular acompañaba la planta de arboles; que jamás regaba sus arboles una vez los plantaba, lo que provocaba que sus arboles demoraran mucho en crecer.

El argumento del medico a esta practica era que, si regase sus plantas, las raíces se acomodarían en la superficie y quedarían siempre esperando por el agua fácil, que venía de encima.. Como él no las regaba, los árboles demorarían más para crecer, pero sus raíces tenderían a migrar hacia lo más profundo, en busca del agua y de los variados nutrientes encontrados en las capas más inferiores del suelo. Así, según él, los árboles tendrían raíces profundas y serían más resistentes a las intemperies.

Un día de un viento muy fuerte y helado, los árboles de la calle estaban arqueados, como si no estuviesen resistiendo al rigor del invierno, pero los arboles del medico estaban sólidos, prácticamente no se movían, resistiendo estoicamente aquel fuerte viento.

La naturaleza nos enseña muchas cosas si las sabemos ver. A esto solo añado una celebre frase "la felicidad no tiene que ser una finalidad sino la forma en la que vives". A. Einstein.

El ciclo de la vida enseña miles de cosas entre ellas que cada una de sus etapas tiene vivencias únicas e irrepetibles (por eso la mejor edad es la que cada uno tiene). En este sentido quiero manifestar el sentimiento que me mueve a realizar una de las cosas que me gusta la ciencia, pero esta pasión también conlleva a extraer tiempo que debe ser dedicado a los seres mas queridos en el mundo en mi caso a mis hijos. Este aparte de mi vida siempre me lo recuerdan los cuadros de Thomas Cole del viaje de la vida del año 1842 y a las cuales se les hace referencia en el libro Algún día este dolor te será útil de Peter Cameron. 


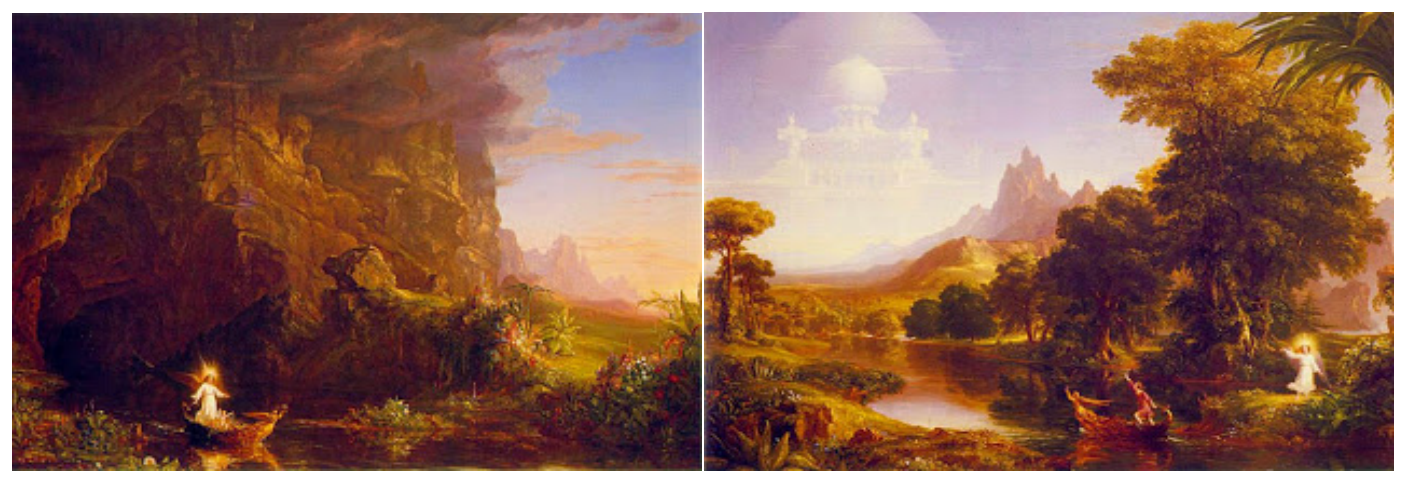

Thomas Cole, El Viaje de la Vida - La infancia- 1842

Thomas Cole, El Viaje de la Vida - La juventud- 1842

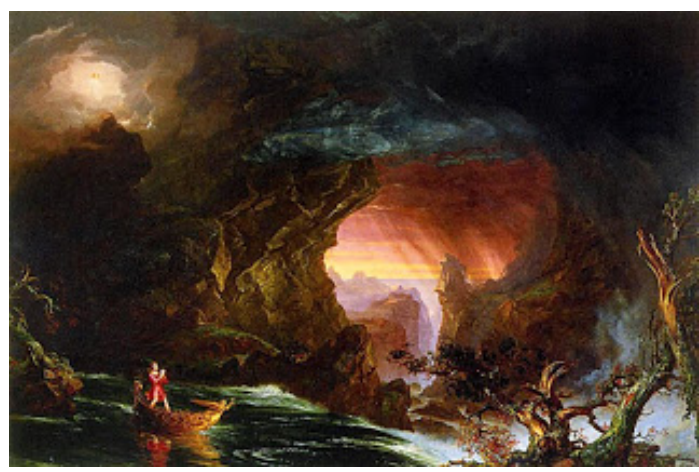

Thomas Cole, El Viaje de la Vida - La adultez- 1842

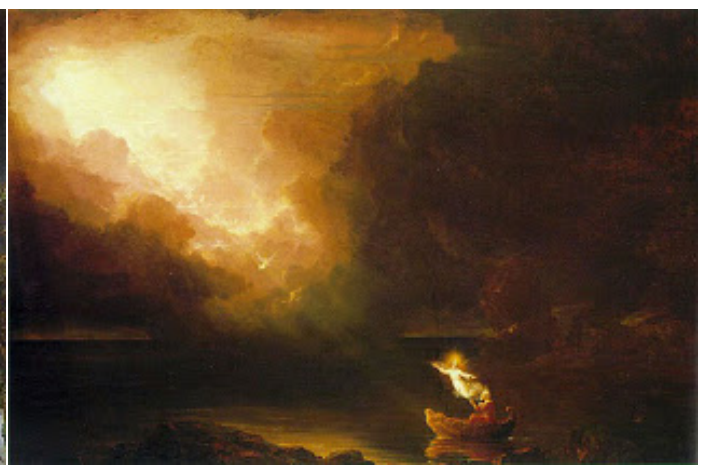

Thomas Cole, El Viaje de la Vida - La vejez- 1842 